International Journal of Bifurcation and Chaos, Vol. 11, No. 6 (2001) 1495-1591

(C) World Scientific Publishing Company

\title{
TESTING FOR LOCAL ACTIVITY AND EDGE OF CHAOS
}

\author{
TAO YANG and LEON O. CHUA \\ Electronics Research Laboratory and \\ Department of Electrical Engineering and Computer Sciences, \\ University of California at Berkeley, \\ Berkeley, California, CA 94720, USA
}

Received February 24, 2001; Revised April 18, 2001

\begin{abstract}
In this paper we study the local activity, local passivity and edge of chaos of continuous-time reaction-diffusion cellular nonlinear networks $(\mathrm{CNN})$ with one-port first-order, one-port secondorder, two-port second-order, two-port third-order and three-port third-order cells. We prove that the local passive regions determined by cell impedance $Z_{Q}(s)$ and cell admittance $Y_{Q}(s)$ for first- and second-order cells are equivalent to each other. We also present an efficient procedure to determine the edge-of-chaos parameter region by combining the local active regions derived from $Y_{Q}(s)$ and the pole locations of $Z_{Q}(s)$. In order to characterize the fundamental limitations of local passivity on the emergence of complexity we study the local active property from a parameter space spanned by both the cell parameters and the external excitations called the cell's port currents (in view of its interpretations from classical circuit theory). Analytical results of locally passive, restricted locally passive, edge-of-chaos and locally active parameter regions for $\mathrm{CNN}$ cells modeled by cubic nonlinearities are presented. We illustrate our results by analyzing CNN cells modeled by Chua's circuits with a cubic nonlinearity. We find that the morphology of the edge-of-chaos and the local active parameter regions have a close connection to the pattern formation behaviors of CNNs. Simulation results are presented to verify our theoretical results.
\end{abstract}

\section{Introduction}

The concept of local activity for reaction-diffusion cellular nonlinear networks (CNN) was first presented in [Chua, 1998]. The principles and applications of local activity and edge of chaos for different classes of continuous-time CNNs had been presented in [Min et al., 2000; Dogaru \& Chua, 1998c, 1998b, 1998a; Chua, 1999]. In this paper, we present new analytical results of locally passive and locally active parameter regions for different classes of CNNs. Since the basic concepts and terminologies of CNN had been presented in the book [Chua, 1998], we will only present some basic concepts of CNN to make this paper self-contained.

A general reaction-diffusion $\mathrm{CNN}$ made of " $m$-port" and nth-order cells $(m \leq n)$ is de- fined by

$\begin{aligned} \frac{d V_{1}(t)}{d t} & =f_{1}\left(V_{1}(t), V_{2}(t), \ldots, V_{n}(t)\right)+I_{1}(t) \\ \frac{d V_{2}(t)}{d t} & =f_{2}\left(V_{1}(t), V_{2}(t), \ldots, V_{n}(t)\right)+I_{2}(t) \\ & \vdots \\ \frac{d V_{m}(t)}{d t} & =f_{m}\left(V_{1}(t), V_{2}(t), \ldots, V_{n}(t)\right)+I_{m}(t) \\ \frac{d V_{m+1}(t)}{d t} & =f_{m+1}\left(V_{1}(t), V_{2}(t), \ldots, V_{n}(t)\right) \\ & \vdots \\ \frac{d V_{n}(t)}{d t} & =f_{n}\left(V_{1}(t), V_{2}(t), \ldots, V_{n}(t)\right) .\end{aligned}$


For any constant excitation $\mathbf{I}(t)=\left[\bar{I}_{1}, \bar{I}_{2}, \ldots \bar{I}_{m}\right], \bar{I}_{i} \in$ $\mathbb{R}$, the associated Local State Equation at a cell equilibrium point $Q \triangleq\left(\bar{V}_{1}, \bar{V}_{2}, \ldots, \bar{V}_{m}, \bar{I}_{1}, \bar{I}_{2}, \ldots, \bar{I}_{m}\right)$ obtained by solving Eq. (1) with $d V_{i}(t) / d t=0$, subject to zero initial state $v_{1}(0)=v_{2}(0)=\cdots=$ $v_{n}(0)=0$, is given by

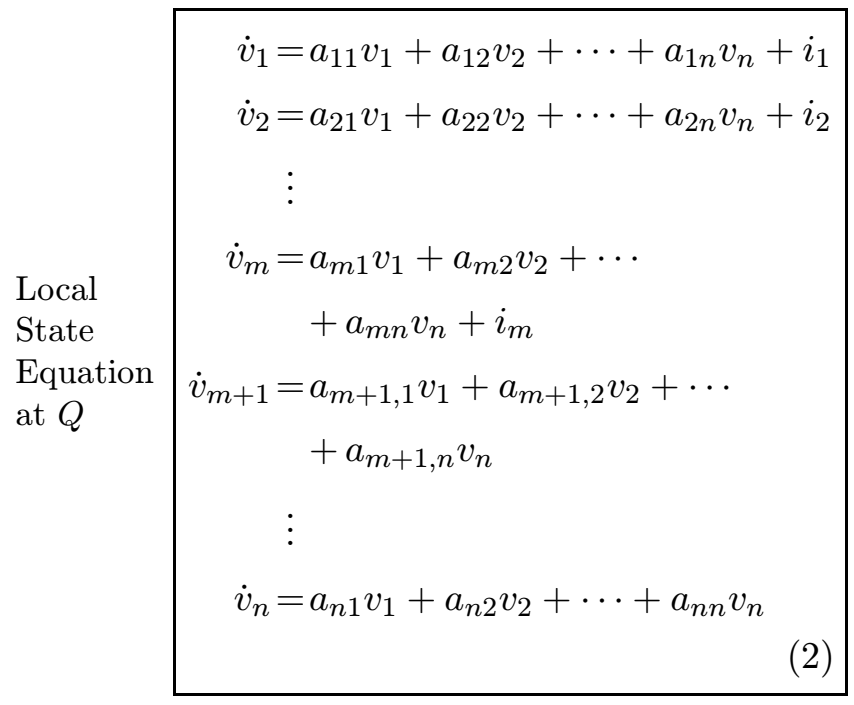

where

$$
\left.a_{i j} \triangleq \frac{\partial f_{i}\left(V_{1}, V_{2}, \ldots, V_{n}\right)}{\partial V_{j}}\right|_{Q}
$$

are the local cell coefficients at the cell equilibrium point $Q$. Using vector notations, we can recast Eq. (2) into

$$
\begin{aligned}
& \dot{\mathbf{v}}_{a}=\mathbf{A}_{a a} \mathbf{v}_{a}+\mathbf{A}_{a b} \mathbf{v}_{b}+\mathbf{i}_{a} \\
& \dot{\mathbf{v}}_{b}=\mathbf{A}_{b a} \mathbf{v}_{a}+\mathbf{A}_{b b} \mathbf{v}_{b}
\end{aligned}
$$

where

$$
\begin{aligned}
\mathbf{v}_{a} & =\left[v_{1}, v_{2}, \ldots, v_{m}\right]^{\top}, \\
\mathbf{i}_{a} & =\left[i_{1}, i_{2}, \ldots, i_{m}\right]^{\top}, \\
\mathbf{v}_{b} & =\left[v_{m+1}, v_{m+2}, \ldots, v_{n}\right]^{\top},
\end{aligned}
$$

and where $\mathbf{A}_{a a}, \mathbf{A}_{a b}, \mathbf{A}_{b a}$, and $\mathbf{A}_{b b}$ are appropriately partitioned submatrices.

\subsection{Local activity criteria for one-port reaction-diffusion CNN cells}

A one-port reaction-diffusion CNN cell is locally active at a cell equilibrium point $Q \triangleq\left(\bar{V}_{1}, \bar{I}_{1}\right)$ if, and only if, its cell impedance $Z_{Q}(s)$ at $Q$ satisfies at least one of the following four conditions:

A1. $Z_{Q}(s)$ has a pole in $\operatorname{Re}[s]>0$.

A2. $\operatorname{Re}\left[Z_{Q}(i \omega)\right]=1 / 2\left[Z_{Q}^{*}(i \omega)+Z_{Q}(i \omega)\right]<0$ for some $\omega=\omega_{0}$, where $\omega_{0}$ is any real number.

A3. $Z_{Q}(s)$ has a simple pole $s=i \omega_{p}$ on the imaginary axis, where its associated residue

$$
k_{-1} \triangleq \begin{cases}\lim _{s \rightarrow i \omega_{p}}\left(s-i \omega_{p}\right) Z_{Q}(s), & \text { if } \omega_{p}<\infty, \\ \lim _{\omega_{p} \rightarrow \infty} \frac{Z_{Q}\left(i \omega_{p}\right)}{i \omega_{p}}, & \text { if } \omega_{p}=\infty .\end{cases}
$$

is either a complex number, or a negative real number.

A4. $Z_{Q}(s)$ has a multiple pole on the imaginary axis.

\subsection{Local passivity criteria for one-port reaction-diffusion CNN cells}

A one-port Reaction-Diffusion CNN cell is locally passive at a cell equilibrium point $Q \triangleq\left(\bar{V}_{1}, \bar{I}_{1}\right)$ if, and only if, its cell impedance $Z_{Q}(s)$ at $Q$ satisfies the following conditions:

P1. $Z_{Q}(s)$ has no poles in $\operatorname{Re}[s]>0$.

P2. $\operatorname{Re}\left[Z_{Q}(i \omega)\right]=1 / 2\left[Z_{Q}^{*}(i \omega)+Z_{Q}(i \omega)\right] \geq 0$ for all real $\omega$ where $s=i \omega$ is not a pole of $Z_{Q}(s)$.

P3. If $s=s_{p}$ is a pole of $Z(s)$ on the imaginary axis, i.e. $s_{p}=i \omega_{p}$, then $s_{p}$ must be a simple pole and its residue must be a positive real number.

\subsection{Local activity criteria for two-port reaction-diffusion CNN cells}

A two-port Reaction-Diffusion CNN cell is locally active at a cell equilibrium point $Q \triangleq$ $\left(\bar{V}_{1}, \bar{V}_{2}, \bar{I}_{1}, \bar{I}_{2}\right)$ if, and only if, its cell impedance matrix $\mathbf{Z}_{Q}(s)$ at $Q$ satisfies at least one of the following four conditions:

A1. $\mathbf{Z}_{Q}(s)$ has a pole in $\operatorname{Re}[s]>0$.

A2. $\mathbf{Z}_{Q}^{H}(i \omega) \triangleq \mathbf{Z}_{Q}^{\dagger}(i \omega)+\mathbf{Z}_{Q}(i \omega)$ is not a positive semi-definite matrix at some $\omega=\omega_{0}$, where $\omega_{0}$ is any real number; i.e. there exist some $\omega=\omega_{0}$ and some constant (real or complex) vector $\hat{\mathbf{i}}=\left[\hat{i}_{1}, \hat{i}_{2}\right]$ such that the real number ${ }^{1}$

$$
\hat{\mathbf{i}}^{\dagger} \mathbf{Z}_{Q}^{H}(i \omega) \hat{\mathbf{i}}<0
$$

\footnotetext{
${ }^{1}$ We use the superscripts " $\mathrm{H}$ " and "†" to denote the "Hermitian" and "transpose" operators, respectively.
} 
A3. $\mathbf{Z}_{Q}(s)$ has a simple pole $s=i \omega_{p}$ on the imaginary axis where its associated residue matrix

$$
\mathbf{K}_{-1} \triangleq \begin{cases}\lim _{s \rightarrow i \omega_{p}}\left(s-i \omega_{p}\right) \mathbf{Z}_{Q}(s), & \text { if } \omega_{p}<\infty \\ \lim _{\omega_{p} \rightarrow \infty} \frac{\mathbf{Z}\left(i \omega_{p}\right)}{i \omega_{p}}, & \text { if } \omega_{p}=\infty\end{cases}
$$

is either not a Hermitian matrix, or else not a positive semi-definite matrix.

A4. $\mathbf{Z}_{Q}(s)$ has a multiple pole on the imaginary axis.

\subsection{Local passivity criteria for two-port reaction-diffusion CNN cells}

A two-port Reaction-Diffusion CNN cell is locally passive at a cell equilibrium point $Q \triangleq$ $\left(\bar{V}_{1}, \bar{V}_{2}, \bar{I}_{1}, \bar{I}_{2}\right)$ if, and only if, its cell impedance matrix $\mathbf{Z}_{Q}(s)$ at $Q$ satisfies the following conditions:

P1. $\mathbf{Z}_{Q}(s)$ has no pole in $\operatorname{Re}[s]>0$.

P2. $\quad \mathbf{Z}_{Q}^{H}(i \omega) \triangleq \mathbf{Z}_{Q}^{\dagger}(i \omega)+\mathbf{Z}_{Q}(i \omega)$ is positive semidefinite for all real $\omega$ where $s=i \omega$ is not a pole of $\mathbf{Z}_{Q}(s)$.

P3. If $s=s_{p}$ is a pole of $\mathbf{Z}_{Q}(s)$ on the imaginary axis, i.e. $s=i \omega_{p}$, then it must be a simple pole and its associated residue matrix

$$
\mathbf{K}_{-1} \triangleq \begin{cases}\lim _{s \rightarrow i \omega_{p}}\left(s-i \omega_{p}\right) \mathbf{Z}_{Q}(s), & \text { if } \omega_{p}<\infty \\ \lim _{\omega_{p} \rightarrow \infty} \frac{\mathbf{Z}\left(i \omega_{p}\right)}{i \omega_{p}}, & \text { if } \omega_{p}=\infty\end{cases}
$$

is a positive semi-definite Hermitian matrix.

\subsection{Remarks}

Since the local state equation at $Q$ in Eq. (2) depends on the cell equilibrium point $Q=$ $\left(\bar{V}_{1}, \bar{V}_{2}, \ldots, \bar{V}_{m}, \bar{I}_{1}, \bar{I}_{2}, \ldots, \bar{I}_{m}\right)$ where the constants $\bar{I}_{1} \in \mathbb{R}, \bar{I}_{2} \in \mathbb{R}, \ldots, \bar{I}_{m} \in \mathbb{R}$ are independent parameters, ${ }^{2}$ we need to distinguish between the following two terminologies:

1. Local passive cells must be locally passive at every cell equilibrium points with arbitrary $\bar{I}_{1} \in \mathbb{R}$, $\bar{I}_{2} \in \mathbb{R}, \ldots, \bar{I}_{m} \in \mathbb{R}$.

2. Restricted local passive cells need only be locally passive at all cell equilibrium points with some proper subset of $\bar{I}_{1} \in \mathbb{R}, \bar{I}_{2} \in \mathbb{R}, \ldots, \bar{I}_{m} \in \mathbb{R}$.
Remark. It follows from classical circuit theory that to test local passivity or local activity, we can substitute the cell impedance $Z_{Q}(s)$ by the cell admittance $Y_{Q}(s) \triangleq 1 / Z_{Q}(s)$ in the above test criteria.

In this paper we use extensively the basic results on Hermitian matrices summarized in the following two theorems [Franklin, 1968].

Theorem 1. A Hermitian matrix is positive definite if, and only if, all its eigenvalues are positive.

Theorem 2. Let $H=\left(h_{i j}\right)_{n \times n}$ be an $n \times n$ Hermitian matrix. Then $H$ is positive definite if, and only if, all of its determinants are positive; namely,

$h_{11}>0,\left|\begin{array}{cc}h_{11} & h_{12} \\ h_{21} & h_{22}\end{array}\right|>0, \ldots,\left|\begin{array}{ccc}h_{11} & \cdots & h_{1 n} \\ \vdots & \cdots & \vdots \\ h_{n 1} & \cdots & h_{n n}\end{array}\right|>0$.

\section{One-Port CNN Cells}

In this section we study CNNs made of interconnections of one-port cells. In particular, we will study one-port first-order cells and one-port second-order cells. We will show that the local passive results derived from $Z_{Q}(s)$ and $Y_{Q}(s)$ are equivalent to each other for one-port cells studied in this section.

\subsection{One-port first-order CNN cells}

Let us study first the local passive regions of oneport first-order cells by using $Z_{Q}(s)$ and $Y_{Q}(s)$.

Results from $Z_{Q}(s)$

For one-port first-order cells, the linearized cell state equation at an equilibrium point $Q$ is given by

$$
\dot{v}_{1}=a_{11} v_{1}+i_{1}
$$

from which we derive the cell impedance

$$
Z_{Q}(s)=\frac{1}{s-a_{11}} \text {. }
$$

$\mathrm{P} 1\left(Z_{Q}(s)\right)$. Conditions for " $\operatorname{Re}\left[s_{p}\right] \leq 0$ ".

\footnotetext{
${ }^{2}$ In terms of classic electronic circuit theory, these constants are called "dc biases", and correspond to the dc power supply essential to establish a $d c$ operating point $Q$.
} 
The parameter regions satisfying $\mathrm{P} 1$ are given by

$$
a_{11} \leq 0 .
$$

$\mathrm{P} 2\left(Z_{Q}(s)\right)$. Conditions for " $\operatorname{Re}[Z(i \omega)] \geq 0$, for all $\omega \in \mathbb{R}$ and $s=i \omega$ is not a pole of $Z(s)$ ".

The parameter regions are given as follows.

$$
Z(i \omega)=\frac{1}{i \omega-a_{11}}=\frac{-i \omega-a_{11}}{\omega^{2}+a_{11}^{2}} .
$$

Therefore from $\operatorname{Re}[Z(i \omega)] \geq 0$ we have

$$
a_{11} \leq 0 .
$$

When $a_{11}=0, s=0$ is a pole of $Z(s)$, therefore the parameter region satisfying $\mathrm{P} 2$ is given by

$$
a_{11}<0
$$

which is a subset of $\mathrm{P} 1$. This means that the edge-of-chaos region can only possibly be located at $a_{11}=0$. However, our next condition shows that the parameter point $a_{11}=0$ satisfies P3. Therefore, the edge-of-chaos region is empty for one-port first-order cells.

$\mathrm{P} 3\left(Z_{Q}(s)\right)$. Conditions for " $s_{p}=i \omega$ is a simple pole and has positive residue."

Only when $a_{11}=0$ does $Z(s)$ have a simple pole on the imaginary axis. In this case, the associated residue is $K_{-1}=1>0$. It follows that P3 is satisfied when

$$
a_{11}=0 .
$$

Therefore, the local passive parameter regions are given by $\left\{\left[\begin{array}{lll}\mathrm{P} 1 & \mathbb{A N D} & \mathrm{P} 2\end{array}\right] \quad \mathbb{O R} \quad \mathrm{P} 3\right\}$; namely,

$$
a_{11} \leq 0 \text {. }
$$

If follows that for one-port first-order cells, the locally active regions are located at

$$
a_{11}>0 .
$$

There is no edge of chaos region.

\section{Results from $Y_{Q}(s)$}

The cell admittance is given by

$$
Y_{Q}(s)=Z_{Q}^{-1}(s)=s-a_{11} .
$$

$\mathrm{P} 1\left(Y_{Q}(s)\right)$. Conditions for " $\operatorname{Re}\left[s_{p}\right] \leq 0$ ".

Since there is only one pole; namely, at infinity, the residue is given by

$$
\lim _{\omega \rightarrow \infty} \frac{Y_{Q}(i \omega)}{i \omega}=1
$$

This means that this criterion is satisfied for all parameters.

$\mathrm{P} 2\left(Y_{Q}(s)\right)$. Conditions for " $\operatorname{Re}[Y(i \omega)] \geq 0$, for all $\omega \in \mathbb{R}$ and $s=i \omega$ is not a pole of $Y(s)$."

The parameter regions are given as follows.

$$
Y(i \omega)=i \omega-a_{11} \text {. }
$$

Therefore from $\operatorname{Re}[Y(i \omega)] \geq 0$ we have

$$
a_{11} \leq 0 \text {. }
$$

$\mathrm{P} 3\left(Y_{Q}(s)\right)$. Conditions for " $s_{p}=i \omega$ is a simple pole and has positive residue."

In this case, the residue is given by

$$
K_{-1}=\lim _{\omega \rightarrow \infty} \frac{i \omega-a_{11}}{i \omega}=1>0 .
$$

Therefore, this condition is satisfied at the pole at infinity. In this case, it contributes nothing to the passive parameter region.

Therefore, the local passive parameter regions are given by $\left\{\left[\begin{array}{lll}\mathrm{P} 1 & \mathbb{A N D} & \mathrm{P} 2\end{array}\right] \quad \mathbb{O} \mathbb{R} \quad \mathrm{P} 3\right\}$; namely,

$$
a_{11} \leq 0 \text {. }
$$

From above we can see that the conclusions from $Z_{Q}(s)$ and $Y_{Q}(s)$ for one-port first-order cells are identical, as expected.

\subsection{One-port second-order CNN cells}

Many well-known reaction-diffusion CNNs have only one nonzero diffusion coefficient and two state variables. The linearized cell state equation at an equilibrium point $Q$ is given by

$$
\begin{aligned}
& \dot{v}_{1}=a_{11} v_{1}+a_{12} v_{2}+i_{1}, \\
& \dot{v}_{2}=a_{21} v_{1}+a_{22} v_{2}
\end{aligned}
$$

which is a one-port second-order cell.

The $C N N$ cell impedance $Z_{Q}(s)$ associated with Eq. (23) is given by

$$
Z_{Q}(s)=\frac{\left(s-a_{22}\right)}{s^{2}-T s+\Delta} .
$$

where

$$
T \triangleq a_{11}+a_{22}, \quad \Delta \triangleq a_{11} a_{22}-a_{12} a_{21}
$$

are the trace and determinant, respectively, of the associated Jacobian matrix

$$
\mathbf{J}_{Q}=\left[\begin{array}{ll}
a_{11} & a_{12} \\
a_{21} & a_{22}
\end{array}\right]
$$

evaluated at the cell equilibrium point $Q$. 


\subsubsection{Passivity of one-port second-order CNN Cells}

Let us study next the local passivity of one-port second-order cells based on $Z_{Q}(s)$ and $Y_{Q}(s)$.

\section{Conclusions from $Z_{Q}(s)$}

Let us find the parameter regions where the properties $\mathrm{P} 1$ to $\mathrm{P} 3$ are satisfied with respect to $Z_{Q}(s)$.

$\mathrm{P} 1\left(Z_{Q}(s)\right)$. Conditions for "Re$\left[s_{p}\right] \leq 0$."

The parameter regions are given by

$$
T \leq 0 \quad \mathbb{A N D} \quad \Delta \geq 0 .
$$

$\mathrm{P} 2\left(Z_{Q}(s)\right)$. Conditions for "Re$\left[Z_{Q}(i \omega)\right] \geq 0$ for all $\omega \in \mathbb{R}$ and $s=i \omega$ is not a pole of $Z(s)$."

The parameter regions are given as follows:

$$
Z_{Q}(i \omega)=\frac{i \omega-a_{22}}{-\omega^{2}-i T \omega+\Delta}
$$

It follows from $\operatorname{Re}\left[Z_{Q}(i \omega)\right] \geq 0$ that

$$
-a_{22} \Delta-a_{11} \omega^{2} \geq 0
$$

from which we obtain, for very large or very small $\omega$

$$
a_{11} \leq 0 \quad \mathbb{A N D} \quad a_{22} \Delta \leq 0
$$

which is equivalent to

$$
T \leq a_{22} \quad \mathbb{A N D} \quad a_{22} \Delta \leq 0 .
$$

$\mathrm{P} 3\left(Z_{Q}(s)\right)$. Conditions for " $Z_{Q}(s)$ has a simple pole at $s_{p}=i \omega$ with real and positive residue."

The parameter regions are given by

$$
a_{11}=0 \quad \mathbb{A N D} \quad a_{22}=0 \quad \mathbb{A N D} \quad a_{12} a_{21}<0 .
$$

\section{Conclusions from $Y_{Q}(s)$}

Let us find the parameter regions where the properties $\mathrm{P} 1$ to $\mathrm{P} 3$ are satisfied with respect to $Y_{Q}(s)$ which is given by

$$
Y_{Q}(s)=\frac{s^{2}-T s+\Delta}{s-a_{22}} .
$$

$\mathrm{P} 1\left(Y_{Q}(s)\right)$. Conditions for "Re $\left[s_{p}\right] \leq 0$."

The parameter regions are given by

$$
a_{22} \leq 0 \text {. }
$$

$\mathrm{P} 2\left(Y_{Q}(s)\right)$. Conditions for " $\operatorname{Re}[Y(i \omega)] \geq 0$ for all $\omega \in \mathbb{R}$ and $s=i \omega$ is not a pole of $Y_{Q}(s)$."

The parameter regions must satisfy

$$
\begin{aligned}
\operatorname{Re}\left[Y_{Q}(i \omega)\right] & =\operatorname{Re}\left[\frac{-\omega^{2}-i T \omega+\Delta}{i \omega-a_{22}}\right] \\
& =\frac{a_{22} \omega^{2}-a_{22} \Delta-T \omega^{2}}{\omega^{2}+a_{22}^{2}} \\
& \geq 0, \quad \forall \omega \in \mathbb{R}
\end{aligned}
$$

from which we have

$$
a_{22} \omega^{2}-a_{22} \Delta-T \omega^{2} \geq 0, \quad \forall \omega \in \mathbb{R} .
$$

It follows that

$$
a_{22} \Delta \leq 0 \quad \mathbb{A N D} \quad T \leq a_{22} .
$$

P3 $\left(Y_{Q}(s)\right)$. Conditions for " $Y_{Q}(s)$ has a simple pole at $s_{p}=i \omega$ with real and positive residue."

In this case $a_{22}=0$ must be satisfied and the parameter regions are given by

$$
K_{-1}=\Delta>0
$$

which leads to

$$
a_{22}=0 \quad \mathbb{A N D} \quad \Delta>0 .
$$

\section{Remarks}

Let us now prove that the local passive regions derived from $Z_{Q}(s)$ and $Y_{Q}(s)$ are identical. Denote the conditions derived with respect to $Y_{Q}(s)$ from $\mathrm{P} 1, \mathrm{P} 2$ and $\mathrm{P} 3$, as $\mathrm{P} 1\left(Y_{Q}(s)\right), \mathrm{P} 2\left(Y_{Q}(s)\right)$ and $\mathrm{P} 3\left(Y_{Q}(s)\right)$, and denote the conditions derived with respect to $Z_{Q}(s)$ from $\mathrm{P} 1, \mathrm{P} 2$ and $\mathrm{P} 3$, as $\mathrm{P} 1\left(Z_{Q}(s)\right)$, $\mathrm{P} 2\left(Z_{Q}(s)\right)$ and $\mathrm{P} 3\left(Z_{Q}(s)\right)$, respectively.

Theorem $3 \quad\left[\mathrm{P} 1\left(Y_{Q}(s)\right) \quad \mathbb{A N D} \quad \mathrm{P} 2\left(Y_{Q}(s)\right)\right] \quad \Leftrightarrow$ $\left[\mathrm{P} 1\left(Z_{Q}(s)\right) \quad \mathbb{A N D} \quad \mathrm{P} 2\left(Z_{Q}(s)\right)\right]$.

Proof

1. $\left[\mathrm{P} 1\left(Y_{Q}(s)\right) \quad \mathbb{A N D} \quad \mathrm{P} 2\left(Y_{Q}(s)\right)\right] \Leftarrow\left[\mathrm{P} 1\left(Z_{Q}(s)\right)\right.$ $\left.\mathbb{A N D} \mathrm{P} 2\left(Z_{Q}(s)\right)\right]:\left[\begin{array}{llll}\mathrm{P} 1\left(Z_{Q}(s)\right) & \mathbb{A N D} & \mathrm{P} 2\left(Z_{Q}(s)\right)\end{array}\right]$ is given by

$$
\begin{aligned}
& T \leq 0 \quad \mathbb{A N D} \quad \Delta \geq 0 \quad \mathbb{A N D} \\
& T \leq a_{22} \quad \mathbb{A N D} \quad a_{22} \Delta \leq 0 .
\end{aligned}
$$


From $\Delta \geq 0$ and $a_{22} \Delta \leq 0$ we have $a_{22} \leq 0$, and Eq. (40) becomes

$$
T \leq 0 \quad \mathbb{A N D} \quad a_{22} \leq 0 \quad \mathbb{A N D} \quad T \leq a_{22} \quad \mathbb{A N D} \quad a_{22} \Delta \leq 0 .
$$

Since $T \leq a_{22}$ and $a_{22} \leq 0$ we know $T \leq 0$; therefore Eq. (41) becomes

$$
a_{22} \leq 0 \quad \mathbb{A N D} \quad T \leq a_{22} \quad \mathbb{A N D} \quad a_{22} \Delta \leq 0
$$

which is exactly the conditions given by $\left[\mathrm{P} 1\left(Y_{Q}(s)\right) \quad \mathbb{A N D} \quad \mathrm{P} 2\left(Y_{Q}(s)\right)\right]$.

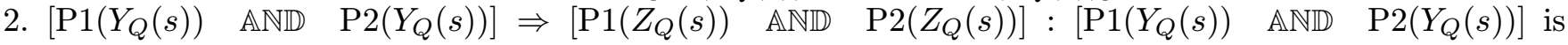
given by

$$
a_{22} \leq 0 \quad \mathbb{A N D} \quad T \leq a_{22} \quad \mathbb{A N D} \quad a_{22} \Delta \leq 0 .
$$

Since $a_{22} \leq 0$ and $T \leq a_{22}$, we know $T \leq 0$, and Eq. (43) is equivalent to

$$
T \leq 0 \quad \mathbb{A N D} \quad a_{22} \leq 0 \quad \mathbb{A N D} \quad T \leq a_{22} \quad \mathbb{A N D} \quad a_{22} \Delta \leq 0 .
$$

Since $a_{22} \leq 0$ and $a_{22} \Delta \leq 0$, we have $\Delta \geq 0$. It follows that Eq. (44) is equivalent to

$$
T \leq 0 \quad \mathbb{A N D} \quad \Delta \geq 0 \quad \mathbb{A N D} \quad T \leq a_{22} \quad \mathbb{A N D} \quad a_{22} \Delta \leq 0
$$

which is exactly the the conditions given by $\left[\mathrm{P} 1\left(Z_{Q}(s)\right) \quad \mathbb{A N D} \quad \mathrm{P} 2\left(Z_{Q}(s)\right)\right]$.

Summarizing the above results, we have

$$
\left[\mathrm{P} 1\left(Y_{Q}(s)\right) \quad \mathbb{A N D} \quad \mathrm{P} 2\left(Y_{Q}(s)\right)\right] \Leftrightarrow\left[\mathrm{P} 1\left(Z_{Q}(s)\right) \quad \mathbb{A N D} \quad \mathrm{P} 2\left(Z_{Q}(s)\right)\right] .
$$

Remark. This theorem shows that the local passive parameter regions derived from $Y_{Q}(s)$ and $Z_{Q}(s)$ are identical. Furthermore, since the local active regions and local passive regions are complementary sets it follows that the local active regions derived from $Y_{Q}(s)$ and $Z_{Q}(s)$ are also identical. Can we go further and argue that the edge-of-chaos regions derived from $Y_{Q}(s)$ and $Z_{Q}(s)$ are also identical? The answer is $N O$, as demonstrated in the counter example given in the next section.

\subsubsection{Edge-of-chaos parameter regions for one-port second-order CNN cells}

The edge-of-chaos region [Chua, 1998] is defined by $\mathrm{P} 1\left(Z_{Q}(s)\right) \quad \mathbb{A N D} \quad \mathbb{N O T}\left[\mathrm{P} 2\left(Z_{Q}(s)\right)\right]$. Here we show for this example that

$\mathrm{P} 1\left(Z_{Q}(s)\right) \quad \mathbb{A N D} \quad \mathbb{N O T}\left[\mathrm{P} 2\left(Z_{Q}(s)\right)\right]$ and

$\mathrm{P} 1\left(Y_{Q}(s)\right) \quad \mathbb{A N D} \quad \mathbb{N O T}\left[\mathrm{P} 2\left(Y_{Q}(s)\right)\right]$ are not equivalent.

$\mathrm{P} 1\left(Z_{Q}(s)\right) \quad \mathbb{A N D} \quad \mathbb{N O T}\left[\mathrm{P} 2\left(Z_{Q}(s)\right)\right]$ is given by

$$
\left[\begin{array}{lll}
T<0 & \mathbb{A N D} & \Delta>0
\end{array} \quad \mathbb{A N D} \quad\left[\begin{array}{llll}
T>a_{22} & \mathbb{O} R & a_{22} \Delta>0
\end{array}\right]\right.
$$

and $\mathrm{P} 1\left(Y_{Q}(s)\right) \quad \mathbb{A N D} \quad \mathbb{N O T}\left[\mathrm{P} 2\left(Y_{Q}(s)\right)\right]$ is given by

$$
\left[a_{22}<0\right] \quad \mathbb{A N D} \quad\left[\begin{array}{lll}
T>a_{22} & \mathbb{O} \mathbb{R} & a_{22} \Delta>0
\end{array}\right]
$$

1. From Eq. (46) we have

$$
\begin{aligned}
& {\left[\begin{array}{lllllll}
a_{11}+a_{22}<0 & \mathbb{A N D} & \Delta>0 & \mathbb{A N D} & a_{11}+a_{22}>a_{22}
\end{array}\right] \quad \mathbb{O R} \quad\left[\begin{array}{lll}
a_{11}+a_{22}<0 & 0
\end{array}\right.} \\
& \left.\mathbb{A N D} \quad \Delta>0 \quad \mathbb{A N D} \quad a_{22} \Delta>0\right]
\end{aligned}
$$

Equation (48) implies

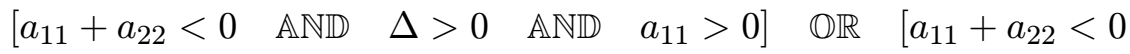

$$
\begin{aligned}
& \left.\mathbb{A N D} \quad \Delta>0 \quad \mathbb{A N D} \quad a_{22}>0\right]
\end{aligned}
$$


and

$$
\begin{aligned}
& {\left[\begin{array}{lllllll}
a_{11}+a_{22}<0 & \mathbb{A N D} & \Delta>0 & \mathbb{A N D} & a_{11}>0
\end{array}\right] \quad \mathbb{O} \mathbb{R} \quad\left[a_{11}+a_{22}<0\right.} \\
& \left.\mathbb{A N D} \quad \Delta>0 \quad \mathbb{A N D} \quad a_{22} \Delta>0\right] \\
& {\left[\begin{array}{lllllll}
a_{22} \leq 0 & \mathbb{A N D} & a_{11}+a_{22}<0 & \mathbb{A N D} & \Delta>0 & \mathbb{A N D} & a_{11}>0
\end{array}\right] \quad \mathbb{O} \mathbb{R}} \\
& {\left[\begin{array}{lllll}
a_{22}>0 & \mathbb{A N D} & a_{11}+a_{22}<0 & \mathbb{A N D} & \Delta>0
\end{array}\right]}
\end{aligned}
$$

2. From Eq. (47) we have

$$
\left[a_{22}<0\right] \quad \mathbb{A N D} \quad\left[a_{11}+a_{22}>a_{22} \quad \mathbb{O} \mathbb{R} \quad a_{22} \Delta>0\right]
$$

Equation (52) imples

$$
\left[\begin{array}{lll}
a_{22}<0 & \mathbb{A N D} & a_{11}>0
\end{array}\right] \quad \mathbb{O} \mathbb{R} \quad\left[\begin{array}{llll}
a_{22}<0 & \mathbb{A N D} & a_{22} \Delta>0
\end{array}\right]
$$

Hence,

$$
\left[\begin{array}{llllll}
a_{22}<0 & \mathbb{A N D} & a_{11}>0
\end{array}\right] \quad \mathbb{O} \mathbb{R} \quad\left[\begin{array}{lll}
a_{22}<0 & \mathbb{A N D} & \Delta<0
\end{array}\right]
$$

We conclude that the two set of conditions in Eqs. (51) and (54) are not equivalent. Therefore, the edge-of-chaos regions cannot be found from the conditions derived from $Y_{Q}(s)$. Based on the original definition in [Chua, 1998], to find edge-of-chaos region it is necessary to find the poles of $Z_{Q}(s)$; namely,

Edge-of-chaos region $=($ local active region $) \mathbb{A N D}$ (region where poles of $Z_{Q}(s)$ are on the left-half $s$-plane).

\subsubsection{Active parameter regions for one-port second-order CNN cells}

Let us find next the parameter regions where the properties $\mathrm{A} 1\left(Z_{Q}(s)\right)$ to $\mathrm{A} 4\left(Z_{Q}(s)\right)$ are satisfied.

$\mathrm{A} 1\left(Z_{Q}(s)\right)$. Conditions for "Re$\left[s_{p}\right]>0$ " are those for "NOT (P1)" and are given by

$$
T>0 \quad \mathbb{O} \mathbb{R} \quad \Delta<0 .
$$

$\mathrm{A} 2\left(Z_{Q}(s)\right)$. Conditions for "NOT $(\mathrm{P} 2)$ " are given by

$$
a_{11}>0 \quad \mathbb{O R} \quad a_{22} \Delta>0 .
$$

$\mathrm{A} 3\left(Z_{Q}(s)\right)$. Conditions for " $Z(s)$ has a simple pole at $s_{p}= \pm i \omega$ with complex or negative residue" are given by

$$
T=0 \quad \mathbb{A N D} \quad \Delta>0 \quad \mathbb{A N D} \quad a_{22} \neq 0 .
$$

$\mathrm{A} 4\left(Z_{Q}(s)\right)$. Conditions for " $Z(s)$ has a multiple

\section{pole" are given by}

$$
T=0 \quad \mathbb{A N D} \quad \Delta=0 \quad \mathbb{A N D} \quad a_{22} \neq 0 .
$$

\section{Two-Port and Second-Order CNN Cells}

The linearized cell state equation at an equilibrium point $Q$ is given by

$$
\begin{aligned}
& \dot{v}_{1}=a_{11} v_{1}+a_{12} v_{2}+i_{1}, \\
& \dot{v}_{2}=a_{21} v_{1}+a_{22} v_{2}+i_{2} .
\end{aligned}
$$

The cell impedance at an equilibrium point $Q$ is given by

$$
\begin{aligned}
& \mathbf{Z}_{Q}(s)=\left[\begin{array}{cc}
s-a_{11} & -a_{12} \\
-a_{21} & s-a_{22}
\end{array}\right]^{-1} \\
& =\frac{1}{\left(s-a_{11}\right)\left(s-a_{22}\right)-a_{12} a_{21}} \\
& \times\left[\begin{array}{cc}
s-a_{22} & a_{12} \\
a_{21} & s-a_{11}
\end{array}\right] \\
& =\frac{1}{s^{2}-\left(a_{11}+a_{22}\right) s+\left(a_{11} a_{22}-a_{12} a_{21}\right)} \\
& \times\left[\begin{array}{cc}
s-a_{22} & a_{12} \\
a_{21} & s-a_{11}
\end{array}\right] \\
& =\frac{1}{s^{2}-T s+\Delta}\left[\begin{array}{cc}
s-a_{22} & a_{12} \\
a_{21} & s-a_{11}
\end{array}\right] \text {. }
\end{aligned}
$$


In this section we study the local activity and local passivity of two-port and second-order cells by using $\mathbf{Z}_{Q}(s)$ and $\mathbf{Y}_{Q}(s)$. We also prove that the conclusions derived from $\mathbf{Z}_{Q}(s)$ and $\mathbf{Y}_{Q}(s)$ are equivalent.

\subsection{Passivity of two-port and second-order cells}

\subsubsection{Conclusions from $\mathbf{Z}_{Q}(s)$}

Let us find the parameter regions where properties $\mathrm{P} 1$ to $\mathrm{P} 3$ are satisfied in terms of $\mathbf{Z}_{Q}(s)$. $\mathrm{P} 1\left(\mathbf{Z}_{Q}(s)\right)$. Conditions for $\operatorname{Re}\left[s_{p}\right] \leq 0$ are given by

$$
T \leq 0 \quad \mathbb{A N D} \quad \Delta \geq 0 .
$$

$\mathrm{P} 2\left(\mathbf{Z}_{Q}(s)\right)$. Conditions for " $\mathbf{Z}_{Q}^{\dagger}(i \omega)+\mathbf{Z}_{Q}(i \omega)$ is positive semi-definite for all $\omega \in \mathbb{R}$ and $s=i \omega$ is not a pole of $\mathbf{Z}(s)$ " are found as follow:

$$
\begin{aligned}
\mathbf{Z}^{\dagger}(i \omega)+\mathbf{Z}(i \omega) & =\left[\begin{array}{cc}
2 \operatorname{Re}\left(\frac{i \omega-a_{22}}{-\omega^{2}-i T \omega+\Delta}\right) & \frac{a_{12}}{-\omega^{2}-i T \omega+\Delta}+\frac{a_{21}}{-\omega^{2}+i T \omega+\Delta} \\
\frac{a_{12}}{-\omega^{2}+i T \omega+\Delta}+\frac{a_{21}}{-\omega^{2}-i T \omega+\Delta} & 2 \operatorname{Re}\left(\frac{i \omega-a_{11}}{-\omega^{2}-i T \omega+\Delta}\right)
\end{array}\right] \\
& =\left[\begin{array}{cc}
2 \frac{-a_{22}\left(-\omega^{2}+\Delta\right)-T \omega^{2}}{\left(-\omega^{2}+\Delta\right)^{2}+T^{2} \omega^{2}} & \frac{\left(-\omega^{2}+\Delta\right)\left(a_{12}+a_{21}\right)+i T \omega\left(a_{12}-a_{21}\right)}{\left(-\omega^{2}+\Delta\right)^{2}+T^{2} \omega^{2}} \\
\frac{\left(-\omega^{2}+\Delta\right)\left(a_{12}+a_{21}\right)-i T \omega\left(a_{12}-a_{21}\right)}{\left(-\omega^{2}+\Delta\right)^{2}+T^{2} \omega^{2}} & 2 \frac{-a_{11}\left(-\omega^{2}+\Delta\right)-T \omega^{2}}{\left(-\omega^{2}+\Delta\right)^{2}+T^{2} \omega^{2}}
\end{array}\right] .
\end{aligned}
$$

Since the associated Hermitian matrix

$$
\left[\begin{array}{cc}
2\left(-a_{22} \Delta-a_{11} \omega^{2}\right) & \left(-\omega^{2}+\Delta\right)\left(a_{12}+a_{21}\right)+i T \omega\left(a_{12}-a_{21}\right) \\
\left(-\omega^{2}+\Delta\right)\left(a_{12}+a_{21}\right)-i T \omega\left(a_{12}-a_{21}\right) & 2\left(-a_{11} \Delta-a_{22} \omega^{2}\right)
\end{array}\right]
$$

must be positive semi-definite, it follows from Theorem 1 that the eigenvalues must be real and nonnegative. The eigenvalues of the above matrix are given by the solutions $\lambda_{1,2}$ of the following equation:

$$
\begin{aligned}
{[\lambda+} & \left.2\left(a_{22} \Delta+a_{11} \omega^{2}\right)\right]\left[\lambda+2\left(a_{11} \Delta+a_{22} \omega^{2}\right)\right] \\
& -\left(-\omega^{2}+\Delta\right)^{2}\left(a_{12}+a_{21}\right)^{2} \\
& -T^{2} \omega^{2}\left(a_{12}-a_{21}\right)^{2}=0
\end{aligned}
$$

The following three equations are used to organize and simplify the terms from this equation:

$$
\begin{aligned}
\lambda^{2}+ & 2\left[\left(a_{11}+a_{22}\right) \Delta+\left(a_{11} \omega^{2}+a_{22} \omega^{2}\right)\right] \lambda \\
& +4\left(a_{22} \Delta+a_{11} \omega^{2}\right)\left(a_{11} \Delta+a_{22} \omega^{2}\right) \\
& -\left(\omega^{2}-\Delta\right)^{2}\left(a_{12}+a_{21}\right)^{2} \\
& -T^{2} \omega^{2}\left(a_{12}-a_{21}\right)^{2}=0 .
\end{aligned}
$$

$$
\begin{aligned}
\lambda^{2}+ & 2 T\left(\Delta+\omega^{2}\right) \lambda+4 a_{11} a_{22} \omega^{4}+4\left(a_{11}^{2}\right. \\
& \left.+a_{22}^{2}\right) \Delta \omega^{2}+4 a_{11} a_{22} \Delta^{2}-\left(a_{12}+a_{21}\right)^{2} \omega^{4} \\
& +2 \Delta\left(a_{12}+a_{21}\right)^{2} \omega^{2}-\left(a_{12}+a_{21}\right)^{2} \Delta^{2} \\
& -T^{2} \omega^{2}\left(a_{12}-a_{21}\right)^{2}=0 \\
\lambda^{2}+ & 2 T\left(\Delta+\omega^{2}\right) \lambda+\left[4 a_{11} a_{22}-\left(a_{12}+a_{21}\right)^{2}\right] \omega^{4} \\
& +4\left[\left(a_{11}^{2}+a_{22}^{2}\right) \Delta+2\left(a_{12}+a_{21}\right)^{2} \Delta\right. \\
& \left.-T^{2}\left(a_{12}-a_{21}\right)^{2}\right] \omega^{2}+\left[4 a_{11} a_{22}\right. \\
& \left.-\left(a_{12}+a_{21}\right)^{2}\right] \Delta^{2}=0 .
\end{aligned}
$$

Defining

$$
\begin{aligned}
U \triangleq & 2 T\left(\Delta+\omega^{2}\right), \\
V \triangleq & {\left[4 a_{11} a_{22}-\left(a_{12}+a_{21}\right)^{2}\right] \omega^{4}+\left[4\left(a_{11}^{2}+a_{22}^{2}\right) \Delta\right.} \\
& \left.+2\left(a_{12}+a_{21}\right)^{2} \Delta-T^{2}\left(a_{12}-a_{21}\right)^{2}\right] \omega^{2} \\
& +\left[4 a_{11} a_{22}-\left(a_{12}+a_{21}\right)^{2}\right] \Delta^{2},
\end{aligned}
$$


we can recase Eq. (67) as follow:

$$
\lambda^{2}+U \lambda+V=0 .
$$

It follows that $\lambda_{1} \geq 0 \quad \mathbb{A N D} \quad \lambda_{2} \geq 0$ for all $\omega \in \mathbb{R}$ if, and only if,

$$
U \leq 0 \quad \mathbb{A N D} \quad V \geq 0, \text { for all } \omega \in \mathbb{R} .
$$

Let us analyze the composite conditions in Eq. (70) as follow:

Case 1. $U \leq 0$.

From $U \leq 0$ for all $\omega \in \mathbb{R}$ we have

$$
T\left(\Delta+\omega^{2}\right) \leq 0 \text { for all } \omega \in \mathbb{R}
$$

from which we obtain the simple condition

$$
T \leq 0 \quad \mathbb{A N D} \quad \Delta \geq 0 .
$$

Case 2. $\quad V \geq 0$.

From $V \geq 0$ for all $\omega \in \mathbb{R}$ we have

$$
4 a_{11} a_{22}-\left(a_{12}+a_{21}\right)^{2} \geq 0
$$

where the minimum value $V\left(\omega_{\min }\right)$ of $V(\omega)$ must be non-negative and $\omega_{\min }$ is given by

$$
\omega_{\min }^{2}= \begin{cases}0, & \text { if } K \leq 0, \\ K, & \text { otherwise }\end{cases}
$$

where

$$
\begin{aligned}
& 4\left(a_{11}^{2}+a_{22}^{2}\right) \Delta+2\left(a_{12}+a_{21}\right)^{2} \Delta \\
& K=-\frac{-T^{2}\left(a_{12}-a_{21}\right)^{2}}{2\left[4 a_{11} a_{22}-\left(a_{12}+a_{21}\right)^{2}\right]} \text {. }
\end{aligned}
$$

If $\omega_{\min }^{2}=0$ we have

$$
\begin{gathered}
4 a_{11} a_{22}-\left(a_{12}+a_{21}\right)^{2} \geq 0 \quad \mathbb{A N D} \\
{\left[4 a_{11} a_{22}-\left(a_{12}+a_{21}\right)^{2}\right] \Delta^{2} \geq 0 \quad \mathbb{A N D}} \\
4\left(a_{11}^{2}+a_{22}^{2}\right) \Delta+2\left(a_{12}+a_{21}\right)^{2} \Delta \\
-T^{2}\left(a_{12}-a_{21}\right)^{2} \geq 0
\end{gathered}
$$

which is the same as

$$
\begin{aligned}
& 4 a_{11} a_{22}-\left(a_{12}+a_{21}\right)^{2} \geq 0 \quad \mathbb{A N D} \\
& 4\left(a_{11}^{2}+a_{22}^{2}\right) \Delta+2\left(a_{12}+a_{21}\right)^{2} \Delta \\
& -T^{2}\left(a_{12}-a_{21}\right)^{2} \geq 0 .
\end{aligned}
$$

If $\omega_{\max }^{2}=K$ we have

$$
\begin{aligned}
& 4 a_{11} a_{22}-\left(a_{12}+a_{21}\right)^{2} \geq 0 \quad \mathbb{A N D} \quad-\frac{4\left(a_{11}^{2}+a_{22}^{2}\right) \Delta+2\left(a_{12}+a_{21}\right)^{2} \Delta-T^{2}\left(a_{12}-a_{21}\right)^{2}}{4\left[4 a_{11} a_{22}-\left(a_{12}+a_{21}\right)^{2}\right]} \\
& +\left[4 a_{11} a_{22}-\left(a_{12}+a_{21}\right)^{2}\right] \Delta^{2} \geq 0 \quad \text { AND } \quad 4\left(a_{11}^{2}+a_{22}^{2}\right) \Delta+2\left(a_{12}+a_{21}\right)^{2} \Delta-T^{2}\left(a_{12}-a_{21}\right)^{2}<0 .
\end{aligned}
$$

The above results are rather messy. To simplify the results, let us apply the first condition of Theorem 2 and obtain

$$
-a_{22} \Delta-a_{11} \omega^{2} \geq 0, \quad \text { for all } \omega \in \mathbb{R}
$$

It follows that

$$
a_{11} \leq 0 \quad \mathbb{A N D} \quad a_{22} \Delta \leq 0 .
$$

Combining the results in Eqs. (78) and (72) we have

$$
a_{11} \leq 0 \quad \mathbb{A N D} \quad a_{22} \leq 0 \quad \mathbb{A N D} \quad \Delta \geq 0 .
$$

Applying the second condition of Theorem 2, we obtain

$$
\begin{gathered}
4\left(a_{22} \Delta+a_{11} \omega^{2}\right)\left(a_{11} \Delta+a_{22} \omega^{2}\right) \\
-\left(\Delta-\omega^{2}\right)^{2}\left(a_{12}+a_{21}\right)^{2} \\
-\left(a_{12}-a_{21}\right)^{2} T^{2} \omega^{2} \geq 0
\end{gathered}
$$

which leads to

$$
\begin{gathered}
{\left[4 a_{11} a_{22}-\left(a_{12}+a_{21}\right)^{2}\right] \omega^{4}+\left[4\left(a_{11}^{2}+a_{22}^{2}\right) \Delta\right.} \\
\left.+2\left(a_{12}+a_{21}\right)^{2} \Delta-T^{2}\left(a_{12}-a_{21}\right)^{2}\right] \omega^{2} \\
+\left[4 a_{11} a_{22}-\left(a_{12}+a_{21}\right)^{2}\right] \Delta^{2} \geq 0 .
\end{gathered}
$$

Comparing with Eq. (68) we observe that this is exactly the condition $V \geq 0$. Therefore this condition offers no new information.

P3 $\left(Z_{Q}(s)\right)$. Conditions for " $Z_{Q}(s)$ has a simple pole at $s_{p}=i \omega$ and its associated residue matrix $\mathbf{K}_{-1}$ is a positive semi-definite Hermitian matrix." In this case

$$
T=0, \Delta>0, s_{1,2}= \pm i \sqrt{\Delta} .
$$


The residue matrix is given by

$$
\begin{aligned}
\mathbf{K}_{-1} & =\lim _{s \rightarrow \pm i \sqrt{\Delta}} \frac{1}{s \mp i \sqrt{\Delta}} \frac{1}{s^{2}+\Delta}\left[\begin{array}{cc}
s-a_{22} & a_{12} \\
a_{21} & s-a_{11}
\end{array}\right] \\
& =\frac{1}{ \pm i 2 \sqrt{\Delta}}\left[\begin{array}{cc} 
\pm i \sqrt{\Delta}-a_{22} & a_{12} \\
a_{21} & \pm i \sqrt{\Delta}-a_{11}
\end{array}\right]
\end{aligned}
$$

which is positive semi-definite Hermitian if

$$
a_{22}=a_{11}=0 \quad \mathbb{A N D} \quad a_{12}=-a_{21}=0 .
$$

\subsubsection{Conclusions from $\mathbf{Y}_{Q}(s)$}

Let us derive next the parameter regions where properties $\mathrm{P} 1$ to $\mathrm{P} 3$ are satisfied in terms of

$$
\mathbf{Y}_{Q}(s)=\left[\begin{array}{cc}
s-a_{11} & -a_{12} \\
-a_{21} & s-a_{22}
\end{array}\right]
$$

$\mathrm{P} 1\left(\mathbf{Y}_{Q}(s)\right)$. Conditions for "Re $\left[s_{p}\right] \leq 0$."

Observe that $Y_{Q}(s)$ does not have poles in the finite $s$-plane. At the infinite pole we have the residue matrix

$$
\mathbf{K}_{-1}=\lim _{\omega \rightarrow \infty} \frac{\mathbf{Y}(i \omega)}{i \omega}=\left[\begin{array}{ll}
1 & 0 \\
0 & 1
\end{array}\right]
$$

which is positive definite.

$\mathrm{P} 2\left(\mathbf{Y}_{Q}(s)\right)$. Conditions for " $\mathbf{Y}_{Q}^{\dagger}(i \omega)+\mathbf{Y}_{Q}(i \omega)$ is positive semi-definite for all $\omega \in \mathbb{R}$ and $s=$ $i \omega$ is not a pole of $\mathbf{Y}_{Q}(s)$ " are evaluated as follows:

$$
\begin{aligned}
\mathbf{Y}_{Q}^{\dagger}(i \omega) & +\mathbf{Y}_{Q}(i \omega) \\
= & {\left[\begin{array}{cc}
-i \omega-a_{11} & -a_{21} \\
-a_{12} & -i \omega-a_{22}
\end{array}\right] } \\
& +\left[\begin{array}{cc}
i \omega-a_{11} & -a_{12} \\
-a_{21} & i \omega-a_{22}
\end{array}\right] \\
= & {\left[\begin{array}{cc}
-2 a_{11} & -a_{12}-a_{21} \\
-a_{12}-a_{21} & -2 a_{22}
\end{array}\right] . }
\end{aligned}
$$

Applying Theorem 1, the condition for the above symmetric matrix to be positive semi-definite is that all eigenvalues must be non-negative. The eigenvalues are given by the solutions of the equation:

$$
\left|\begin{array}{cc}
\lambda+2 a_{11} & a_{12}+a_{21} \\
a_{12}+a_{21} & \lambda+2 a_{22}
\end{array}\right|=0
$$

which leads to

$$
\begin{array}{r}
\lambda^{2}+2\left(a_{11}+a_{22}\right) \lambda+4 a_{11} a_{22} \\
-\left(a_{12}+a_{21}\right)^{2}=0 .
\end{array}
$$

Observe that all roots of Eq. (91) are non-negative if, and only if,

$$
\begin{gathered}
2\left(a_{11}+a_{22}\right) \leq 0 \quad \mathbb{A N D} \quad 4 a_{11} a_{22} \\
-\left(a_{12}+a_{21}\right)^{2} \geq 0 .
\end{gathered}
$$

The above conditions are equivalent to

$$
\begin{gathered}
T \leq 0 \quad \mathbb{A N D} \quad 2 \Delta+2 a_{11} a_{22} \\
-a_{12}^{2}-a_{21}^{2} \geq 0 .
\end{gathered}
$$

Applying the first condition of Theorem 2 we obtain

$$
a_{11} \leq 0 .
$$

The second condition of Theorem 2 leads to

$$
4 a_{11} a_{22}-\left(a_{12}+a_{21}\right)^{2} \geq 0 .
$$

$\mathrm{P} 3\left(\mathbf{Y}_{Q}(s)\right)$. Conditions for "Y $\mathbf{Y}_{Q}(s)$ has a simple pole at $s_{p}=i \omega$ and its associated residue matrix $\mathbf{K}_{-1}$ is a positive semi-definite Hermitian matrix".

In this case,

$$
\begin{aligned}
\mathbf{K}_{-1} & =\lim _{\omega \rightarrow \infty} \frac{1}{i \omega}\left[\begin{array}{cc}
i \omega-a_{11} & -a_{12} \\
-a_{21} & i \omega-a_{22}
\end{array}\right] \\
& =\left[\begin{array}{ll}
1 & 0 \\
0 & 1
\end{array}\right]
\end{aligned}
$$

which is a positive semi-definite Hermitian matrix for all parameters. Therefore this criterion provides no additional information.

\subsubsection{Comparing the results from $\mathbf{Z}_{Q}(s)$ and $\mathbf{Y}_{Q}(s)$}

Let us now prove that the local passive regions derived from $\mathbf{Z}_{Q}(s)$ and $\mathbf{Y}_{Q}(s)$ are identical. 
1. The local passive regions derived from $\mathbf{Z}_{Q}(s)$ are given by

$$
\begin{aligned}
& a_{11} \leq 0 \quad \mathbb{A N D} \quad a_{22} \leq 0 \quad \mathbb{A N D} \quad 4 a_{11} a_{22}-\left(a_{12}+a_{21}\right)^{2} \geq 0 \quad \mathbb{A N D} \quad \Delta \geq 0 \quad \mathbb{A N D} \quad\left\{4\left(a_{11}^{2}+a_{22}^{2}\right) \Delta\right. \\
& +2\left(a_{12}+a_{21}\right)^{2} \Delta-T^{2}\left(a_{12}-a_{21}\right)^{2} \geq 0 \quad \mathbb{O} \mathbb{R} \quad\left[-\frac{4\left(a_{11}^{2}+a_{22}^{2}\right) \Delta+2\left(a_{12}+a_{21}\right)^{2} \Delta-T^{2}\left(a_{12}-a_{21}\right)^{2}}{4\left[4 a_{11} a_{22}-\left(a_{12}+a_{21}\right)^{2}\right]}\right. \\
& \left.\left.+\left[4 a_{11} a_{22}-\left(a_{12}+a_{21}\right)^{2}\right] \Delta^{2} \geq 0 \quad \mathbb{A N D} \quad 4\left(a_{11}^{2}+a_{22}^{2}\right) \Delta+2\left(a_{12}+a_{21}\right)^{2} \Delta-T^{2}\left(a_{12}-a_{21}\right)^{2}<0\right]\right\}
\end{aligned}
$$

2. The local passive regions derived from $\mathbf{Y}_{Q}(s)$ are given by

$$
a_{11} \leq 0 \quad \mathbb{A N D} \quad a_{11}+a_{22} \leq 0 \quad \mathbb{A N D} \quad 4 a_{11} a_{22}-\left(a_{12}+a_{21}\right)^{2} \geq 0
$$

Observe that $a_{22}>0$ is impossible, for otherwise it would follow from $a_{11} \leq 0$ that $4 a_{11} a_{22} \leq 0$, contradicting the condition $4 a_{11} a_{22}-\left(a_{12}+a_{21}\right)^{2} \geq 0$. Therefore Eq. (98) can be rewritten as

Local Passivity

Criteria

$$
a_{11} \leq 0 \quad \mathbb{A N D} \quad a_{22} \leq 0 \quad \mathbb{A N D} \quad 4 a_{11} a_{22}-\left(a_{12}+a_{21}\right)^{2} \geq 0 .
$$

Theorem 3. The conditions in Eqs. (97) and (99) are equivalent.

Proof. Let us first simplify the conditions in Eq. (97) as follows.

$$
\begin{aligned}
4 \Delta & =2 \Delta+2 \Delta \\
& =2\left(a_{11} a_{22}-a_{12} a_{21}\right)+2\left(a_{11} a_{22}-a_{12} a_{21}\right) \\
& \geq 2\left(a_{11} a_{22}-a_{12} a_{21}\right)+2 a_{11} a_{22}-\left(a_{12}^{2}+a_{21}^{2}\right) \\
& =4 a_{11} a_{22}-\left(a_{12}^{2}-2 a_{12} a_{21}+a_{21}^{2}\right) \\
& =4 a_{11} a_{22}-\left(a_{12}+a_{21}\right)^{2} .
\end{aligned}
$$

It follows from the third inequality in Eq. (97) and Eq. (100) that $4 \Delta \geq 0$, which leads to the fourth inequality in Eq. (97). Therefore the fourth inequality in Eq. (97) is redundant and Eq. (97) can be rewritten as

$$
\begin{aligned}
a_{11} \leq & 0 \quad \mathbb{A N D} \quad a_{22} \leq 0 \quad \mathbb{A N D} \quad 4 a_{11} a_{22}-\left(a_{12}+a_{21}\right)^{2} \geq 0 \quad \mathbb{A N D} \quad\left\{4\left(a_{11}^{2}+a_{22}^{2}\right) \Delta+2\left(a_{12}+a_{21}\right)^{2} \Delta\right. \\
& -T^{2}\left(a_{12}-a_{21}\right)^{2} \geq 0 \quad \mathbb{O} \mathbb{R} \quad\left[\begin{array}{lll}
-\frac{4\left(a_{11}^{2}+a_{22}^{2}\right) \Delta+2\left(a_{12}+a_{21}\right)^{2} \Delta-T^{2}\left(a_{12}-a_{21}\right)^{2}}{4\left[4 a_{11} a_{22}-\left(a_{12}+a_{21}\right)^{2}\right]} \\
+
\end{array}\right. \\
& \left.\left.+\left[\begin{array}{llll}
4 a_{11} a_{22}-\left(a_{12}+a_{21}\right)^{2}
\end{array}\right] \Delta^{2} \geq 0 \quad \mathbb{A N D} \quad 4\left(a_{11}^{2}+a_{22}^{2}\right) \Delta+2\left(a_{12}+a_{21}\right)^{2} \Delta-T^{2}\left(a_{12}-a_{21}\right)^{2}<0\right]\right\} .
\end{aligned}
$$

If

$$
4\left(a_{11}^{2}+a_{22}^{2}\right) \Delta+2\left(a_{12}+a_{21}\right)^{2} \Delta-T^{2}\left(a_{12}-a_{21}\right)^{2}<0
$$


and

$$
4 a_{11} a_{22}-\left(a_{12}+a_{21}\right)^{2} \geq 0
$$

then we have

$$
-\frac{4\left(a_{11}^{2}+a_{22}^{2}\right) \Delta+2\left(a_{12}+a_{21}\right)^{2} \Delta-T^{2}\left(a_{12}-a_{21}\right)^{2}}{4\left[4 a_{11} a_{22}-\left(a_{12}+a_{21}\right)^{2}\right]}+\left[4 a_{11} a_{22}-\left(a_{12}+a_{21}\right)^{2}\right] \Delta^{2} \geq 0 .
$$

It follows from the above conclusion that the condition

$$
\begin{aligned}
& {\left[-\frac{4\left(a_{11}^{2}+a_{22}^{2}\right) \Delta+2\left(a_{12}+a_{21}\right)^{2} \Delta-T^{2}\left(a_{12}-a_{21}\right)^{2}}{4\left[4 a_{11} a_{22}-\left(a_{12}+a_{21}\right)^{2}\right]}+\left[4 a_{11} a_{22}-\left(a_{12}+a_{21}\right)^{2}\right] \Delta^{2}\right.} \\
& \left.\left.\quad \geq 0 \quad \mathbb{A N D} \quad 4\left(a_{11}^{2}+a_{22}^{2}\right) \Delta+2\left(a_{12}+a_{21}\right)^{2} \Delta-T^{2}\left(a_{12}-a_{21}\right)^{2}<0\right]\right\}
\end{aligned}
$$

can be reduced to

$$
4\left(a_{11}^{2}+a_{22}^{2}\right) \Delta+2\left(a_{12}+a_{21}\right)^{2} \Delta-T^{2}\left(a_{12}-a_{21}\right)^{2}<0 .
$$

Finally, the condition

$$
\begin{aligned}
& \left\{4\left(a_{11}^{2}+a_{22}^{2}\right) \Delta+2\left(a_{12}+a_{21}\right)^{2} \Delta-T^{2}\left(a_{12}-a_{21}\right)^{2} \geq 0 \quad \mathbb{O} \mathbb{R}\right. \\
& \quad\left[-\frac{4\left(a_{11}^{2}+a_{22}^{2}\right) \Delta+2\left(a_{12}+a_{21}\right)^{2} \Delta-T^{2}\left(a_{12}-a_{21}\right)^{2}}{4\left[4 a_{11} a_{22}-\left(a_{12}+a_{21}\right)^{2}\right]}+\left[4 a_{11} a_{22}-\left(a_{12}+a_{21}\right)^{2}\right] \Delta^{2}\right. \\
& \left.\left.\quad \geq 0 \quad \mathbb{A N D} \quad 4\left(a_{11}^{2}+a_{22}^{2}\right) \Delta+2\left(a_{12}+a_{21}\right)^{2} \Delta-T^{2}\left(a_{12}-a_{21}\right)^{2}<0\right]\right\}
\end{aligned}
$$

becomes

$$
\begin{aligned}
& 4\left(a_{11}^{2}+a_{22}^{2}\right) \Delta+2\left(a_{12}+a_{21}\right)^{2} \Delta \\
& \quad-T^{2}\left(a_{12}-a_{21}\right)^{2} \geq 0 \quad \mathbb{O R} \\
& 4\left(a_{11}^{2}+a_{22}^{2}\right) \Delta+2\left(a_{12}+a_{21}\right)^{2} \Delta \\
& -T^{2}\left(a_{12}-a_{21}\right)^{2}<0
\end{aligned}
$$

which is always true regardless of the choice of parameters. Therefore, as a final conclusion, the conditions in Eq. (97) are equivalent to

$$
\begin{gathered}
a_{11} \leq 0 \quad \mathbb{A N D} \quad a_{22} \leq 0 \quad \mathbb{A N D} \quad 4 a_{11} a_{22} \\
-\left(a_{12}+a_{21}\right)^{2} \geq 0
\end{gathered}
$$

which is exactly Eq. (99). This completes our proof that Eq. (97) $\Rightarrow$ Eq. (99). Since all deductions presented so far are reversible, if follows that Eq. (99) $\Rightarrow(97)$.

Remark. It follows from Eq. (99) that a two-port and second-order cell is locally active at equilibrium point $Q$ if, and only if, any one of the three inequalities in Eq. (99) is violated, which is precisely Corollary 4.4.3 in p. 296 of [Chua, 1998].

\subsection{Active regions for two-port and second-order CNN Cells}

For illustrative purposes, let us derive next the following equivalent conditions for local activity.

$\mathrm{A} 1\left(\mathbf{Z}_{Q}(s)\right)$. Conditions for " $\operatorname{Re}\left[s_{p}\right]>0$ " are those for $\mathbb{N O T}\left(\mathrm{P} 1\left(\mathbf{Z}_{Q}(s)\right)\right.$ and are given by

$$
T>0 \quad \mathbb{O} \mathbb{R} \quad \Delta<0 .
$$

$\mathrm{A} 2\left(\mathbf{Z}_{Q}(s)\right)$. Conditions for "NOT $\left(\mathbf{P} 2\left(\mathbf{Z}_{Q}(s)\right)\right)$ " satisfy

$$
A 2\left(\mathbf{Z}_{Q}(s)\right) \subseteq A 1\left(\mathbf{Z}_{Q}(s)\right)
$$

Therefore, for the purpose of studying the local activity parameter regions, $\mathrm{A} 2\left(\mathbf{Z}_{Q}(s)\right)$ contributes no new information. 
$\mathrm{A} 3\left(\mathbf{Z}_{Q}(s)\right)$. Conditions for " $\mathbf{Z}_{Q}(s)$ has a simple pole $s_{p}= \pm i \omega$ whose residue matrix $\mathbf{K}_{-1}$ is either not Hermitian, or is not positive semi-definite" are given by

$$
\begin{gathered}
T=0 \quad \mathbb{A N D} \quad \Delta>0 \quad \mathbb{A N D} \\
{\left[a_{11} \neq 0 \quad \mathbb{O} \mathbb{R} \quad a_{22} \neq 0 \quad \mathbb{O} \mathbb{R}\right.} \\
\left.a_{12} \neq 0 \quad \mathbb{O} \mathbb{R} \quad a_{21} \neq 0\right] .
\end{gathered}
$$

$\mathrm{A} 4\left(\mathbf{Z}_{Q}(s)\right)$. Conditions for " $\mathbf{Z}_{Q}(s)$ has a multiple pole $s_{p}= \pm i \omega "$ are given by

$$
\begin{gathered}
T=0 \quad \mathbb{A N D} \quad \Delta=0 \quad \mathbb{A N D} \\
{\left[a_{11} \neq 0 \quad \mathbb{O} \mathbb{R} \quad a_{22} \neq 0\right] .}
\end{gathered}
$$

\section{One-Port and First-Order CNN Cells: Examples}

As an illustrative example, consider the state equation of a one-port first-order cell given by

$$
\dot{V}_{1}=-V_{1}^{3}+\alpha V_{1}+I^{*}
$$

where $I^{*} \in(-\infty, \infty)$ is an external constant called the "cell bias". An equilibrium point $Q$ is obtained by solving the equation

$$
V_{1}^{* 3}-\alpha V_{1}^{*}-I^{*}=0
$$

as a function of $I^{*}$ and $\alpha$. Depending on the value

$$
\mathfrak{D} \triangleq \frac{I^{* 2}}{4}-\frac{\alpha^{3}}{27}
$$

Eq. (112) can have one, two, or three distinct solutions; namely,

1. $\mathfrak{D}>0$ : only one equilibrium point

$$
V_{1}^{*}=\left(\frac{I^{*}}{2}+\sqrt{\mathfrak{D}}\right)^{1 / 3}+\left(\frac{I^{*}}{2}-\sqrt{\mathfrak{D}}\right)^{1 / 3} .
$$

2. $\mathfrak{D}=0$ : two equilibrium points

$$
V_{1 a}^{*}=2\left(\frac{I^{*}}{2}\right)^{1 / 3}, V_{1 b}^{*}=\left(\frac{I^{*}}{2}\right)^{1 / 3} .
$$

3. $\mathfrak{D}<0$ : three equilibrium points

$$
\begin{aligned}
V_{1 a}^{*} & =2 \sqrt{\frac{|\alpha|}{3}} \cos \frac{\psi}{3}, \\
V_{1 b}^{*} & =-2 \sqrt{\frac{|\alpha|}{3}} \cos \left(\frac{\psi}{3}-\frac{\pi}{3}\right), \\
V_{1 c}^{*} & =-2 \sqrt{\frac{|\alpha|}{3}} \cos \left(\frac{\psi}{3}+\frac{\pi}{3}\right)
\end{aligned}
$$

where

$$
\psi=\cos ^{-1}\left(\frac{I^{*} / 2}{\sqrt{|\alpha|^{3} / 27}}\right)
$$

The linearized cell state equation about each equilibrium point is given by

$$
\dot{v}_{1}=\left(-3 V_{1}^{* 2}+\alpha\right) v_{1}+i_{1} .
$$

where $v_{1}$ and $i_{1}$ denote small perturbations about $V_{1}^{*}$ and $I^{*}$, respectively.

The local passive parameter region at each equilibrium point $Q=\left(V_{1}^{*}, I^{*}\right)^{\top}$ is given by

$$
\alpha \leq 3 V_{1}^{* 2}
$$

which depends on the positions $V_{1}^{*}$ of the equilibrium point. Since there are three different cases, we need to consider them separately as follows.

1. $\mathfrak{D}>0$. The local passive regions are given by

$$
\begin{aligned}
\frac{I^{* 2}}{4}- & \frac{\alpha^{3}}{27}>0 \\
& -3\left[\left(\frac{I^{*}}{2}+\sqrt{\mathfrak{D}}\right)^{1 / 3}+\left(\frac{I^{*}}{2}-\sqrt{\mathfrak{D}}\right)^{1 / 3}\right]^{2} \\
& +\alpha \leq 0
\end{aligned}
$$

Since the second inequality is always satisfied if the first inequality is satisfied, it follows that

$$
\frac{I^{* 2}}{4}-\frac{\alpha^{3}}{27}>0
$$

defines the local passive parameter region.

2. $\mathfrak{D}=0$. Since there are two equilibrium points in this degenerate case, the local passive regions are defined by the set intersection of the following parameter regions associated with the two equilibrium points

$$
\begin{aligned}
\frac{I^{* 2}}{4} & -\frac{\alpha^{3}}{27}=0, \\
& -12\left(\frac{I^{*}}{2}\right)^{2 / 3}+\alpha \leq 0 \quad \mathbb{A N D} \\
& -3\left(\frac{I^{*}}{2}\right)^{2 / 3}+\alpha \leq 0 .
\end{aligned}
$$


Solving for $\alpha$, we obtain

$$
\begin{aligned}
& \alpha=3\left(\frac{I^{*}}{2}\right)^{2 / 3}, \\
& \alpha \leq 3\left(\frac{I^{*}}{2}\right)^{2 / 3} .
\end{aligned}
$$

It follows that the local passive region for $\mathfrak{D}=0$ consists of an isolated curve

$$
\alpha=3\left(\frac{I^{*}}{2}\right)^{2 / 3} .
$$

3. $\mathfrak{D}<0$. Since there are there equilibrium points in this case, the local passive regions are the intersection of the corresponding local passive parameter regions; namely,

$$
\begin{aligned}
\frac{I^{* 2}}{4}- & \frac{\alpha^{3}}{27}<0, \\
& -3(2 \sqrt{|\alpha| / 3} \cos (\psi / 3))^{2}+\alpha \leq 0 \quad \mathbb{A N D} \\
& -3(-2 \sqrt{|\alpha| / 3} \cos (\psi / 3-\pi / 3))^{2}+\alpha \leq 0 \\
& \mathbb{A N D} \\
& -3(-2 \sqrt{|\alpha| / 3} \cos (\psi / 3+\pi / 3))^{2}+\alpha \leq 0 .
\end{aligned}
$$

We will now show that the intersection of the local passive regions at these three equilibrium points is empty. Let us assume

$$
\frac{I^{*}}{2}=(1-\delta) \sqrt{\left(\frac{|\alpha|}{3}\right)^{3}}
$$

where $0<\delta \ll 1$, then $0<\psi \ll \pi / 2$. The intersection of the local passive regions at these three equilibrium points is given by

$$
\begin{aligned}
\alpha & \leq 3(-2 \sqrt{|\alpha| / 3} \cos (\psi / 3+\pi / 3))^{2} \\
& =3\left(-2 \sqrt{|\alpha| / 3}\left(1 / 2-\delta_{1}\right)\right)^{2} \\
& =|\alpha|\left(1-2 \delta_{1}\right)^{2}
\end{aligned}
$$

where $0<\delta_{1} \ll 1$. Since $\alpha>0$ when $\mathfrak{D}<0$, it follows that the above condition gives an empty set.
In Fig. 1 the locally passive, restricted locally passive and active parameter regions in the $I^{*}-\alpha$ plane are identified in blue, cyan and green colors, respectively. Observe that for $\alpha \leq 0$ (blue region) the cell is locally passive since the local passivity criteria are satisfied for $-\infty<I^{*}<\infty$. In contrast, for $0<\alpha<3\left(I^{*} / 2\right)^{2 / 3}$ (cyan region) the local passivity criteria hold only for certain ranges of $I^{*}$. The boundary curve separating the cyan and green regions (defined by $\mathfrak{D}=0$ ) also belong to the restricted local passive region. Notice that there is no edge-of-chaos region in this case.

We now present our simulation results for an $N \times N$ CNN denoted by the following equations:

$$
\begin{aligned}
\dot{V}_{1}(i, j)= & -V_{1}^{3}(i, j)+\alpha V_{1}(i, j)+D_{1}\left[V_{1}(i-1, j)\right. \\
& +V_{1}(i, j-1)+V_{1}(i, j+1) \\
& \left.+V_{1}(i+1, j)-4 V_{1}(i, j)\right] \\
& 1 \leq i \leq N, 1 \leq j \leq N
\end{aligned}
$$

The simulation results are shown in Fig. 2. Figure 2(a) shows a random initial condition. Figures 2(b) and 2(c) show the evolving process of the CNN with $D_{1}=1$ and $\alpha=-2$. As shown in Fig. 1, in this case the CNN is locally passive. From Figs. 2(b) and 2(c) we can see that the CNN approaches a homogeneous output. Figures $2(\mathrm{~d})$ and 2 (e) show the evolving process of the CNN with $D_{1}=1$ and $\alpha=2$. As shown in Fig. 1, in this case the CNN is locally active. From Figs. 2(d) and 2(e) we can see that the CNN outputs a static pattern.

In fact, these two kinds of patterns are the typical ones exhibited by this CNN. Observe that this CNN has a relatively low possibility for generating different kinds of patterns. We conjecture that this fact is due to the relatively simple configurations of different kinds of parameter regions in the $I^{*}$ - $\alpha$ plane.

\section{One-Port and Second-Order CNN Cells: Examples}

In this section let us study the CNN consisting of one-port second-order cells whose equations are given by

$$
\begin{aligned}
\dot{V}_{1}= & -V_{1}^{3}+(0.5 \alpha-b) V_{1}-V_{2}^{3} \\
& +(1.5 \alpha+b) V_{2}+2 I^{*}, \\
\dot{V}_{2}= & V_{1}-V_{2} .
\end{aligned}
$$




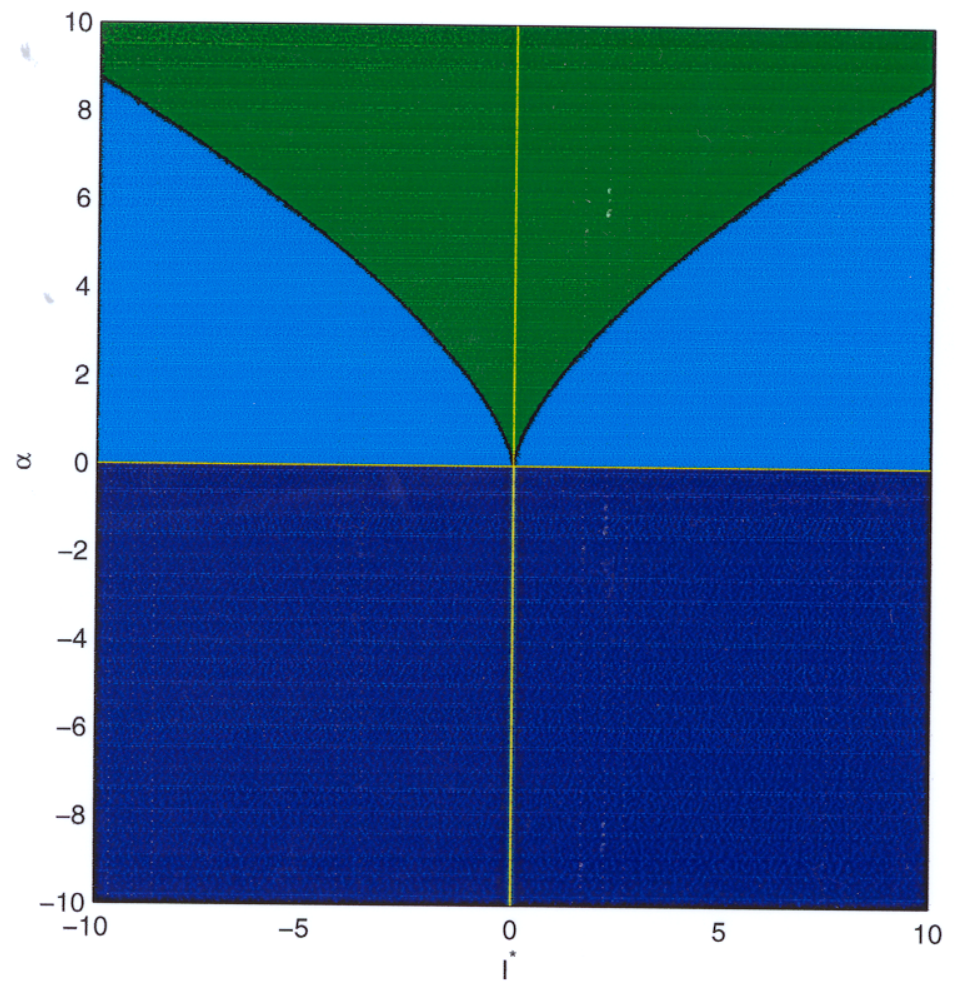

Fig. 1. Locally passive, restricted locally passive and locally active parameter regions for one-port first-order cells. There is no edge-of-chaos region.

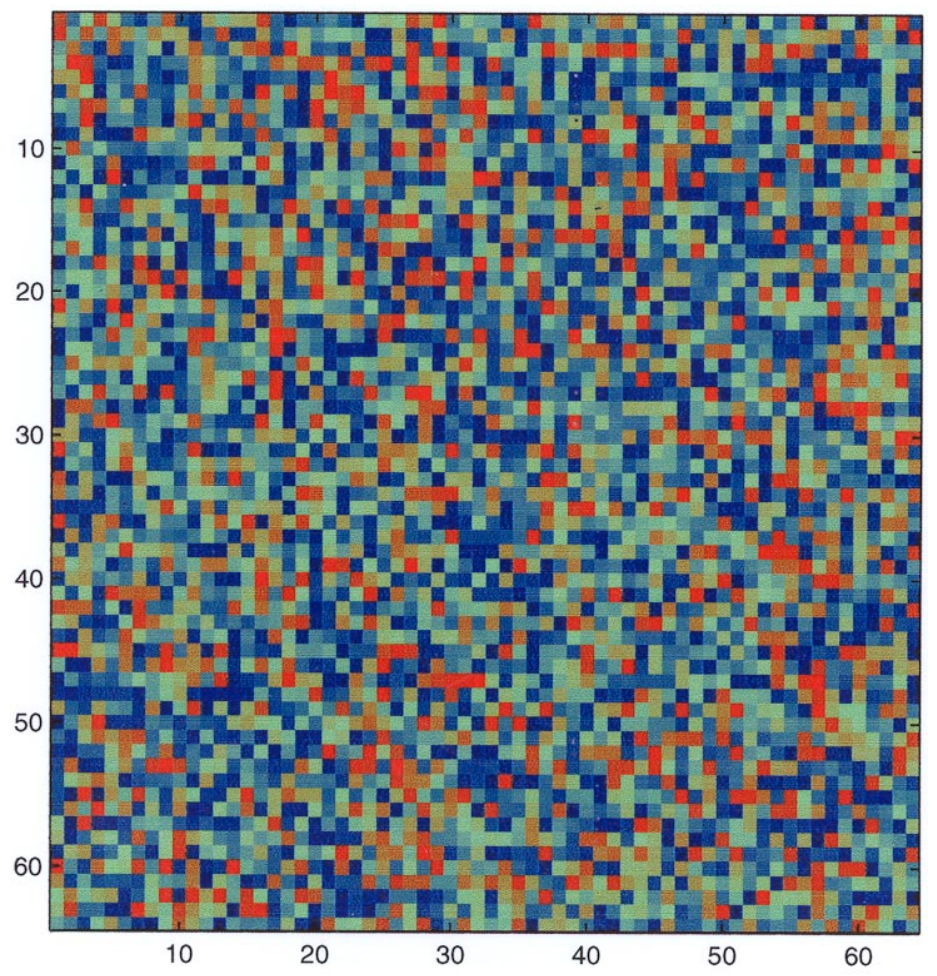

(a)

Fig. 2. The simulation results of a $64 \times 64 \mathrm{CNN}$ made of one-port first-order cells. The parameter $D_{1}=1$ is fixed. (a) Initial condition. (b) $\alpha=-2, t=0.1$, locally passive. (c) $\alpha=-2, t=1$, locally passive. (d) $\alpha=2, t=1$, locally active. (e) $\alpha=2$, $t=5$, locally active. 


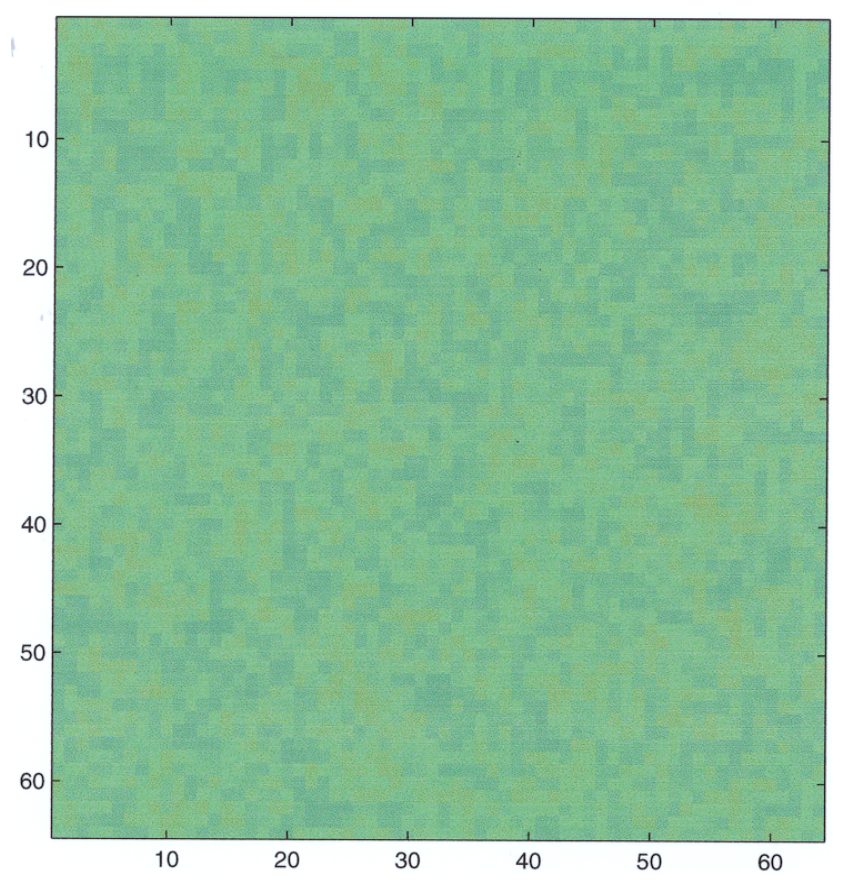

(b)

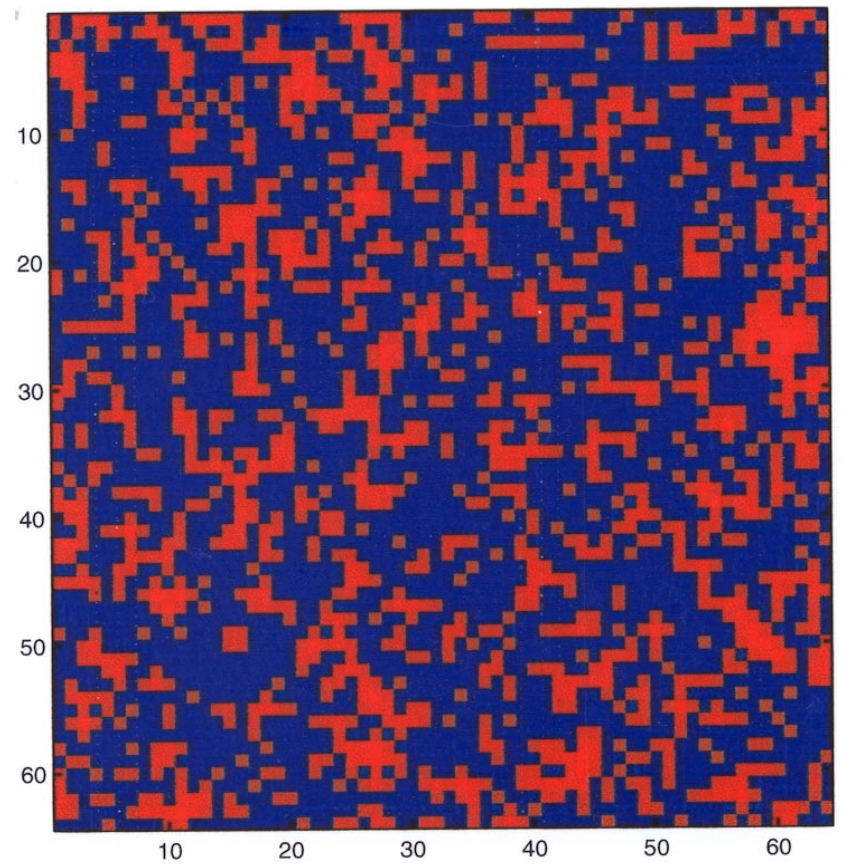

(d)

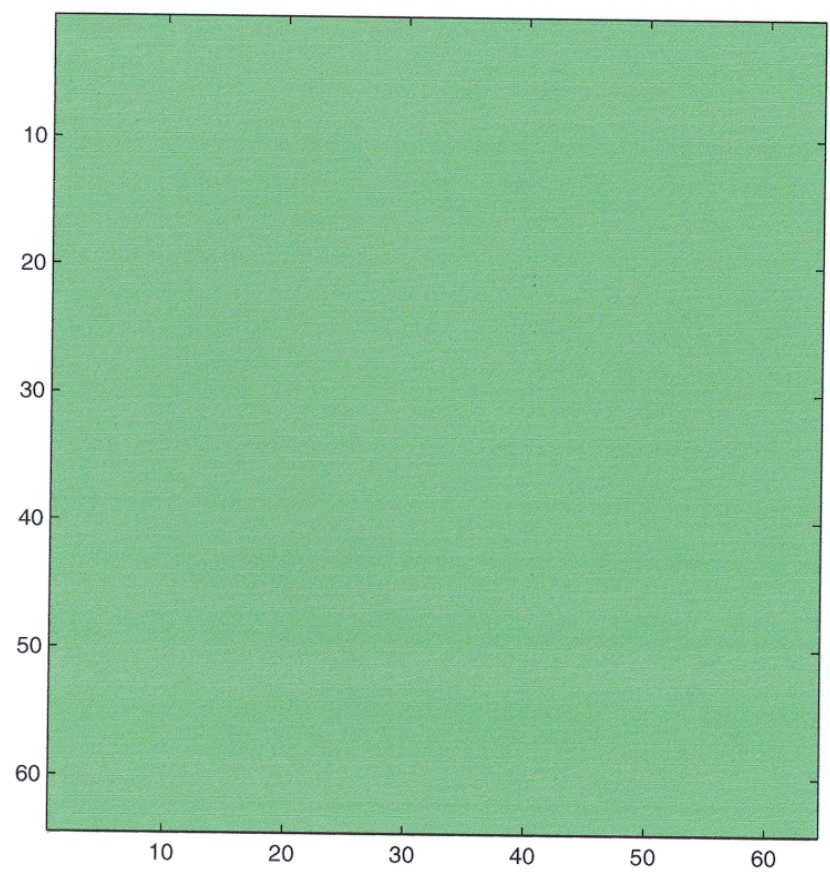

(c)

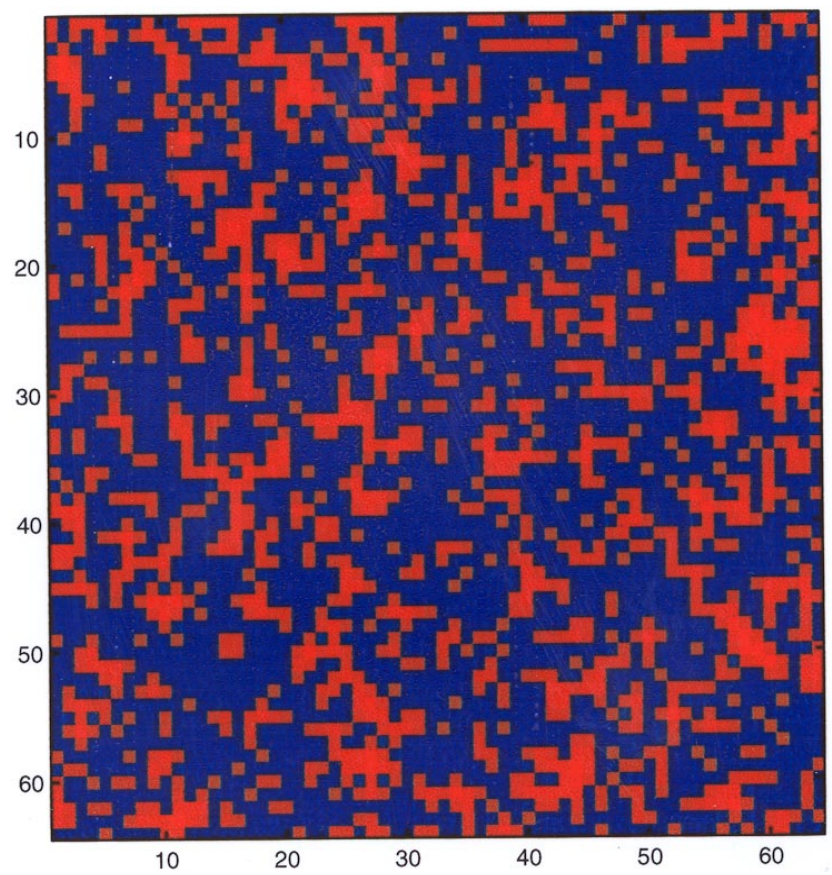

(e)

Fig. 2. (Continued) 
The equilibrium points are given by

$$
\begin{aligned}
V_{1}^{*} & =V_{2}^{*}, \\
V_{1}^{* 3}-\alpha V_{1}^{*}-I^{*} & =0 .
\end{aligned}
$$

Notice that $V_{1}^{*}$ has the same form as that of oneport first-order cells. The Jacobian matrix at an equilibrium point $Q$ is given by

$$
J_{Q}=\left(\begin{array}{cc}
-3 V_{1}^{* 2}+0.5 \alpha-b & -3 V_{1}^{* 2}+1.5 \alpha+b \\
1 & -1
\end{array}\right)
$$

with

$$
\begin{aligned}
& T=-3 V_{1}^{* 2}+0.5 \alpha-b-1, \\
& \Delta=6 V_{1}^{* 2}-2 \alpha .
\end{aligned}
$$

1. From $\operatorname{P} 1\left(\mathbf{Z}_{Q}(s)\right)$ we have

$$
\begin{aligned}
& -3 V_{1}^{* 2}+0.5 \alpha-b-1 \leq 0 \quad \mathbb{A N D} \quad 6 V_{1}^{* 2} \\
& -2 \alpha \geq 0
\end{aligned}
$$

from which we have

$$
\alpha \leq 6 V_{1}^{* 2}+2 b+2 \quad \mathbb{A N D} \quad \alpha \leq 3 V_{1}^{* 2} .
$$

Consequently,

$$
\alpha \leq \begin{cases}6 V_{1}^{* 2}+2 b+2, & \text { if } V_{1}^{* 2} \leq-\frac{2}{3}(b+1), \\ 3 V_{1}^{* 2}, & \text { otherwise }\end{cases}
$$

2. From $\operatorname{P} 2\left(\mathbf{Z}_{Q}(s)\right)$ we have

$$
\begin{gathered}
-3 V_{1}^{* 2}+0.5 \alpha-b \leq 0 \quad \mathbb{A N D} \\
6 V_{1}^{* 2}-2 \alpha \geq 0
\end{gathered}
$$

from which we have

$$
\alpha \leq 6 V_{1}^{* 2}+2 b \quad \mathbb{A N D} \quad \alpha \leq 3 V_{1}^{* 2} .
$$

Consequently,

$$
\alpha \leq \begin{cases}6 V_{1}^{* 2}+2 b, & \text { if } V_{1}^{* 2} \leq-\frac{2}{3} b \\ 3 V_{1}^{* 2}, & \text { otherwise }\end{cases}
$$

3. From P3( $\left.\mathbf{Z}_{Q}(s)\right)$ we have

$$
\begin{gathered}
-3 V_{1}^{* 2}+0.5 \alpha-b=0 \quad \mathbb{A N D} \quad-1=0 \\
-3 V_{1}^{* 2}+1.5 \alpha+b<0,
\end{gathered}
$$

from which we have an empty set.

The parameter region defined by $\mathrm{P} 2\left(\mathbf{Z}_{Q}(s)\right)$ can be a subset of that defined by $\mathrm{P} 1\left(\mathbf{Z}_{Q}(s)\right)$ if we choose a proper parameter $b$. The local passive region is given by

$$
\alpha \leq \begin{cases}6 V_{1}^{* 2}+2 b, & \text { if } V_{1}^{* 2} \leq-\frac{2}{3} b \\ 3 V_{1}^{* 2}, & \text { otherwise }\end{cases}
$$

The edge-of-chaos region is given by

$$
\begin{array}{ll}
6 V_{1}^{* 2}+2 b<\alpha<6 V_{1}^{* 2}+2 b+2 & \mathbb{A N D} \quad V_{1}^{* 2} \\
& \leq-\frac{2}{3}(b+1) .
\end{array}
$$

Comparing with the one-port first-order case where the local passive region is given by $\alpha \leq$ $(3 / 2) V_{1}^{* 2}$, we can see that the local passive region for the one-port second-order case has shrunk and given birth to an edge-of-chaos region. Observe that these conditions are only valid at each individual equilibrium point. The behavior of the entire cell is defined by the combination of conditions considered at all equilibrium points as follows.

\section{Remarks}

From Sec. 2.2.1 we know that we can also find the passive region from $\mathbf{Y}_{Q}(s)$. In fact, $\mathrm{P} 2\left(\mathbf{Y}_{Q}(s)\right)=$ $\mathrm{P} 2\left(\mathbf{Z}_{Q}(s)\right)$ and $\mathrm{P} 1\left(\mathbf{Y}_{Q}(s)\right)$ is much simpler than $\mathrm{P} 1\left(\mathbf{Z}_{Q}(s)\right)$. From $\mathrm{P} 1\left(\mathbf{Y}_{Q}(s)\right)$ we have

$$
a_{22}=-1 \leq 0
$$

which is always satisfied. This means that in this case we do not need to consider $\mathrm{P} 1\left(\mathbf{Z}_{Q}(s)\right)$ as we have already done in Eq. (135). On the other hand, it shows that the results shown in Eq. (135) are redundant. This fact can be seen from Eqs. (135), (138) and (140) where the local passive regions defined in Eq. (140) are exactly the same as those given by $\mathrm{P} 2\left(\mathbf{Z}_{Q}(s)\right)$; namely, Eq. (138).

\subsection{Passive parameter regions}

1. When $\mathfrak{D}>0$, the local passive regions are given by 


$$
\begin{aligned}
& \frac{I^{* 2}}{4}-\frac{\alpha^{3}}{27}>0, \\
& \alpha \leq \begin{cases}6\left[\left(\frac{I^{*}}{2}+\sqrt{\mathfrak{D}}\right)^{1 / 3}+\left(\frac{I^{*}}{2}-\sqrt{\mathfrak{D}}\right)^{1 / 3}\right]^{2}+2 b, & \text { if }\left[\left(\frac{I^{*}}{2}+\sqrt{\mathfrak{D}}\right)^{1 / 3}+\left(\frac{I^{*}}{2}-\sqrt{\mathfrak{D}}\right)^{1 / 3}\right]^{2} \leq-\frac{2}{3} b, \\
3\left[\left(\frac{I^{*}}{2}+\sqrt{\mathfrak{D}}\right)^{1 / 3}+\left(\frac{I^{*}}{2}-\sqrt{\mathfrak{D}}\right)^{1 / 3}\right]^{2}, & \text { otherwise }\end{cases}
\end{aligned}
$$

which is the intersection of the following two parameter regions:

$$
\begin{gathered}
\frac{I^{* 2}}{4}-\frac{\alpha^{3}}{27}>0 \quad \mathbb{A N D} \quad \alpha \leq 6\left[\left(\frac{I^{*}}{2}+\sqrt{\mathfrak{D}}\right)^{1 / 3}+\left(\frac{I^{*}}{2}-\sqrt{\mathfrak{D}}\right)^{1 / 3}\right]^{2}+2 b \quad \mathbb{A N D} \\
{\left[\left(\frac{I^{*}}{2}+\sqrt{\mathfrak{D}}\right)^{1 / 3}+\left(\frac{I^{*}}{2}-\sqrt{\mathfrak{D}}\right)^{1 / 3}\right]^{2} \leq-\frac{2}{3} b,}
\end{gathered}
$$

and

$$
\frac{I^{* 2}}{4}-\frac{\alpha^{3}}{27}>0 \quad \mathbb{A N D} \quad\left[\left(\frac{I^{*}}{2}+\sqrt{\mathfrak{D}}\right)^{1 / 3}+\left(\frac{I^{*}}{2}-\sqrt{\mathfrak{D}}\right)^{1 / 3}\right]^{2}>-\frac{2}{3} b .
$$

2. When $\mathfrak{D}=0$, since there are two equilibrium points, the local passive region is the intersection of the local passive parameter regions for both equilibrium points and are given by

$$
\begin{aligned}
& \frac{I^{* 2}}{4}-\frac{\alpha^{3}}{27}=0 \quad \mathbb{A N D} \quad \alpha \leq\left\{\begin{array}{l}
24\left(\frac{I^{*}}{2}\right)^{2 / 3}+2 b, \quad \text { if } 4\left(\frac{I^{*}}{2}\right)^{2 / 3} \leq-\frac{2}{3} b, \\
12\left(\frac{I^{*}}{2}\right)^{2 / 3}, \quad \text { otherwise }
\end{array}\right. \\
& \alpha \leq \begin{cases}6\left(\frac{I^{*}}{2}\right)^{2 / 3}+2 b, & \text { if }\left(\frac{I^{*}}{2}\right)^{2 / 3} \leq-\frac{2}{3} b, \\
3\left(\frac{I^{*}}{2}\right)^{2 / 3}, & \text { otherwise }\end{cases}
\end{aligned}
$$

from which we have

$$
\alpha \geq 0 \quad \mathbb{A N D} \quad \alpha \leq\left\{\begin{array}{cl}
8 \alpha+2 b, & \text { if } \alpha \leq-0.5 b, \\
4 \alpha, & \text { otherwise }
\end{array} \quad \mathbb{N} \mathbb{N D} \quad \alpha \leq\left\{\begin{array}{cl}
2 \alpha+2 b, & \text { if } \alpha \leq-0.5 b \\
\alpha, & \text { otherwise }
\end{array}\right.\right.
$$

If $b \geq 0$ then the local passive region is a point $\alpha=0, I^{*}=0$. If $b<0$ then the local passive region is given by two points at

$$
\left(\alpha=-0.5 b, I^{*}=2(-0.5 b / 3)^{3 / 2}\right),\left(\alpha=-0.5 b, I^{*}=-2(-0.5 b / 3)^{3 / 2}\right) .
$$

3. When $\mathfrak{D}<0$, since there are three equilibrium points, the local passive regions are given by the intersection of the local passive parameter regions at these equilibrium points as

$$
\frac{I^{* 2}}{4}-\frac{\alpha^{3}}{27}<0 \quad \mathbb{A N D} \quad \alpha \leq\left\{\begin{array}{ll}
6(2 \sqrt{|\alpha| / 3} \cos (\psi / 3))^{2}+2 b, & \text { if }(2 \sqrt{|\alpha| / 3} \cos (\psi / 3))^{2} \leq-\frac{2}{3} b, \\
3(2 \sqrt{|\alpha| / 3} \cos (\psi / 3))^{2}, & \text { otherwise }
\end{array} \quad \mathbb{A N D}\right.
$$




$$
\begin{aligned}
& \alpha \leq \begin{cases}6(-2 \sqrt{|\alpha| / 3} \cos (\psi / 3-\pi / 3))^{2}+2 b, & \text { if }(-2 \sqrt{|\alpha| / 3} \cos (\psi / 3-\pi / 3))^{2} \leq-\frac{2}{3} b, \\
3(-2 \sqrt{|\alpha| / 3} \cos (\psi / 3-\pi / 3))^{2}, & \text { otherwise }\end{cases} \\
& \alpha \leq \begin{cases}6(-2 \sqrt{|\alpha| / 3} \cos (\psi / 3+\pi / 3))^{2}+2 b, & \text { if }(-2 \sqrt{|\alpha| / 3} \cos (\psi / 3+\pi / 3))^{2} \leq-\frac{2}{3} b, \\
3(-2 \sqrt{|\alpha| / 3} \cos (\psi / 3+\pi / 3))^{2}, & \text { otherwise. }\end{cases}
\end{aligned}
$$

This set of conditions give an empty set.

\subsection{Edge-of-chaos parameter regions}

From the analysis of the local passive parameter region presented Sec. 5.1 we know that the edge-of-chaos region can only be found when $\mathfrak{D}>0$. The edge-of-chaos region is then given by the following conditions:

$$
\begin{array}{rl}
b<0 & \mathbb{A N D} \quad \frac{I^{* 2}}{4}-\frac{\alpha^{3}}{27}>0 \quad \mathbb{A N D} \quad 6\left[\left(\frac{I^{*}}{2}+\sqrt{\mathfrak{D}}\right)^{1 / 3}+\left(\frac{I^{*}}{2}-\sqrt{\mathfrak{D}}\right)^{1 / 3}\right]^{2}+2 b \\
& <\alpha<6\left[\left(\frac{I^{*}}{2}+\sqrt{\mathfrak{D}}\right)^{1 / 3}+\left(\frac{I^{*}}{2}-\sqrt{\mathfrak{D}}\right)^{1 / 3}\right]^{2}+2 b+2 \quad \mathbb{A N D}\left[\left(\frac{I^{*}}{2}+\sqrt{\mathfrak{D}}\right)^{1 / 3}+\left(\frac{I^{*}}{2}-\sqrt{\mathfrak{D}}\right)^{1 / 3}\right]^{2} \\
& \leq-\frac{2}{3}(b+1)
\end{array}
$$

\subsection{Illustrative examples}

In Fig. 3 the locally passive, edge-of-chaos and locally active parameter regions are shown in the $I^{*}-\alpha$ plane. The locally passive regions and restricted locally passive regions are shown in blue and cyan, respectively. The edge-of-chaos regions and the locally active unstable regions are shown in red and green, respectively. When $b \geq 0$, the edge-of-chaos parameter region is empty and the $I^{*}-\alpha$ plane is the same as that shown in Fig. 1. When $0<b \leq-1$, the upper boundary of the edge-of-chaos region is given by the curve $\mathfrak{D}=0$. In Fig. 3 we show the edge-of-chaos regions with $b=-1,-10$ and -20 , respectively. As we can see from Fig. 3, when $b$ decreases, the edge-of-chaos region changes from the shape of a needle to the shape of a "two-tooth" fork. Also, as $b$ decreases, the length of both fork teeth and the distance between them increase. As we will see later in this section, this shape bifurcation of the edge-of-chaos regions corresponds to a bifurcation of pattern formation in the corresponding CNNs.

We will now present our simulation results of an $N \times N$ CNN described by the following equations:

$$
\begin{aligned}
\dot{V}_{1}(i, j)= & -V_{1}^{3}(i, j)+(0.5 \alpha-b) V_{1}(i, j)-V_{2}^{3}(i, j)+(1.5 \alpha+b) V_{2}(i, j)+D_{1}\left[V_{1}(i-1, j)\right. \\
& \left.+V_{1}(i, j-1)+V_{1}(i, j+1)+V_{1}(i+1, j)-4 V_{1}(i, j)\right] \\
\dot{V}_{2}(i, j)= & V_{1}(i, j)-V_{2}(i, j), 1 \leq i \leq N, 1 \leq j \leq N .
\end{aligned}
$$

The simulation results with parameters $\alpha=$ $-3, b=-1$ and $D_{1}=1$ are shown in Fig. 4. As shown in Figs. 3(a)-3(c), this CNN is locally passive with these parameters. Figures $4(\mathrm{a})$ and $4(\mathrm{~b})$ show the initial conditions of $V_{1}(i, j)$ and $V_{2}(i, j)$, respectively. Figures $4(\mathrm{c})$ and $4(\mathrm{~d})$ show $V_{1}(i, j)$ and $V_{2}(i, j)$ at $t=1$, respectively. Figures $4(\mathrm{e})$ and $4(\mathrm{f})$ show $V_{1}(i, j)$ and $V_{2}(i, j)$ at $t=4$, respectively. Observe that this CNN converges to a homogeneous pattern. 
(a)

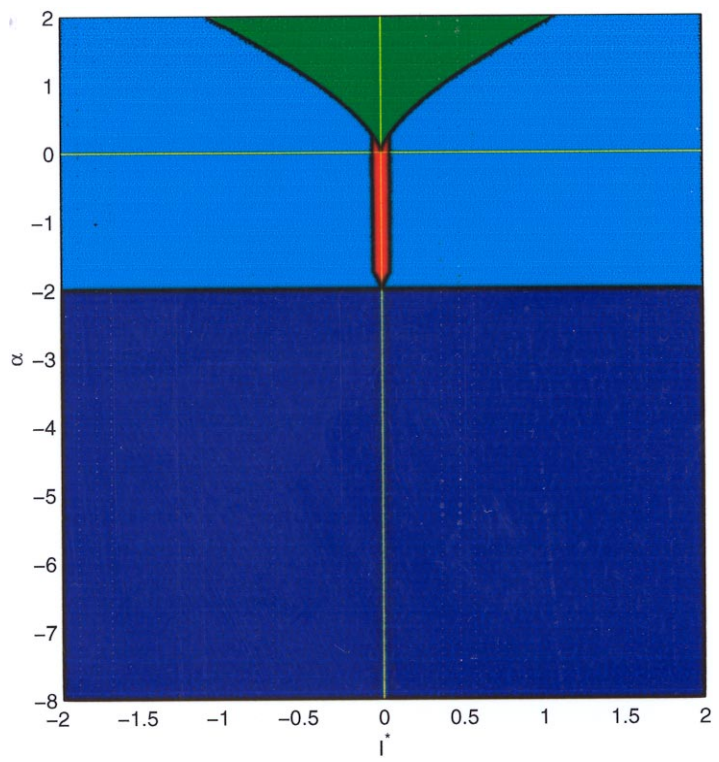

(b)

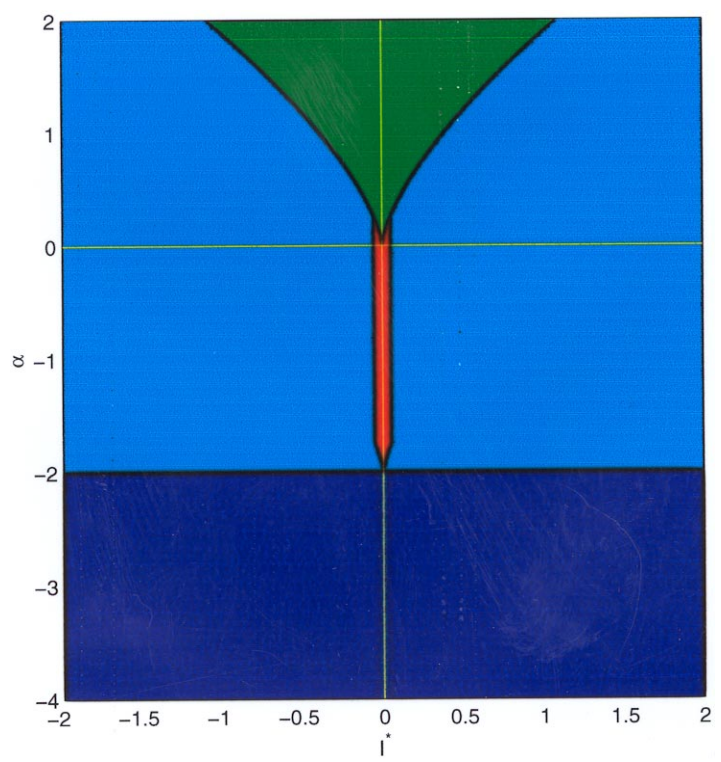

(c)

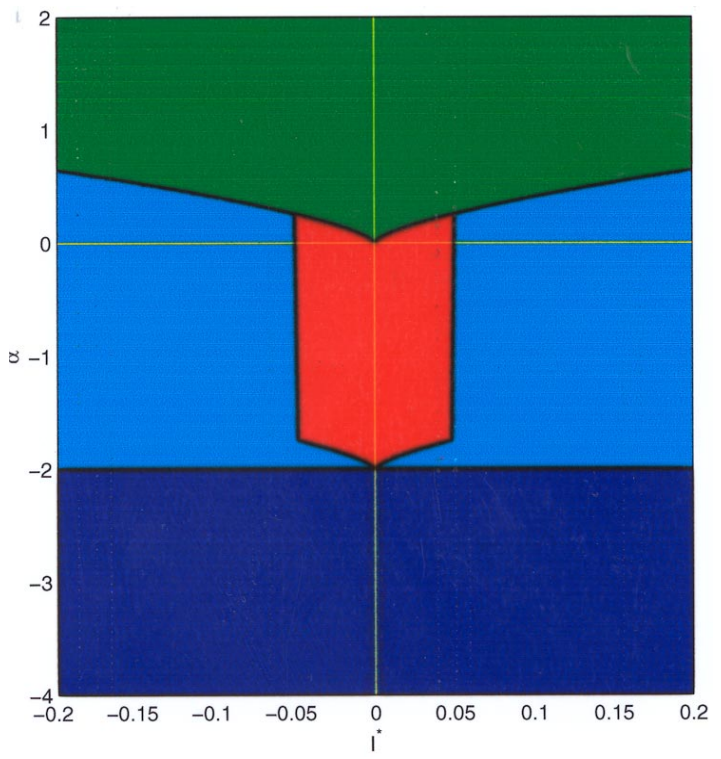

The simulation results with parameters $\alpha=1$, $b=-1$ and $D_{1}=1$ are shown in Fig. 5. As shown in Fig. 3(a)-3(c), this CNN is locally active with these parameters. The initial conditions are the same as those in Figs. 4(a) and 4(b). Figures 5(a) and $5(\mathrm{~b})$ show $V_{1}(i, j)$ and $V_{2}(i, j)$ at $t=1$, respectively. Figures $5(\mathrm{c})$ and $5(\mathrm{~d})$ show $V_{1}(i, j)$ and $V_{2}(i, j)$ at $t=10$, respectively. Figures $5(\mathrm{e})$ and $5(\mathrm{f})$ show $V_{1}(i, j)$ and $V_{2}(i, j)$ at $t=500$, respectively. Figures $5(\mathrm{~g})$ and $5(\mathrm{~h})$ show $V_{1}(i, j)$ and $V_{2}(i, j)$ at $t=1000$, respectively. Observe that the final states converge to a static pattern where $V_{1}(i, j)=V_{2}(i, j)$. The mechanism leading to this kind of pattern can be understood intuitively as follows. The cells push the states away from a homogeneous pattern because $I^{*}=0$ is locally active in this case. However, as the coupling current $I^{*}$ generated by the evolving pattern becomes large enough such that $\left|I^{*}\right|$ enters the restricted locally passive regions, this evolving pattern will eventually be frozen in the locally passive regions. In other words, this CNN will settle down at an equilibrium point in the restricted locally passive regions.

The simulation results with parameters $\alpha=$ $-1, b=-1$ and $D_{1}=1$ are shown in Fig. 6 . As shown in Figs. 3(a)-3(c), this CNN is operating in the edge-of-chaos parameter region. The initial conditions for $V_{1}(i, j)$ and $V_{2}(i, j)$ are the same as those shown in Figs. 4(a) and 4(b). Figures 6(a) and $6(\mathrm{~b})$ show $V_{1}(i, j)$ and $V_{2}(i, j)$ at $t=1$, respectively. Figures $6(\mathrm{c})$ and $6(\mathrm{~d})$ show $V_{1}(i, j)$ and $V_{2}(i, j)$ at $t=4$, respectively. Figures $6(\mathrm{e})$ and $6(\mathrm{f})$ show $V_{1}(i, j)$ and $V_{2}(i, j)$ at $t=10$, respectively. Figures $6(\mathrm{~g})-6(\mathrm{i})$ show $V_{1}(i, j)$ at $t=20,40$ and 80 , respectively. Observe that this CNN converges to relatively "smooth" patterns. This is because the edge-of-chaos parameter region as shown in Figs. 3(a)-3(c) is very near the zero cell bias; namely, $I^{*} \ll 1$. In this case, the pattern is

Fig. 3. Parameter regions for one-port second-order cells. The locally passive regions and restricted locally passive regions are shown in blue and cyan, respectively. The edge-ofchaos regions and locally active unstable regions are shown in red and green, respectively. (a) $b=-1$. (b) $b=-1$, enlargement of (a). (c) $b=-1$, enlargement of (b). (d) $b=-10$. (e) $b=-10$, enlargement of (d). (f) $b=-10$, enlargement of (e). (g) $b=-20$. (h) $b=-20$, enlargement of (g). (i) $b=-20$, enlargement of (h). 
(d)

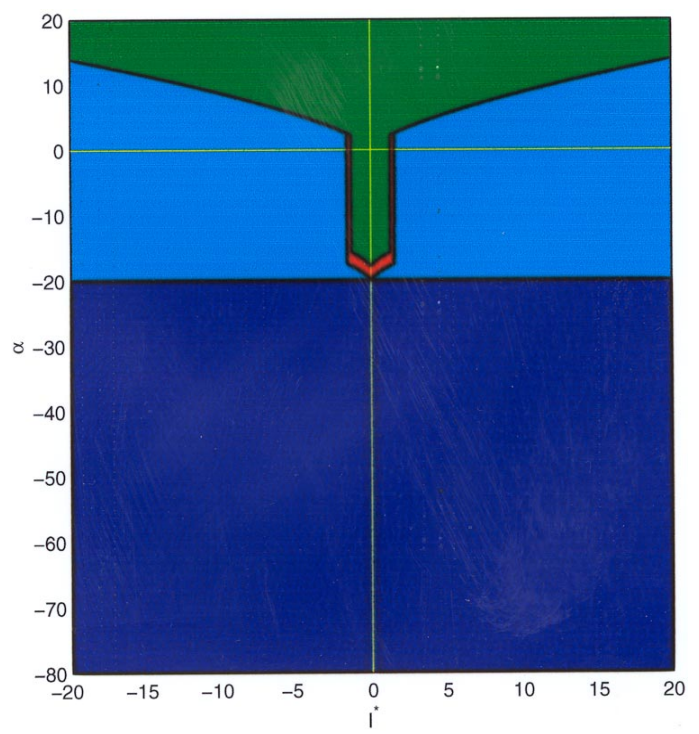

(e)

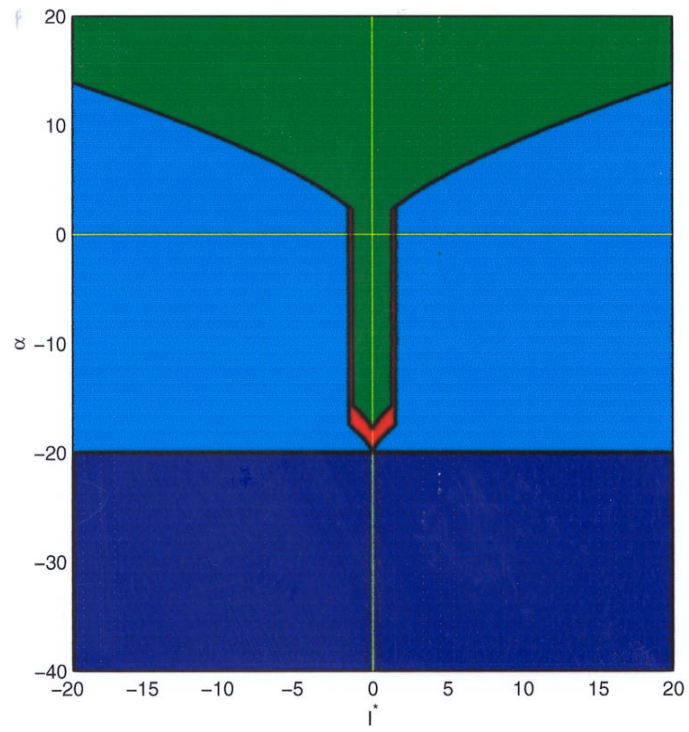

(f)

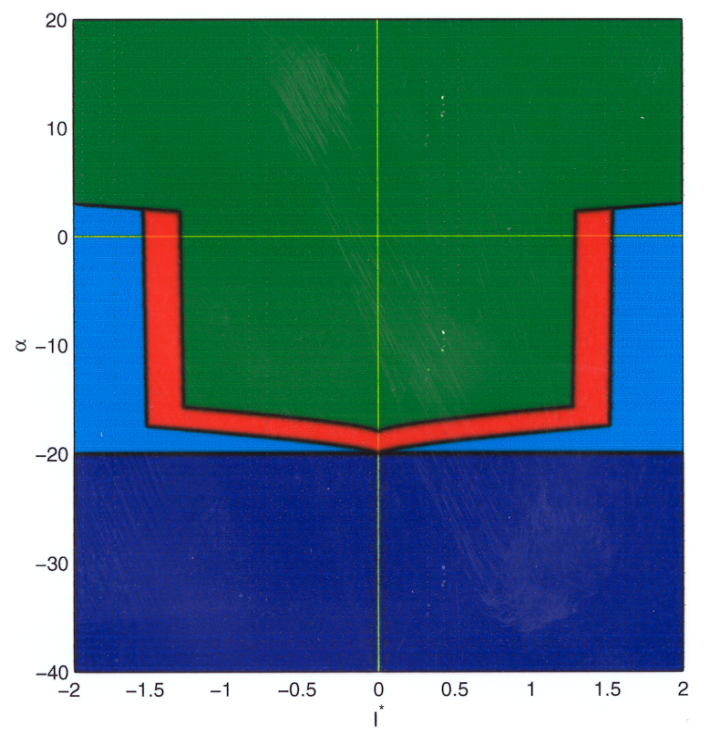

(g)

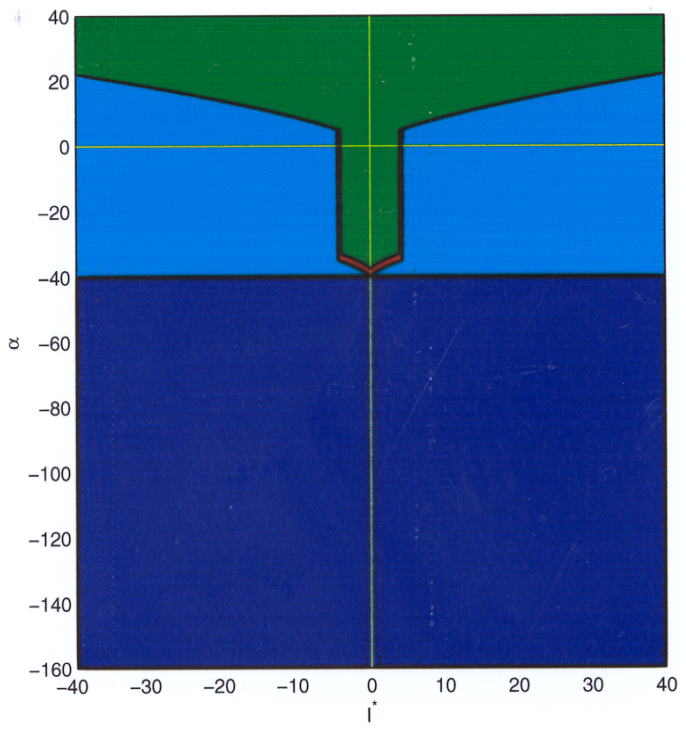

(h)

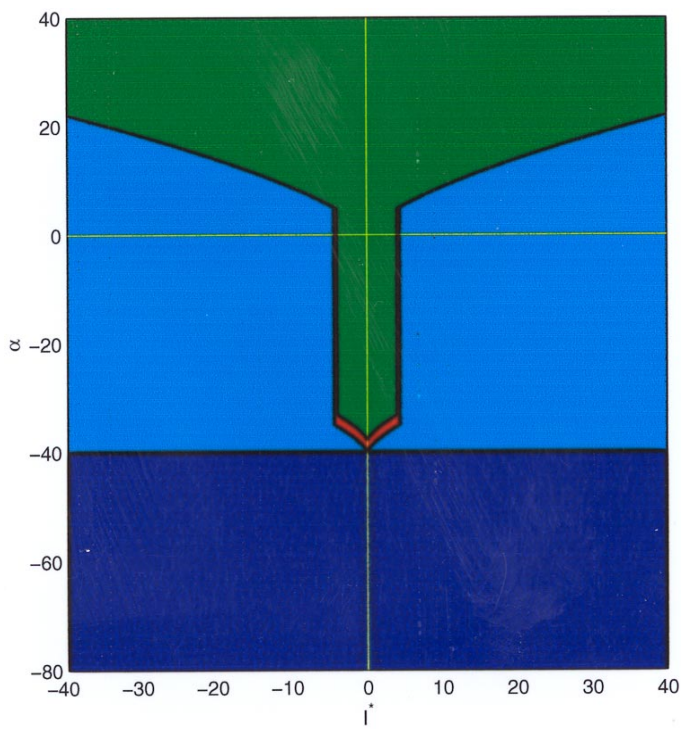

(i)

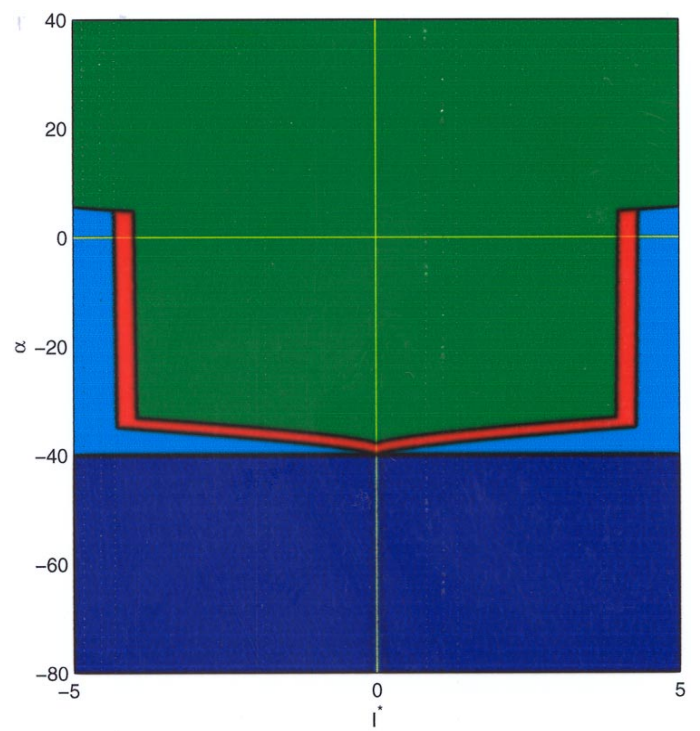

Fig. 3 (Continued) 


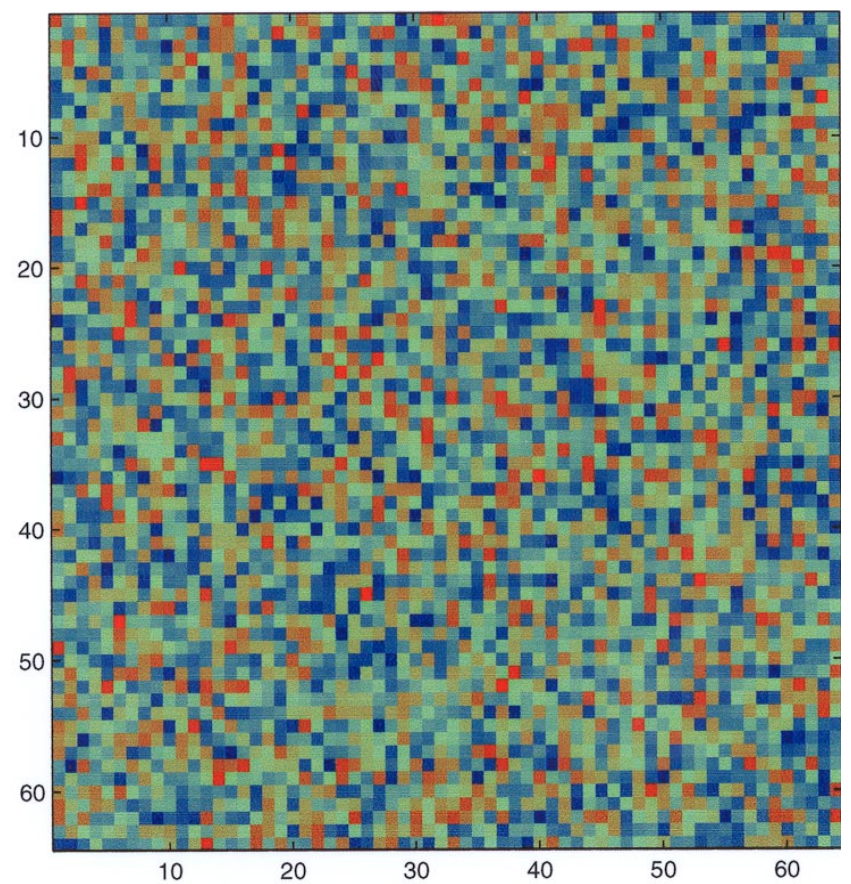

(a)

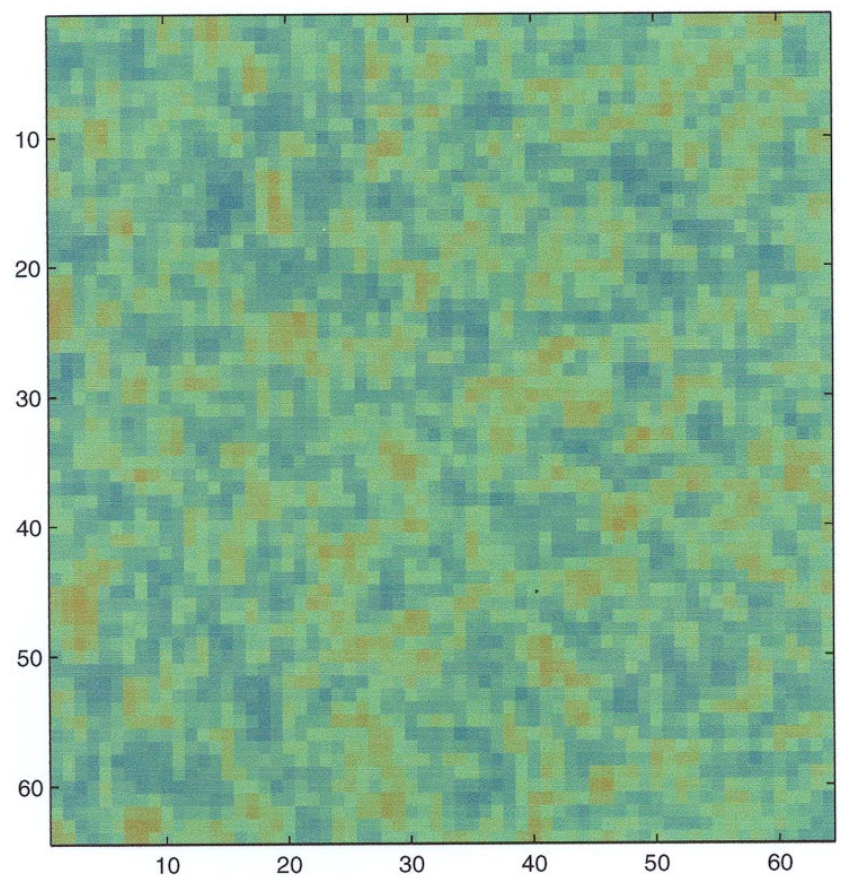

(c)

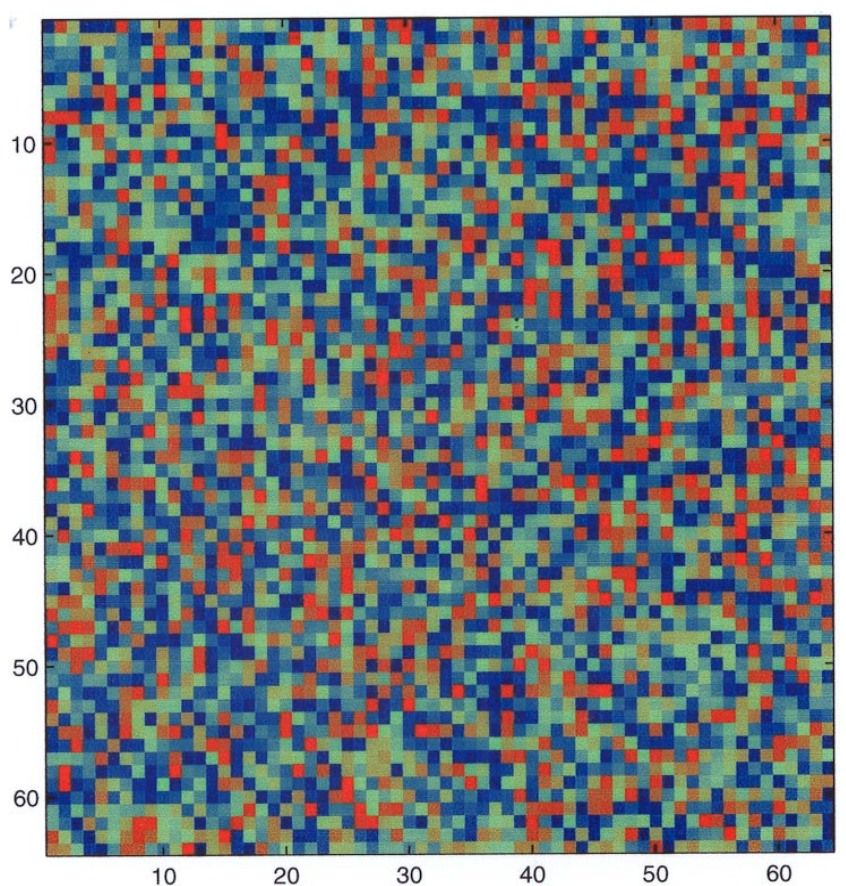

(b)

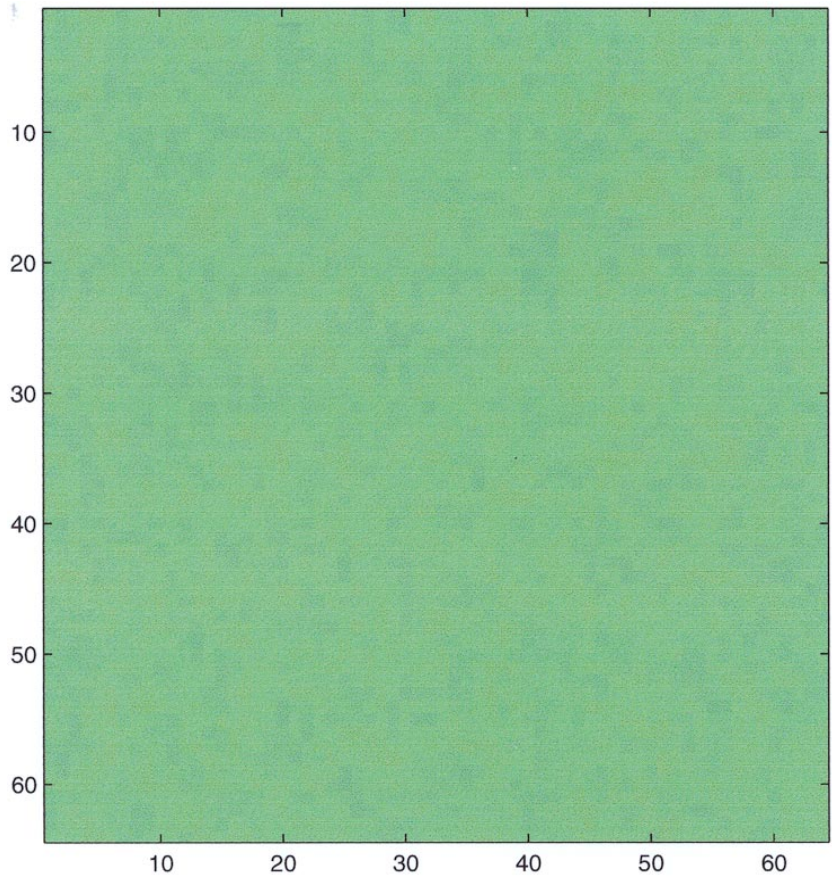

(d)

Fig. 4. Simulation results of CNN made of one-port second-order cells. The parameters are given by $\alpha=-3, b=-1$ and $D_{1}=1$. The cells are locally passive. (a) Initial condition for $V_{1}(i, j)$. (b) Initial condition for $V_{2}(i, j)$. (c) $V_{1}(i, j)$ at $t=1$. (d) $V_{2}(i, j)$ at $t=1$. (e) $V_{1}(i, j)$ at $t=4$. (f) $V_{2}(i, j)$ at $t=4$. 


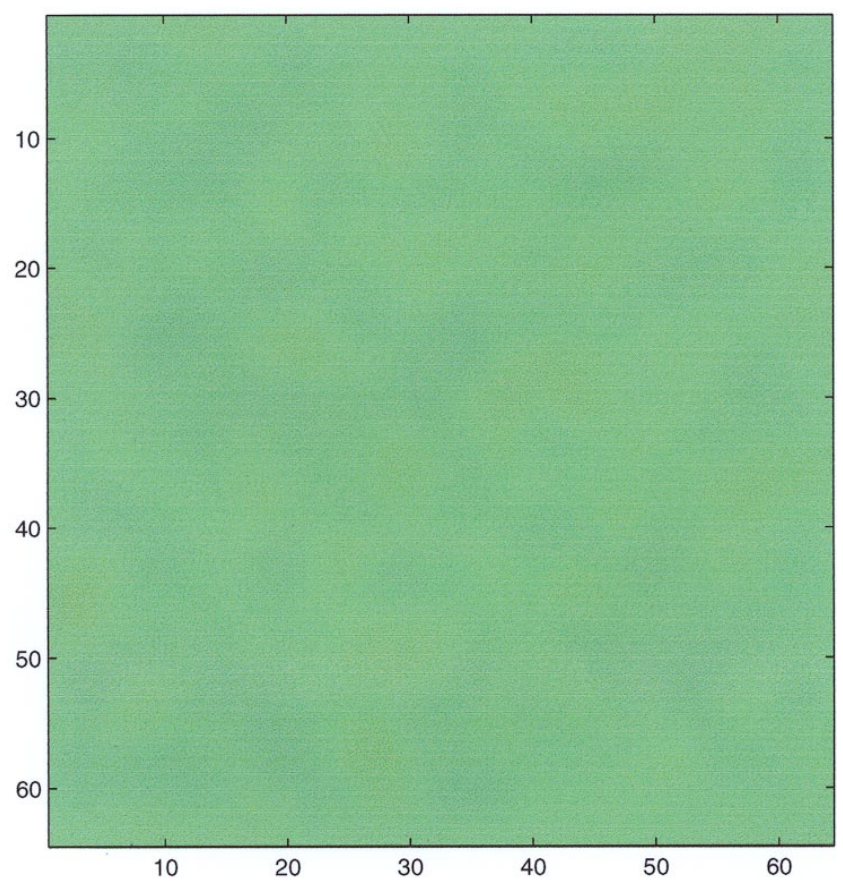

(e)

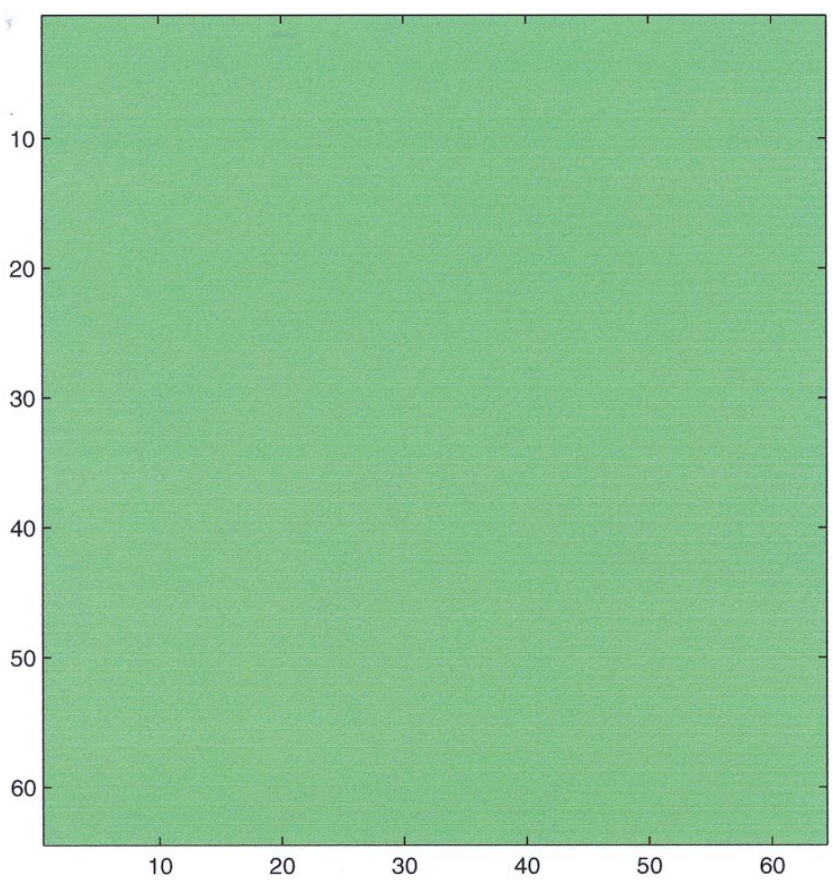

(f)

Fig. 4. (Continued)

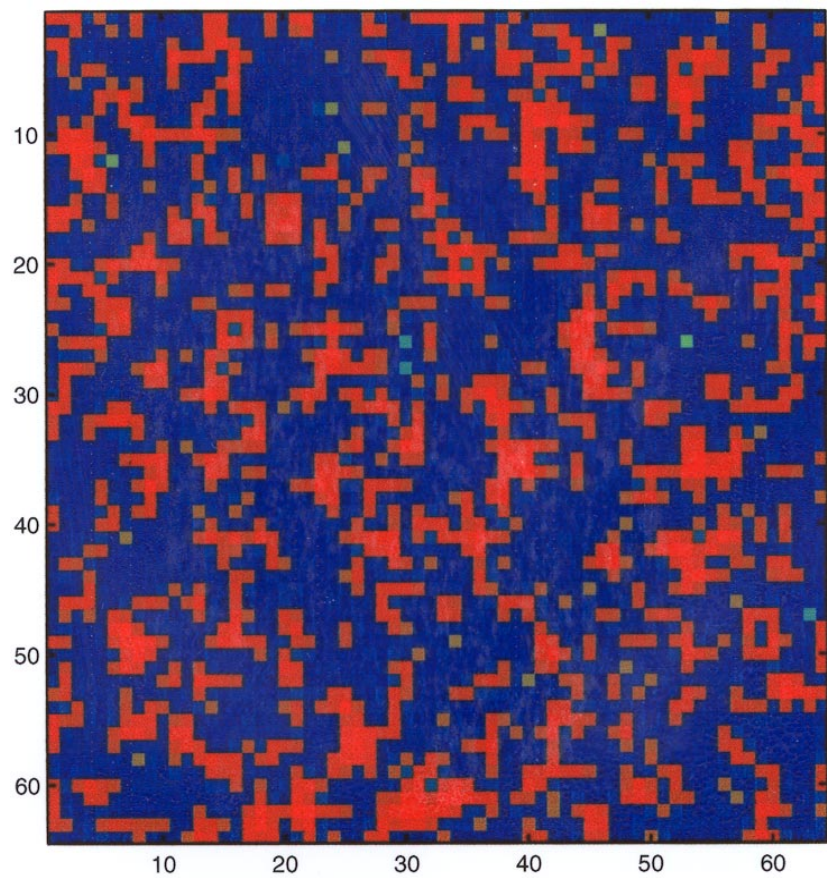

(a)

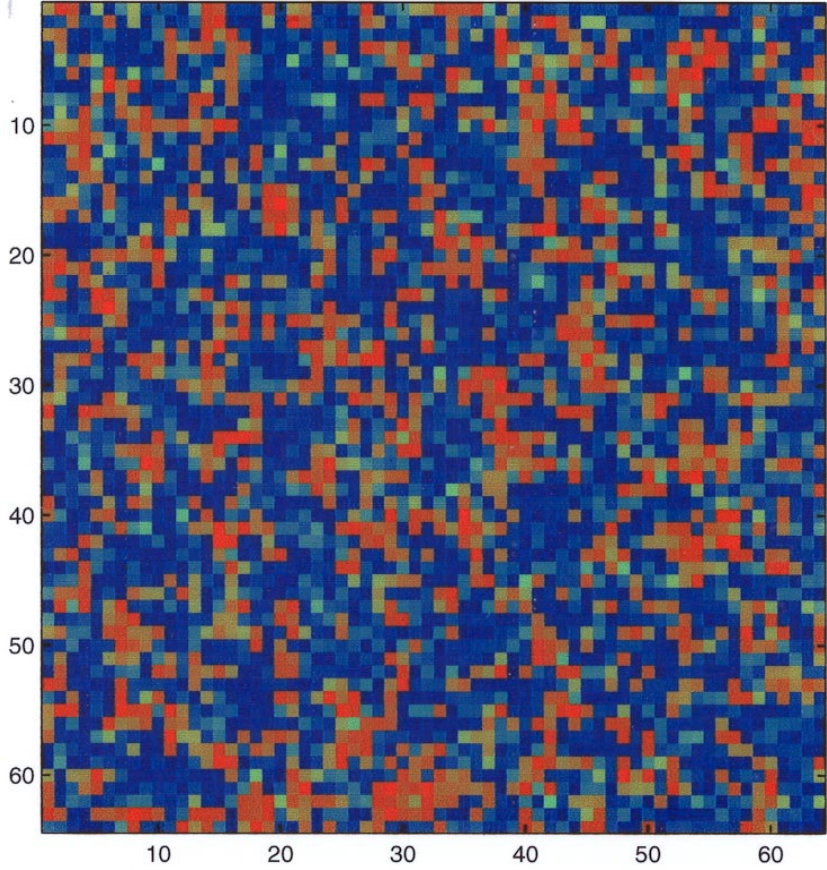

(b)

Fig. 5. Simulation results of CNN made of one-port second-order cells. The parameters are given by $\alpha=1, b=-1$ and $D_{1}=1$. The cells are locally active. (a) $V_{1}(i, j)$ at $t=1$. (b) $V_{2}(i, j)$ at $t=1$. (c) $V_{1}(i, j)$ at $t=10$. (d) $V_{2}(i, j)$ at $t=10$. (e) $V_{1}(i, j)$ at $t=500$. (f) $V_{2}(i, j)$ at $t=500$. (g) $V_{1}(i, j)$ at $t=1000$. (h) $V_{2}(i, j)$ at $t=1000$. 


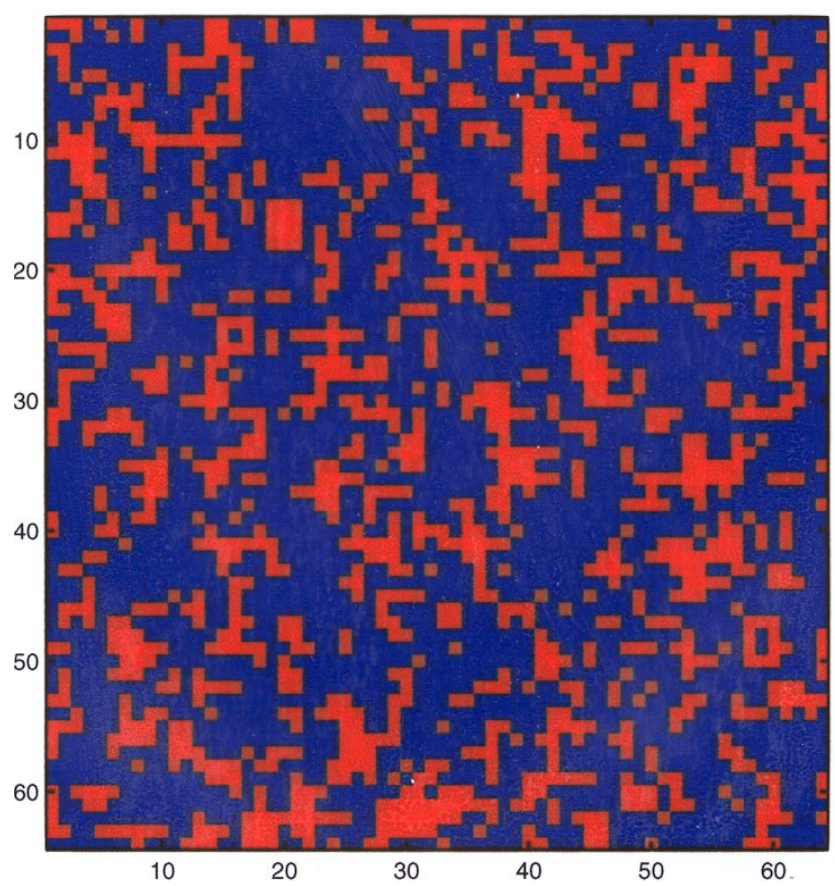

(c)

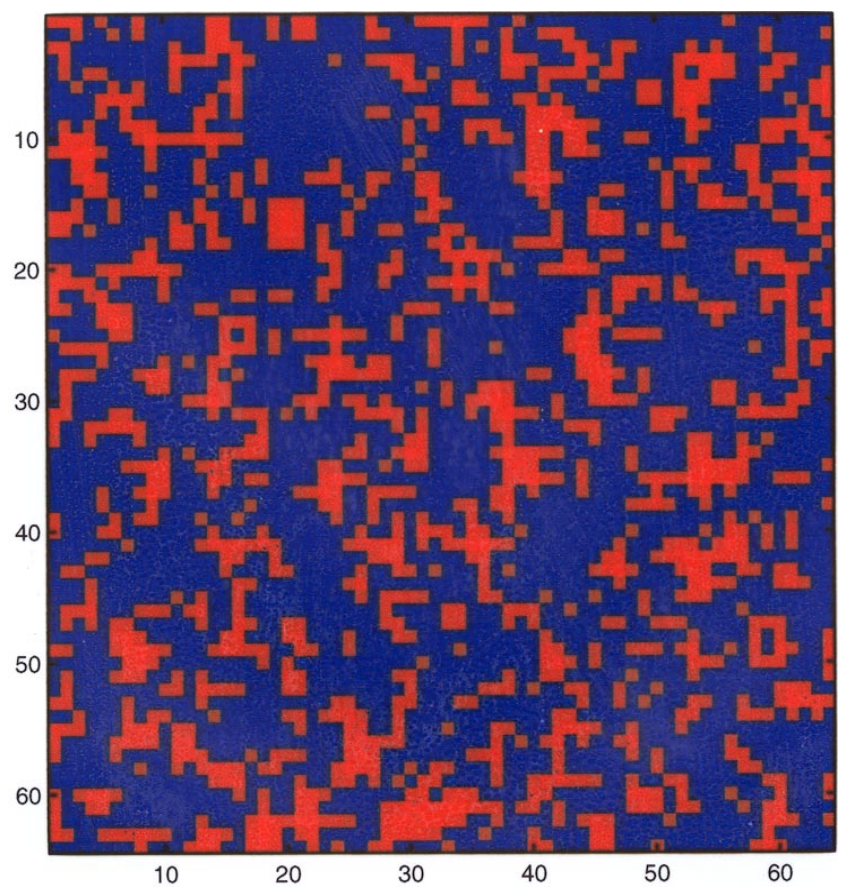

(e)

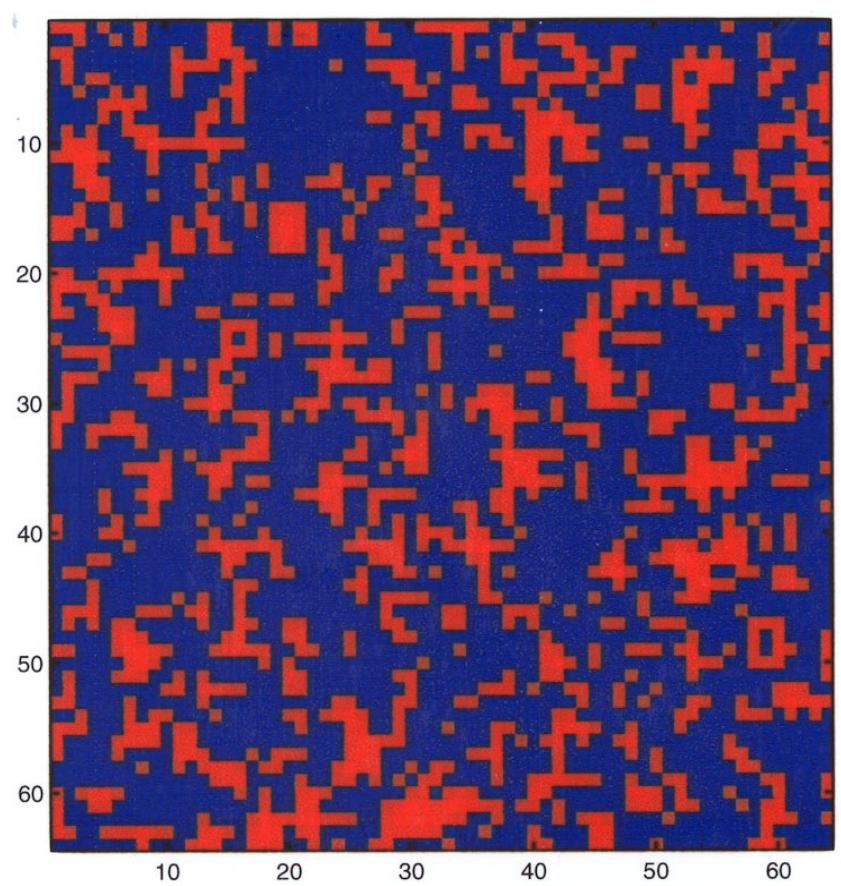

(d)

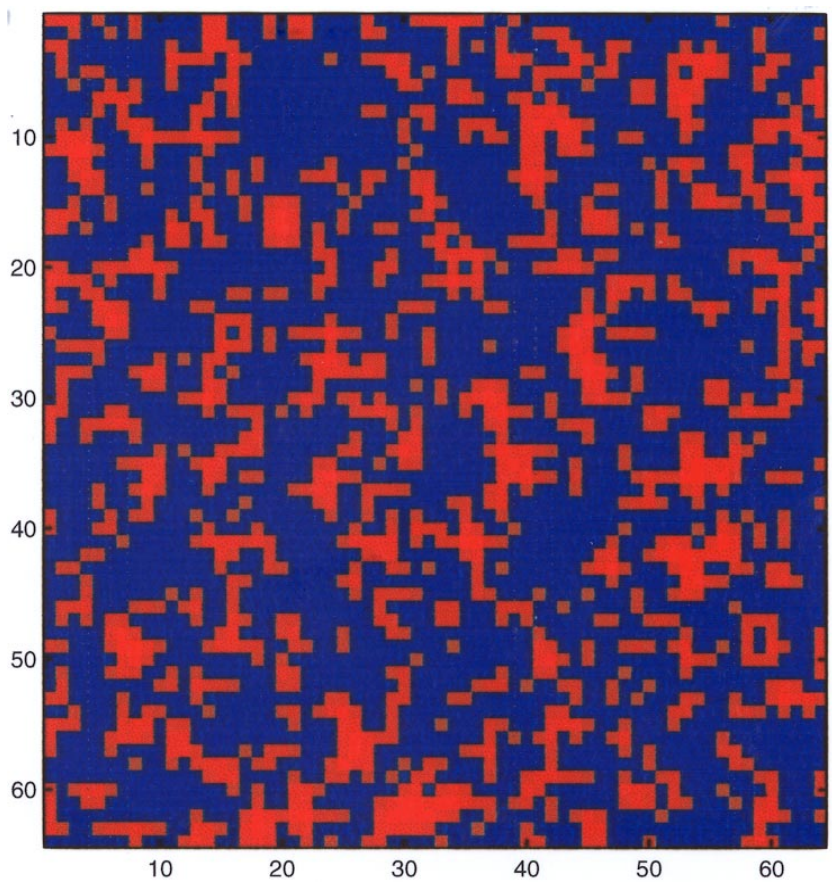

(f)

Fig. 5. (Continued) 

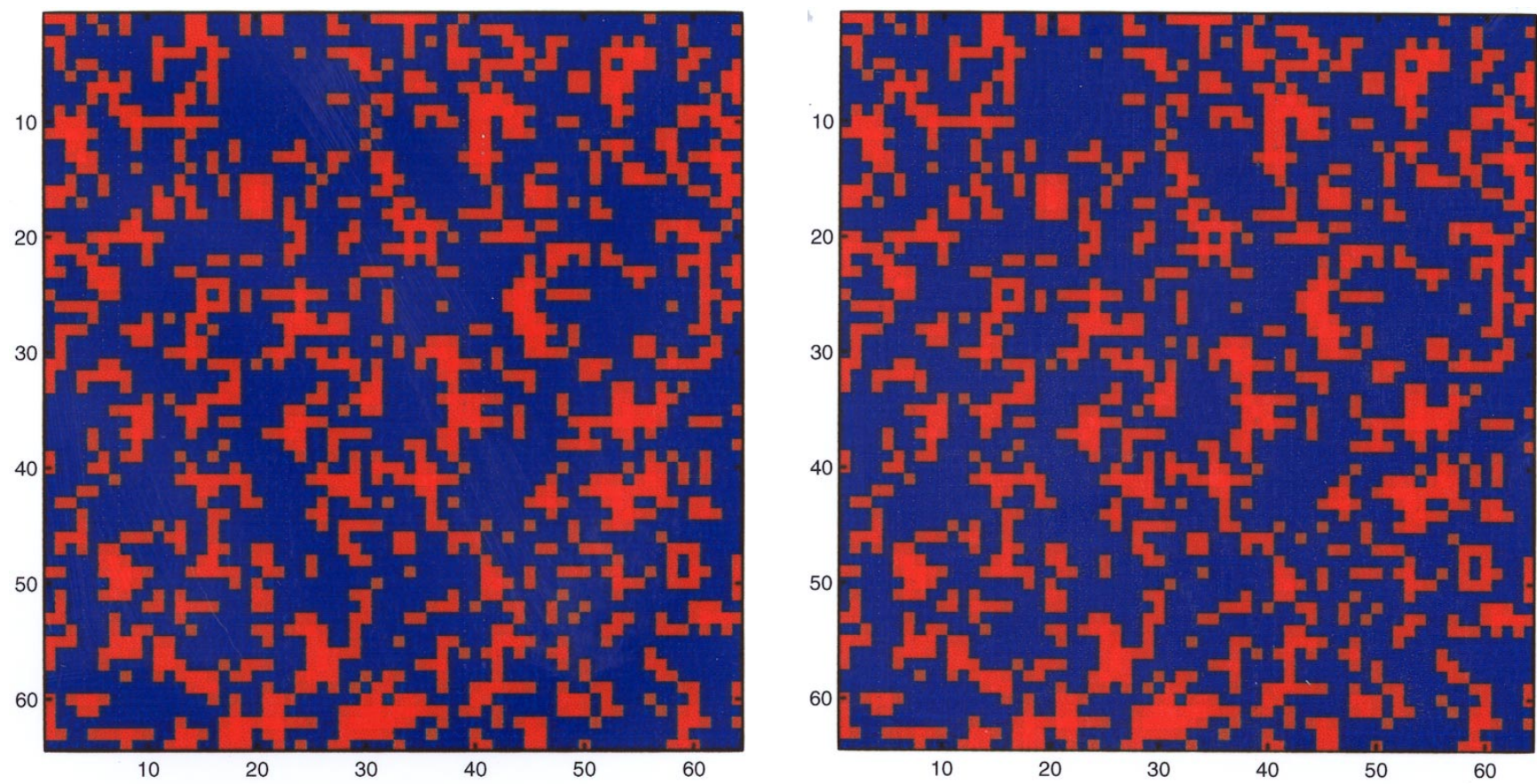

(g)

(h)

Fig. 5. (Continued)

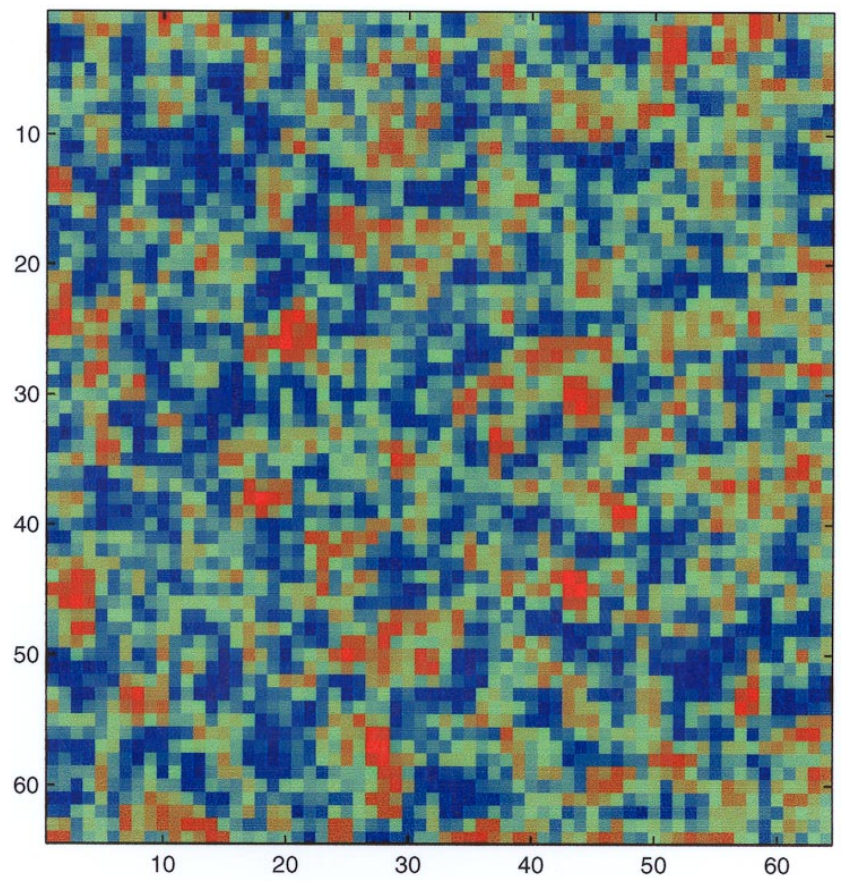

(a)

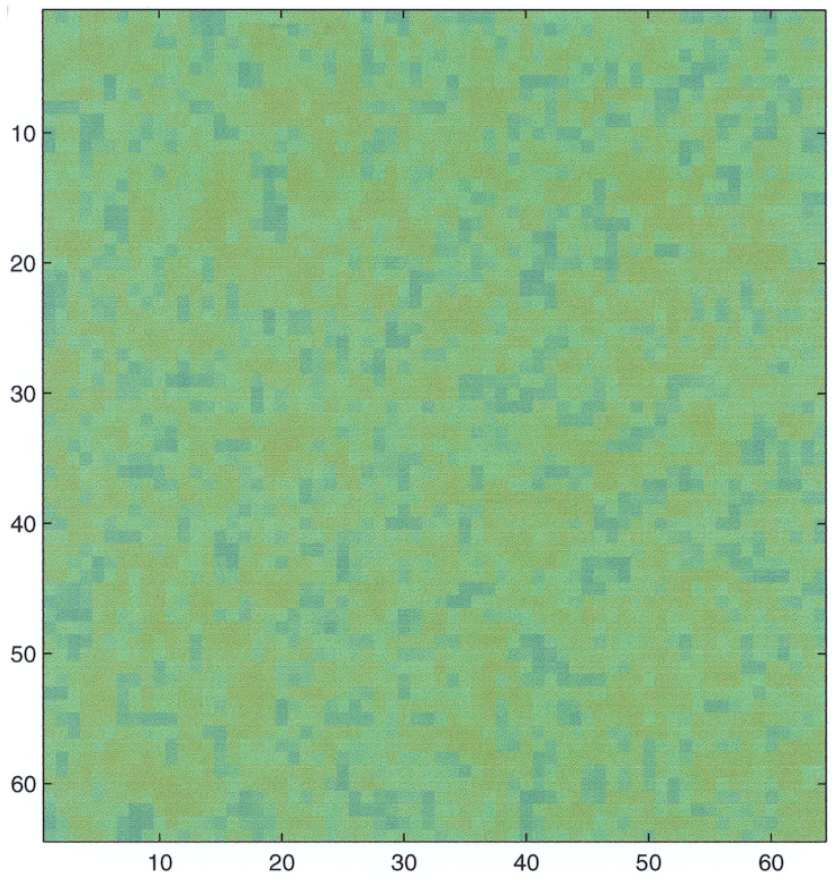

(b)

Fig. 6. Simulation results of CNN made of one-port second-order cells. The parameters are given by $\alpha=-1, b=-1$ and $D_{1}=1$. The cells are in edge-of-chaos region. (a) $V_{1}(i, j)$ at $t=1$. (b) $V_{2}(i, j)$ at $t=1$. (c) $V_{1}(i, j)$ at $t=4$. (d) $V_{2}(i, j)$ at $t=4$. (e) $V_{1}(i, j)$ at $t=10$. (f) $V_{2}(i, j)$ at $t=10$. (g) $V_{1}(i, j)$ at $t=20$. (h) $V_{1}(i, j)$ at $t=40$. (i) $V_{1}(i, j)$ at $t=80$. (j) $V_{1}(i, j)$ at $t=100$. (k) $V_{2}(i, j)$ at $t=100$. (l) $V_{1}(i, j)$ at $t=200$. (m) $V_{2}(i, j)$ at $t=200$. (n) $V_{1}(i, j)$ at $t=300$. (o) $V_{2}(i, j)$ at $t=300$. (p) $V_{1}(i, j)$ at $t=600$. (q) $V_{2}(i, j)$ at $t=600$. (r) $V_{1}(i, j)$ at $t=1000$. (s) $V_{2}(i, j)$ at $t=1000$. (t) $V_{1}(i, j)$ at $t=2000$. (u) $V_{2}(i, j)$ at $t=2000$. 


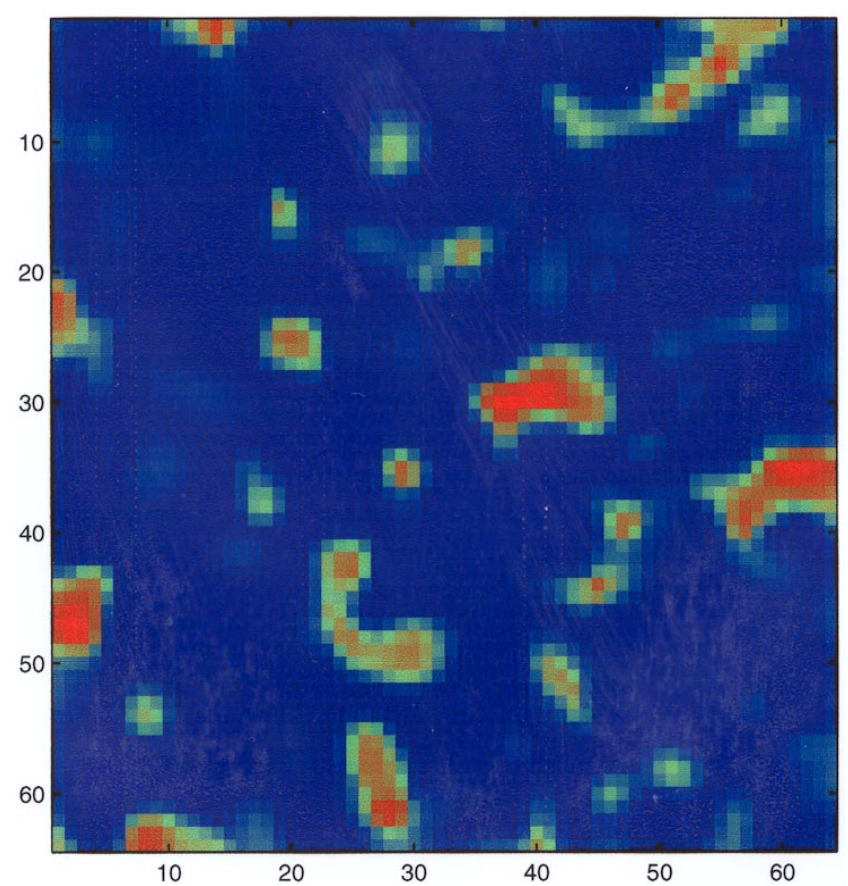

(c)

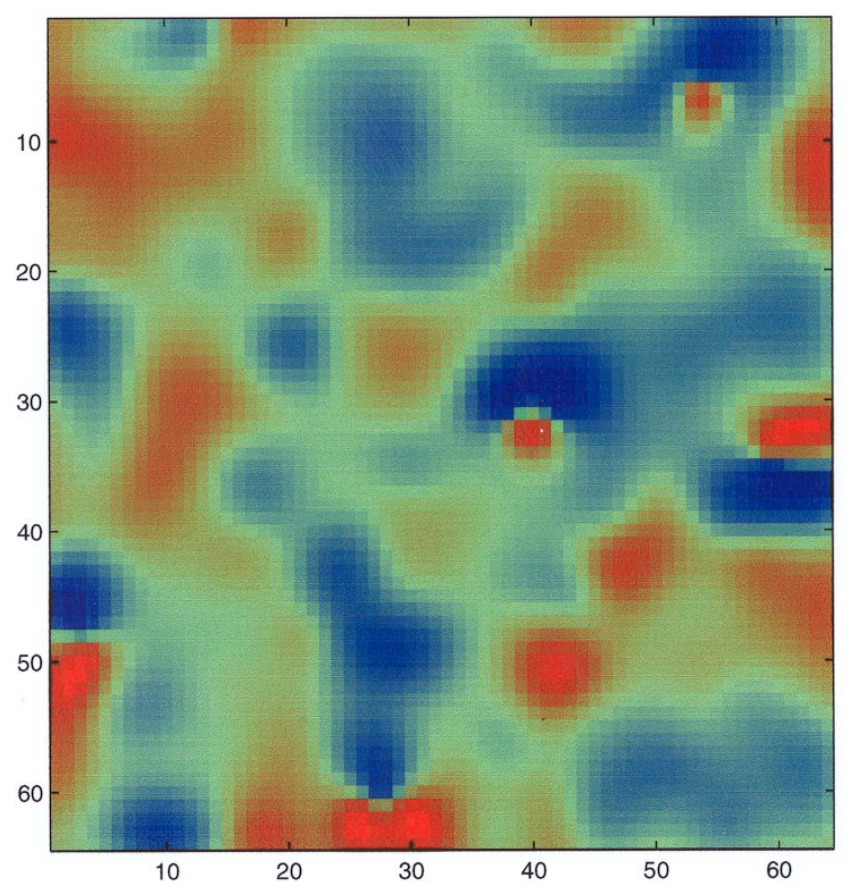

(e)

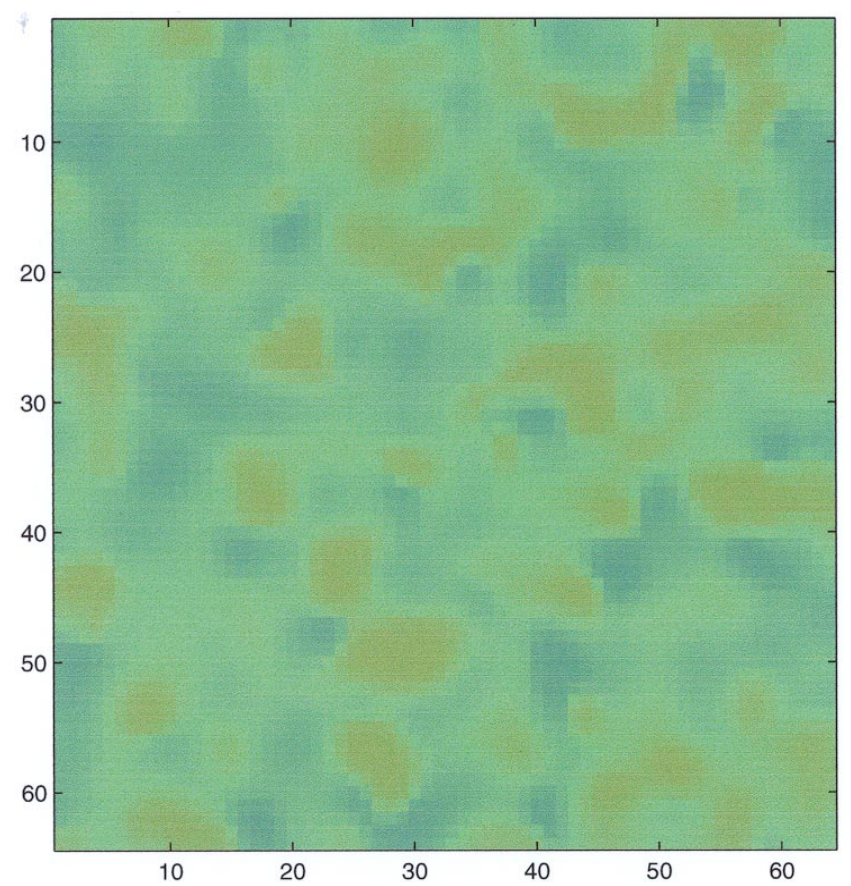

(d)

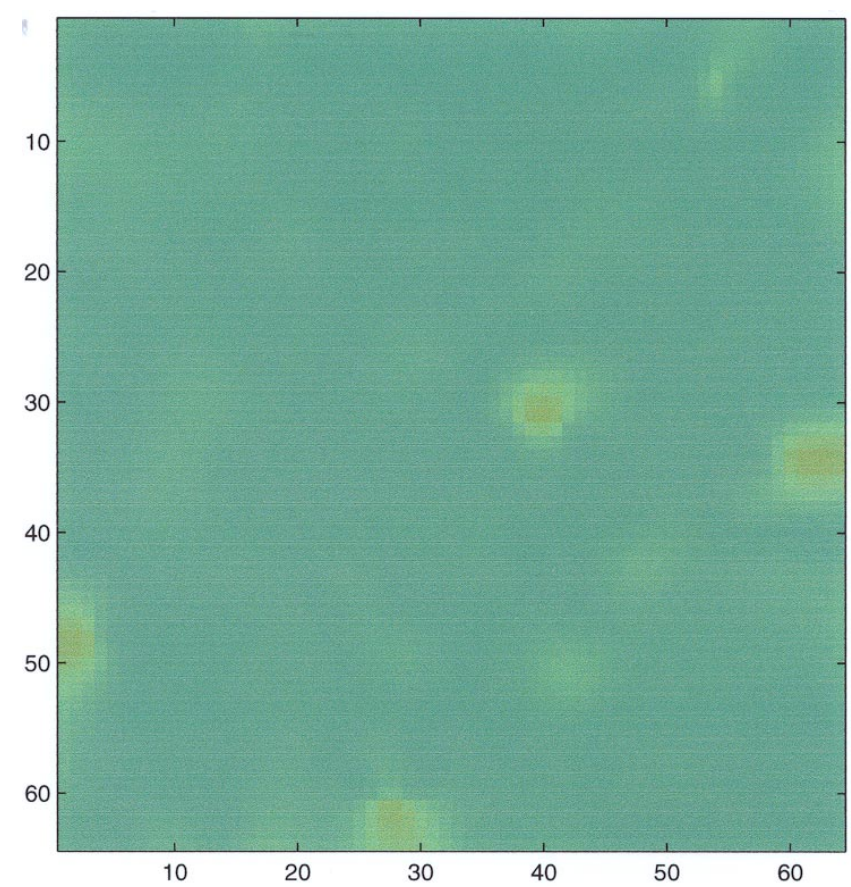

(f)

Fig. 6. (Continued) 


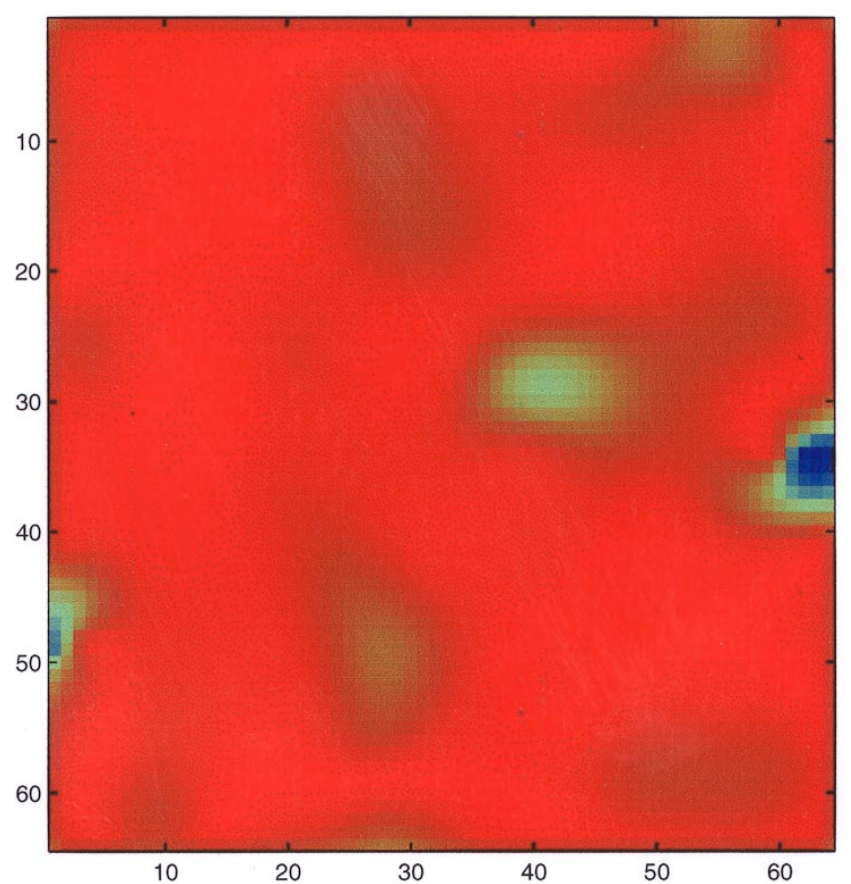

(g)

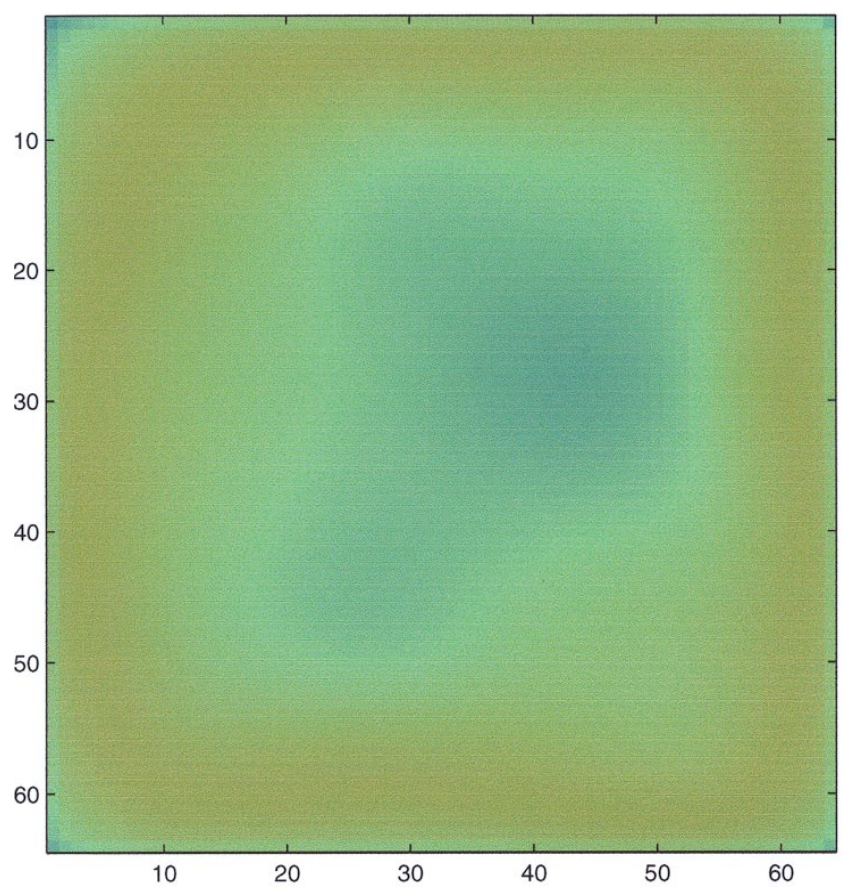

(i)

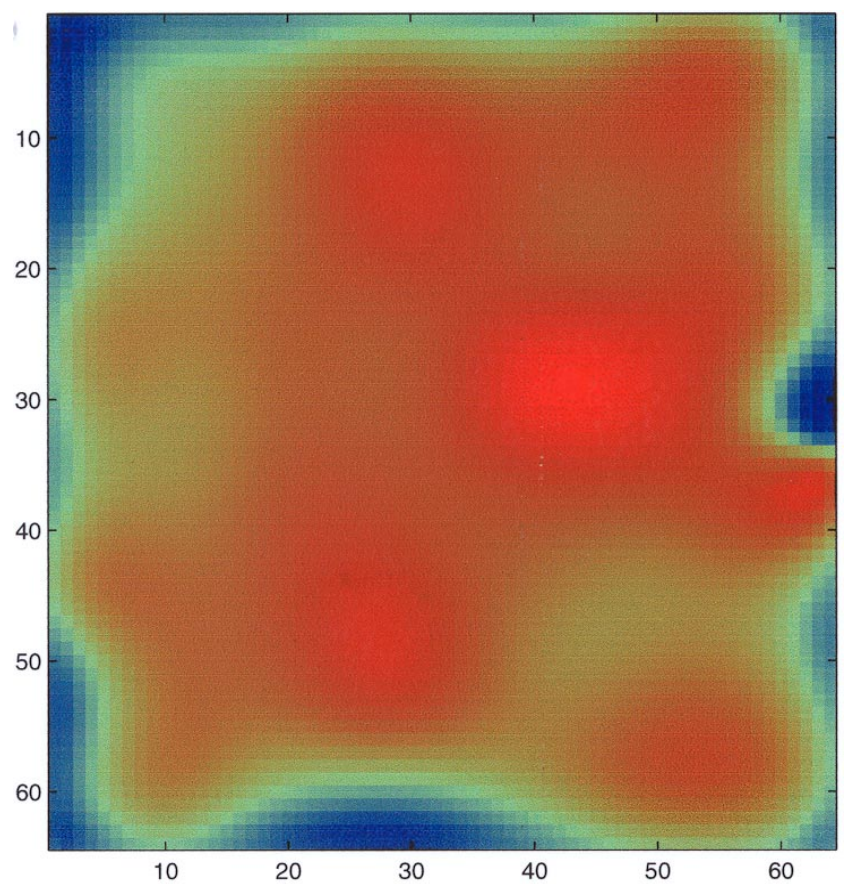

(h)

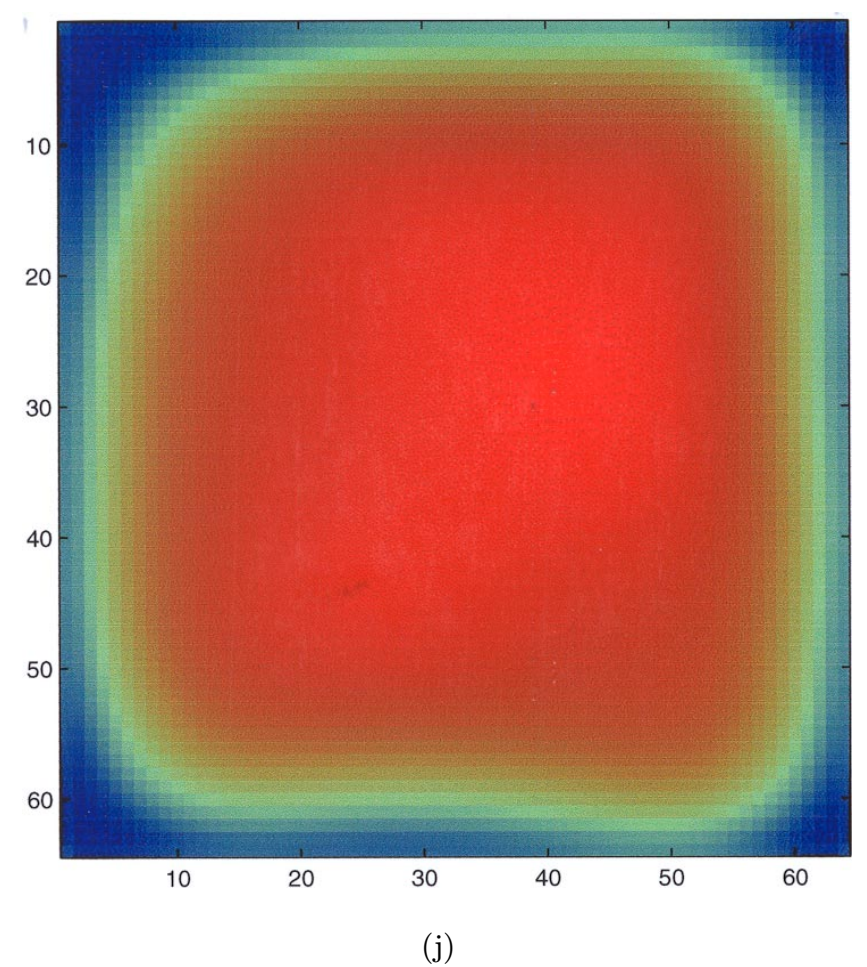

(j)

Fig. 6. (Continued) 


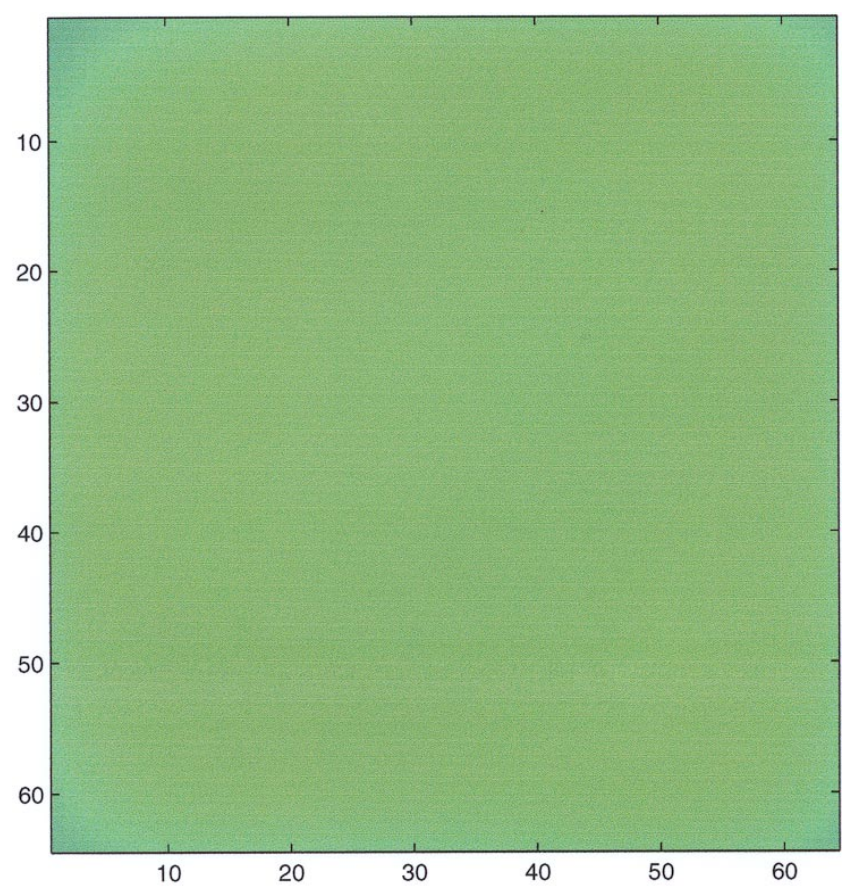

(k)

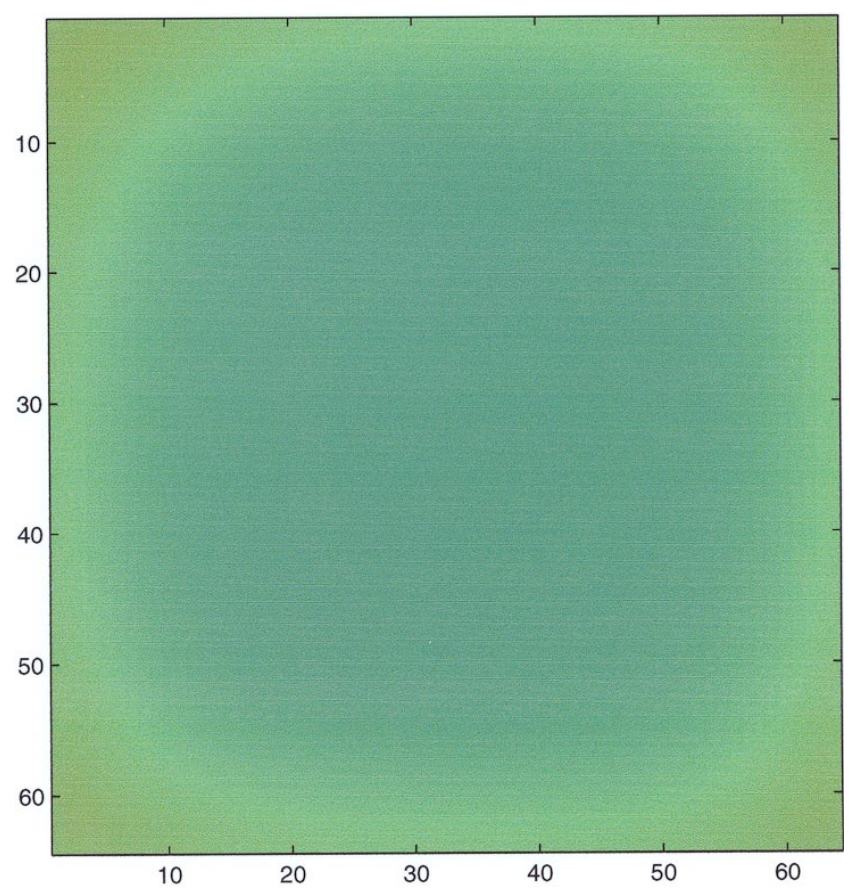

(m)

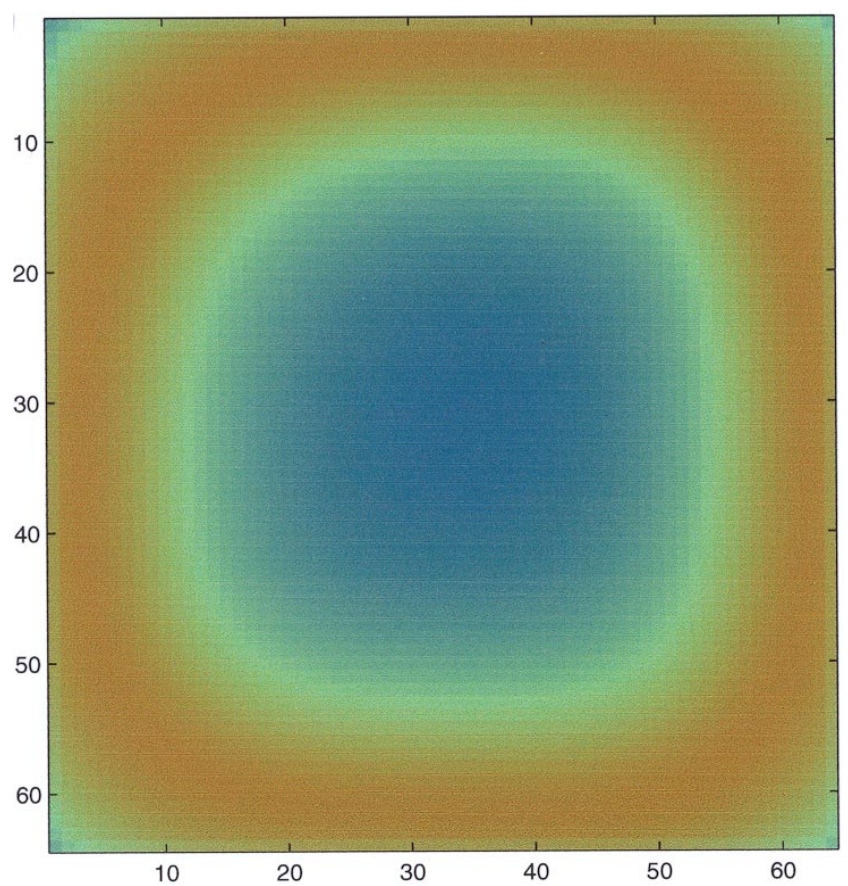

(l)

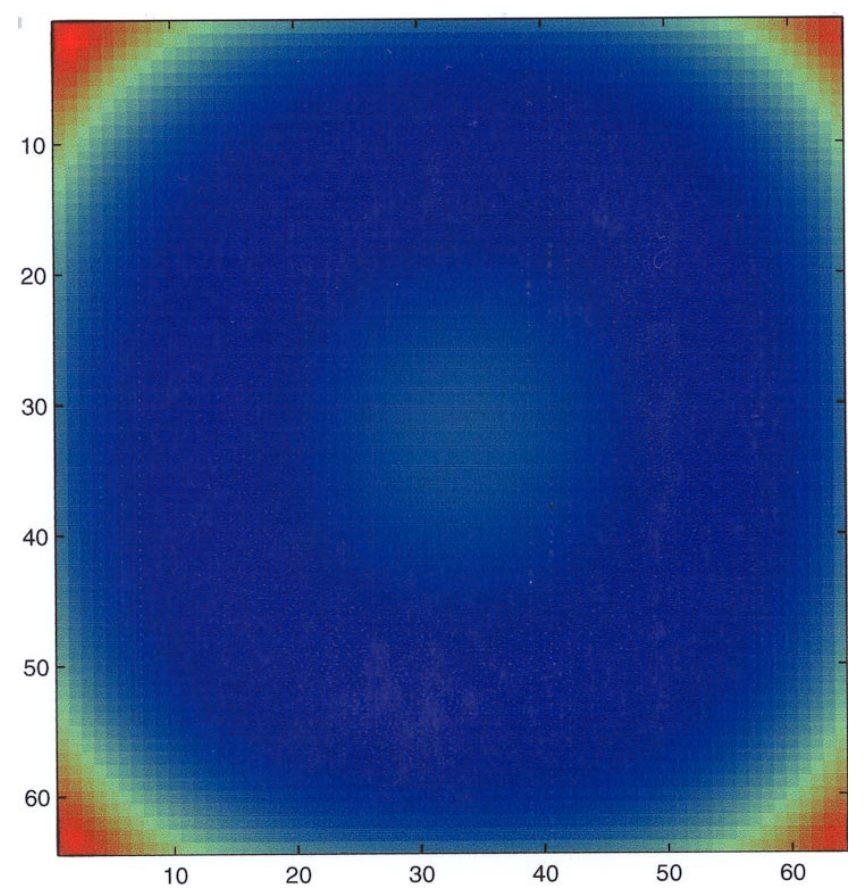

(n)

Fig. 6. (Continued) 


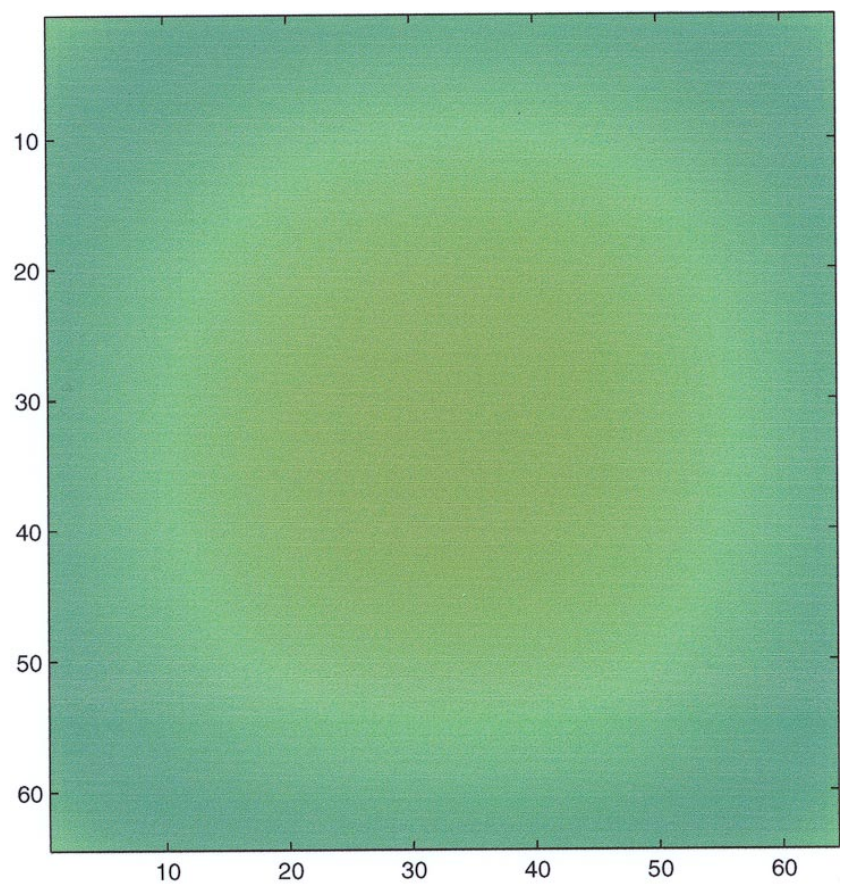

(o)

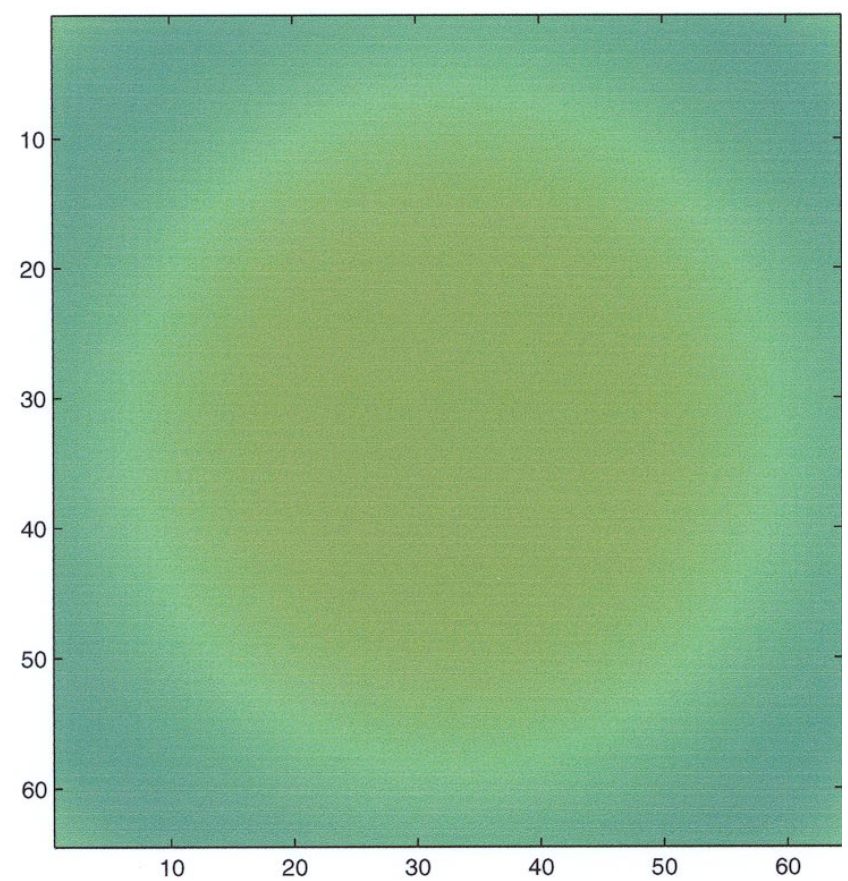

(q)

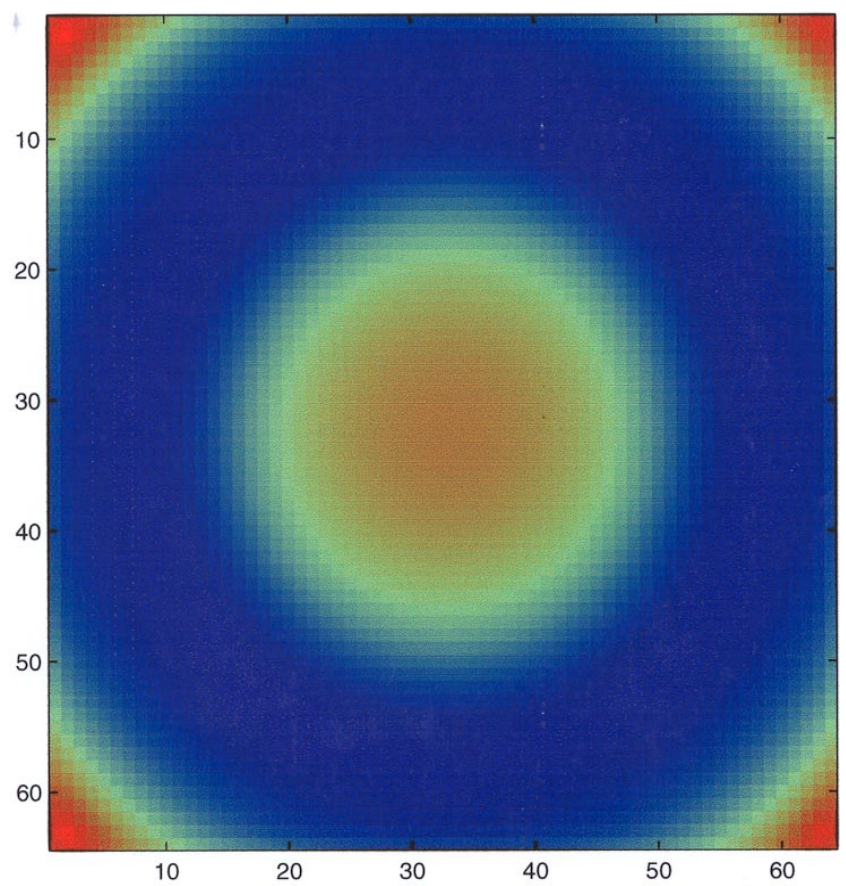

(p)

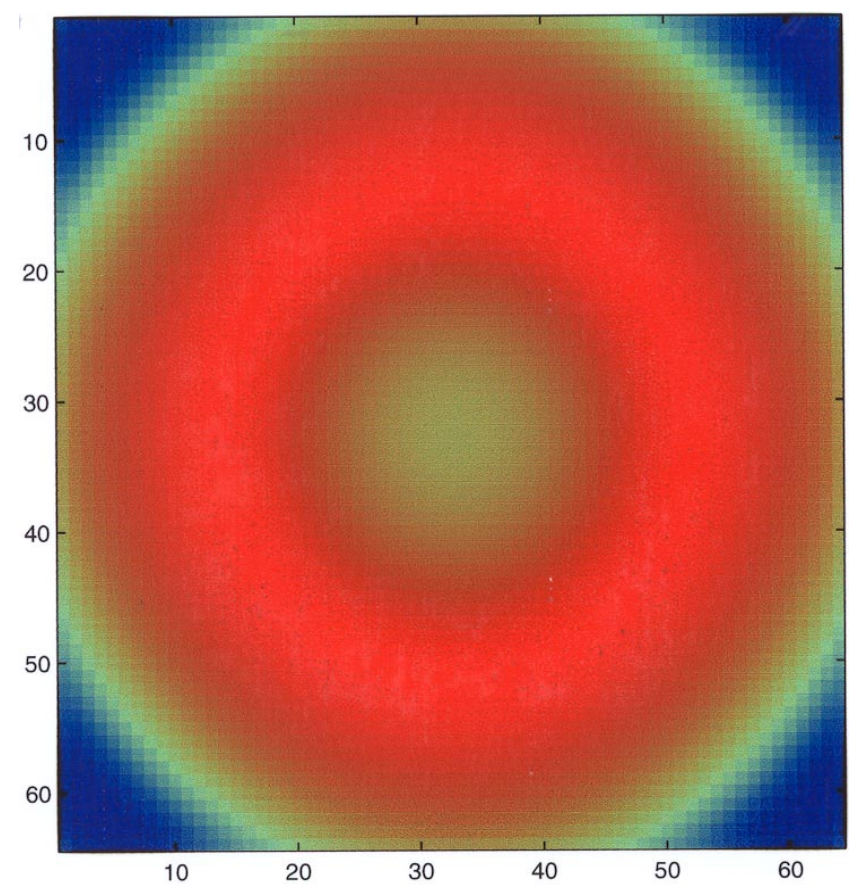

$(\mathrm{r})$

Fig. 6. (Continued) 
1524 T. Yang \& L. O. Chua

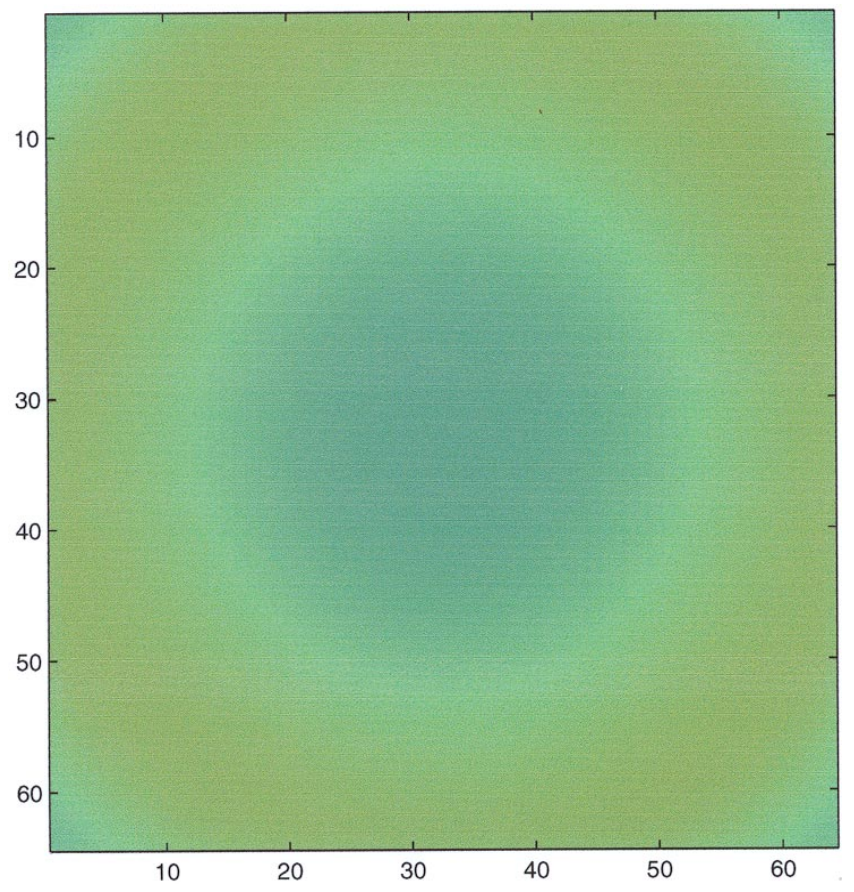

(s)

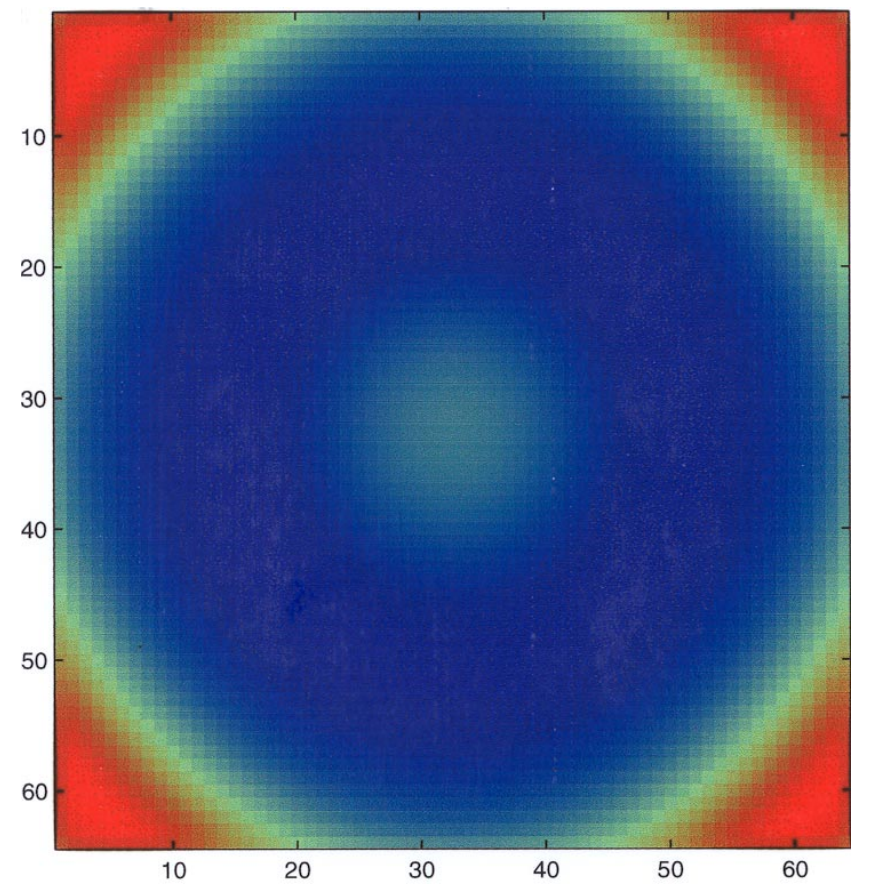

(t)

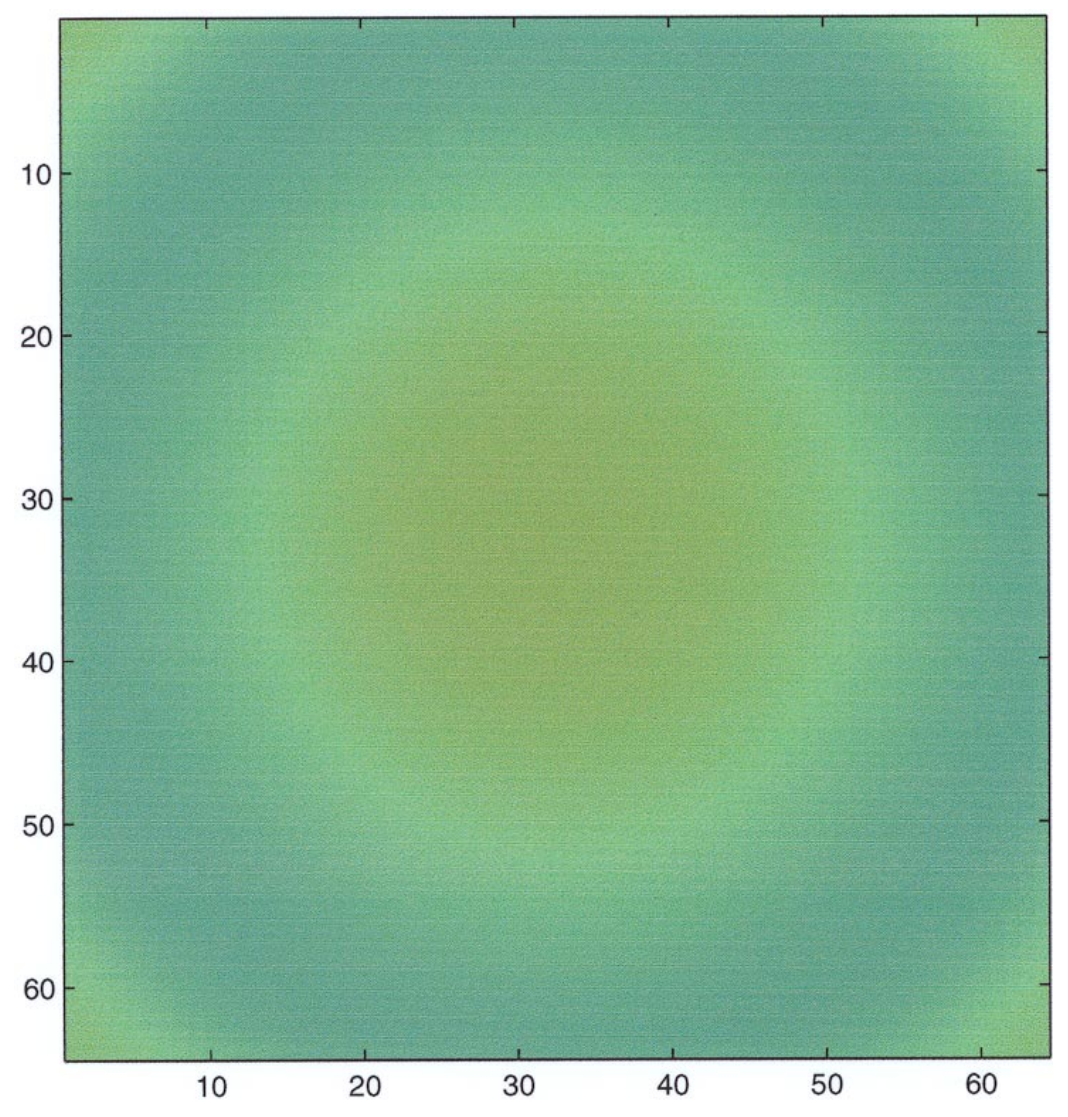

(u)

Fig. 6. (Continued) 
relatively smooth to keep the cell biases for the entire CNN at small values. Observe from the last few figures (e.g. Figs. 6(n)-6(u)) in Fig. 6 that this CNN converges to a kind of "round" target wave. However, this phenomenon is actually caused by the small array size $(64 \times 64)$ of the $\mathrm{CNN}$ being simulated. If we enlarge the array size of the CNN to $200 \times 200$, the behavior will be changed to that shown in Fig. 7. Observe from Figs. 7(g) and 7(h) that the target wave is now square with rounded corners. In this case, we still find that this CNN favors relatively smooth patterns.

The simulation results with parameters $\alpha=$ $-1, b=-10$ and $D_{1}=1$ are shown in Fig. 8 . As shown in Figs. 3(d)-3(f), this CNN is operating in the edge-of-chaos parameter region. The initial conditions for $V_{1}(i, j)$ and $V_{2}(i, j)$ are the same as those shown in Figs. 4(a) and 4(b). Figures 8(a) and $8(\mathrm{~b})$ show $V_{1}(i, j)$ and $V_{2}(i, j)$ at $t=1$, respectively. Figures $8(\mathrm{c})$ and $8(\mathrm{~d})$ show $V_{1}(i, j)$ and $V_{2}(i, j)$ at $t=4$, respectively. Figures $8(\mathrm{e})$ and $8(\mathrm{f})$ show $V_{1}(i, j)$ and $V_{2}(i, j)$ at $t=10$, respectively. Figures $8(\mathrm{~g})$ and $8(\mathrm{~h})$ show $V_{1}(i, j)$ and $V_{2}(i, j)$ at $t=20$, respectively. Figures $8(\mathrm{i})$ and $8(\mathrm{j})$ show $V_{1}(i, j)$ and $V_{2}(i, j)$ at $t=40$, respectively. Comparing with the results shown in Fig. 6 we can see that this CNN converges to a different kind of patterns with big variations from even nearby regions. This is because the edge-of-chaos parameter region shown in Figs. 3(d)-3(f) is separated into two regions when $\alpha=-1$ with the cell biases $I^{*}$ located in the vicinity of \pm 1.3 . Observe that when $I^{*}$ is too small the CNN will be unstable, and the smooth regions in the output of this CNN cannot survive over a long period of time because the instability of the cells will push the states into the edge-of-chaos, or the restricted locally passive regions. On the other hand, patterns with many high-frequency energy components also cannot survive for very long because the over-damping effects of the restricted local passivity will dissipate the high-frequency energy rapidly and squeeze the cell states back into the edge-of-chaos regions. Only medium-sized patterns can survive over a long period of time, as depicted in Fig. 9 for a $200 \times 200$ array size.

The simulation results with parameters $\alpha=$ $-18, b=-10$ and $D_{1}=1$ are shown in Fig. 10 . As shown in Figs. 3(d)-3(f), this CNN is operating in the edge-of-chaos parameter region. The initial conditions for $v_{1}(i, j)$ and $v_{2}(i, j)$ are the same as those shown in Figs. 4(a) and 4(b). Observe that with a $64 \times 64$ array size, this CNN converges to a target wave.

In Fig. 11 we show this CNN with a $200 \times 200$ array size. Observe that in this case the $\mathrm{CNN}$ output is also dominated by a target wave. However, since the CNN size in this case is much bigger than the spatial wavelength, ${ }^{3}$ we do not see the surprisingly ideal circular shape as depicted in Fig. 10 where the CNN size is only two or three times bigger than the spatial wavelength.

\section{Two-Port and Second-Order CNN Cells: Examples}

In this section, we study CNNs made of two-port and second-order cells. Let the state equations of each cell be given by

$$
\begin{aligned}
& \dot{V}_{1}=-V_{1}^{3}+(\alpha+b) V_{1}-b V_{2}+I_{1}^{*}, \\
& \dot{V}_{2}=V_{1}-V_{2}+I_{2}^{*}
\end{aligned}
$$

where $I_{1}^{*} \in(-\infty, \infty)$ and $I_{2}^{*} \in(-\infty, \infty)$ denote two external forcings (also called "port current biases" in view of their circuit theory interpretations) due to couplings from neighboring cells. The equilibrium point is given by

$$
\begin{aligned}
V_{2}^{*} & =V_{1}^{*}+I_{2}^{*}, \\
V_{1}^{* 3}-\alpha V_{1}^{*}-I^{*} & =0, \\
I^{*} & \triangleq b I_{2}^{*}-I_{1}^{*} .
\end{aligned}
$$

Notice that $V_{1}^{*}$ has the same form as that of oneport first-order cells. The Jacobian matrix at an equilibrium point $Q$ is given by

$$
J_{Q}=\left(\begin{array}{cc}
-3 V_{1}^{* 2}+\alpha+b & -b \\
1 & -1
\end{array}\right)
$$

with

$$
T=-3 V_{1}^{* 2}+\alpha+b-1, \Delta=3 V_{1}^{* 2}-\alpha .
$$

1. From P1 we have

$$
-3 V_{1}^{* 2}+\alpha+b-1 \leq 0 \quad \mathbb{A N D} \quad 3 V_{1}^{* 2}-\alpha \geq 0
$$

\footnotetext{
${ }^{3}$ Here the "spatial wavelength" gives only a rough intuitive description of the visible features of the target wave, such as one-half of the diameter of the blue region in Fig. 10(o).
} 


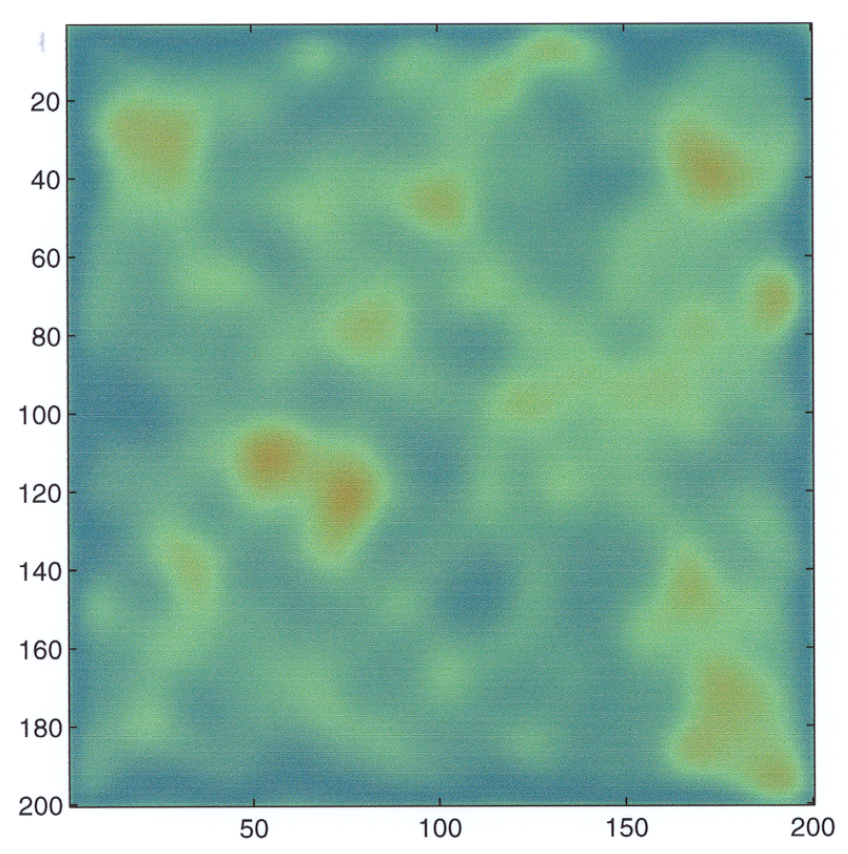

(a)

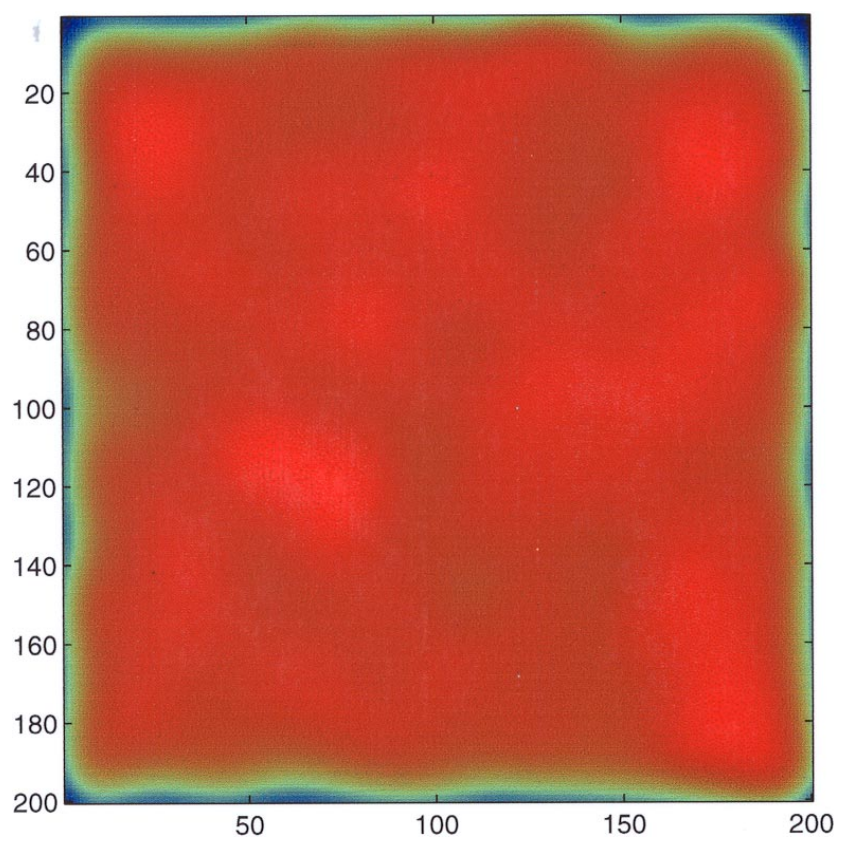

(c)

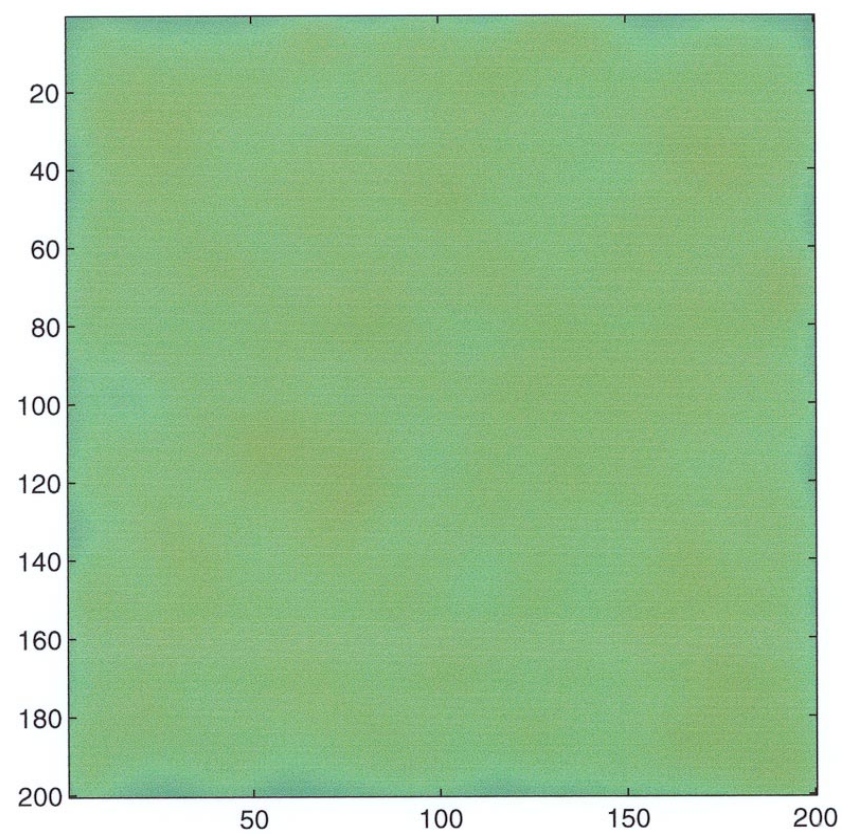

(b)

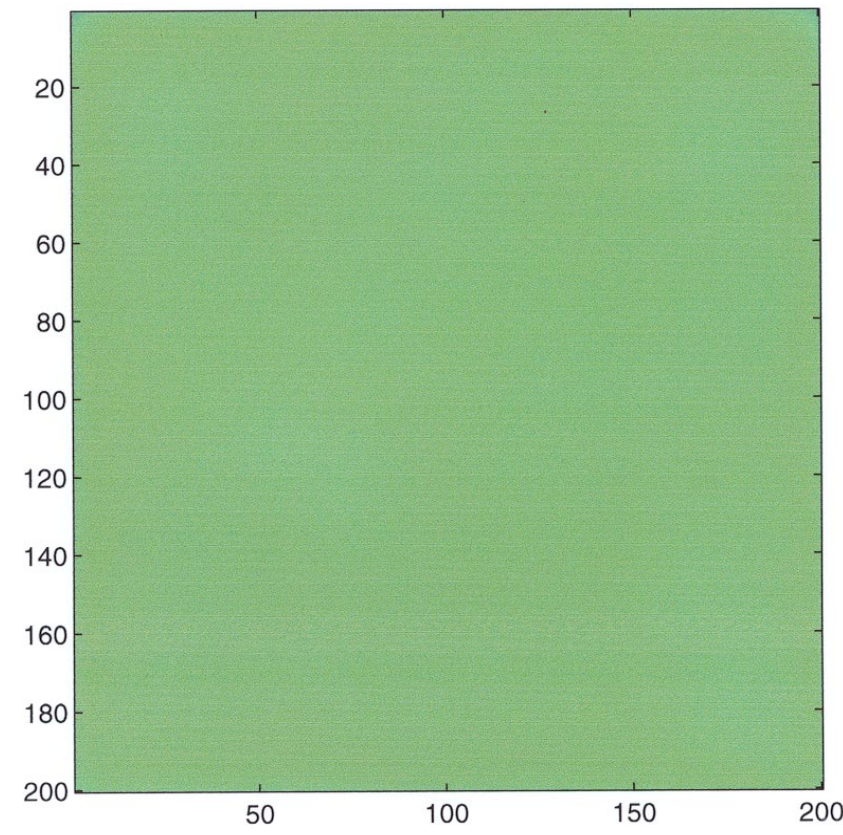

(d)

Fig. 7. Simulation results of CNN made of one-port second-order cells. The parameters are given by $\alpha=-1, b=-1$ and $D_{1}=1$. The cells are operating in the edge-of-chaos regions. (a) $V_{1}(i, j)$ at $t=50$. (b) $V_{2}(i, j)$ at $t=50$. (c) $V_{1}(i, j)$ at $t=100$. (d) $V_{2}(i, j)$ at $t=100$. (e) $V_{1}(i, j)$ at $t=500$. (f) $V_{2}(i, j)$ at $t=500$. (g) $V_{1}(i, j)$ at $t=1000$. (h) $V_{2}(i, j)$ at $t=1000$. 


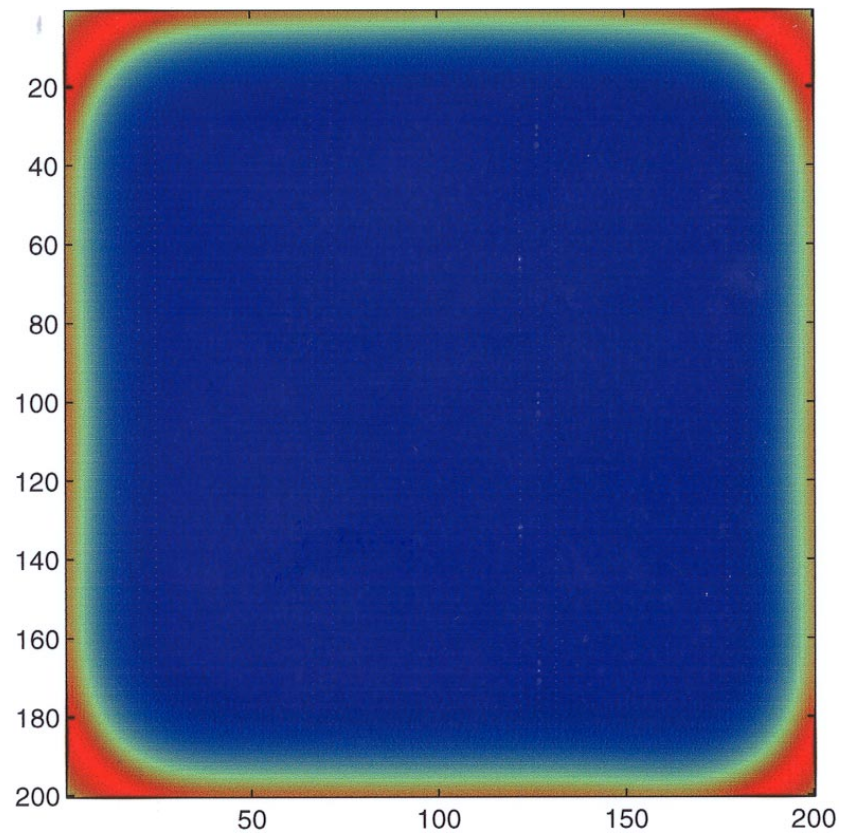

(e)

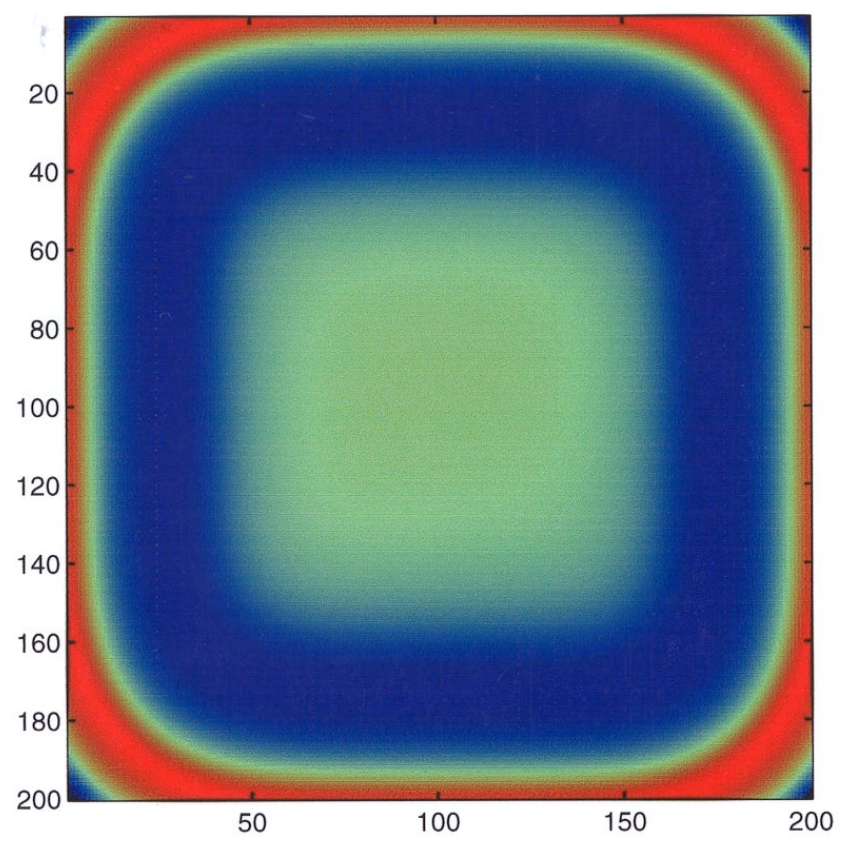

(g)

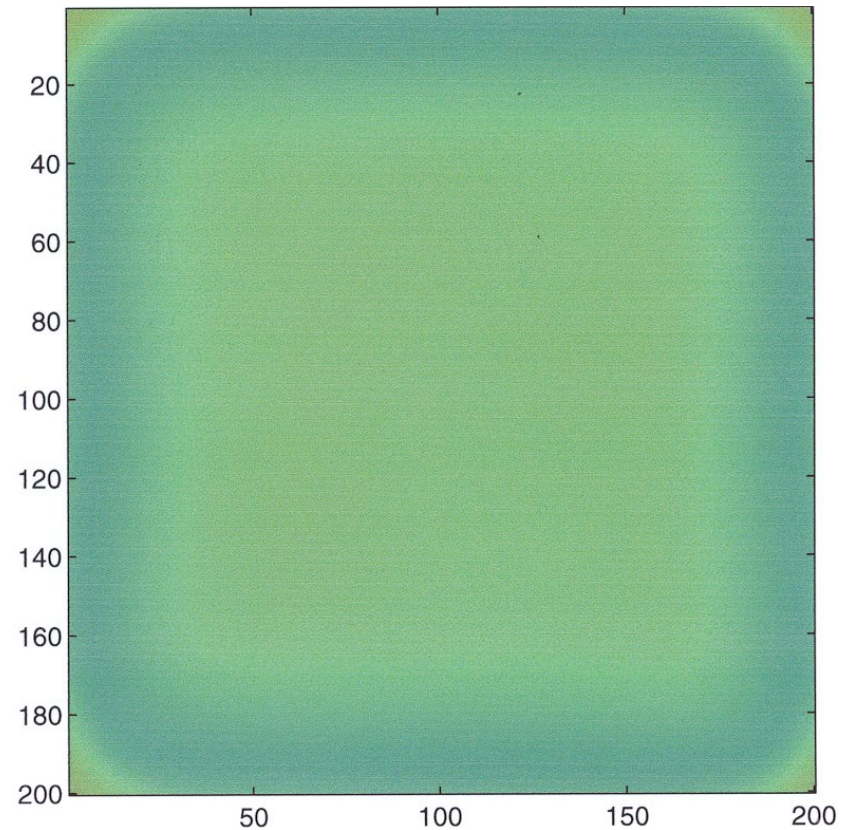

(f)

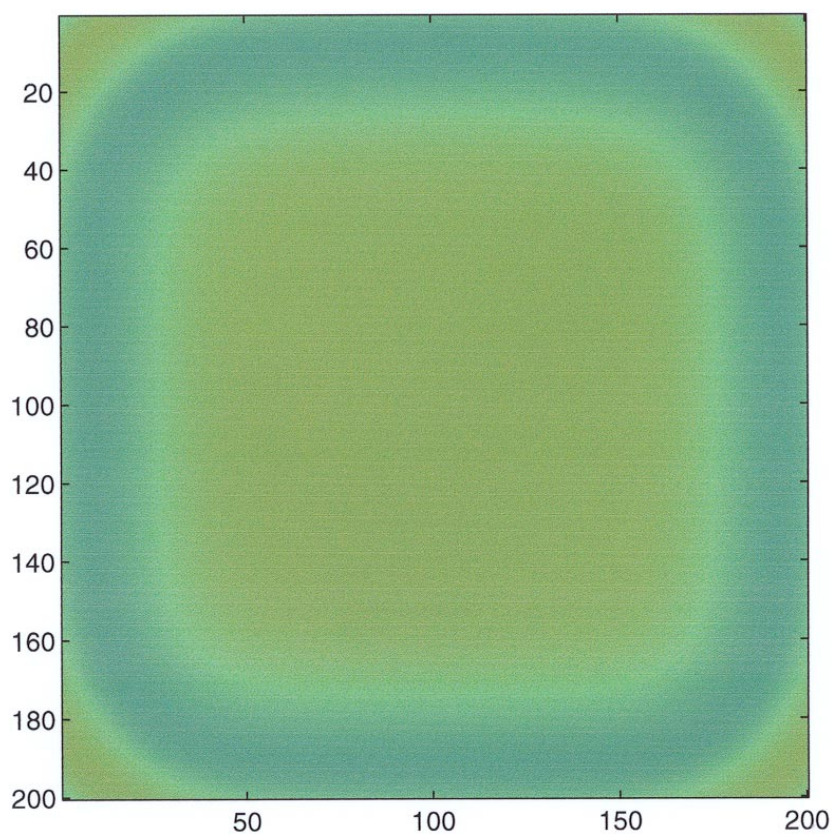

(h)

Fig. 7. (Continued) 


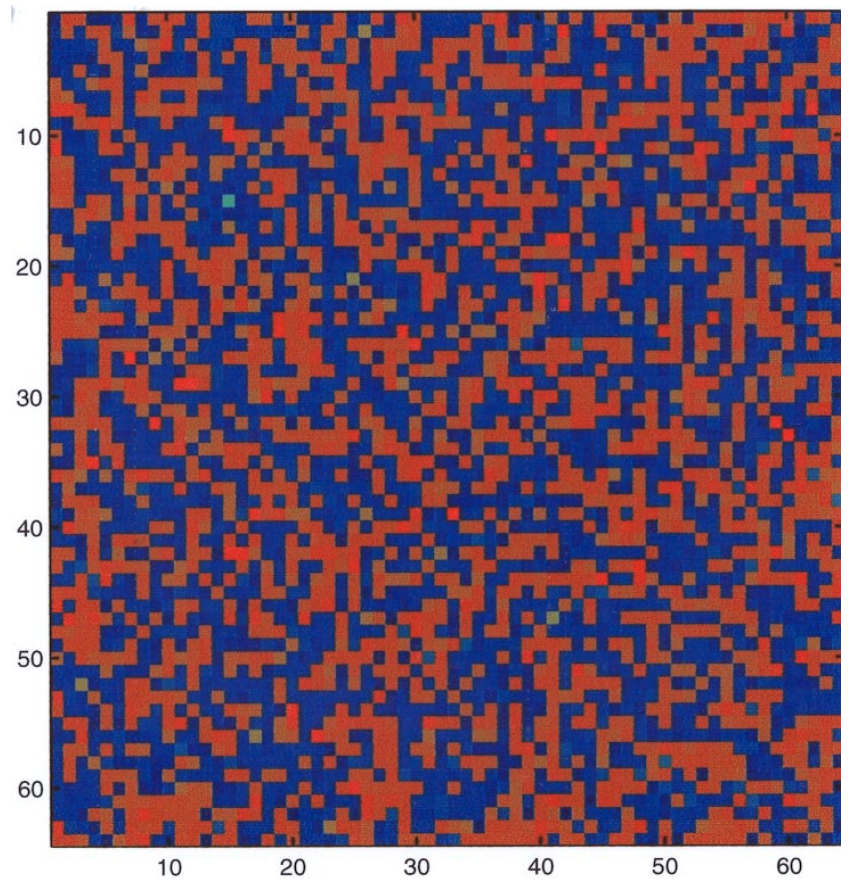

(a)

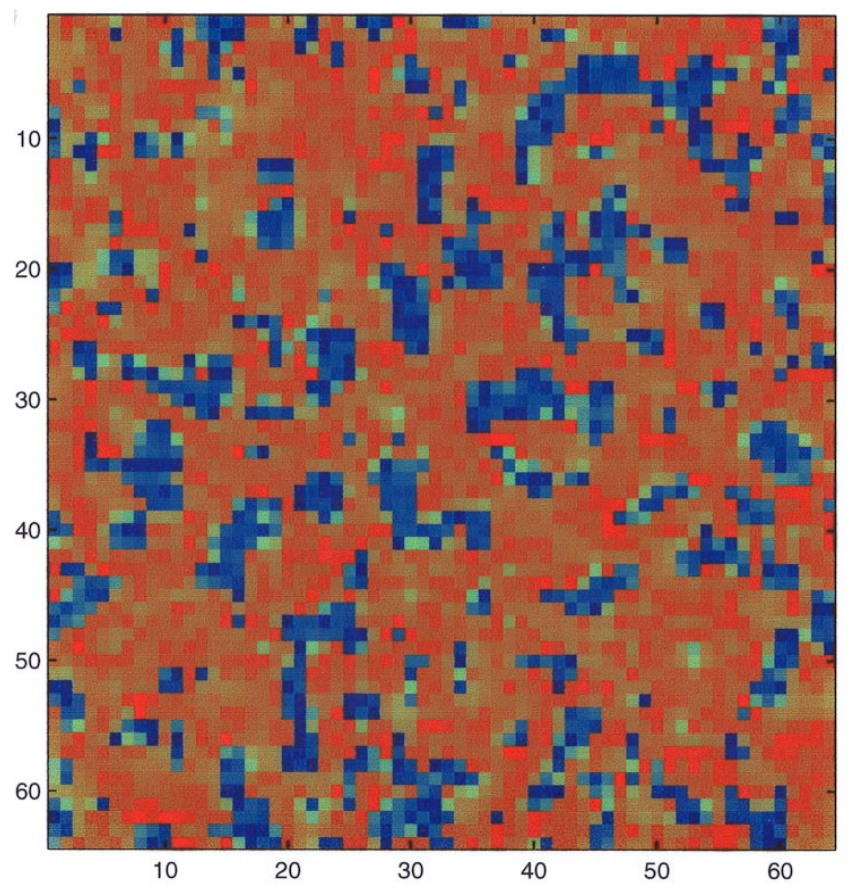

(c)

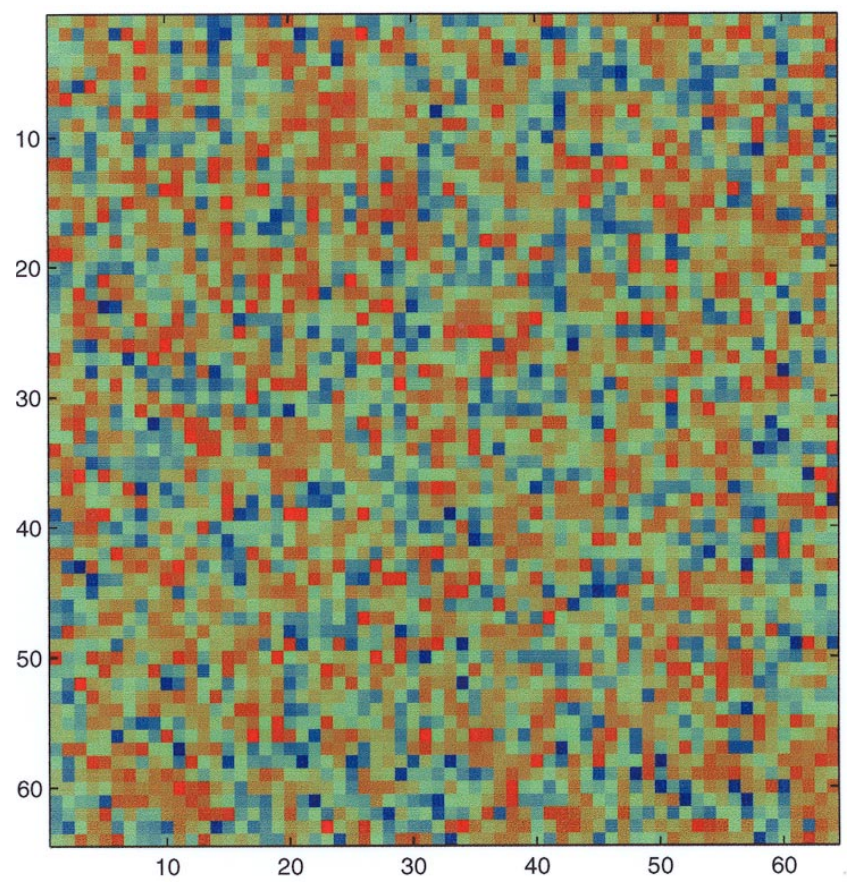

(b)

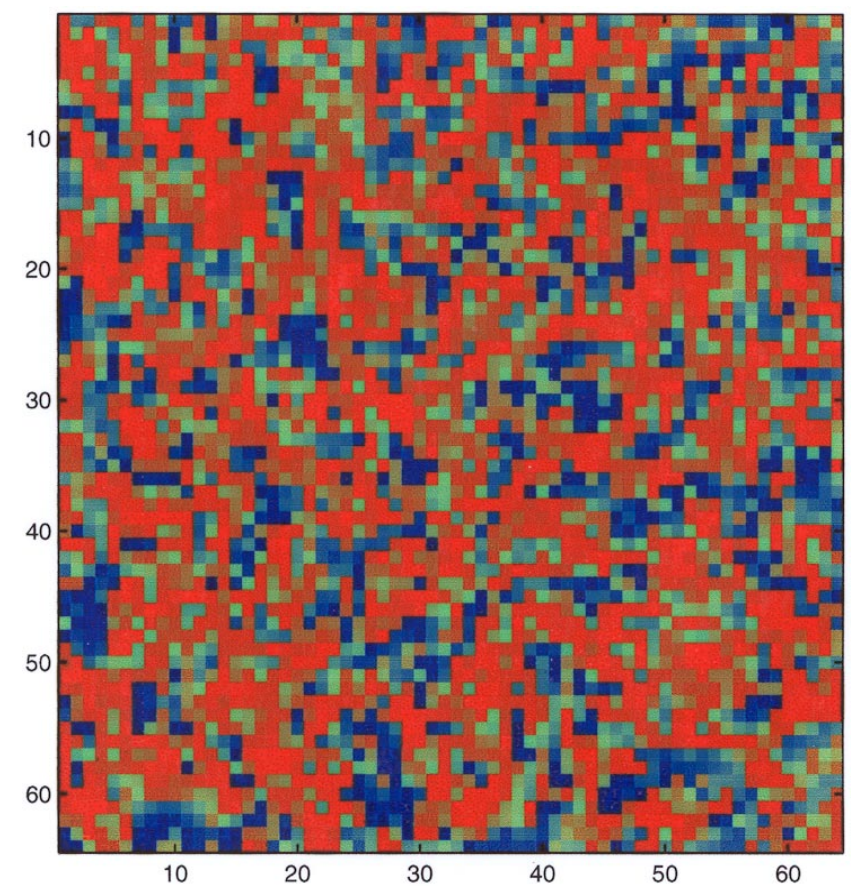

(d)

Fig. 8. Simulation results of CNN made of one-port second-order cells. The parameters are given by $\alpha=-1, b=-10$ and $D_{1}=1$. The cells are operating in the edge-of-chaos regions. (a) $V_{1}(i, j)$ at $t=1$. (b) $V_{2}(i, j)$ at $t=1$. (c) $V_{1}(i, j)$ at $t=4$. (d) $V_{2}(i, j)$ at $t=4$. (e) $V_{1}(i, j)$ at $t=10$. (f) $V_{2}(i, j)$ at $t=10$. (g) $V_{1}(i, j)$ at $t=20$. (h) $V_{2}(i, j)$ at $t=20$. (i) $V_{1}(i, j)$ at $t=40$. (j) $V_{2}(i, j)$ at $t=40$. (k) $V_{1}(i, j)$ at $t=100$. (l) $V_{2}(i, j)$ at $t=100$. (m) $V_{1}(i, j)$ at $t=200$. (n) $V_{2}(i, j)$ at $t=200$. (o) $V_{1}(i, j)$ at $t=300$. (p) $V_{2}(i, j)$ at $t=300$. (q) $V_{1}(i, j)$ at $t=400$. (r) $V_{2}(i, j)$ at $t=400$. (s) $V_{1}(i, j)$ at $t=600$. (t) $V_{2}(i, j)$ at $t=600$. 


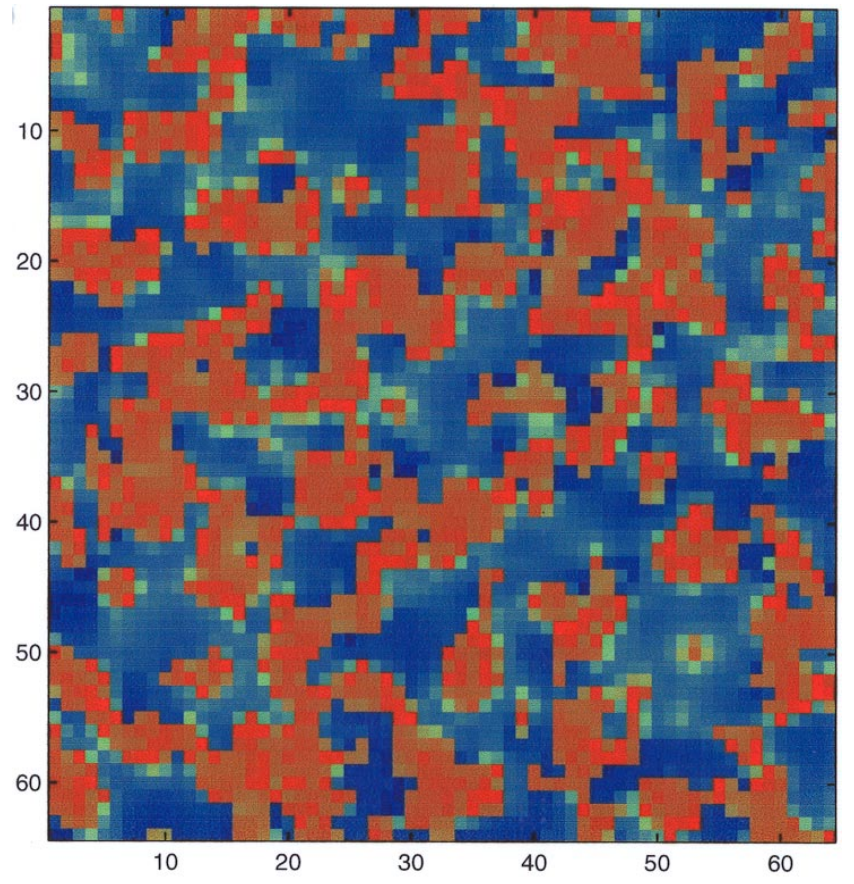

(e)

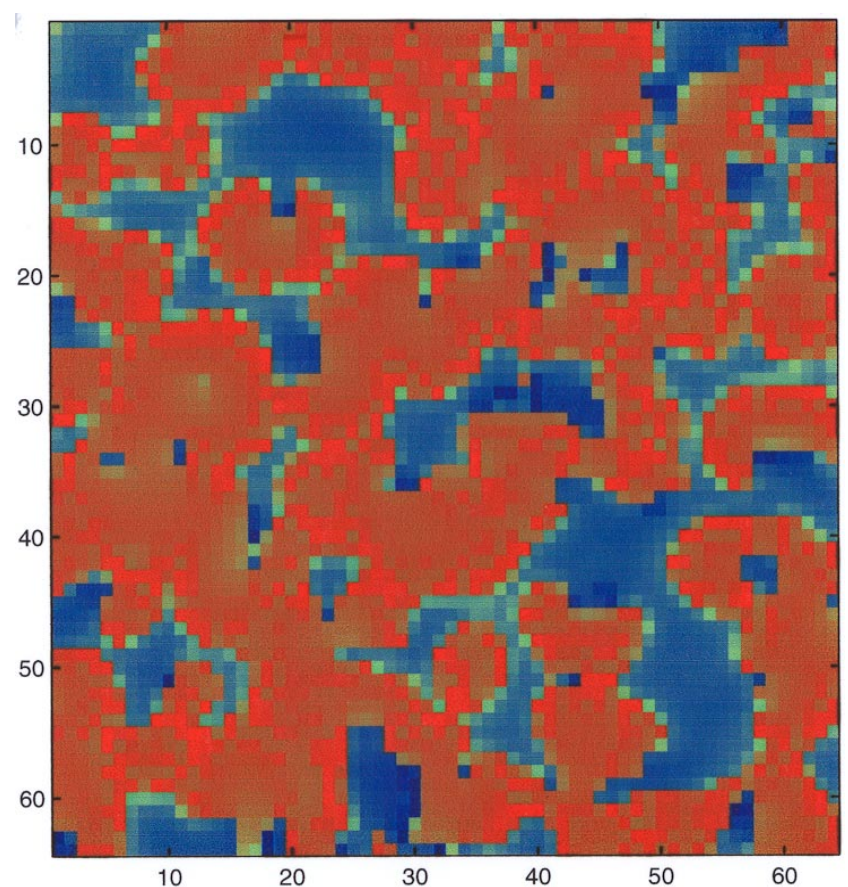

(g)

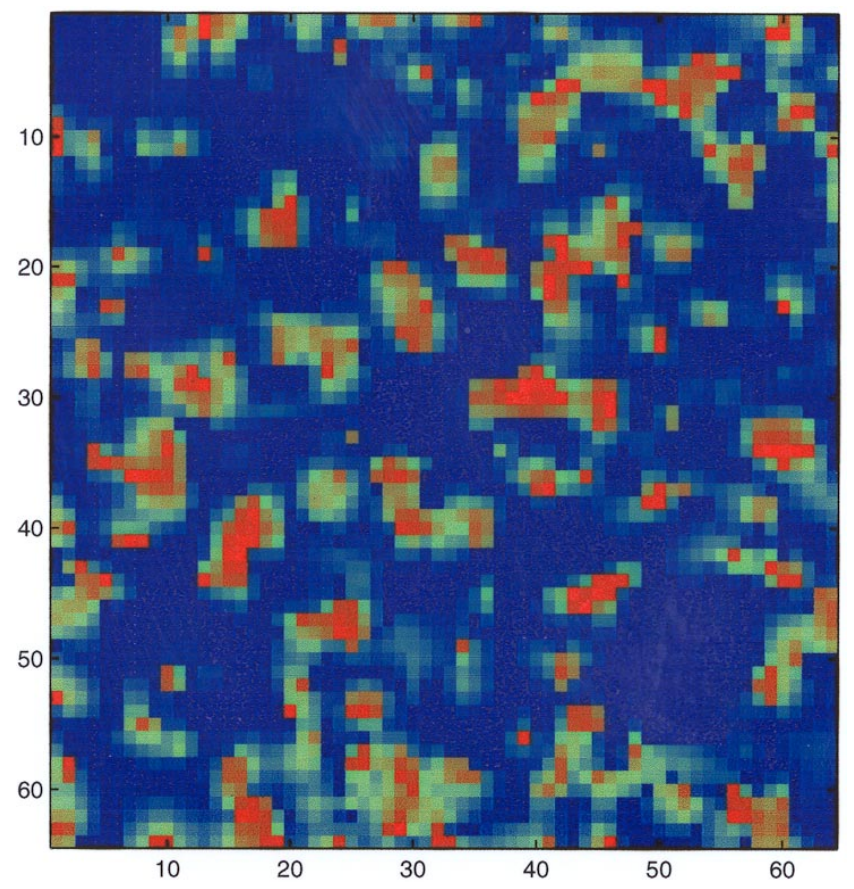

(f)

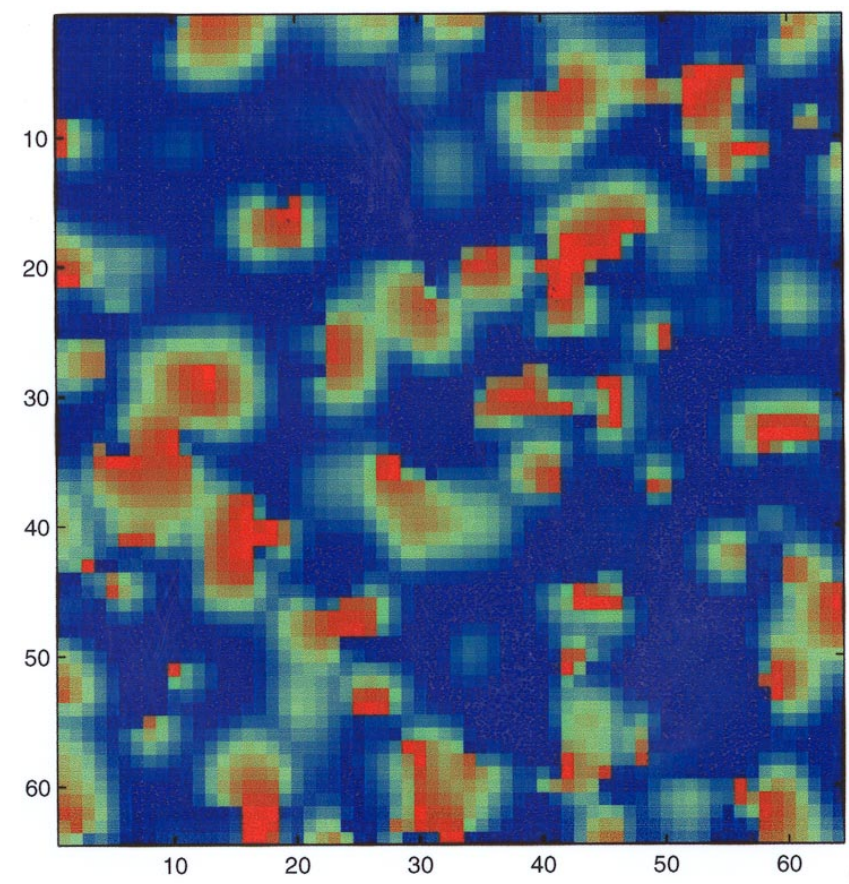

(h)

Fig. 8. (Continued) 


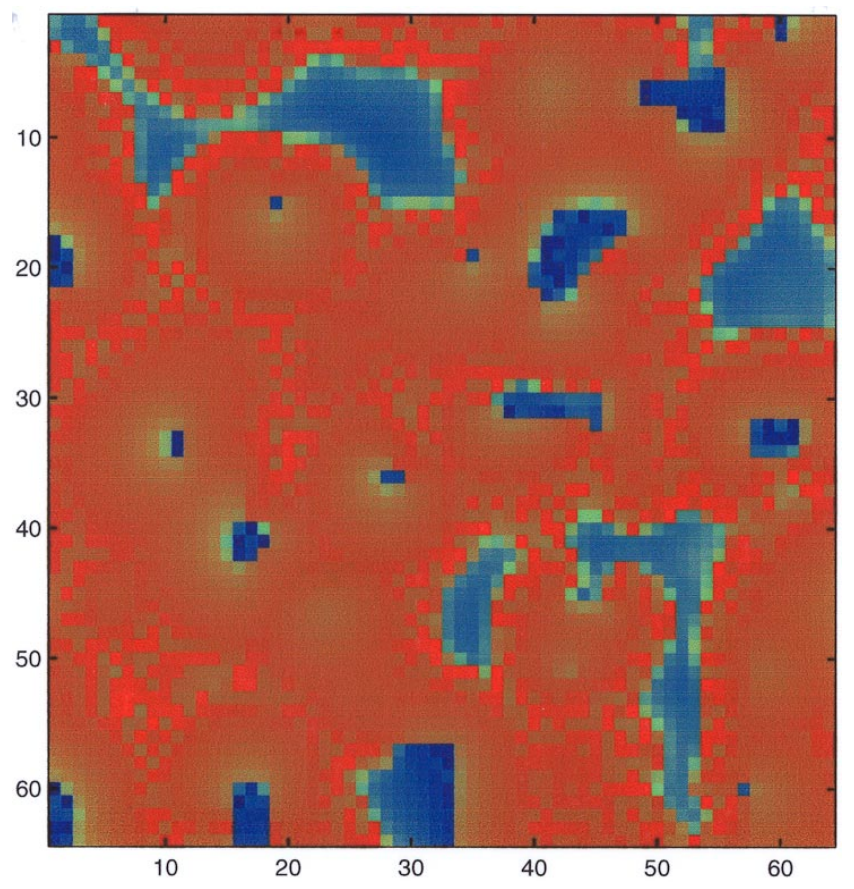

(i)

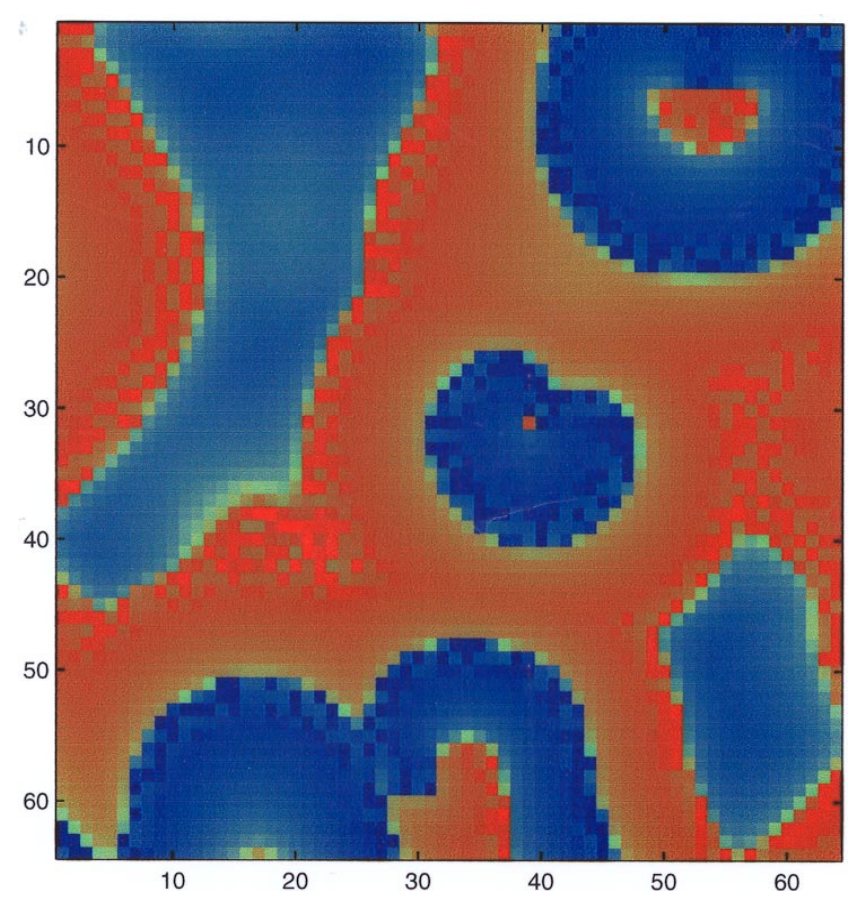

(k)

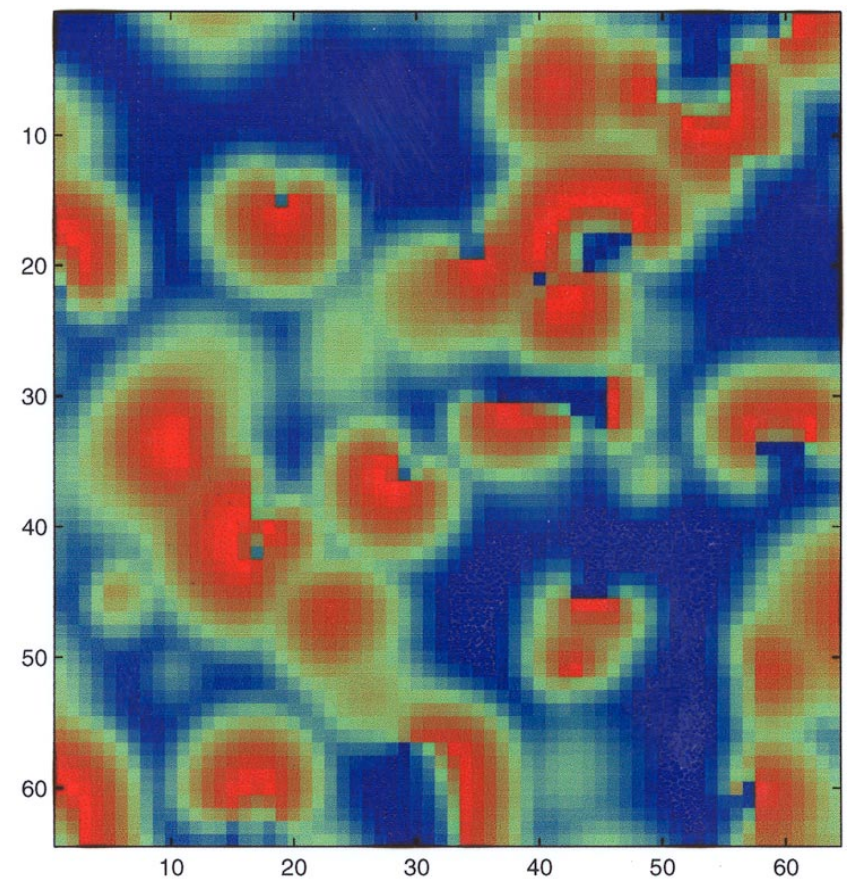

(j)

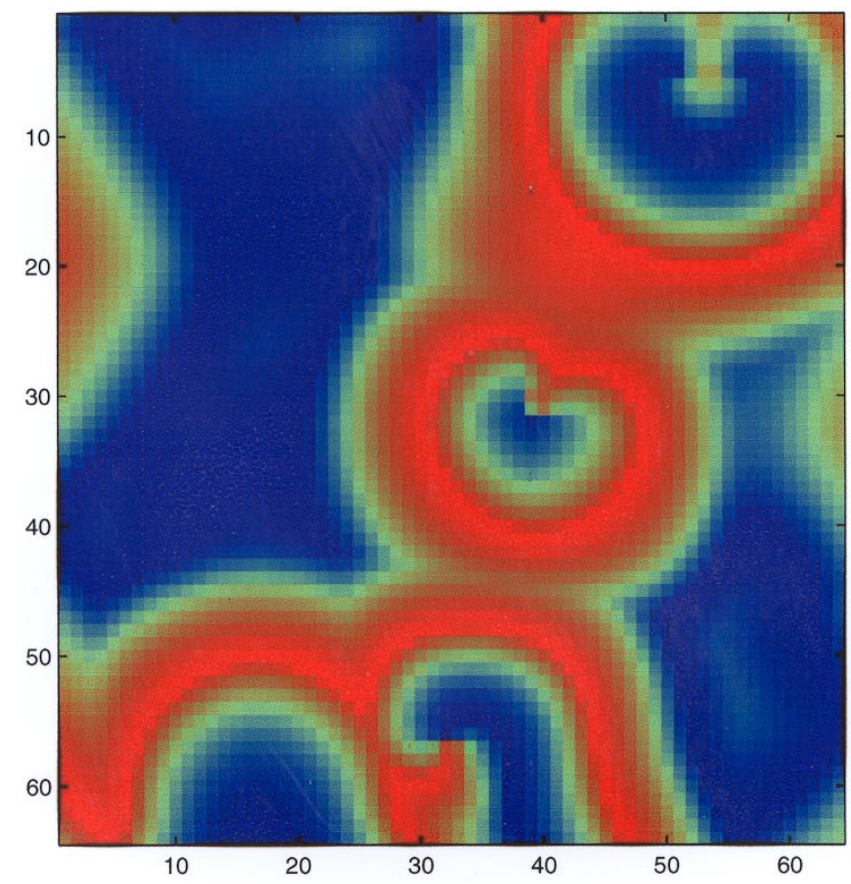

(l)

Fig. 8. (Continued) 


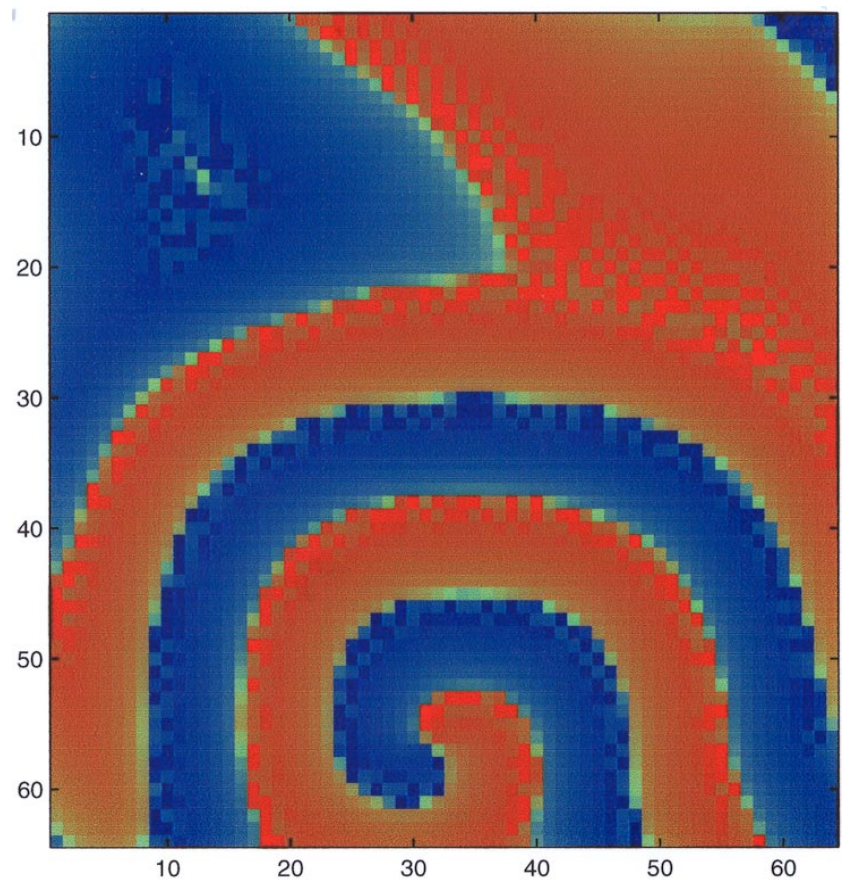

(m)

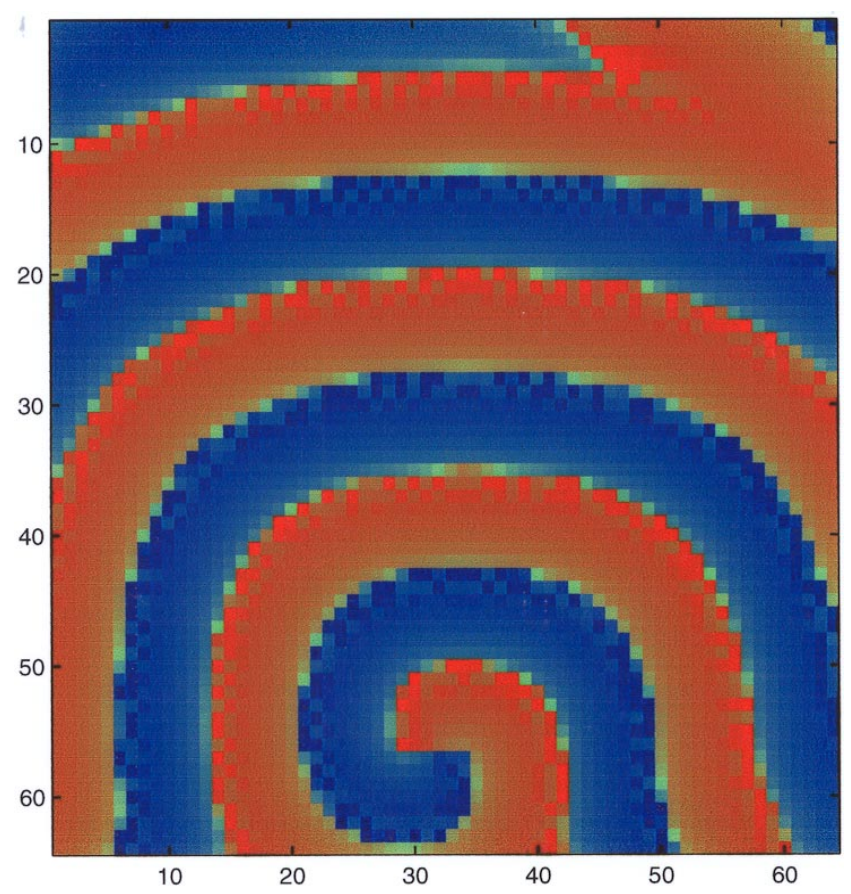

(o)

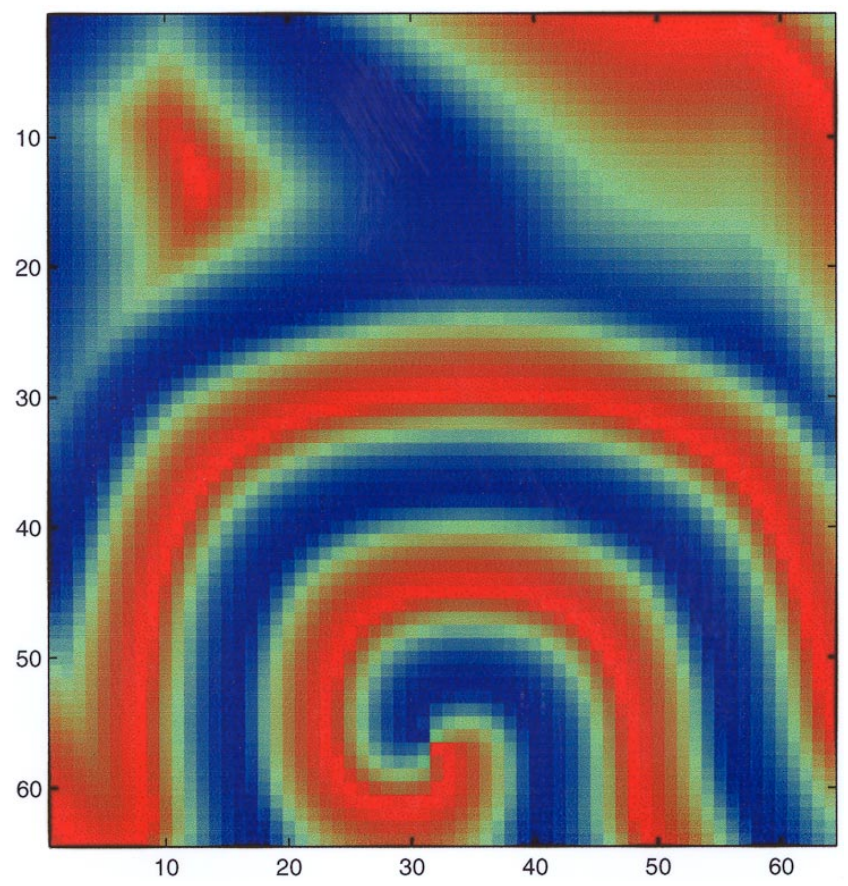

(n)

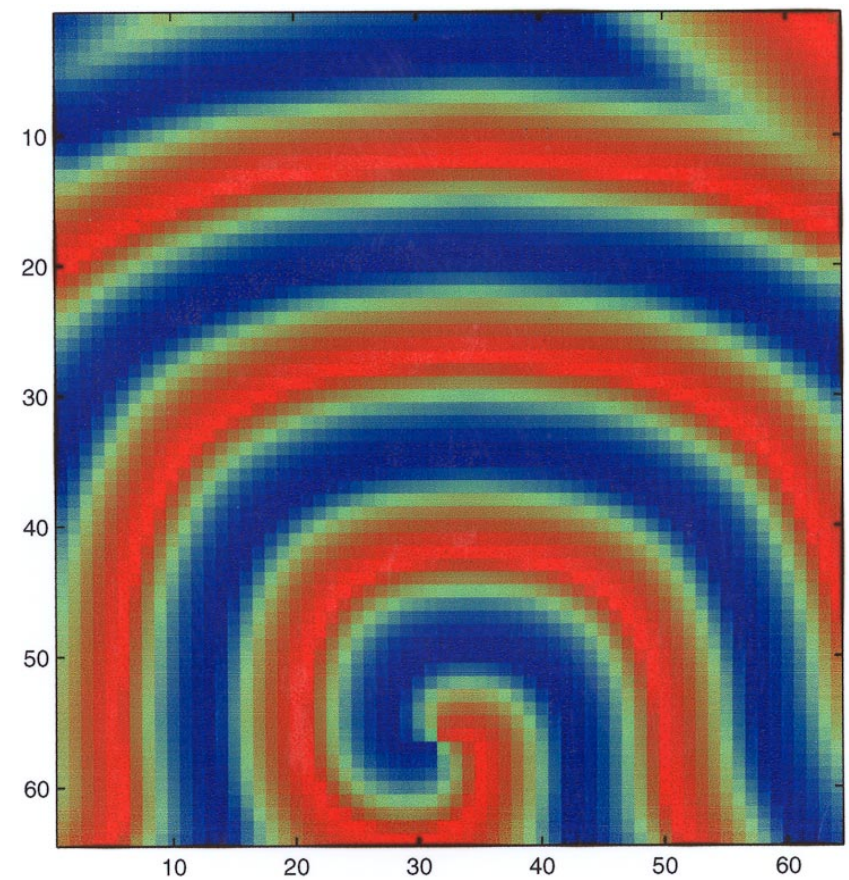

(p)

Fig. 8. (Continued) 


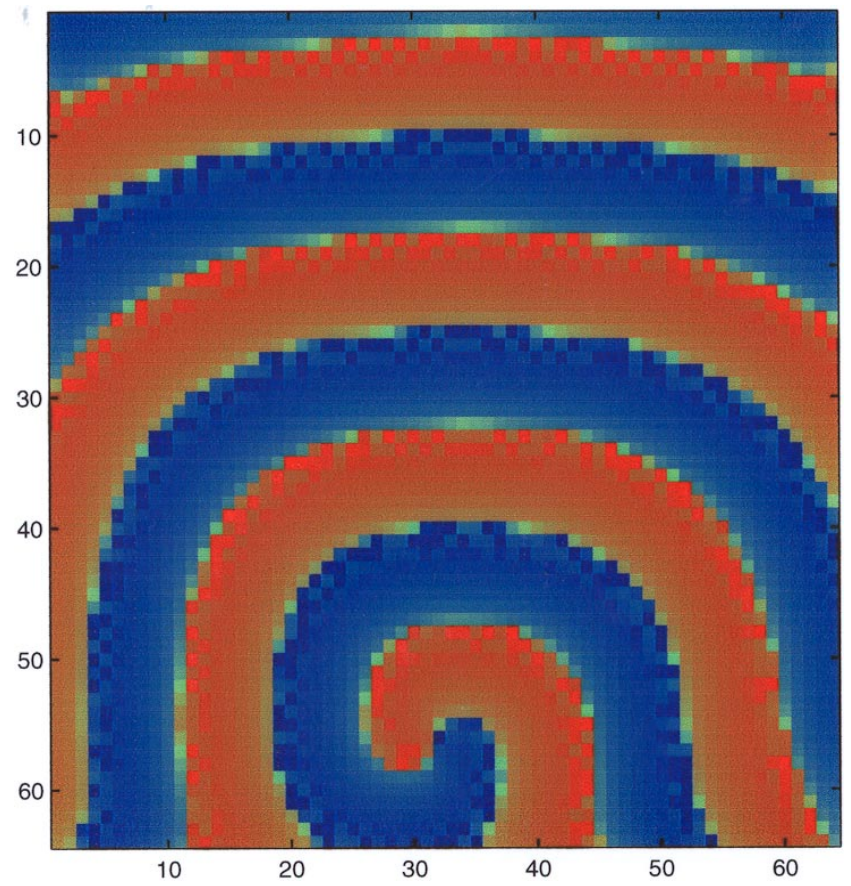

(q)

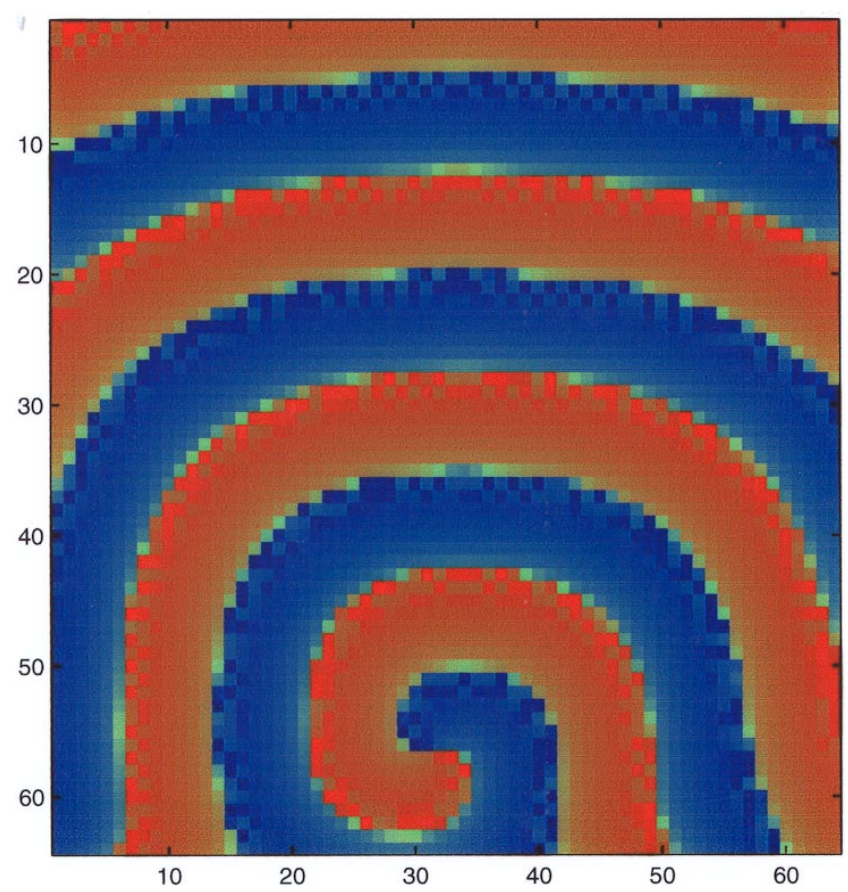

(s)

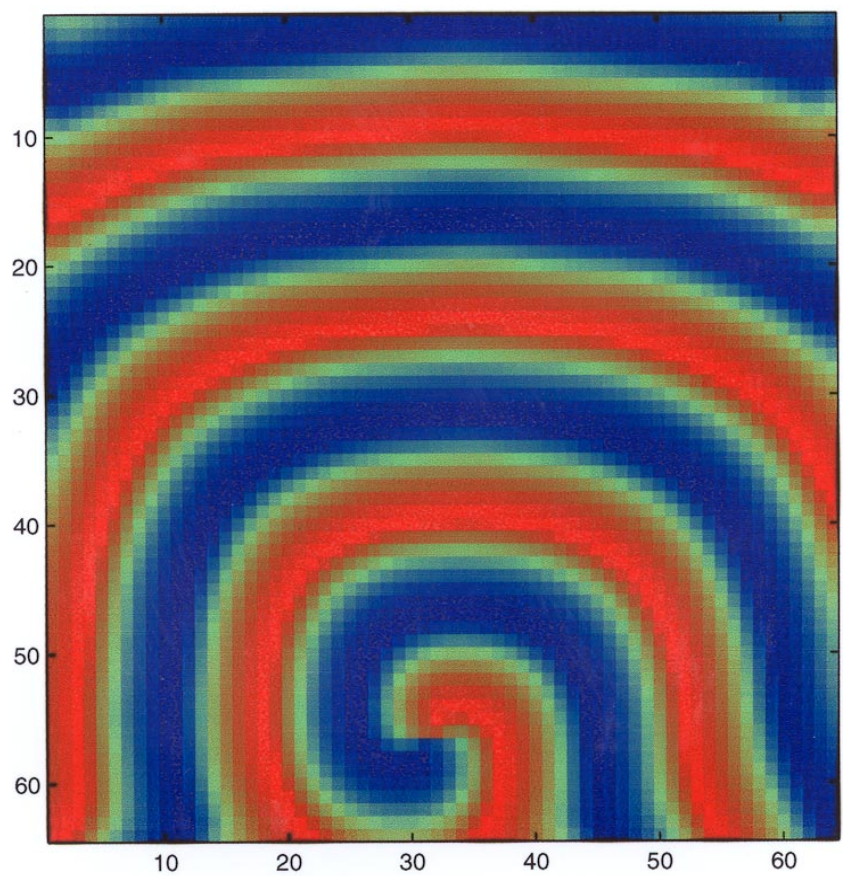

$(\mathrm{r})$

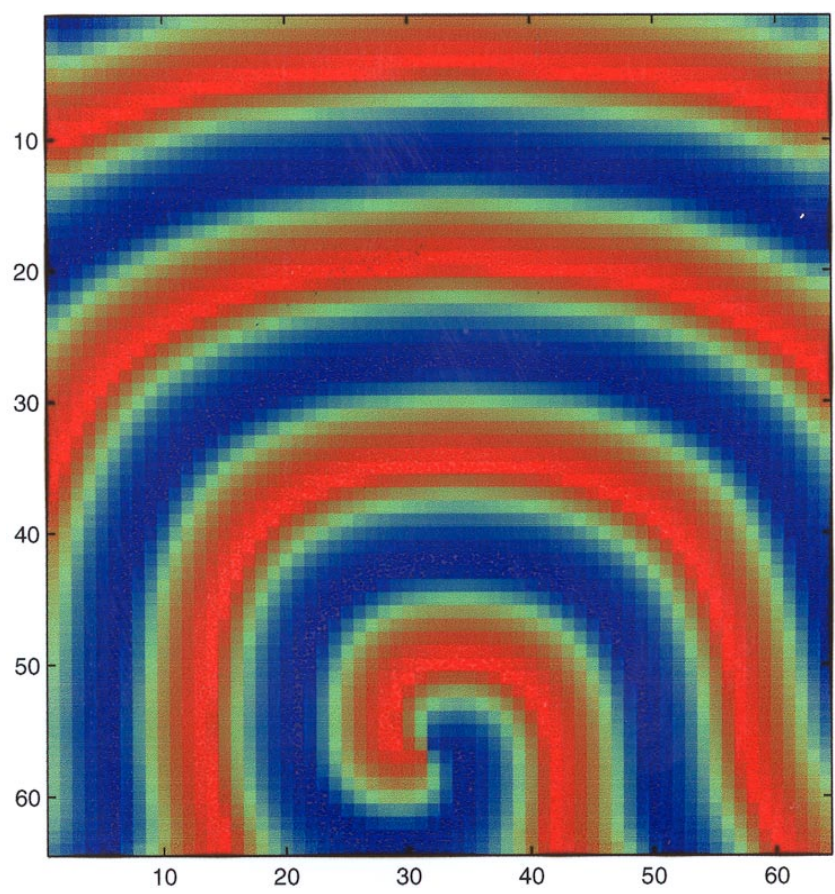

(t)

Fig. 8. (Continued) 


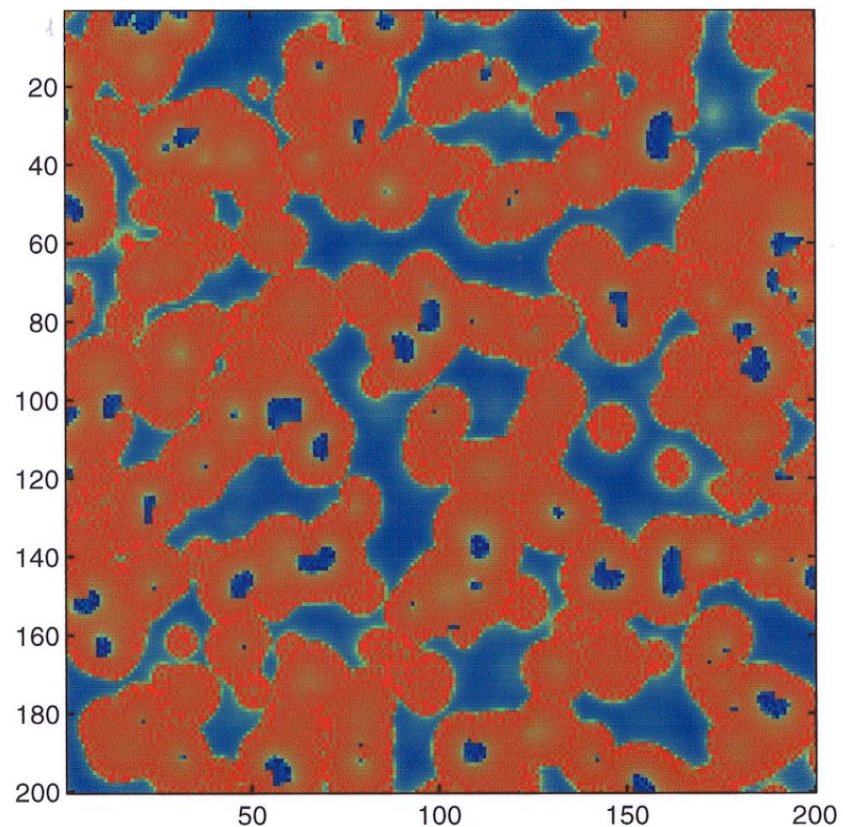

(a)

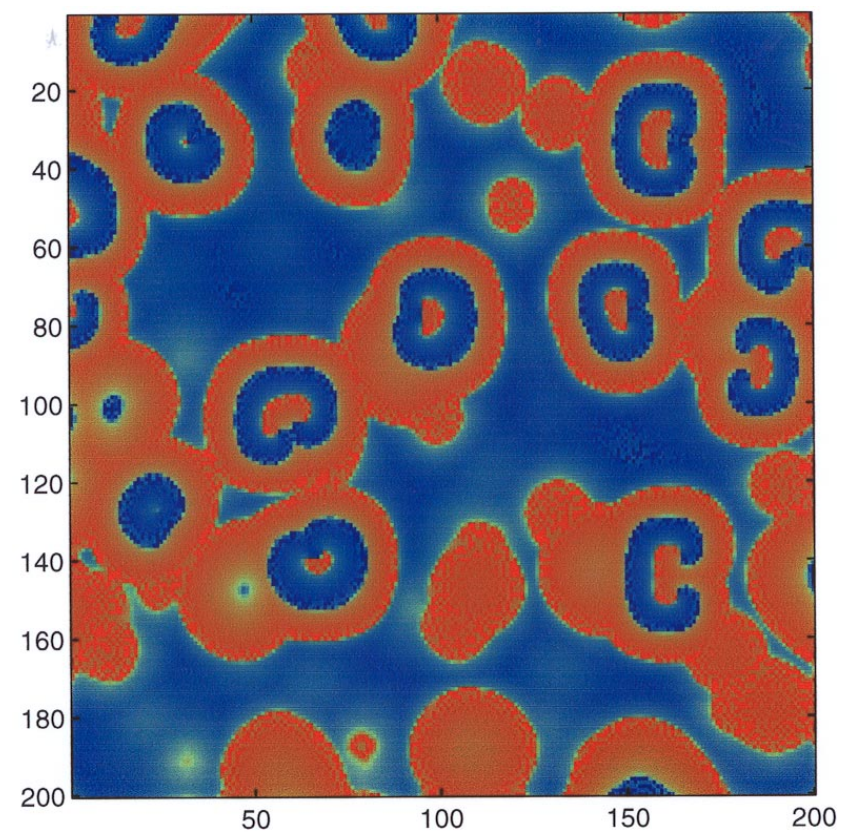

(c)

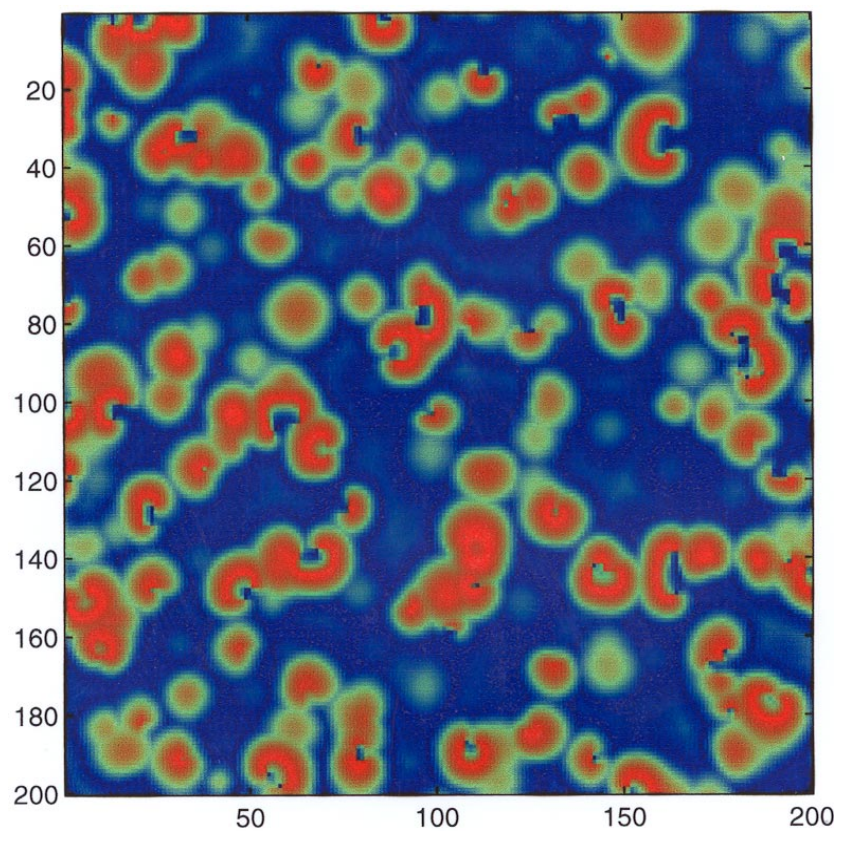

(b)

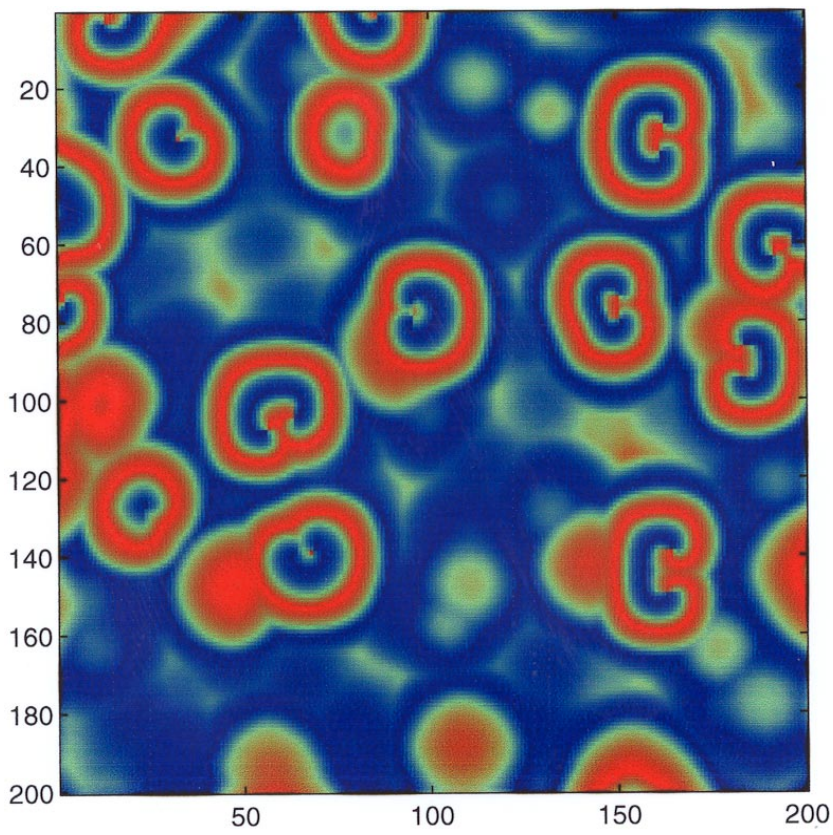

(d)

Fig. 9. Simulation results of CNN made of one-port second-order cells. The parameters are given by $\alpha=-1, b=-10$ and $D_{1}=1$. The cells are operating in the edge-of-chaos regions. (a) $V_{1}(i, j)$ at $t=50$. (b) $V_{2}(i, j)$ at $t=50$. (c) $V_{1}(i, j)$ at $t=100$. (d) $V_{2}(i, j)$ at $t=100$. (e) $V_{1}(i, j)$ at $t=200$. (f) $V_{2}(i, j)$ at $t=200$. (g) $V_{1}(i, j)$ at $t=400$. (h) $V_{2}(i, j)$ at $t=400$. 


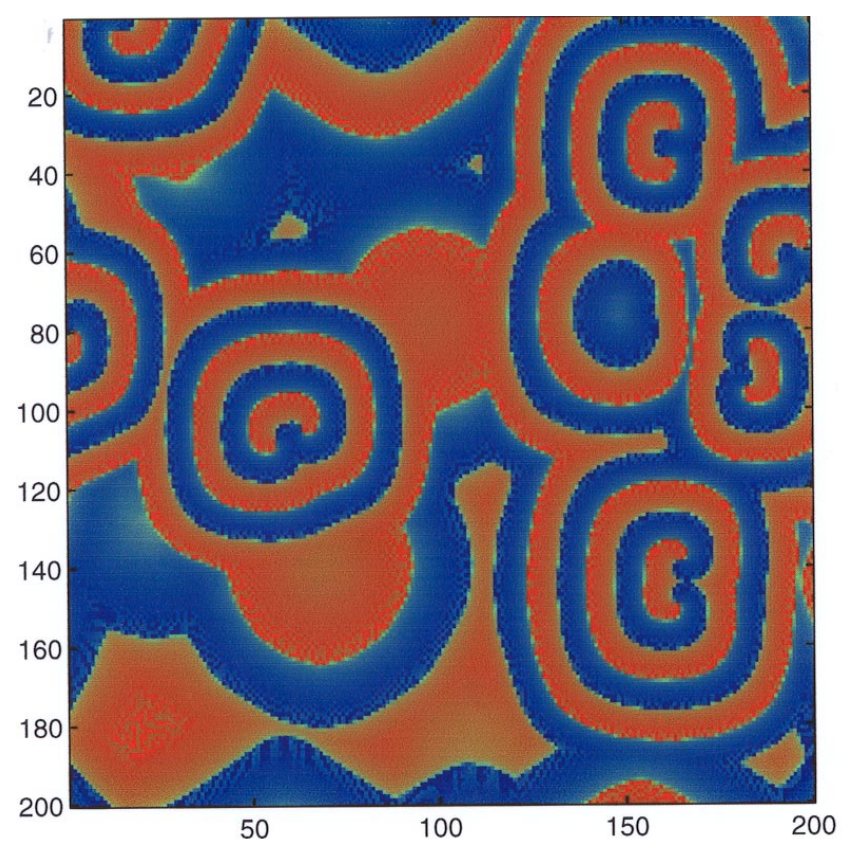

(e)

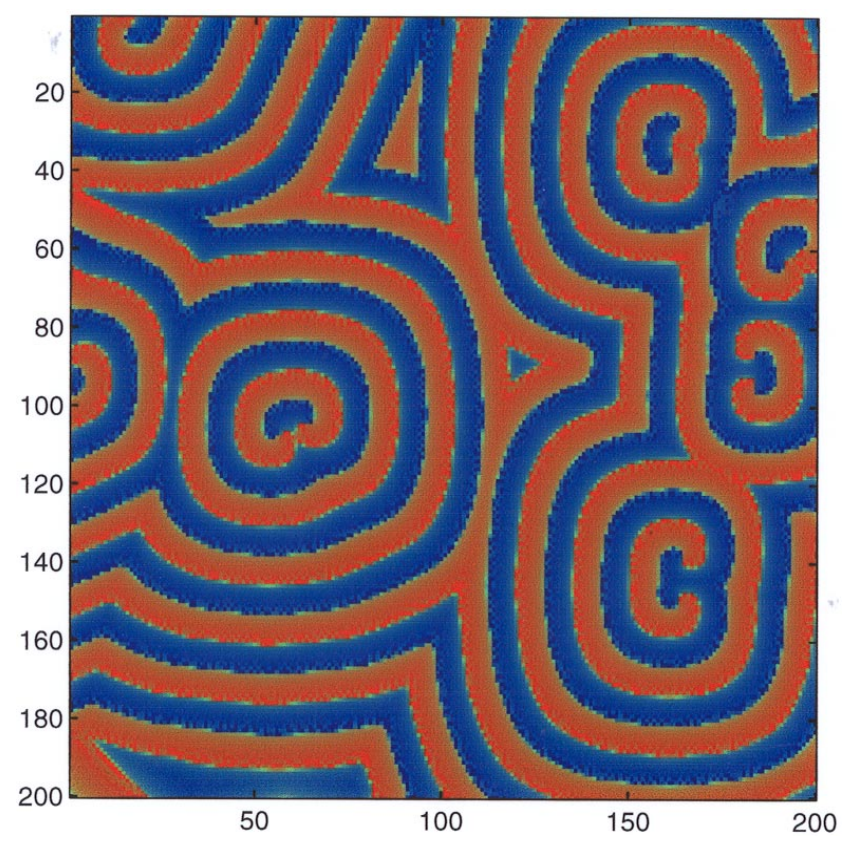

(g)

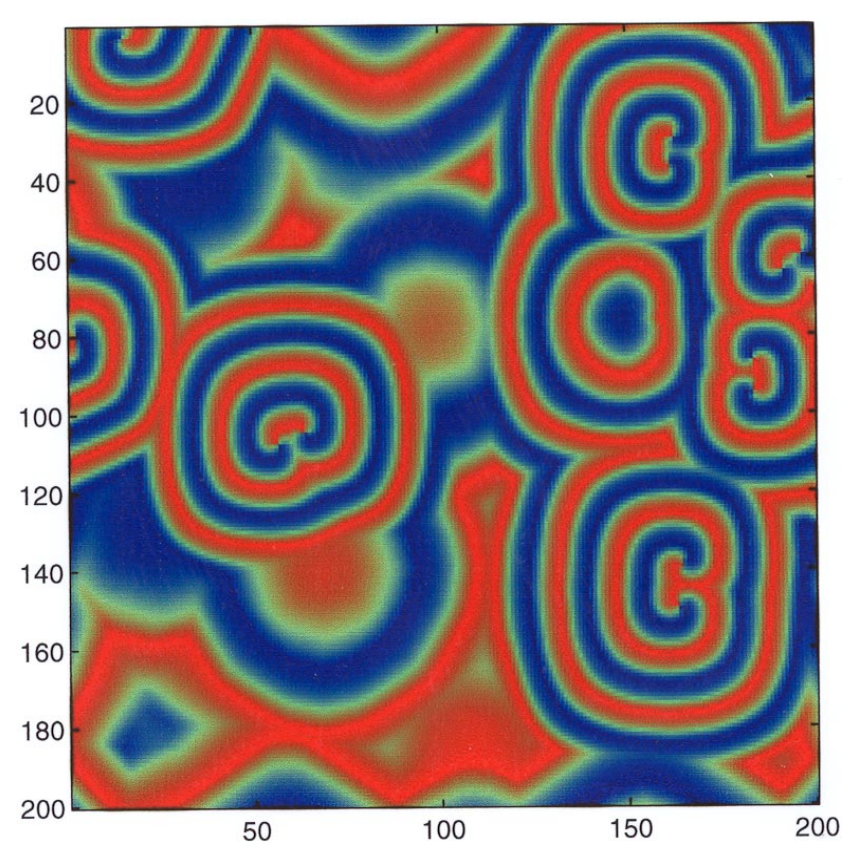

(f)

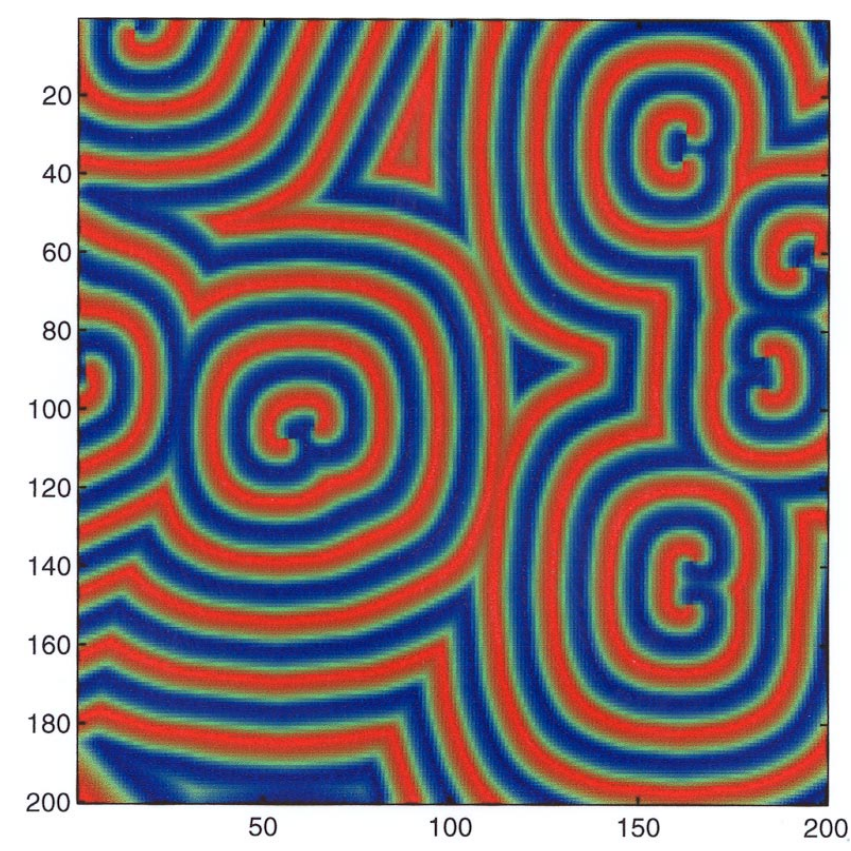

(h)

Fig. 9. (Continued) 


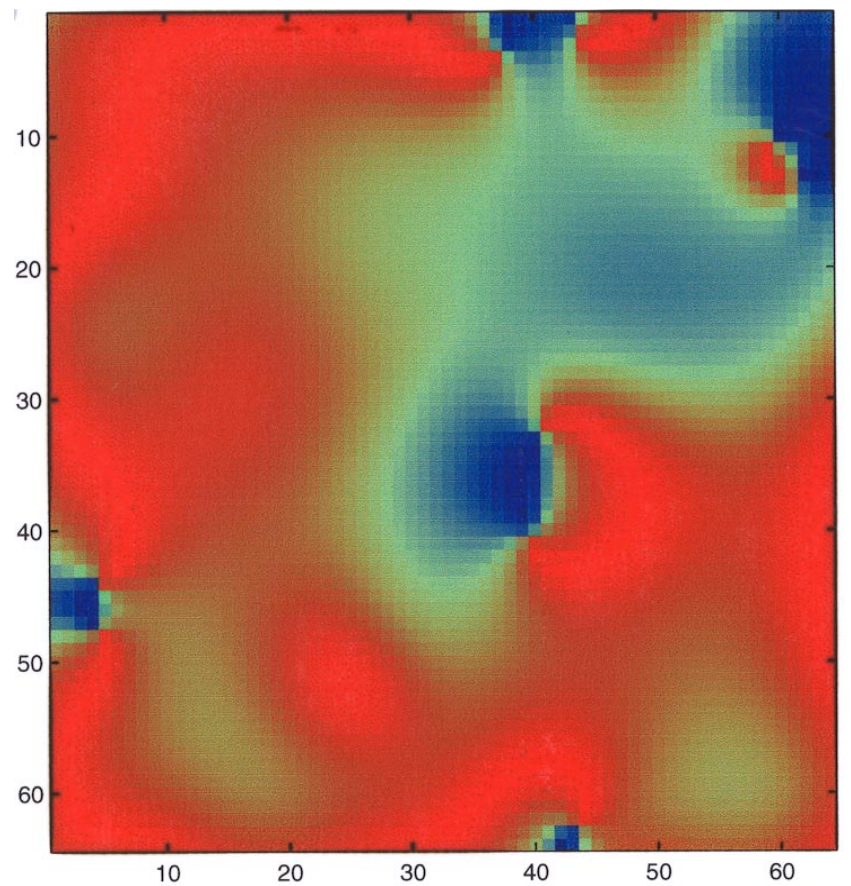

(a)

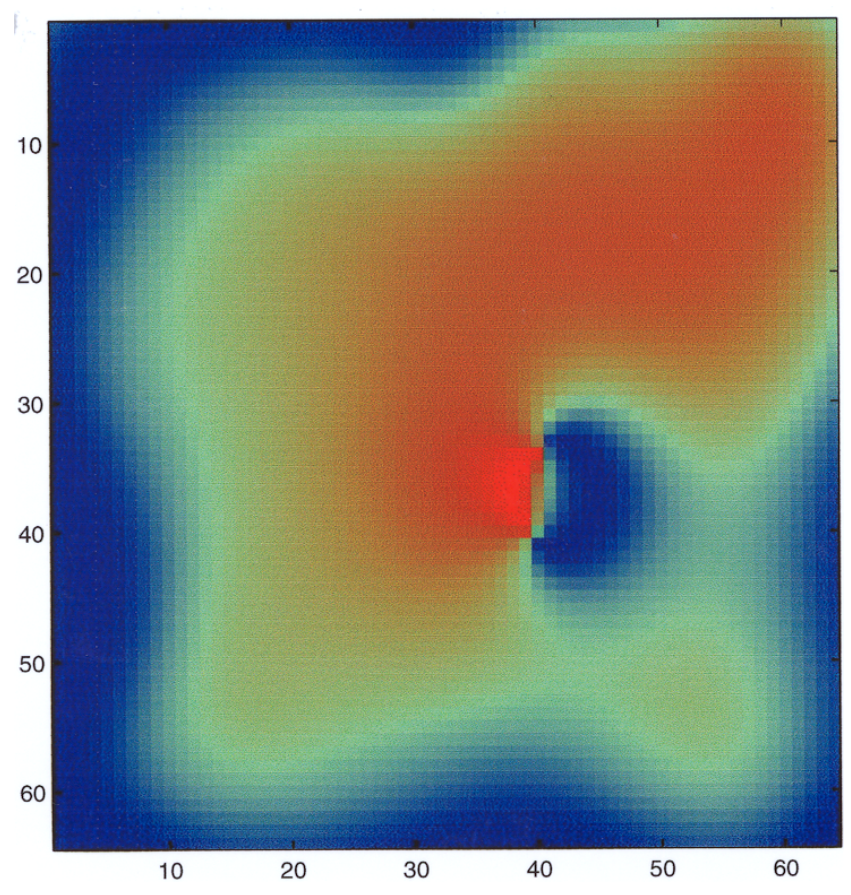

(c)

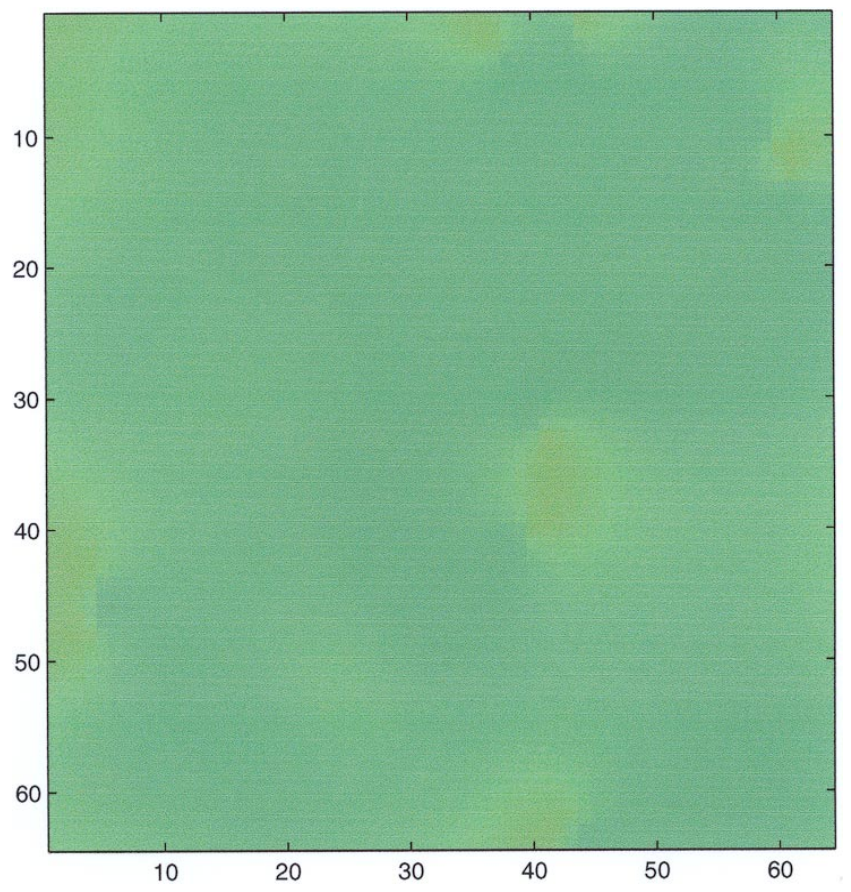

(b)

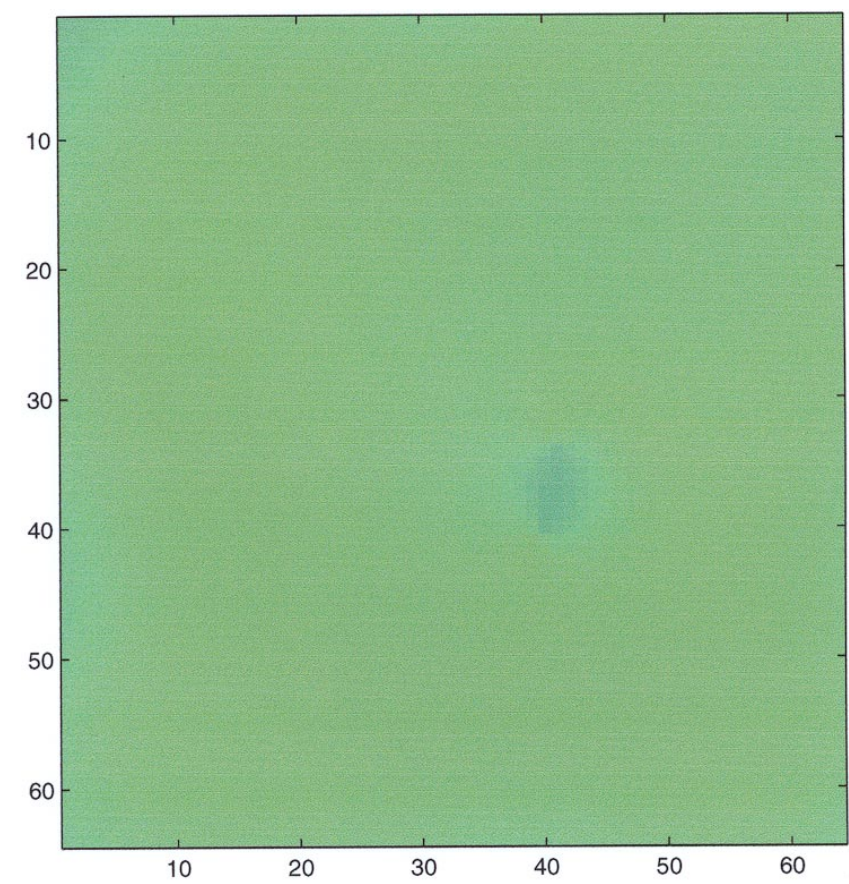

(d)

Fig. 10. Simulation results of CNN made of one-port second-order cells. The parameters are given by $\alpha=-18, b=-10$ and $D_{1}=1$. The cells are operating in the edge-of-chaos regions. (a) $V_{1}(i, j)$ at $t=50$. (b) $V_{2}(i, j)$ at $t=50$. (c) $V_{1}(i, j)$ at $t=100$. (d) $V_{2}(i, j)$ at $t=100$. (e) $V_{1}(i, j)$ at $t=200$. (f) $V_{2}(i, j)$ at $t=200$. (g) $V_{1}(i, j)$ at $t=400$. (h) $V_{2}(i, j)$ at $t=400$. (i) $V_{1}(i, j)$ at $t=450$. (j) $V_{2}(i, j)$ at $t=450$. (k) $V_{1}(i, j)$ at $t=900$. (l) $V_{2}(i, j)$ at $t=900$. (m) $V_{1}(i, j)$ at $t=1000$. (n) $V_{2}(i, j)$ at $t=1000$. (o) $V_{1}(i, j)$ at $t=2000$. (p) $V_{2}(i, j)$ at $t=2000$. 


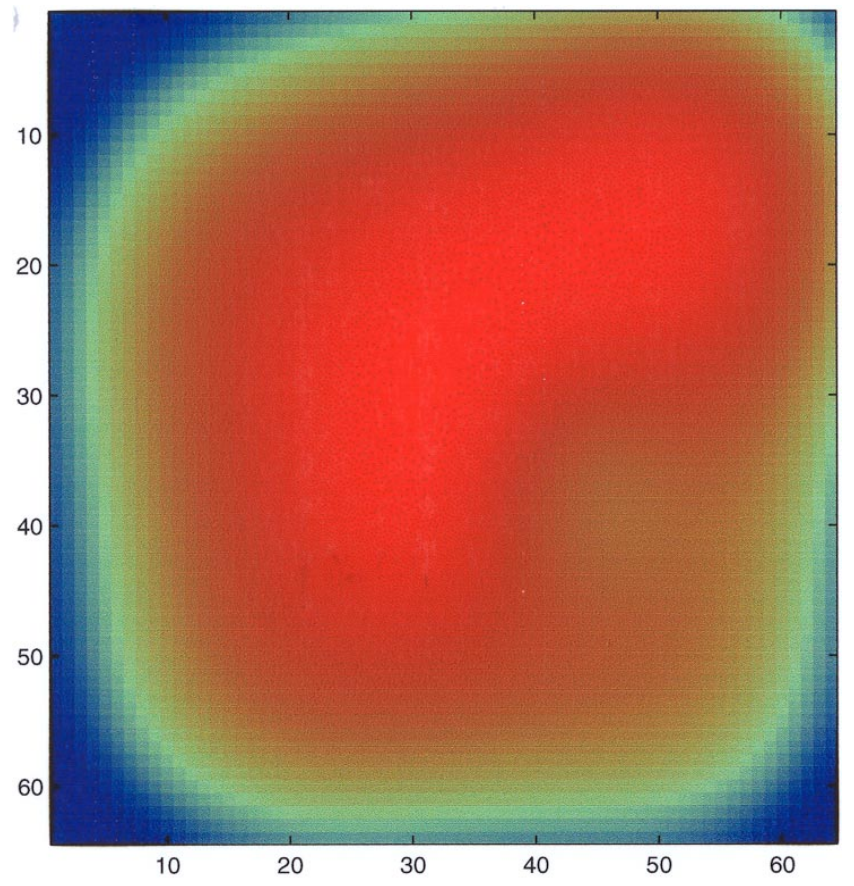

(e)

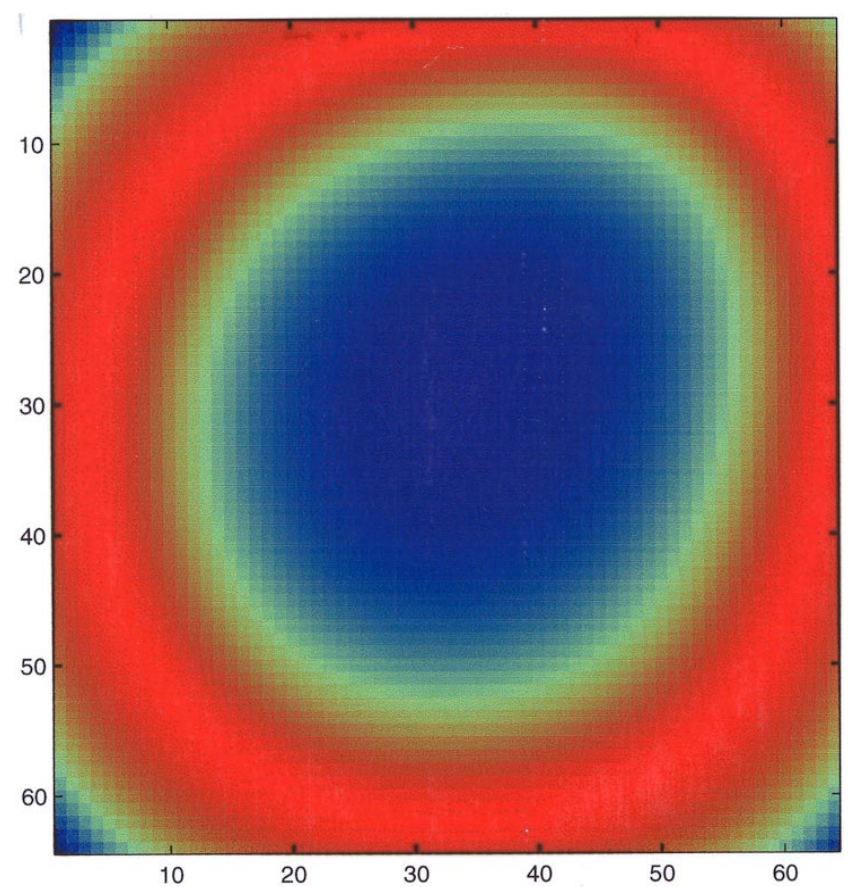

(g)

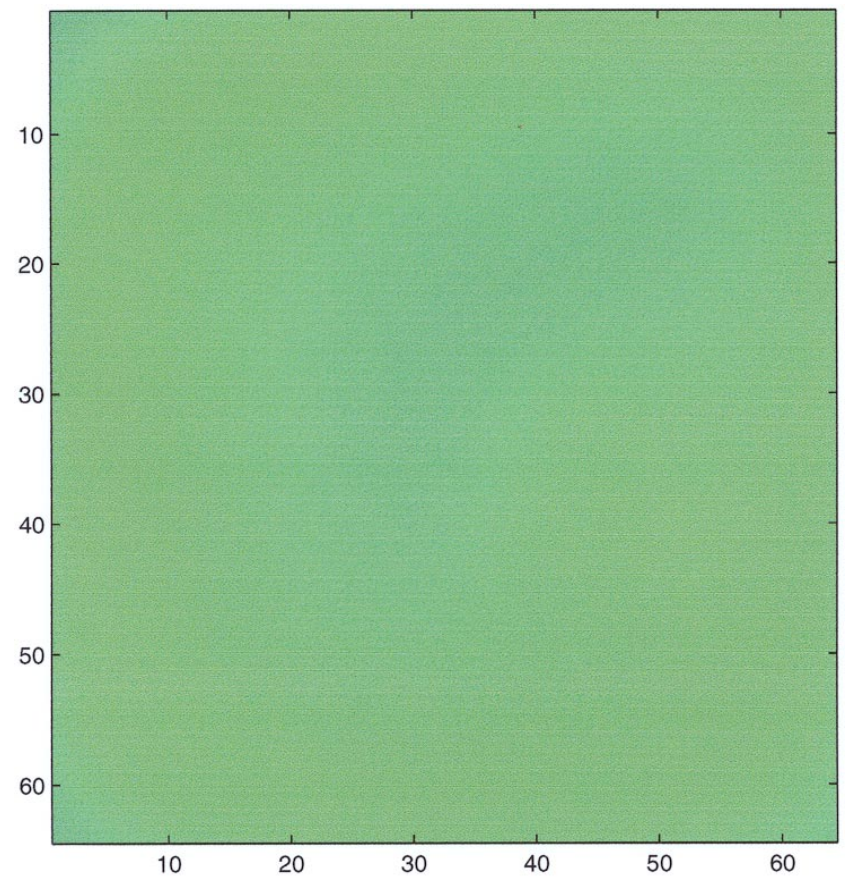

(f)

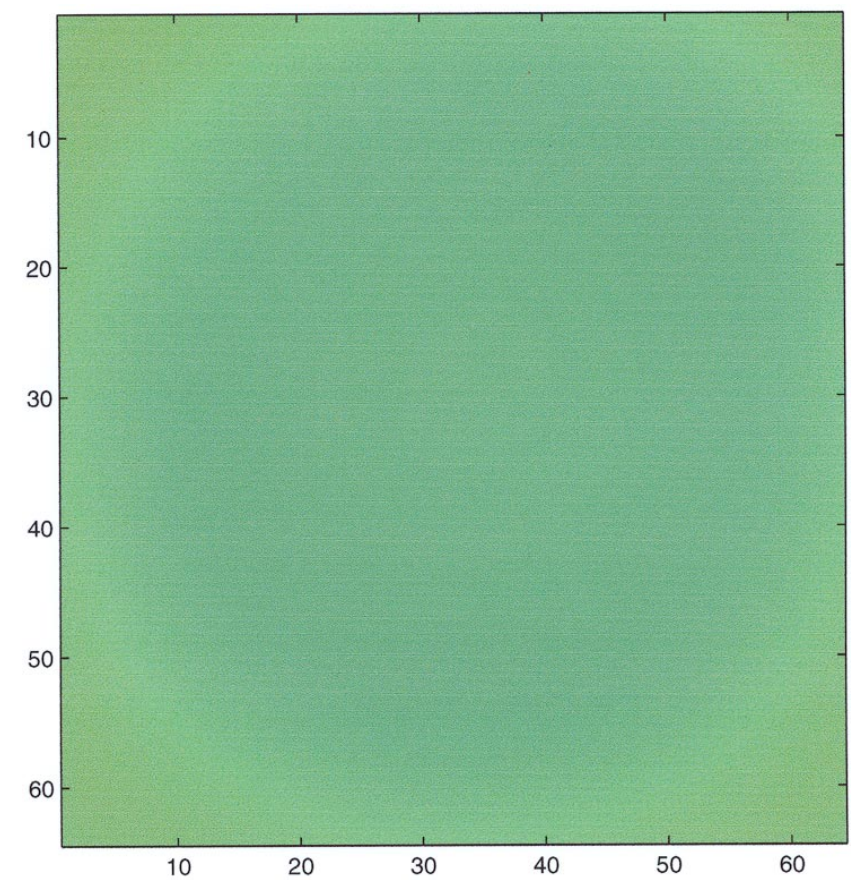

(h)

Fig. 10. (Continued) 


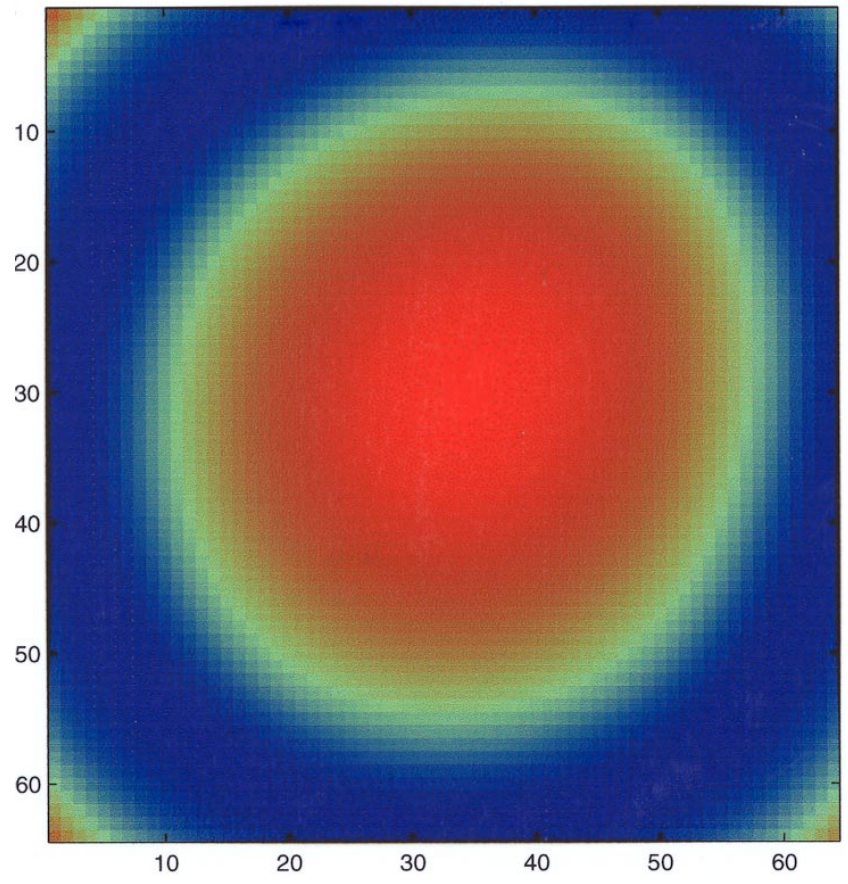

(i)

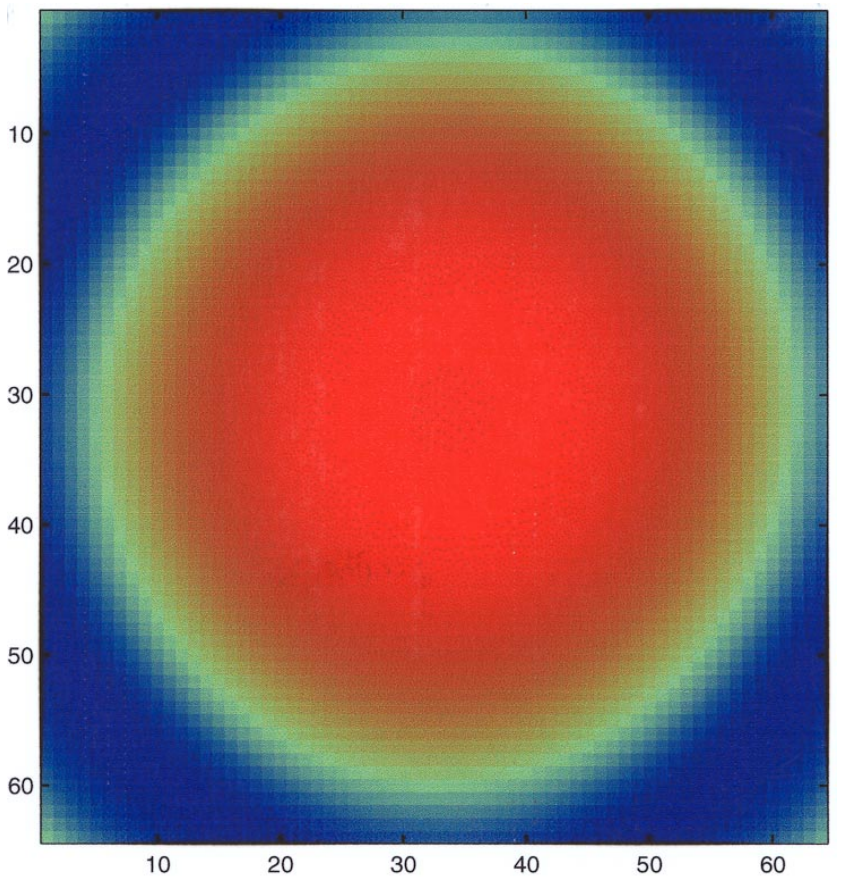

$(\mathrm{k})$

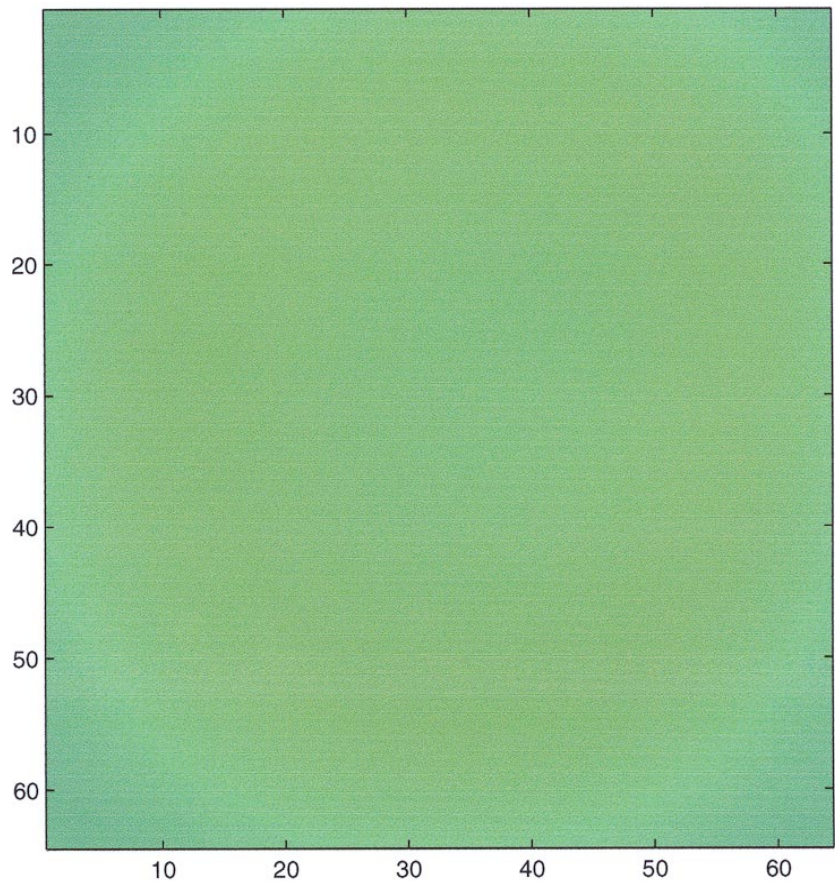

(j)

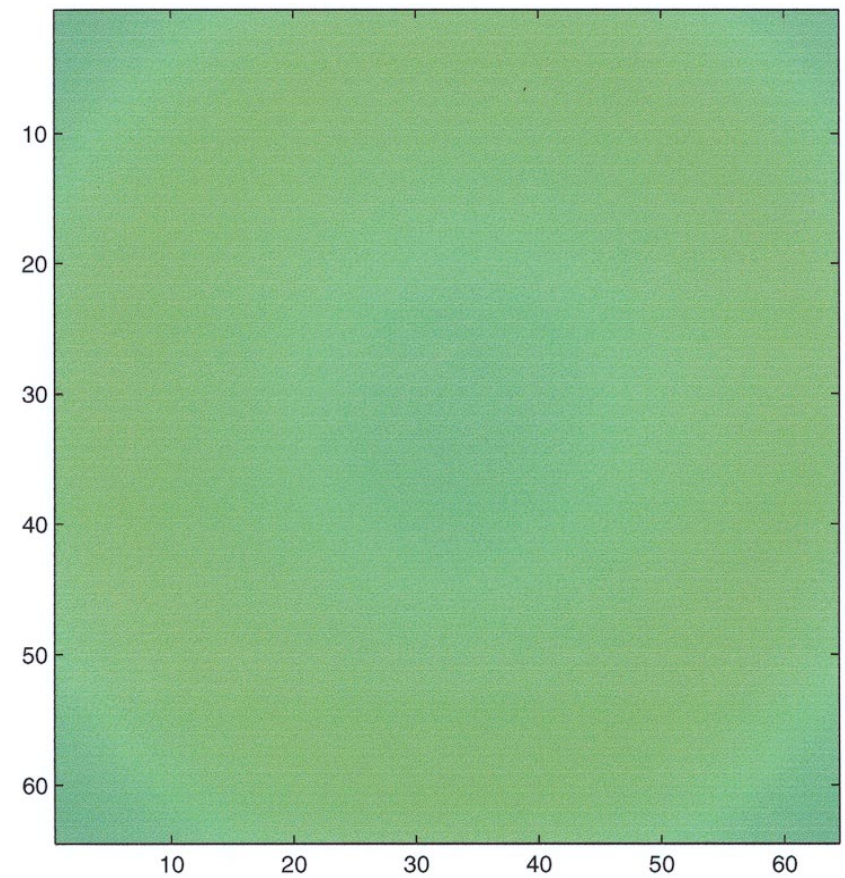

(1)

Fig. 10. (Continued) 


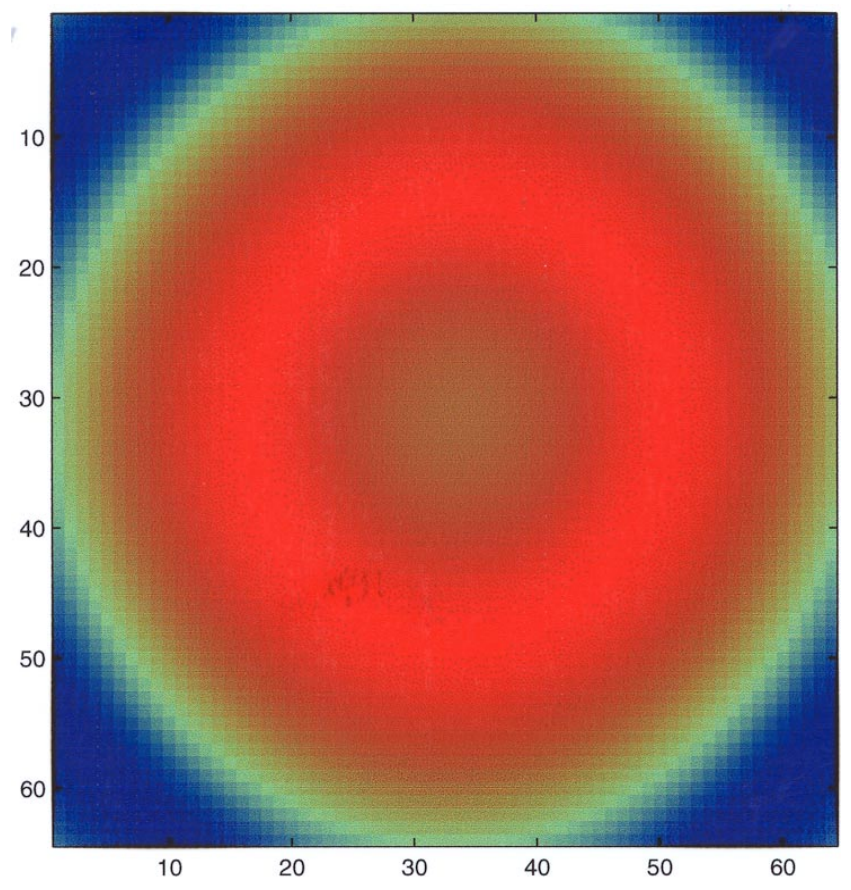

(m)

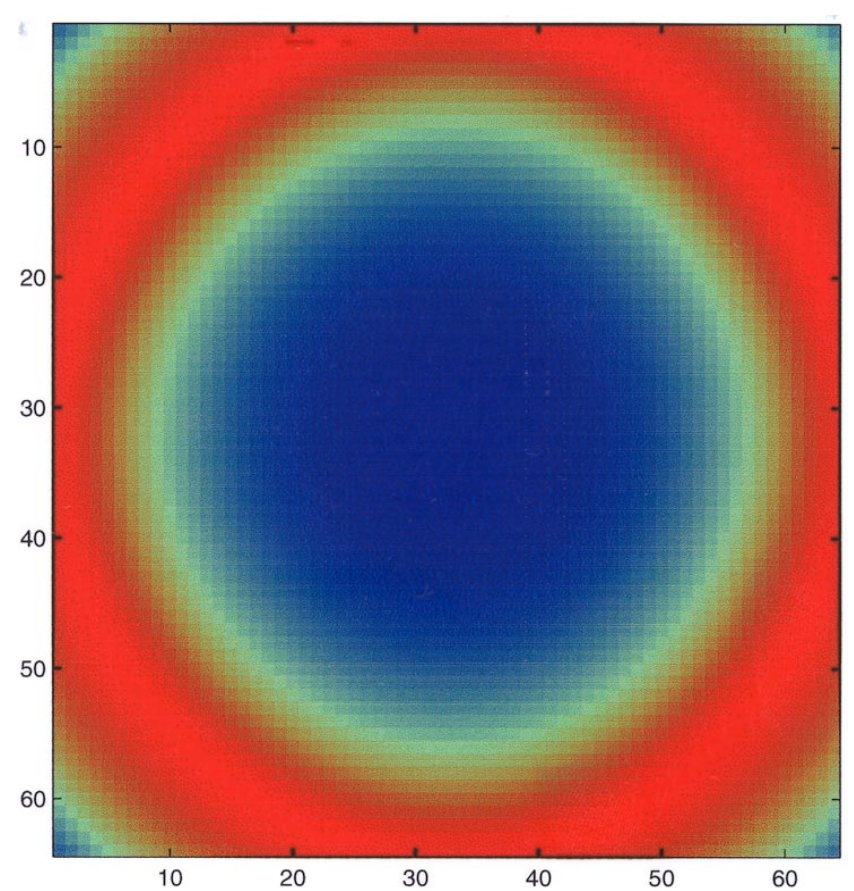

(o)

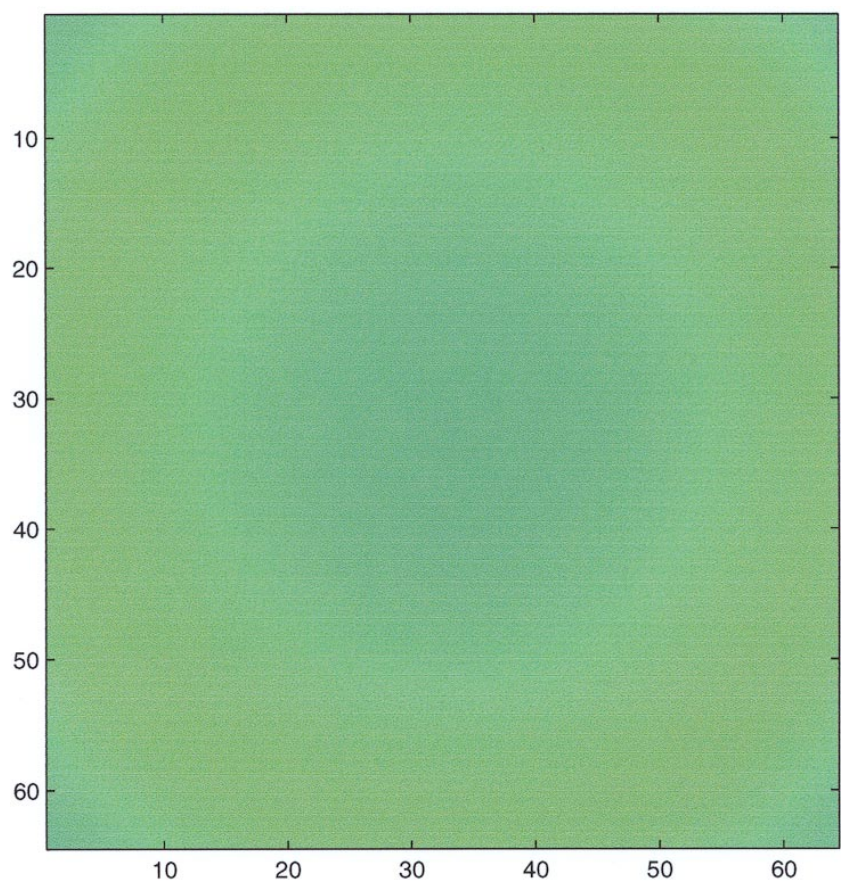

(n)

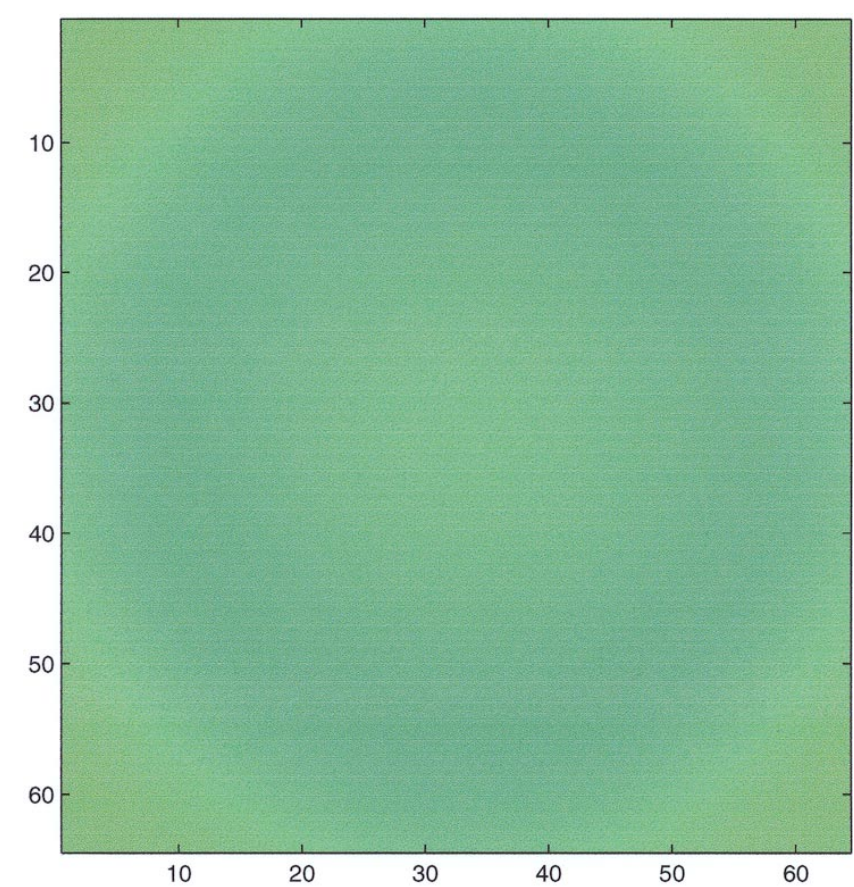

(p)

Fig. 10. (Continued) 


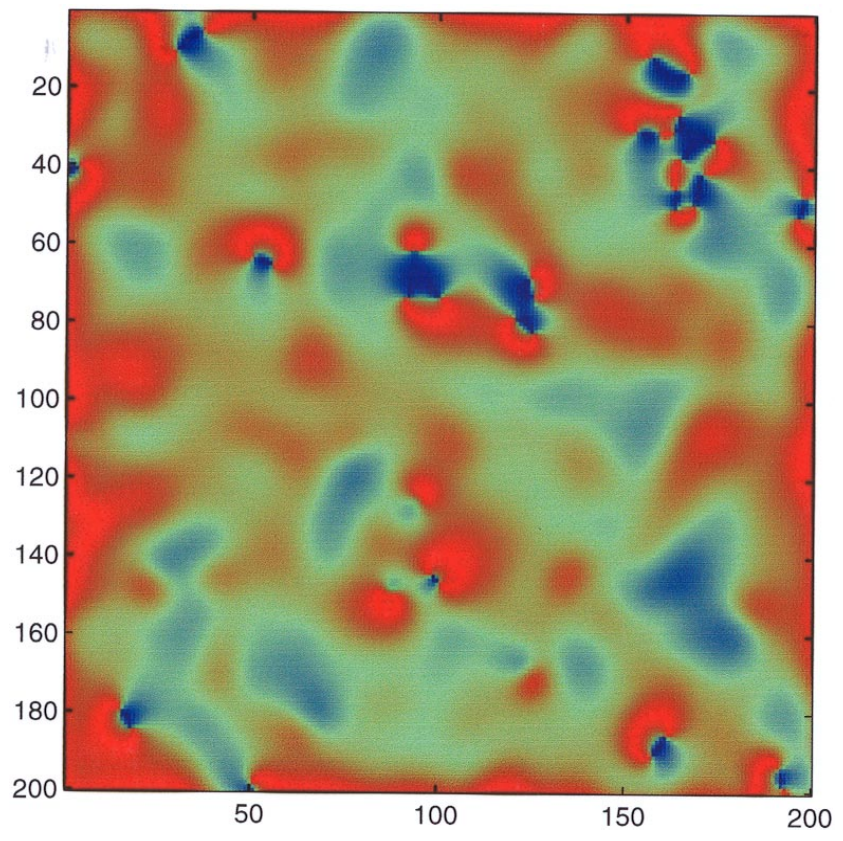

(a)

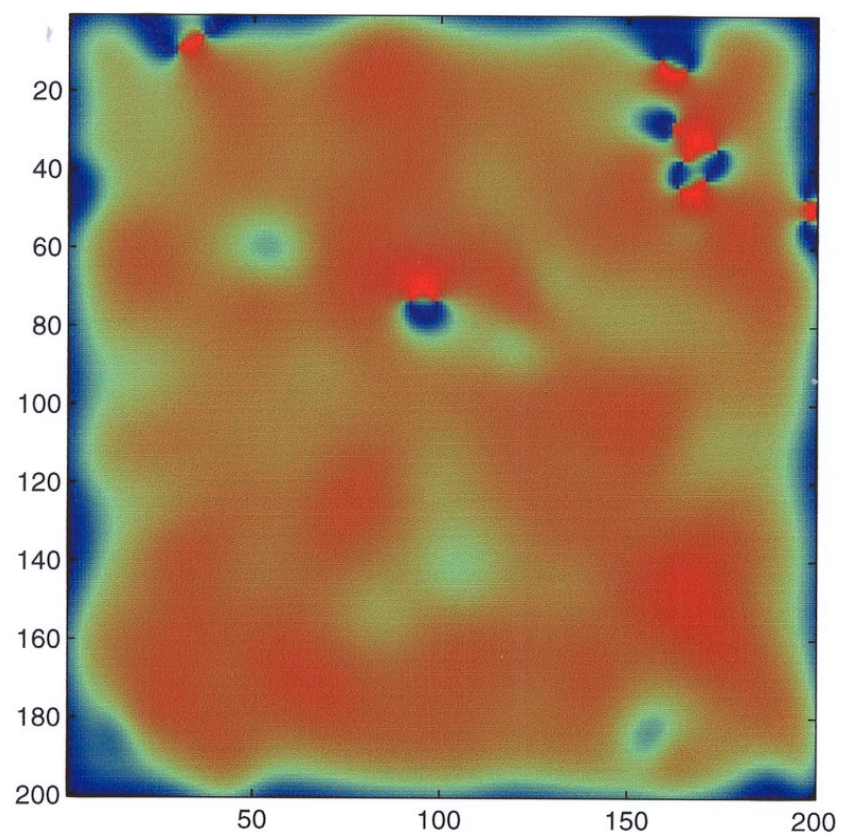

(c)

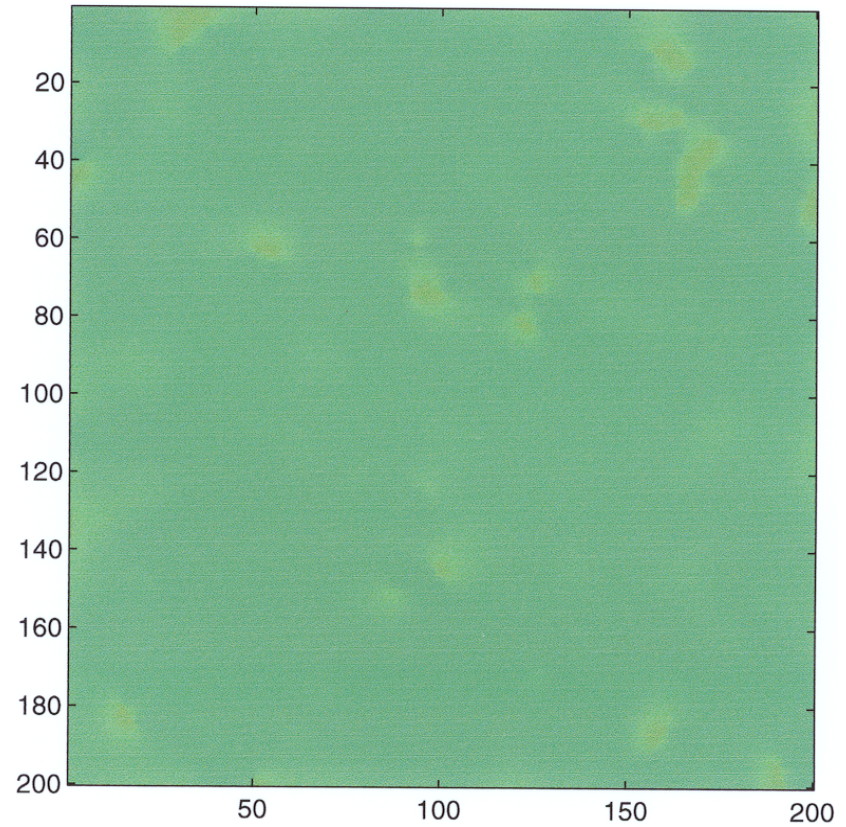

(b)

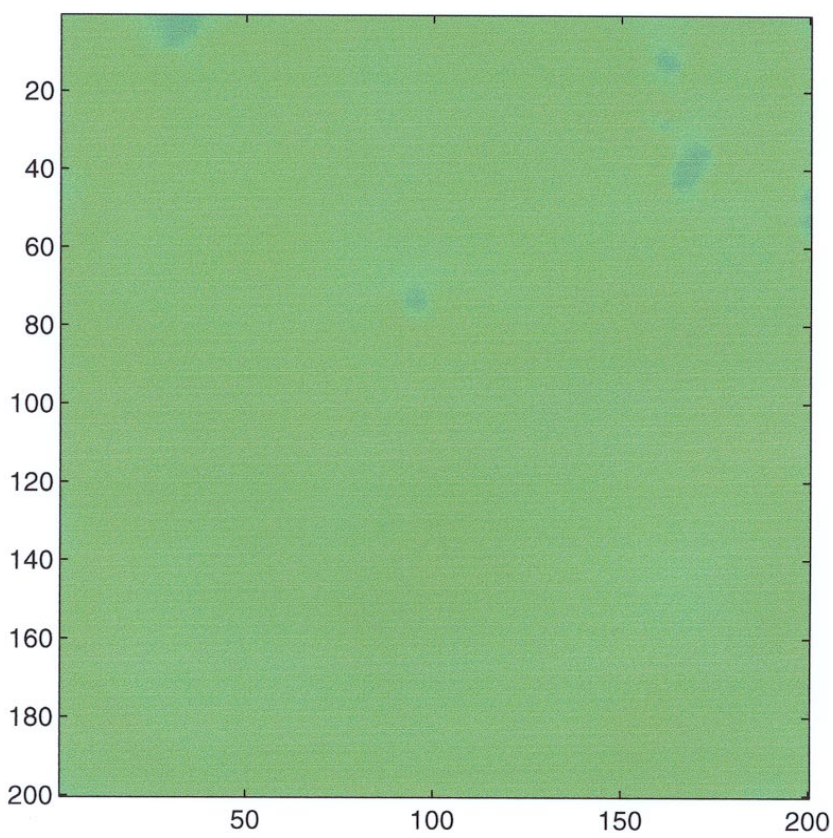

(d)

Fig. 11. Simulation results of CNN made of one-port second-order cells. The parameters are given by $\alpha=-18, b=-10$ and $D_{1}=1$. The cells are operating in the edge-of-chaos regions. (a) $V_{1}(i, j)$ at $t=50$. (b) $V_{2}(i, j)$ at $t=50$. (c) $V_{1}(i, j)$ at $t=100$. (d) $V_{2}(i, j)$ at $t=100$. (e) $V_{1}(i, j)$ at $t=300$. (f) $V_{2}(i, j)$ at $t=300$. (g) $V_{1}(i, j)$ at $t=600$. (h) $V_{2}(i, j)$ at $t=600$. 


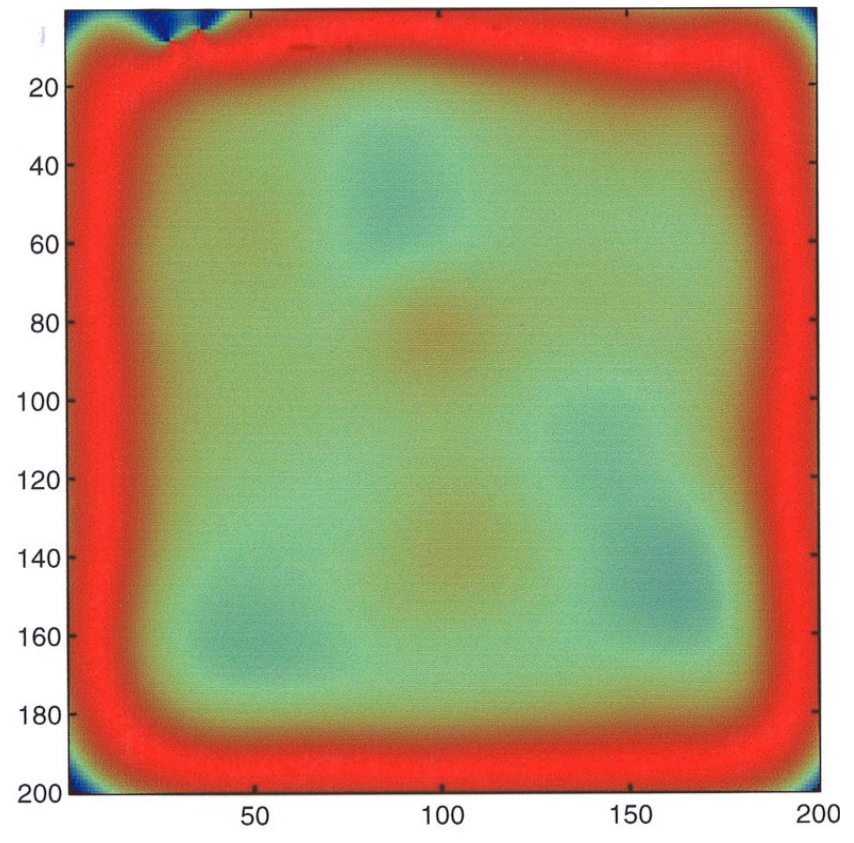

(e)

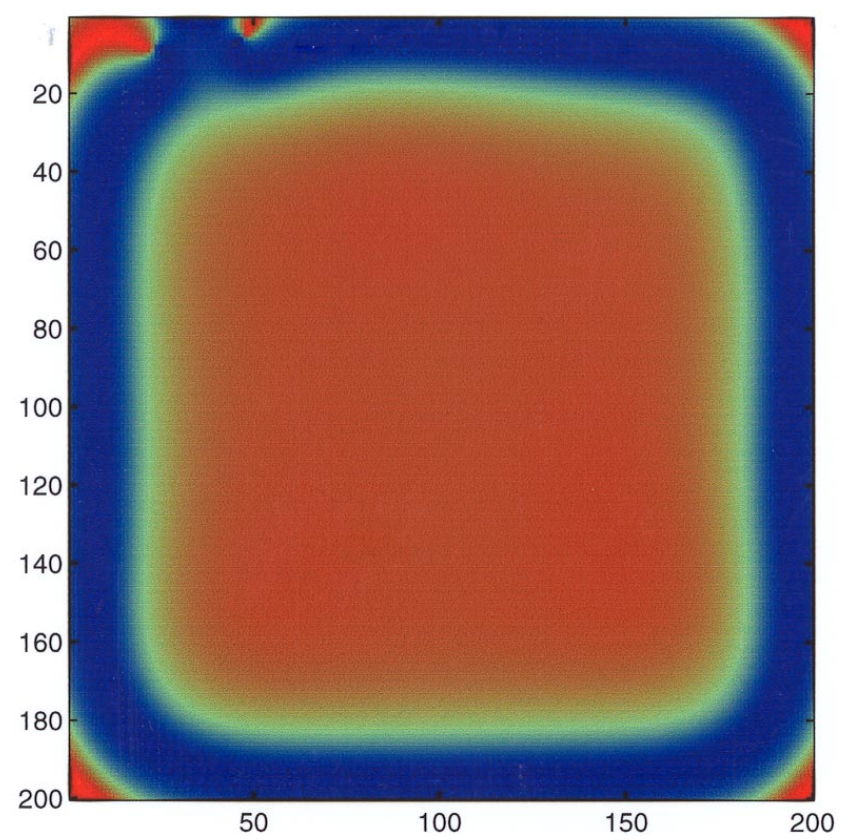

(g)

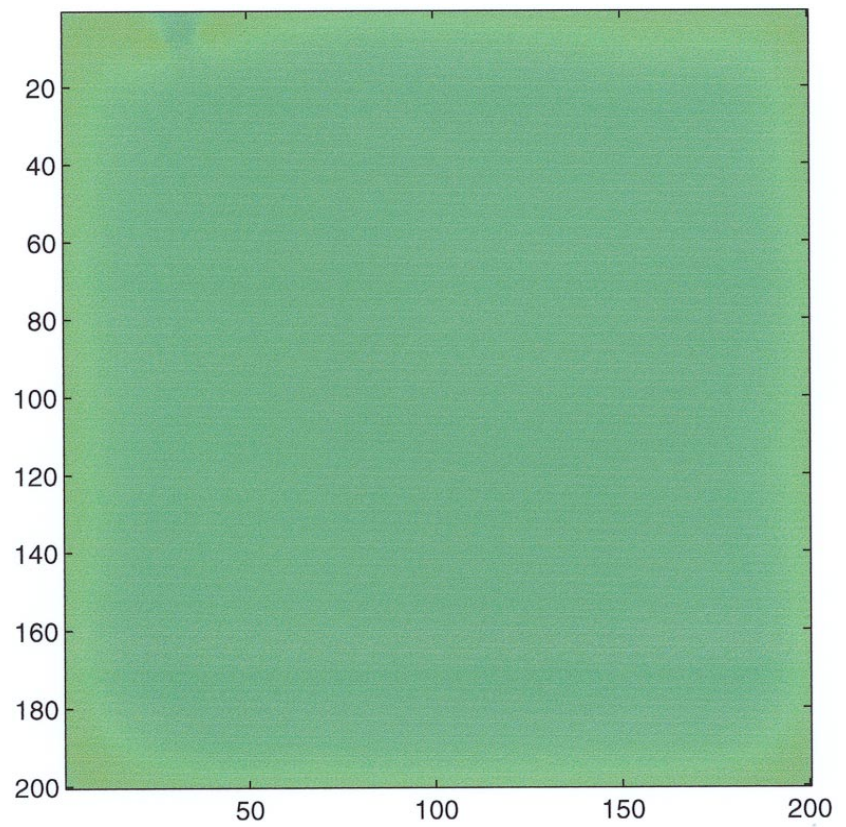

(f)

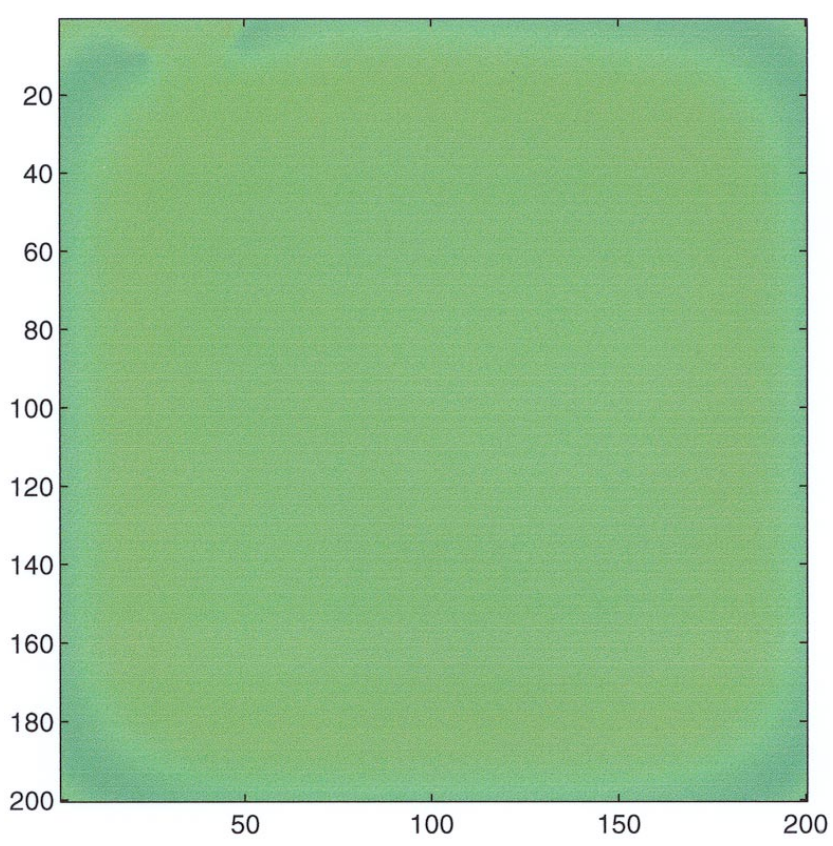

(h)

Fig. 11. (Continued) 
from which we obtain

$$
\alpha \leq 3 V_{1}^{* 2}-b+1 \quad \mathbb{A N D} \quad \alpha \leq 3 V_{1}^{* 2} .
$$

Combining the two inequalities in Eq. (157), we obtain

$$
\alpha \leq \begin{cases}3 V_{1}^{* 2}-b+1, & \text { if } b \geq 1 \\ 3 V_{1}^{* 2}, & \text { otherwise } .\end{cases}
$$

2. The parameter region defined by $\mathrm{P} 2$ is the intersection of the parameter region defined by

$$
\alpha< \begin{cases}3 V_{1}^{* 2}-b+1, & \text { if } b \geq 1, \\ 3 V_{1}^{* 2}, & \text { otherwise } .\end{cases}
$$

Hence, the parameter region satisfying $\mathrm{P} 2$ is defined by Eq. (76) or Eq. (78).

1. From P3 we have

$$
\begin{gathered}
-3 V_{1}^{* 2}+\alpha+b=0 \quad \mathbb{A N D} \quad-b=0 \quad \mathbb{A N D} \\
1=0 \quad \mathbb{A N D} \quad-1=0 .
\end{gathered}
$$

It follows that P3 leads to an empty set.

We conclude therefore that the locally passive region or the restricted locally passive region of two-port second-order CNN cells is given by

$$
\alpha< \begin{cases}3 V_{1}^{* 2}-b+1, & \text { if } b \geq 1 \\ 3 V_{1}^{* 2}, & \text { otherwise } .\end{cases}
$$

The edge-of-chaos region is empty for this case. Since the above conclusions are applicable for each equilibrium point, we need to study the behaviors of all equilibrium points in order to define the overall behaviors of the CNN cells.

\subsection{Local passive regions}

1. When $\mathfrak{D}>0$, where $\mathfrak{D}$ is as defined in Eq. (113), the local passive regions are given by

$$
\frac{I^{* 2}}{4}-\frac{\alpha^{3}}{27}>0, \quad \alpha< \begin{cases}3\left[\left(\frac{I^{*}}{2}+\sqrt{\mathfrak{D}}\right)^{1 / 3}+\left(\frac{I^{*}}{2}-\sqrt{\mathfrak{D}}\right)^{1 / 3}\right]^{2}-b+1, & \text { if } b \geq 1, \\ 3\left[\left(\frac{I^{*}}{2}+\sqrt{\mathfrak{D}}\right)^{1 / 3}+\left(\frac{I^{*}}{2}-\sqrt{\mathfrak{D}}\right)^{1 / 3}\right]^{2}, & \text { otherwise } .\end{cases}
$$

This region is identical to the intersection of the following two parameter regions:

$$
\frac{I^{* 2}}{4}-\frac{\alpha^{3}}{27}>0 \quad \mathbb{A N D} \quad \alpha<3\left[\left(\frac{I^{*}}{2}+\sqrt{\mathfrak{D}}\right)^{1 / 3}+\left(\frac{I^{*}}{2}-\sqrt{\mathfrak{D}}\right)^{1 / 3}\right]^{2}-b+1 \quad \mathbb{A N D} \quad b \geq 1
$$

and

$$
\frac{I^{* 2}}{4}-\frac{\alpha^{3}}{27}>0 \quad \mathbb{A N D} \quad b<1 .
$$

2. When $\mathfrak{D}=0$, there are two equilibrium points, and the local passive region is the intersection of the local passive parameter regions for both equilibrium points; namely,

$$
\begin{aligned}
& \frac{I^{* 2}}{4}-\frac{\alpha^{3}}{27}=0, \quad \mathbb{A N D} \quad \alpha<\left\{\begin{array}{cl}
12\left(\frac{I^{*}}{2}\right)^{2 / 3}-b+1, & \text { if } b \geq 1, \\
12\left(\frac{I^{*}}{2}\right)^{2 / 3}, & \text { otherwise. }
\end{array}\right. \\
& \alpha<\left\{\begin{array}{cl}
3\left(\frac{I^{*}}{2}\right)^{2 / 3}-b+1, & \text { if } b \geq 1, \\
3\left(\frac{I^{*}}{2}\right)^{2 / 3}, & \text { otherwise. }
\end{array}\right.
\end{aligned}
$$


This is identical to the inequalities

$$
\alpha \geq 0 \quad \mathbb{N} \mathbb{D} \quad \alpha<\left\{\begin{array}{ll}
4 \alpha-b+1, & \text { if } b \geq 1, \\
4 \alpha, & \text { otherwise. }
\end{array} \quad \mathbb{A N D} \quad \alpha< \begin{cases}\alpha-b+1, & \text { if } b \geq 1, \\
\alpha, & \text { otherwise } .\end{cases}\right.
$$

which lead to an empty set.

3. When $\mathfrak{D}<0$, there are three equilibrium points, and the local passive regions are given by the union of the local passive parameter regions at these equilibrium points; namely,

$$
\begin{gathered}
\frac{I^{* 2}}{4}-\frac{\alpha^{3}}{27}<0 \quad \mathbb{A N D} \quad \alpha< \begin{cases}3(2 \sqrt{|\alpha| / 3} \cos (\psi / 3))^{2}+b-1, & \text { if } b \geq 1, \\
3(2 \sqrt{|\alpha| / 3} \cos (\psi / 3))^{2}, & \text { otherwise } .\end{cases} \\
\alpha \leq\left\{\begin{array}{ll}
3(-2 \sqrt{|\alpha| / 3} \cos (\psi / 3-\pi / 3))^{2}+b-1, & \text { if } b \geq 1, \\
3(-2 \sqrt{|\alpha| / 3} \cos (\psi / 3-\pi / 3))^{2}, & \text { otherwise. }
\end{array} \quad\right. \text { AND } \\
\alpha \leq \begin{cases}3(-2 \sqrt{|\alpha| / 3} \cos (\psi / 3+\pi / 3))^{2}+b-1, & \text { if } b \geq 1, \\
3(-2 \sqrt{|\alpha| / 3} \cos (\psi / 3+\pi / 3))^{2}, & \text { otherwise. }\end{cases}
\end{gathered}
$$

Since these inequalities lead to an empty set, it follows that the two-port second-order CNN cell defined by Eq. (152) is always locally active (i.e. not locally passive) when $\mathfrak{D}<0$.

\subsection{Illustrative examples}

In Fig. 12 the locally passive and the locally active parameter regions are shown in the $I^{*}-\alpha$ plane. The local passive regions and the restricted local passive regions are shown in blue and cyan, respectively. There is no edge-of-chaos region. The locally active unstable regions are shown in green. Figures 12(a)12 (c) show the cases when $b=1, b=10$ and $b=20$, respectively.

We will now present our simulation results for an $N \times N$ CNN described by the following equations:

$$
\begin{aligned}
\dot{V}_{1}(i, j)= & -V_{1}^{3}(i, j)+(\alpha+b) V_{1}(i, j)-b V_{2}(i, j) \\
& +D_{1}\left[V_{1}(i-1, j)+V_{1}(i, j-1)\right. \\
& \left.+V_{1}(i, j+1)+V_{1}(i+1, j)-4 V_{1}(i, j)\right] \\
\dot{V}_{2}(i, j)= & V_{1}(i, j)-V_{2}(i, j),+D_{2}\left[V_{2}(i-1, j)\right. \\
& +V_{2}(i, j-1)+V_{2}(i, j+1) \\
& \left.+V_{2}(i+1, j)-4 V_{2}(i, j)\right] \\
& 1 \leq i \leq N, 1 \leq j \leq N
\end{aligned}
$$

For all simulations presented in this section, the random initial values for $V_{1}(i, j)$ and $V_{2}(i, j)$ are uniformly distributed over the unit interval $(0,1)$.

The simulation results with parameters $\alpha=$ $-1, b=1, D_{1}=1$ and $D_{2}=1$ are shown in Fig. 13 . As shown in Fig. 12(a), this CNN is locally passive. Therefore, for any initial condition this CNN will eventually settle down to the same homogeneous patterns as shown in Fig. 13(d).

The simulation results with parameters $\alpha=2$, $b=1, D_{1}=1$ and $D_{2}=1$ are shown in Fig. 14. As shown in Fig. 12(a), this CNN is operating in the restricted locally active parameter region. Observe that this CNN converges to a stationary pattern as shown in Fig. 14(f).

Our simulation results with parameters $\alpha=$ $-1, b=10, D_{1}=1$ and $D_{2}=1$ are shown in Fig. 15. As shown in Fig. 12(b), this CNN is operating in the restricted locally active parameter region. Observe that at the beginning many small spiral waves emerge from the random initial condition. Subsequently, these small spiral waves then merge into a few bigger spiral waves. Towards the end the pattern evolves into a single "square" target wave which dominated the entire CNN. However, since the characteristic feature size ${ }^{4}$ in this example is bigger than the CNN array size, not all features of pattern formation from this $\mathrm{CNN}$ can be observed with only a $64 \times 64$ array size. Therefore, the dominant "square" target wave should be

\footnotetext{
${ }^{4}$ We can think of this as a characteristic spatial frequency of the resulting pattern.
} 
(a)

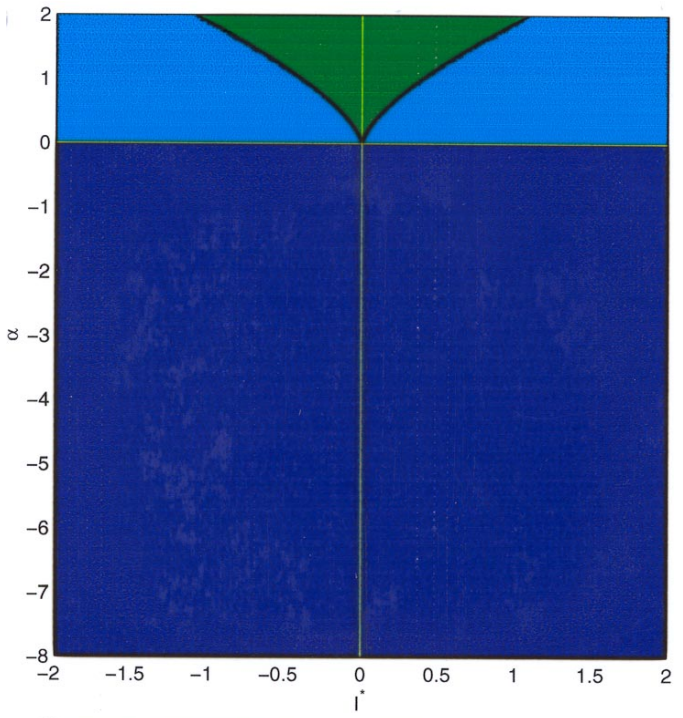

(b)

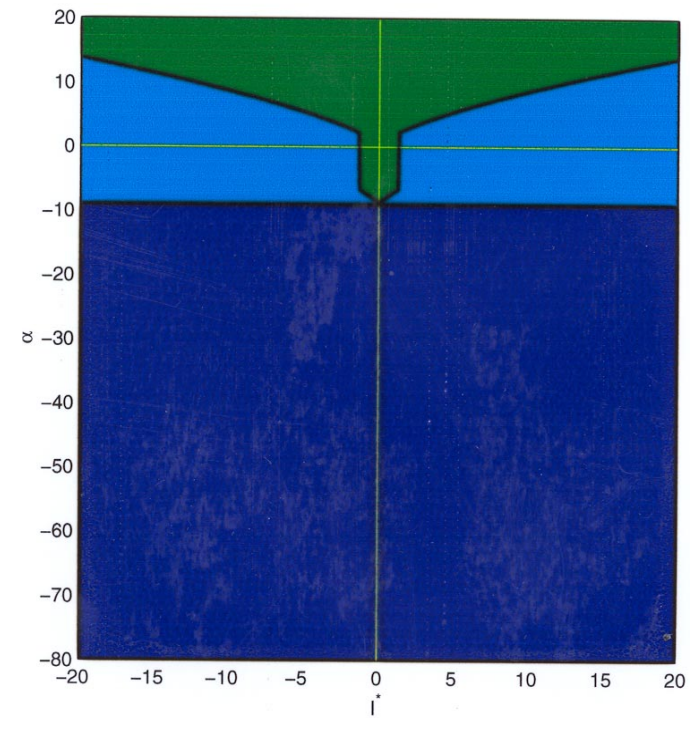

(c)

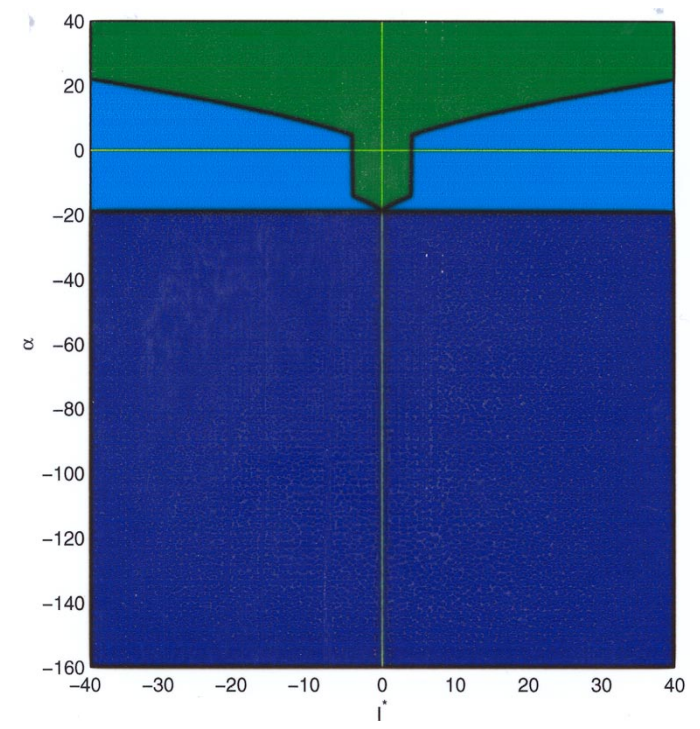

viewed only as a subset of spatial features that this pattern formation process can generate. But it is worthwhile to study small-size CNN arrays because our results show that in a spiral wave formation process, the target wave pattern can be stable in local regions. To give a more "global" view of the pattern-formation properties of this CNN, we simulate it with a $200 \times 200$ array as shown in Fig. 16 . Observe that in this larger global scale, the pattern formation process is a kind of spiral wave process, but in the local regions, we see the strip patterns that contribute to the "square" target wave depicted earlier in Fig. 15.

\section{Two-Port and Third-Order CNN Cells}

Let us consider next two-port CNN cells described by third-order state equations. Unlike the scalar one-port CNN cells studied in Sec. 2, we must now work with matrix functions of the complex variable $s$ since the number of ports " $n$ " is greater than one. The algebra in such cases can become excessive and unmanageable. Moreover, one quickly loses intuition even if explicit algebraic expressions may be derived with the help of computer symbolic softwares for certain examples. Much unwieldy algebra can be avoided, however, by exploiting the classic circuit-theoretic result which asserts that a linear $n$-port characterized by an $n \times n$ impedance matrix $\mathbf{Z}(s)$ is passive if, and only if, its associated $a d$ mittance matrix $\mathbf{Y}(s)$ is passive. We have already given an elementary and self-contained proof of this fundamental result for the scalar case in Sec. 2. A rigorous proof for the $n$-port case where $n>1$ is unfortunately much more involved [Brayton et al., 1978]. Applying this result, we can formulate the following effective approach ${ }^{5}$ for deriving the local activity parameter region for two-port third-order CNN cells.

Fig. 12. Parameter regions for two-port second-order CNN cells. The locally passive regions and restricted locally passive regions are shown in blue and cyan, respectively. The locally active unstable regions are shown in green. There is no edge-of-chaos region. (a) $b=1$. (b) $b=10$. (c) $b=20$.

\footnotetext{
${ }^{5}$ This approach obviates the extremely messy task of finding the inverse of a matrix function of the complex variable $s$.
} 


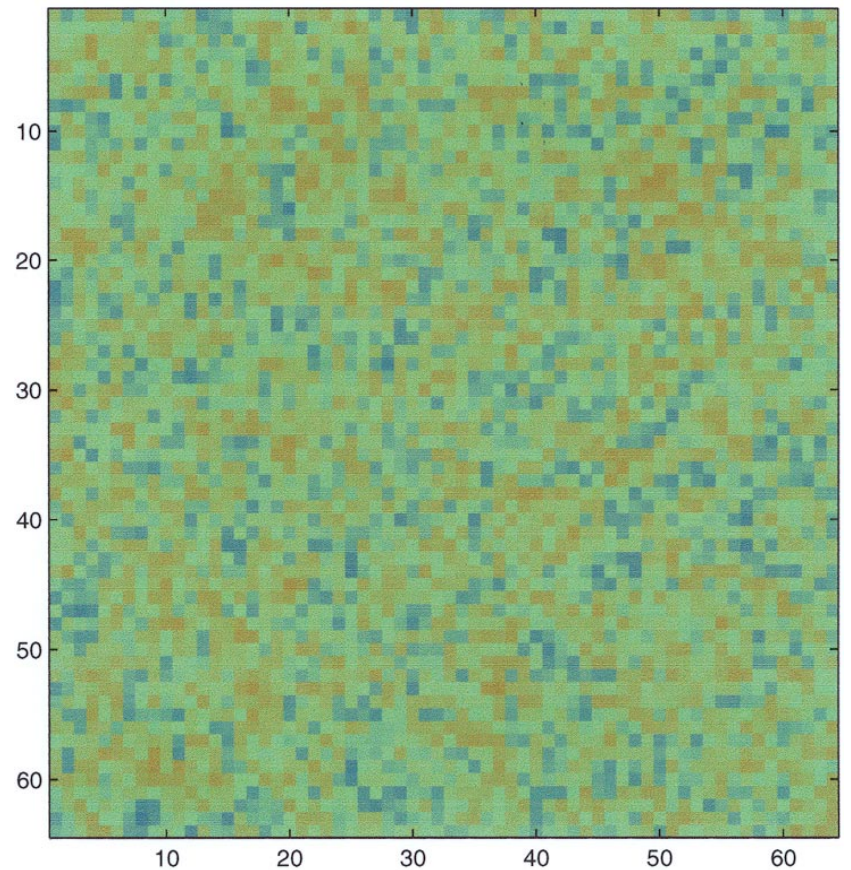

(a)

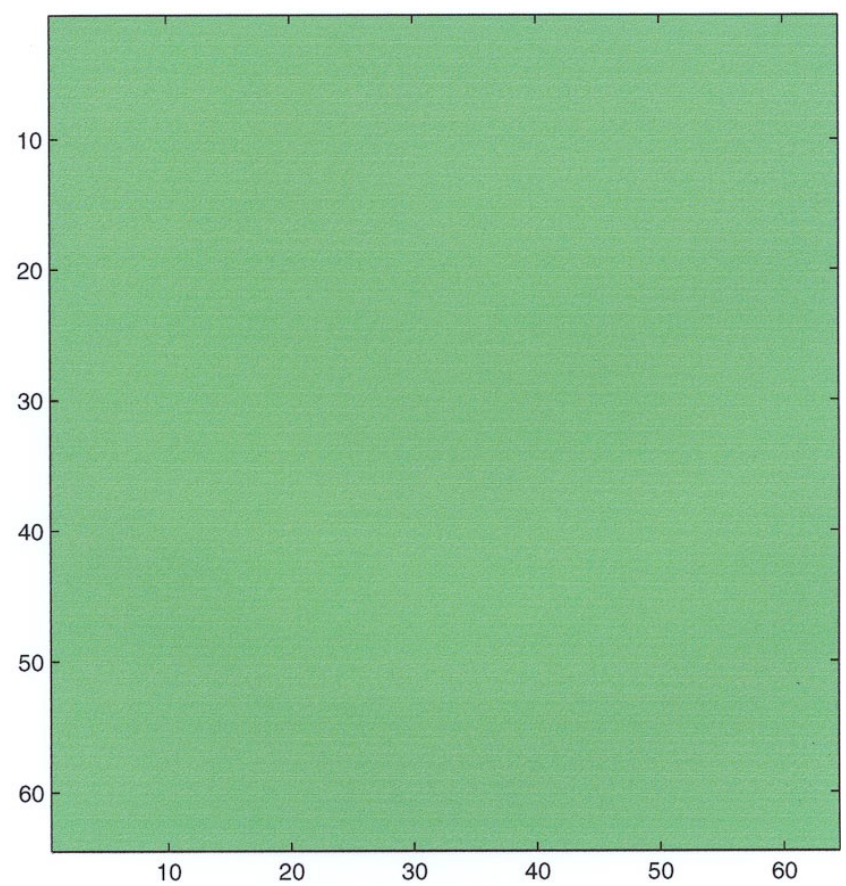

(c)

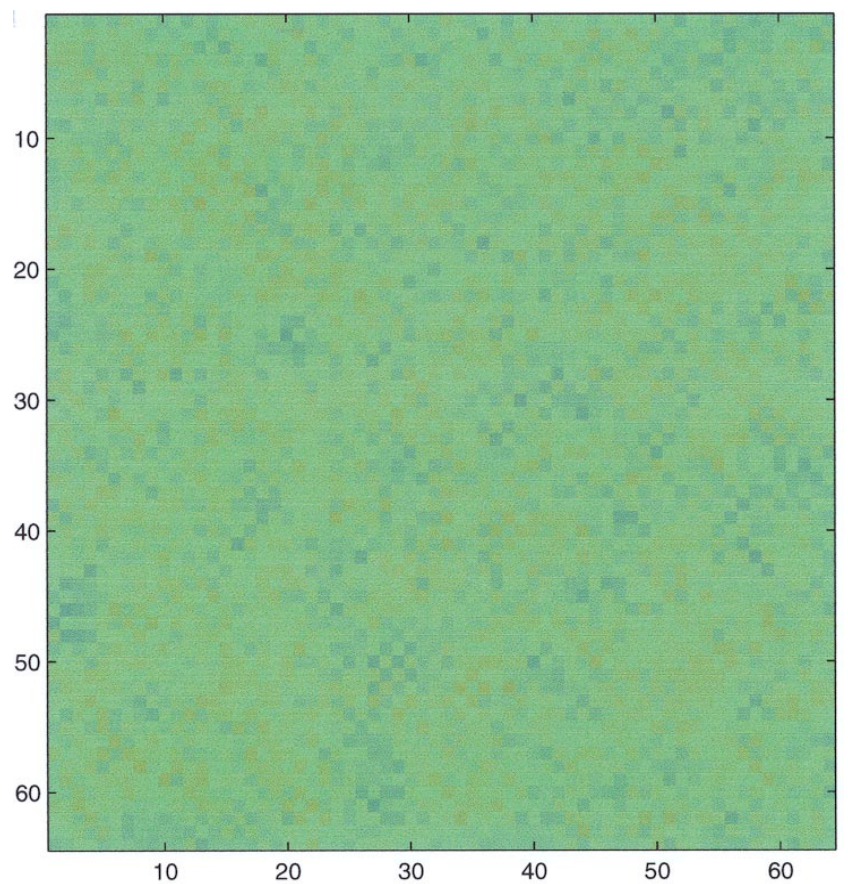

(b)

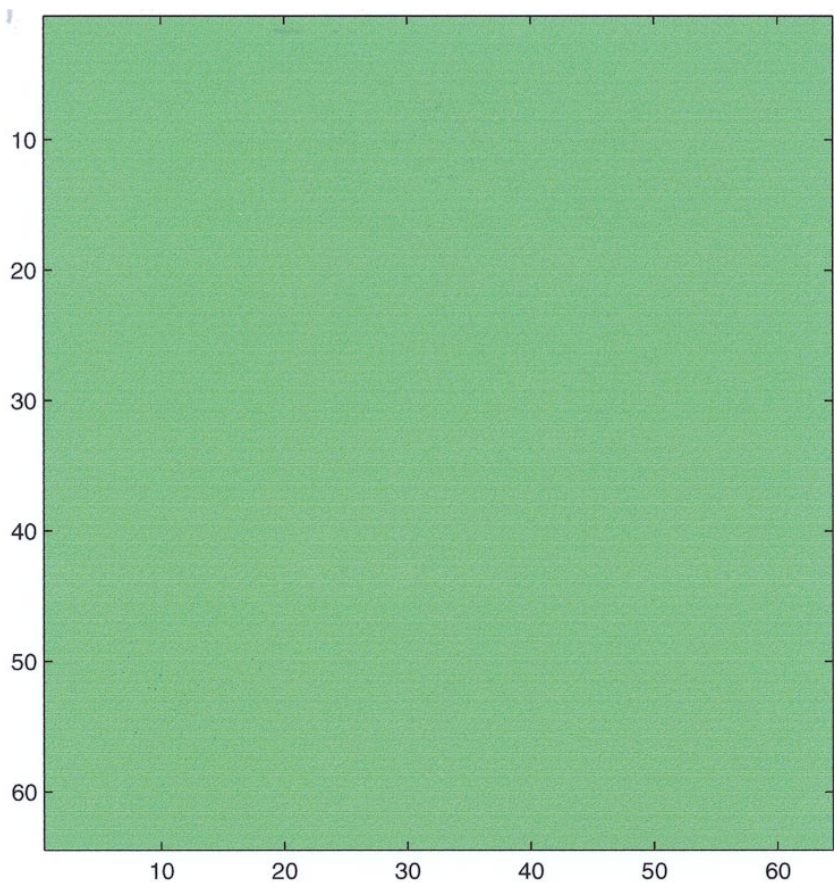

(d)

Fig. 13. Simulation results of a $64 \times 64 \mathrm{CNN}$ made of two-port second-order cells, where $\alpha=-1, b=1, D_{1}=1$ and $D_{2}=1$ are fixed. This CNN is locally passive. (a) $V_{1}(i, j)$ at $t=1$. (b) $V_{2}(i, j)$ at $t=1$. (c) $V_{1}(i, j)$ at $t=10$. (d) $V_{2}(i, j)$ at $t=10$. 


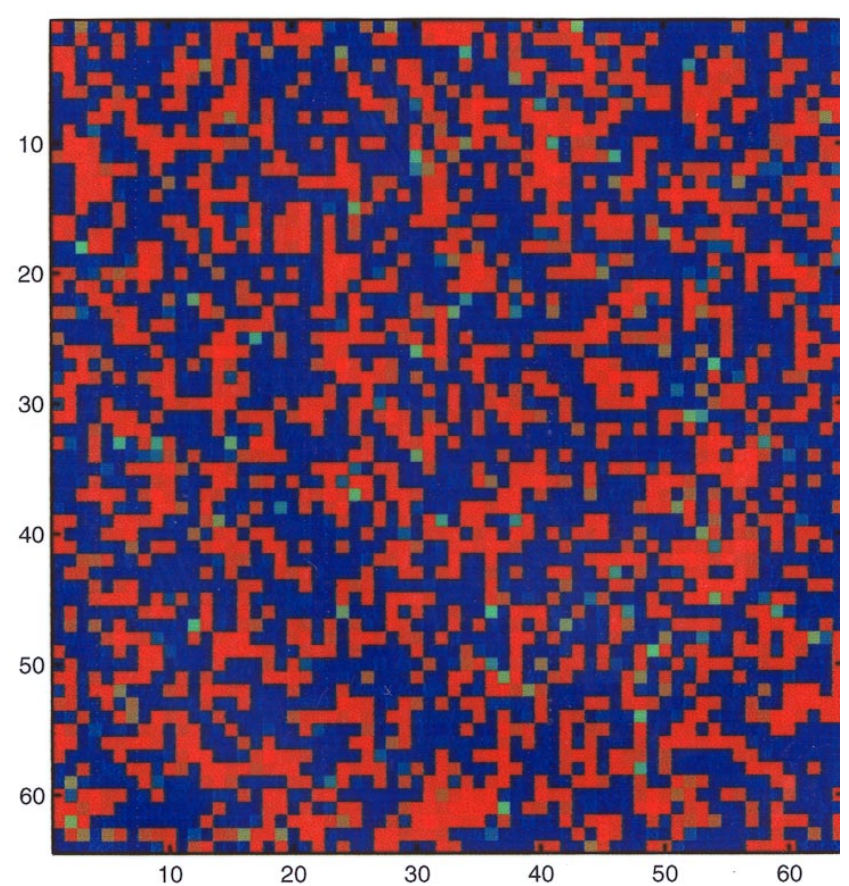

(a)

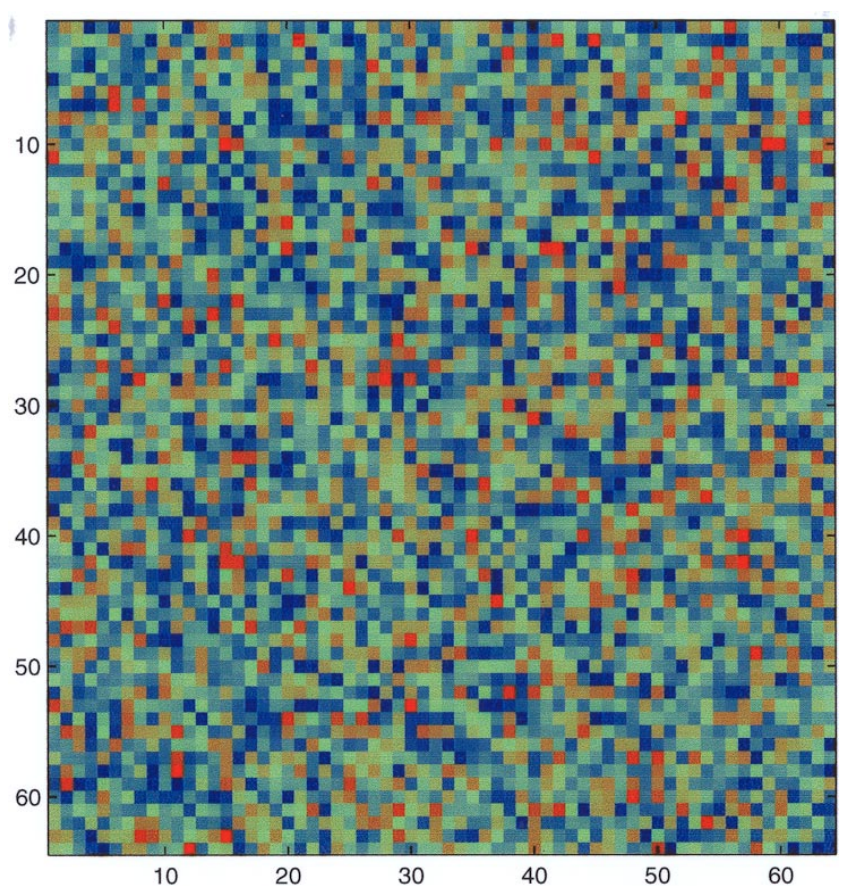

(b)

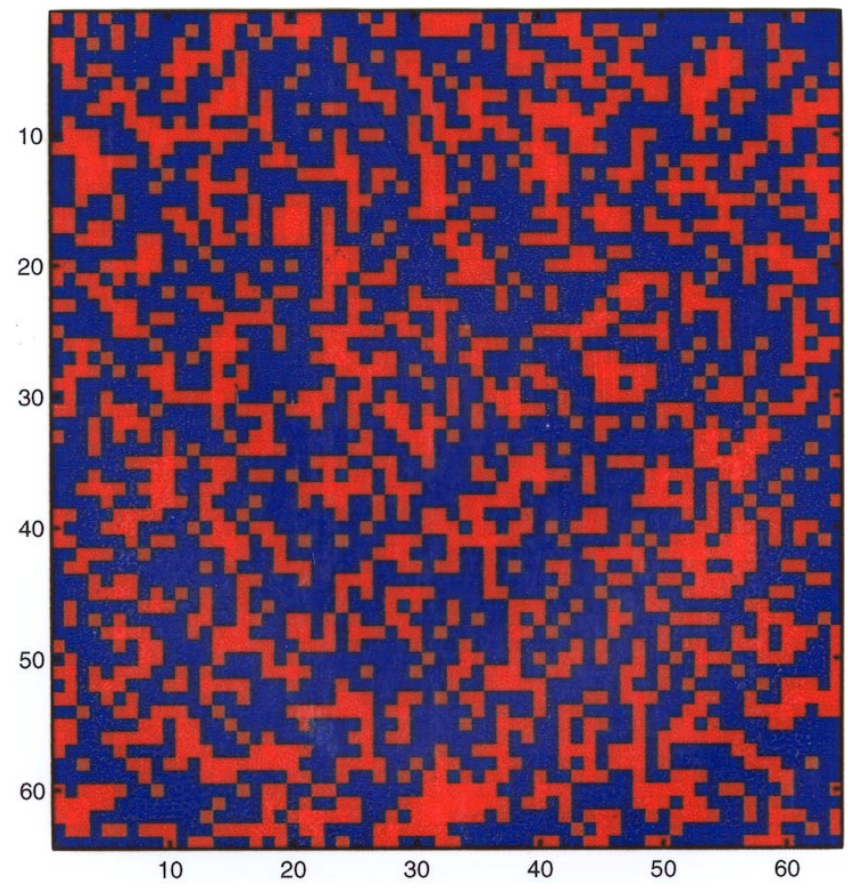

(c)

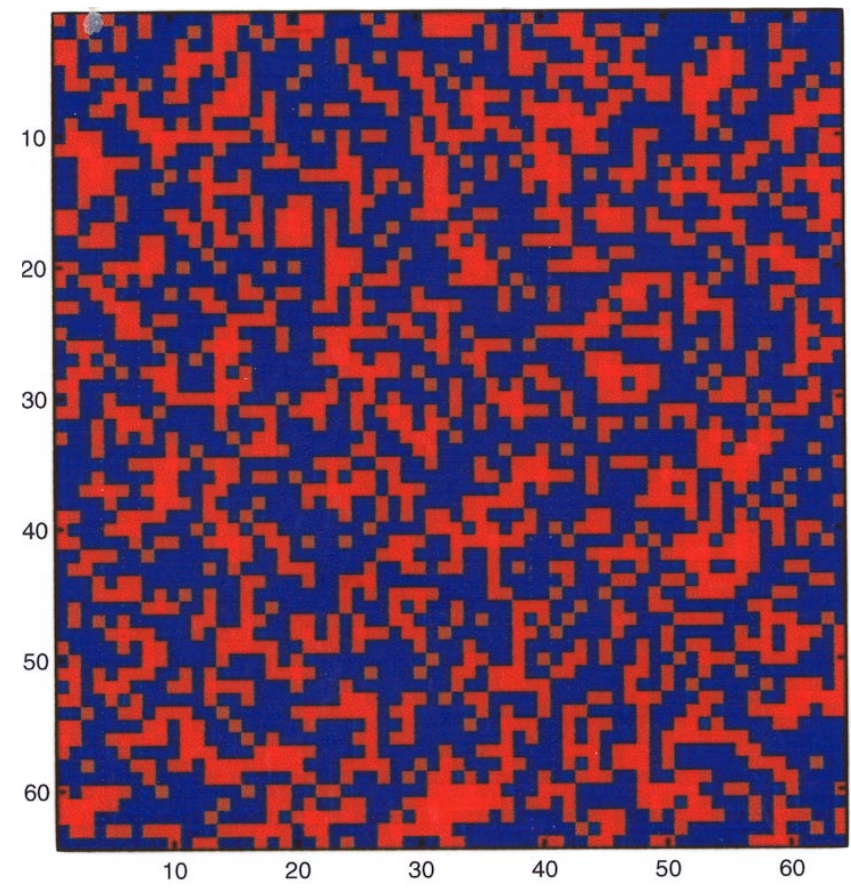

(d)

Fig. 14. Simulation results of a $64 \times 64 \mathrm{CNN}$ made of two-port second-order CNN cells where $\alpha=2, b=1, D_{1}=1$ and $D_{2}=1$ are fixed. This CNN is locally active. (a) $V_{1}(i, j)$ at $t=0.2$. (b) $V_{2}(i, j)$ at $t=0.2$. (c) $V_{1}(i, j)$ at $t=500$. (d) $V_{2}(i, j)$ at $t=500$. (e) $V_{1}(i, j)$ at $t=1000$. (f) $V_{2}(i, j)$ at $t=1000$. 


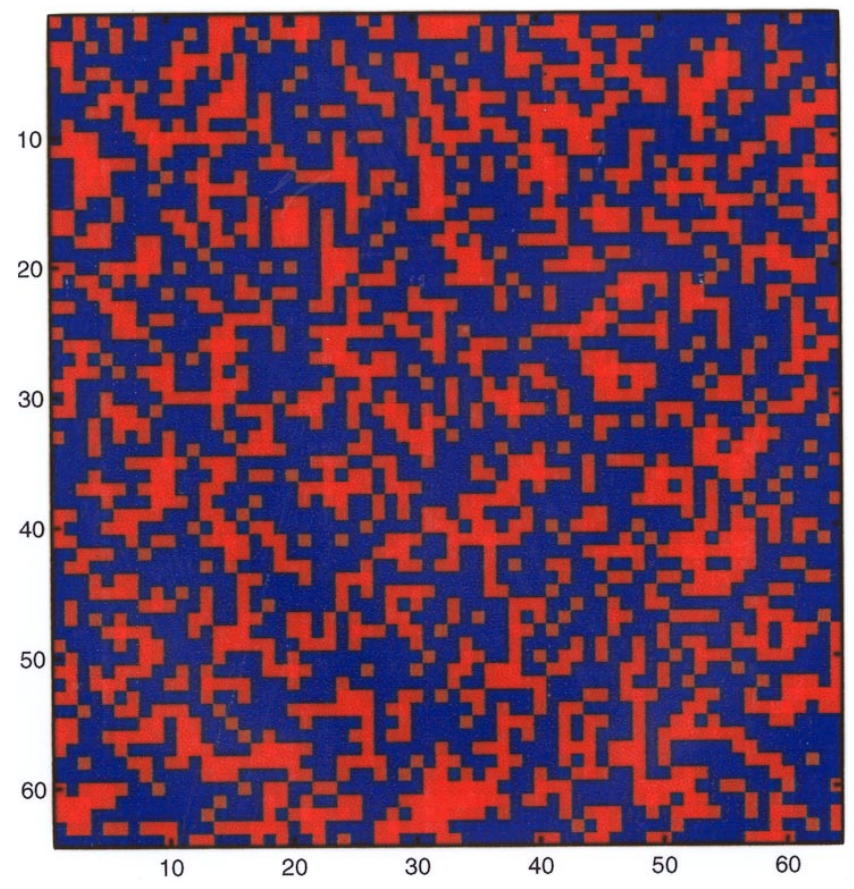

(e)

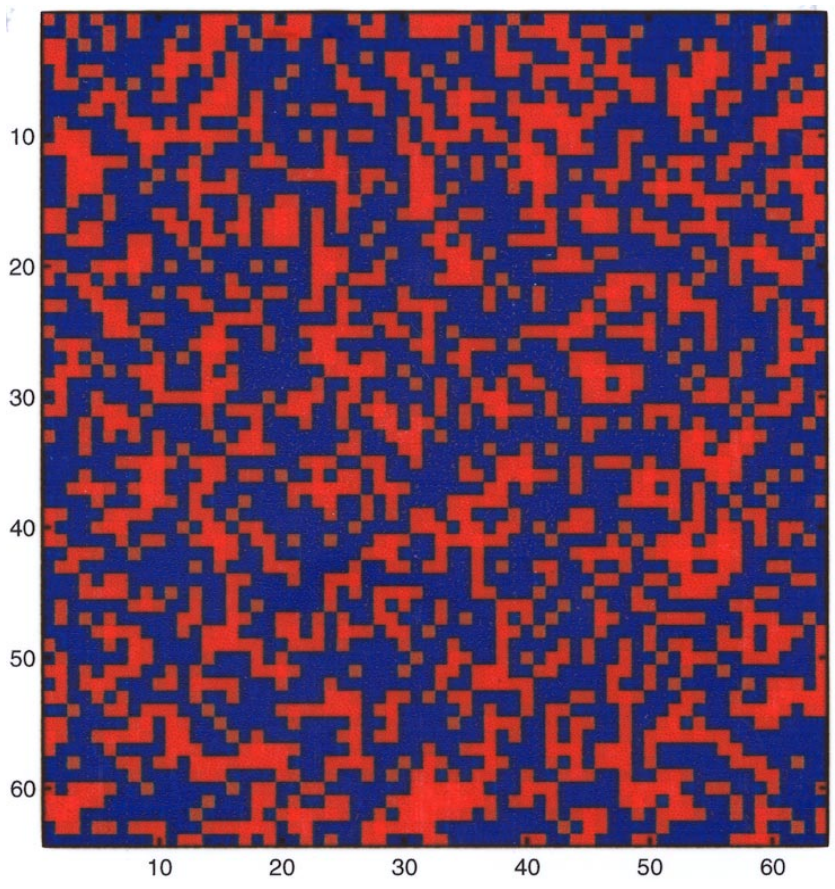

(f)

Fig. 14. (Continued)

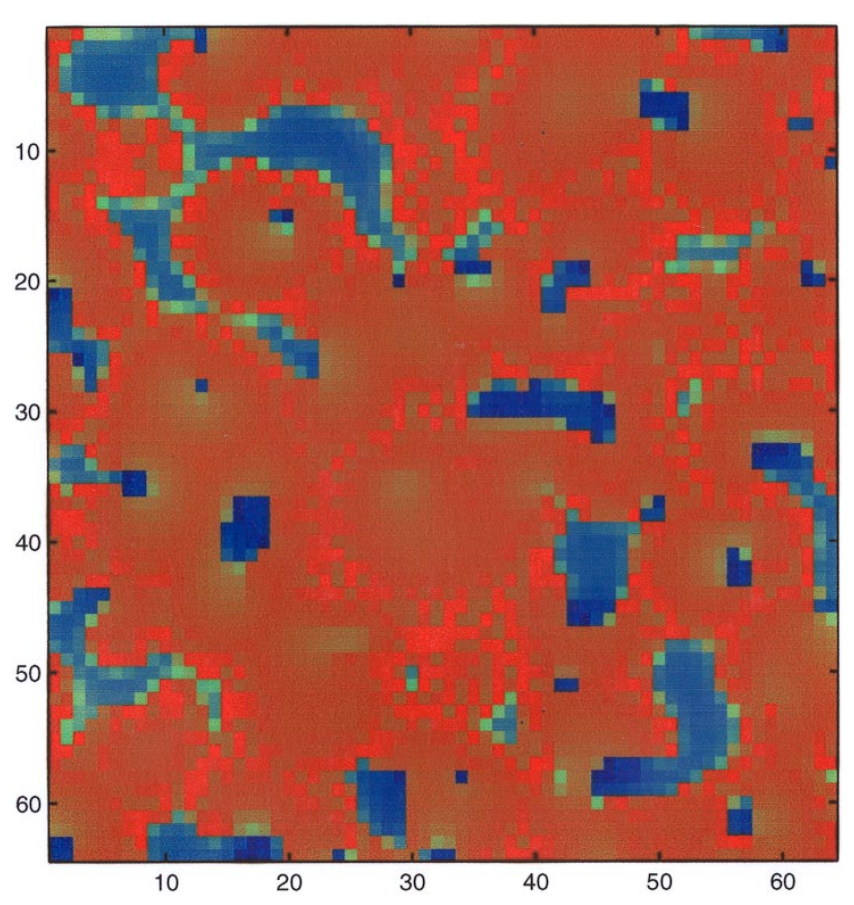

(a)

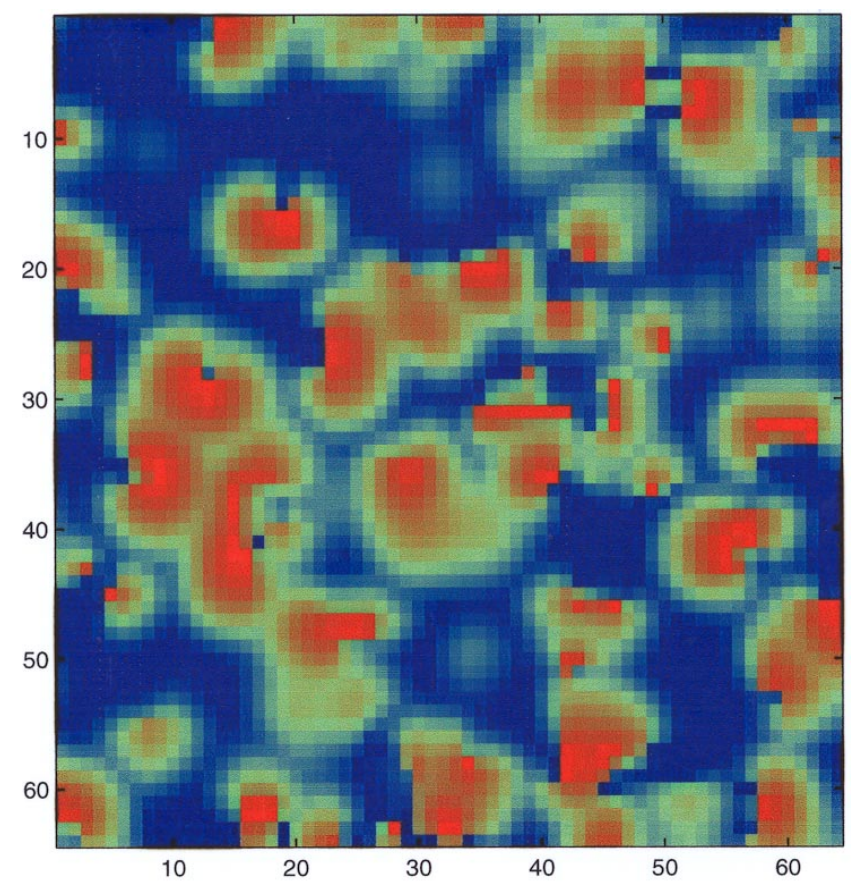

(b)

Fig. 15. Simulation results of a $64 \times 64 \mathrm{CNN}$ made of two-port second-order cells, where $\alpha=-1, b=10, D_{1}=1$ and $D_{2}=1$ are fixed. This CNN is locally active. (a) $V_{1}(i, j)$ at $t=20$. (b) $V_{2}(i, j)$ at $t=20$. (c) $V_{1}(i, j)$ at $t=40$. (d) $V_{2}(i, j)$ at $t=40$. (e) $V_{1}(i, j)$ at $t=45$. (f) $V_{2}(i, j)$ at $t=45$. (g) $V_{1}(i, j)$ at $t=90$. (h) $V_{2}(i, j)$ at $t=90$. (i) $V_{1}(i, j)$ at $t=100$. (j) $V_{2}(i, j)$ at $t=100$. (k) $V_{1}(i, j)$ at $t=200$. (l) $V_{2}(i, j)$ at $t=200$. (m) $V_{1}(i, j)$ at $t=300$. (n) $V_{2}(i, j)$ at $t=300$. (o) $V_{1}(i, j)$ at $t=400$. (p) $V_{2}(i, j)$ at $t=400$. (q) $V_{1}(i, j)$ at $t=600$. (r) $V_{2}(i, j)$ at $t=600$. (s) $V_{1}(i, j)$ at $t=800$. (t) $V_{2}(i, j)$ at $t=800$. (u) $V_{1}(i, j)$ at $t=1000$. (v) $V_{2}(i, j)$ at $t=1000$. (w) $V_{1}(i, j)$ at $t=2000$. (x) $V_{2}(i, j)$ at $t=2000$. 


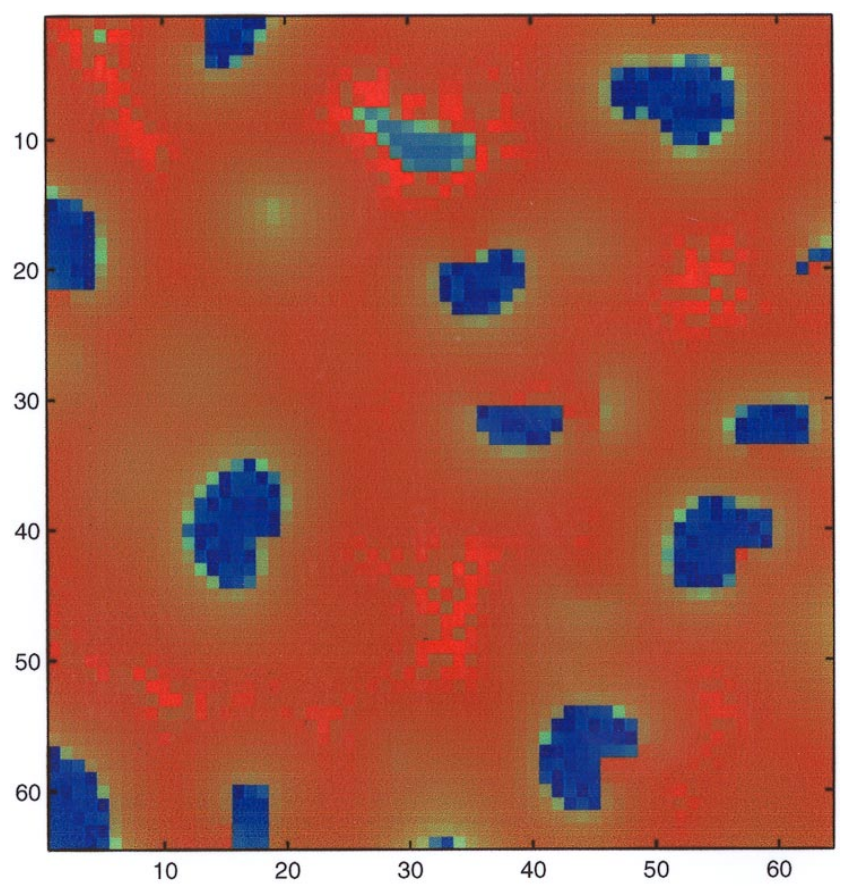

(c)

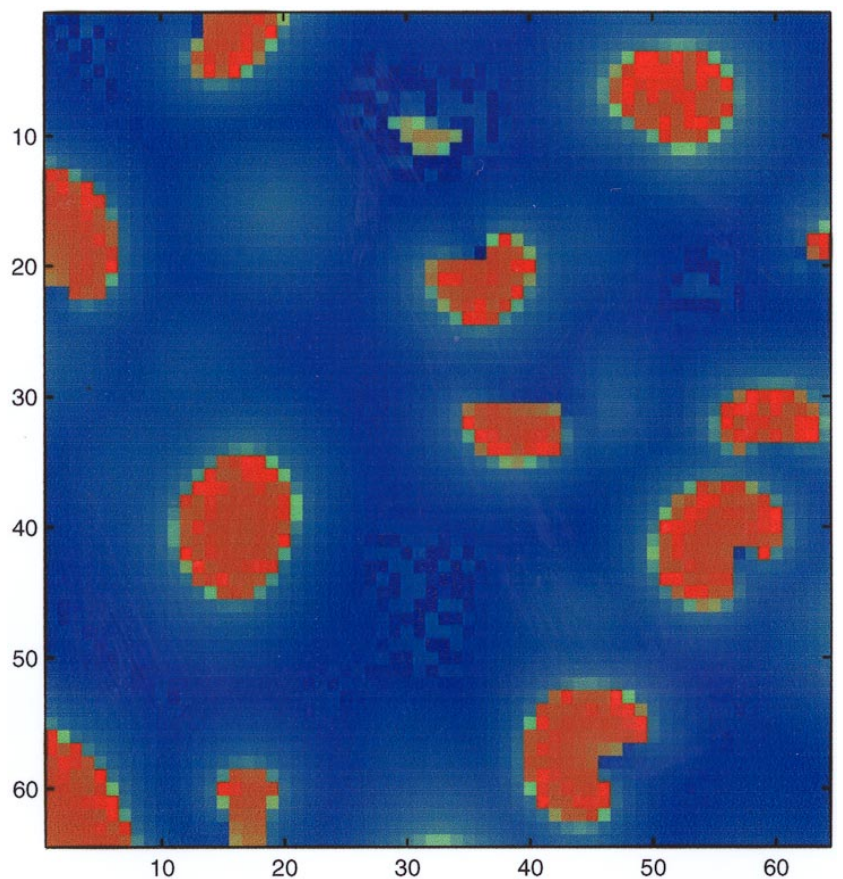

(e)

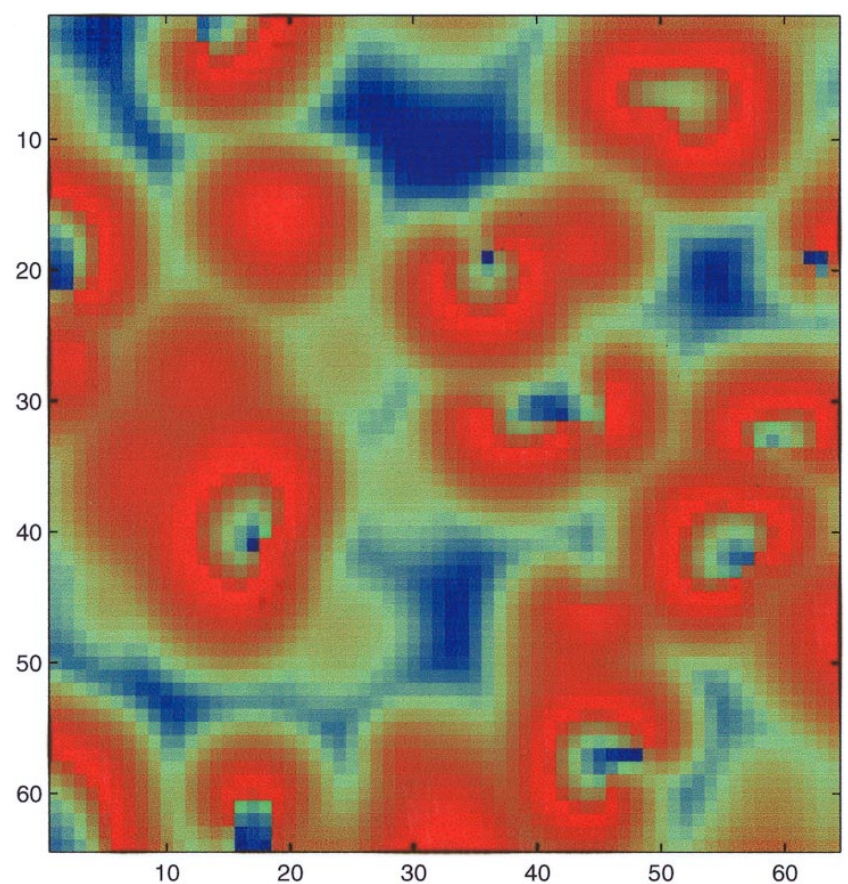

(d)

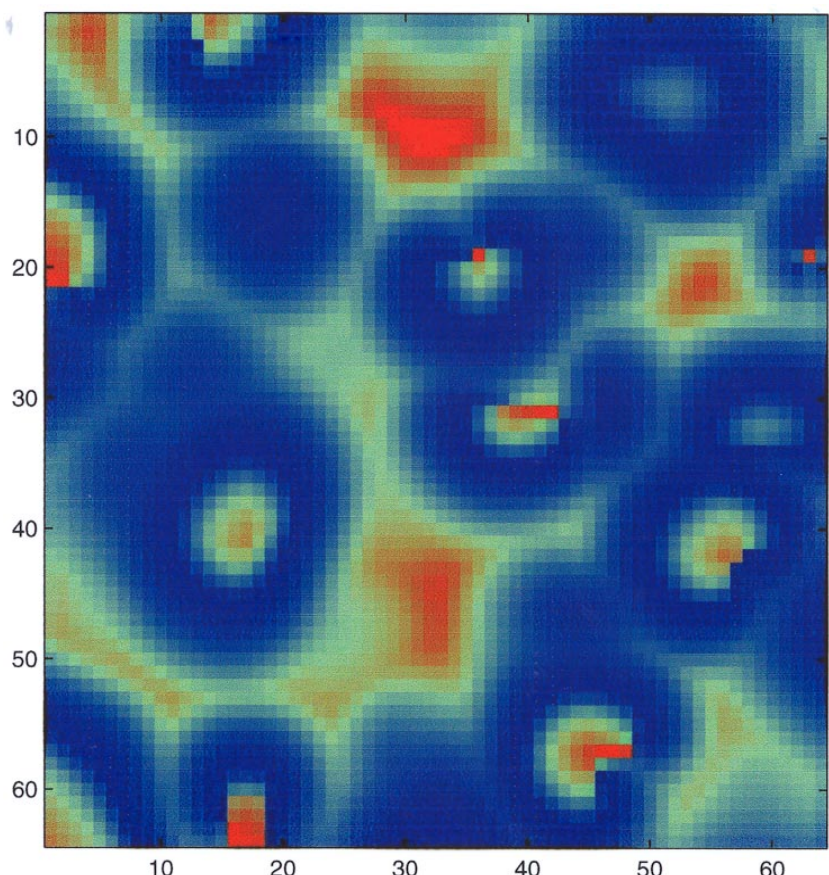

(f)

Fig. 15. (Continued) 


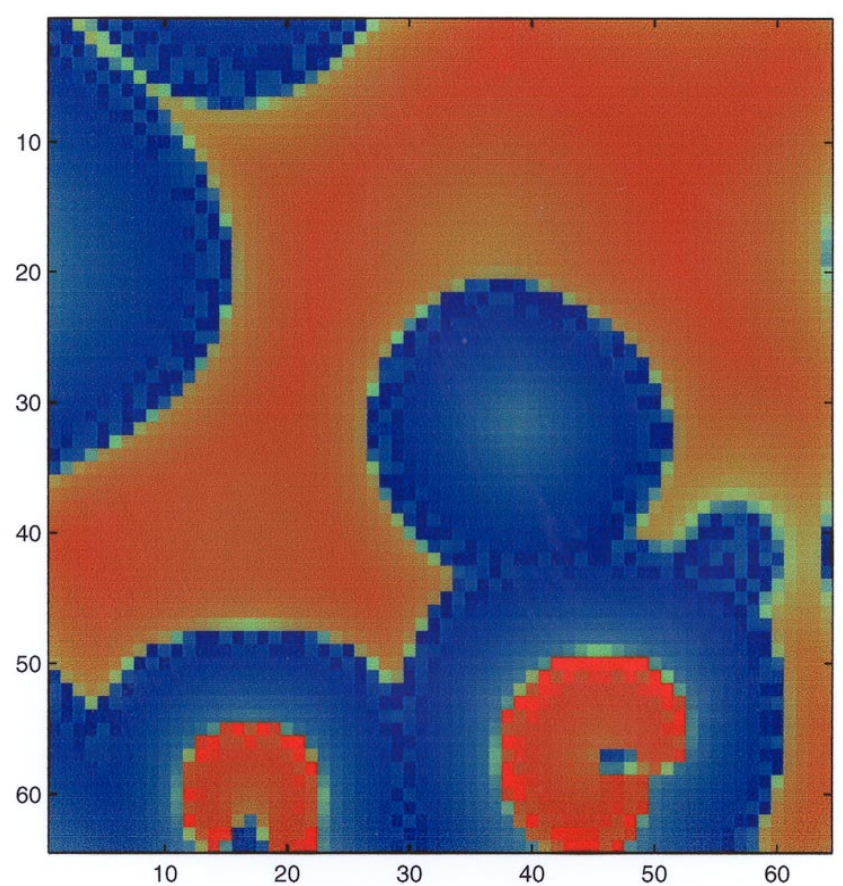

(g)

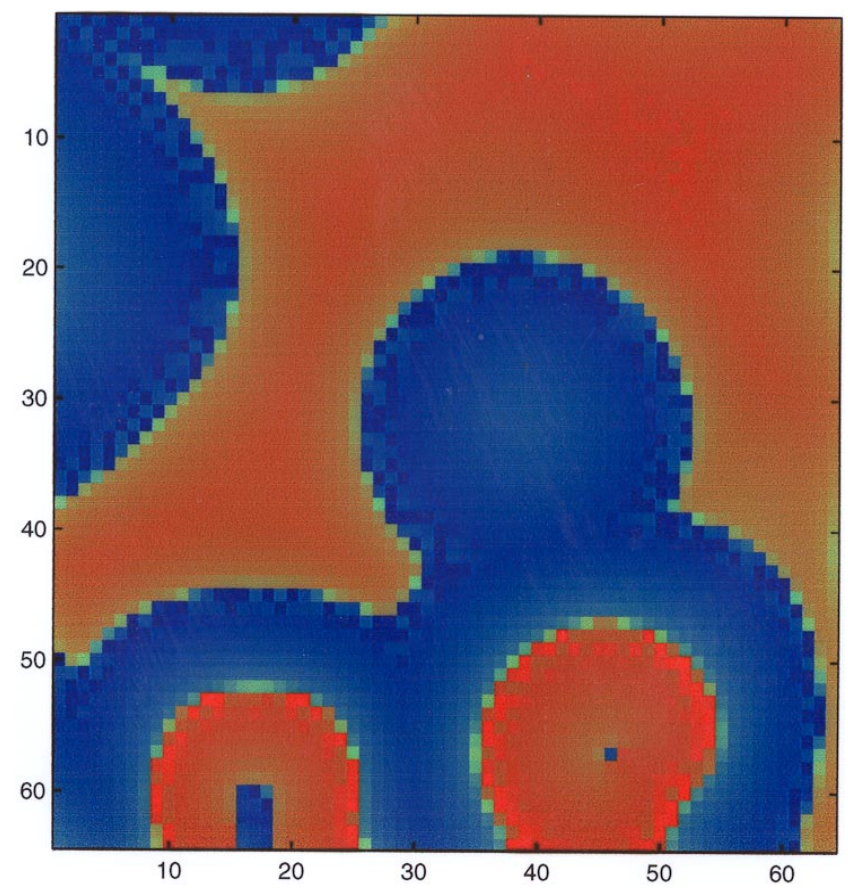

(i)

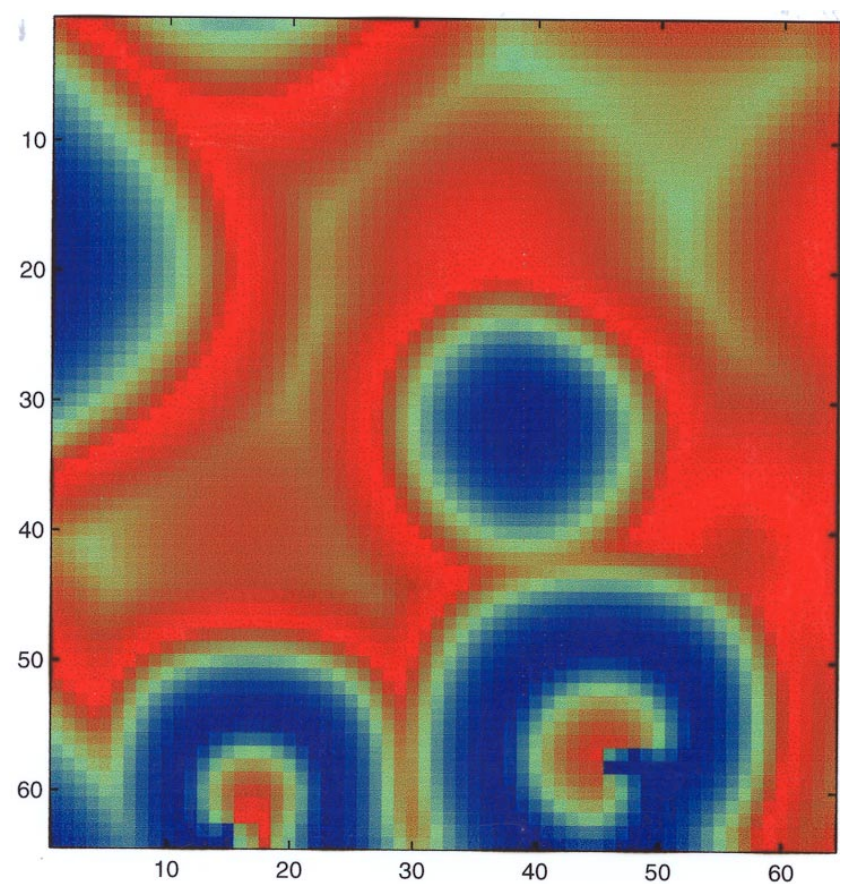

(h)

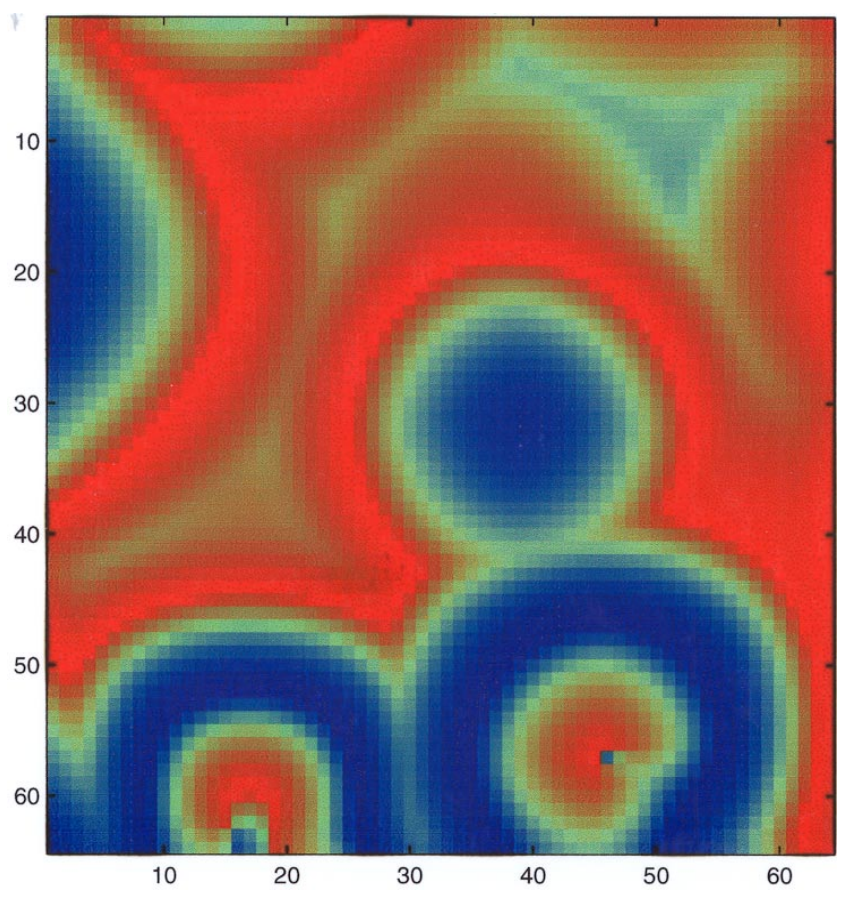

(j)

Fig. 15. (Continued) 


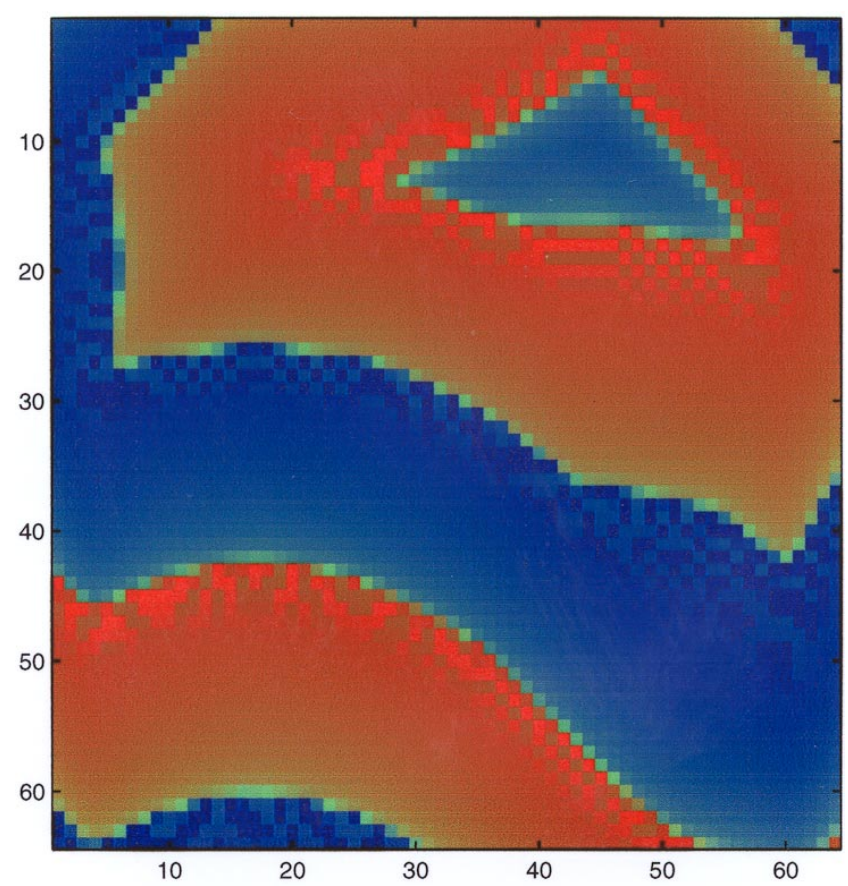

(k)

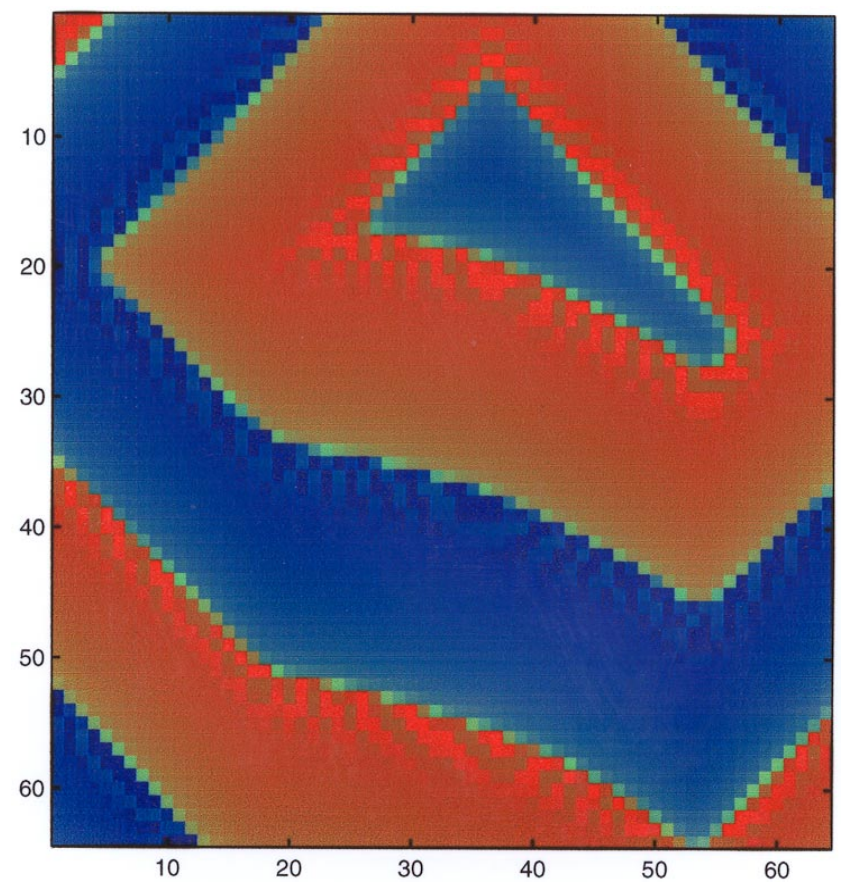

(m)

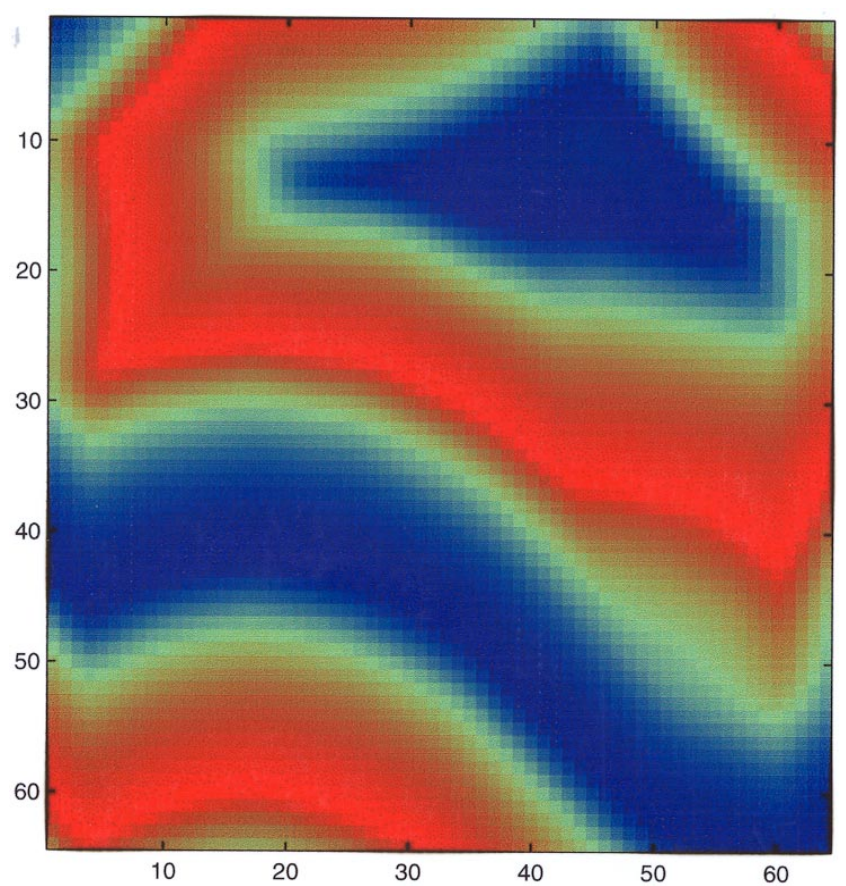

(l)

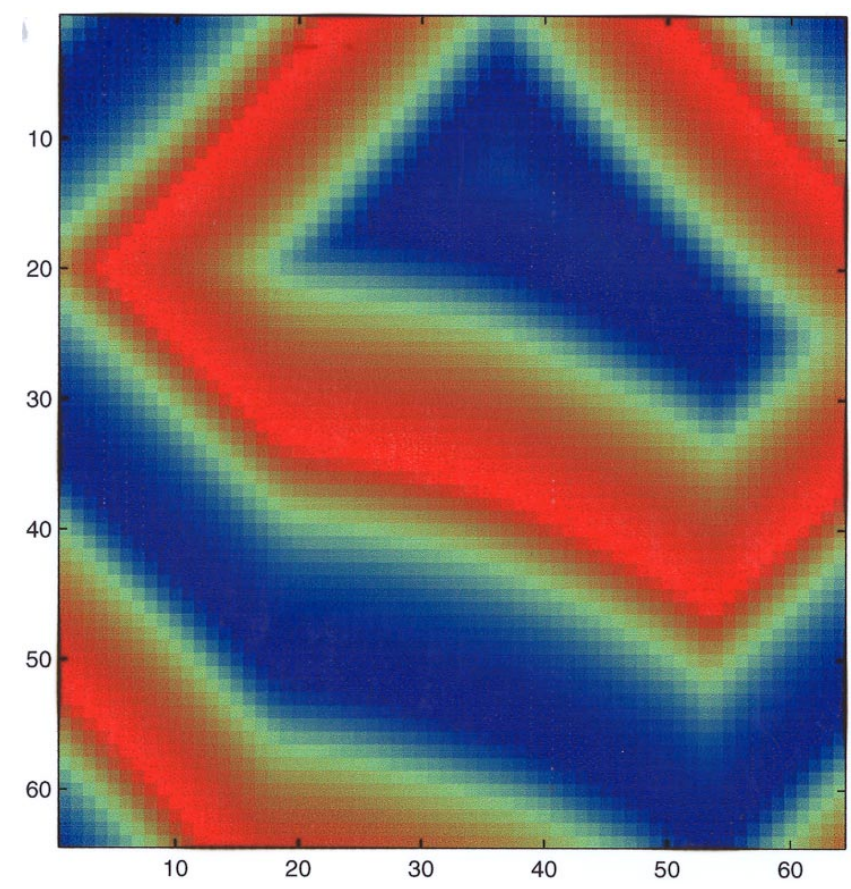

(n)

Fig. 15. (Continued) 


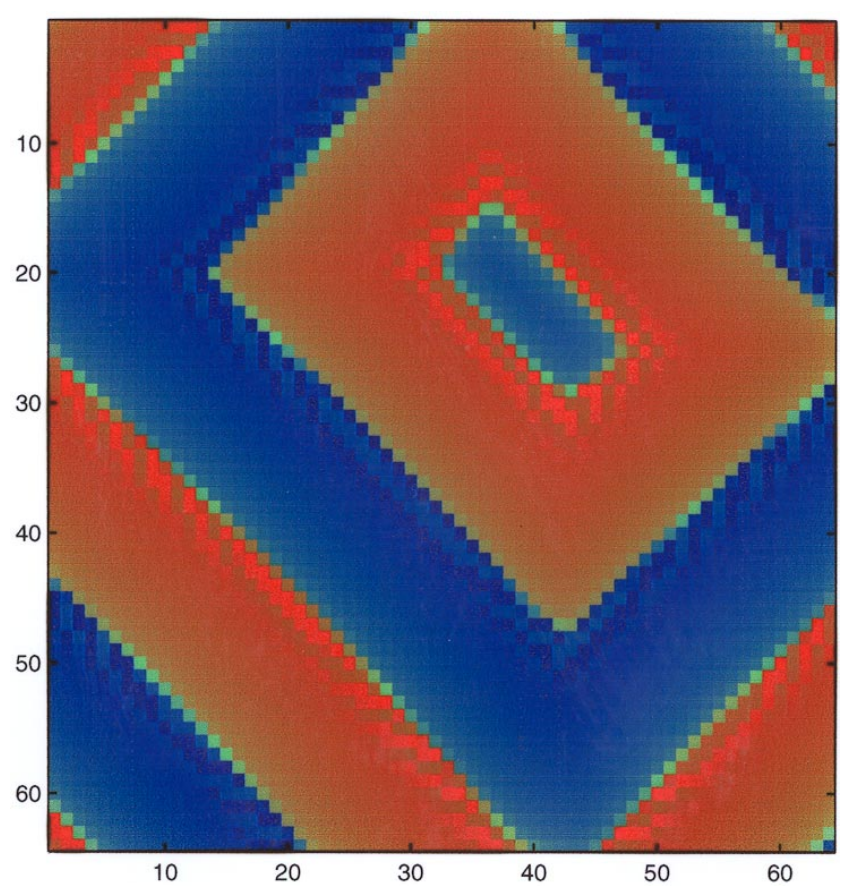

(o)

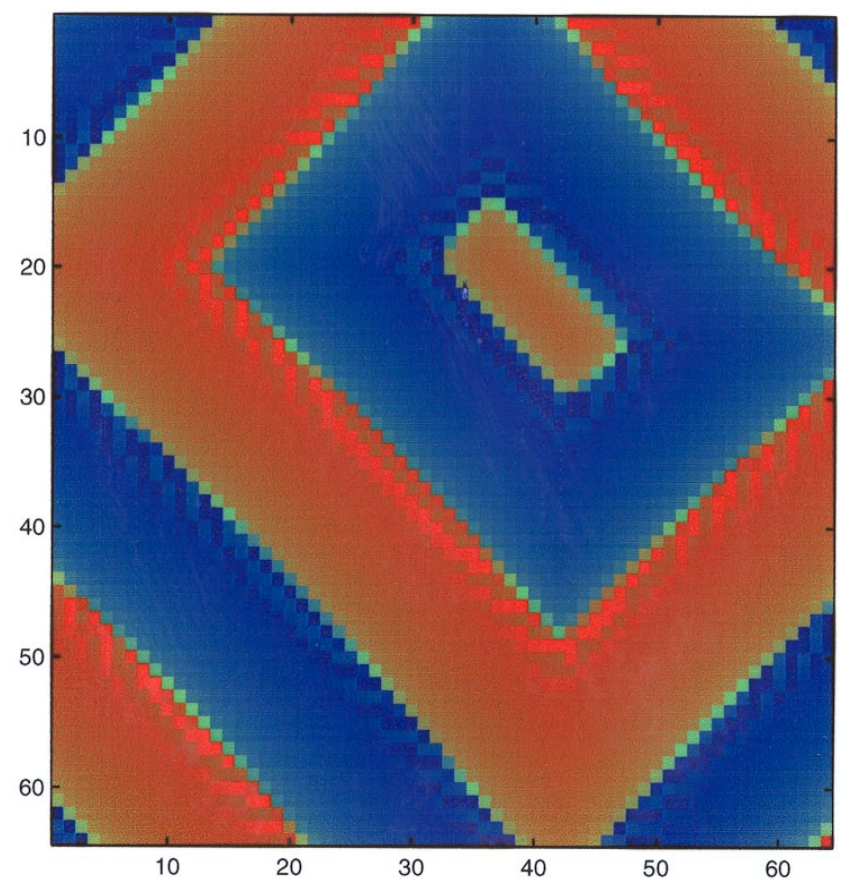

(q)

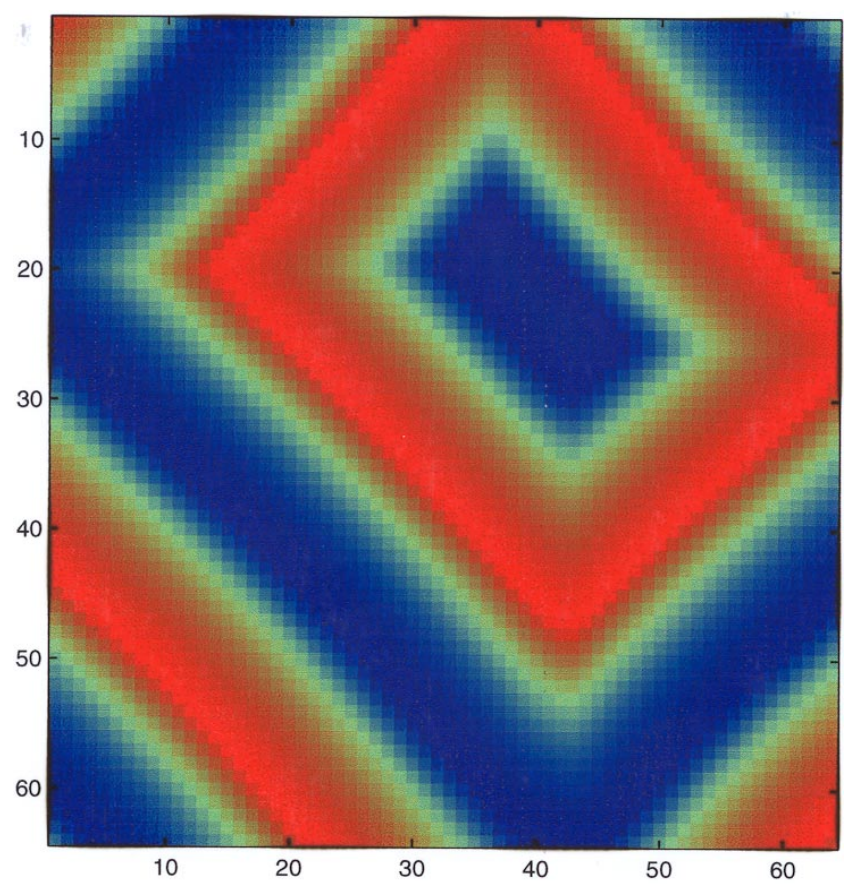

(p)

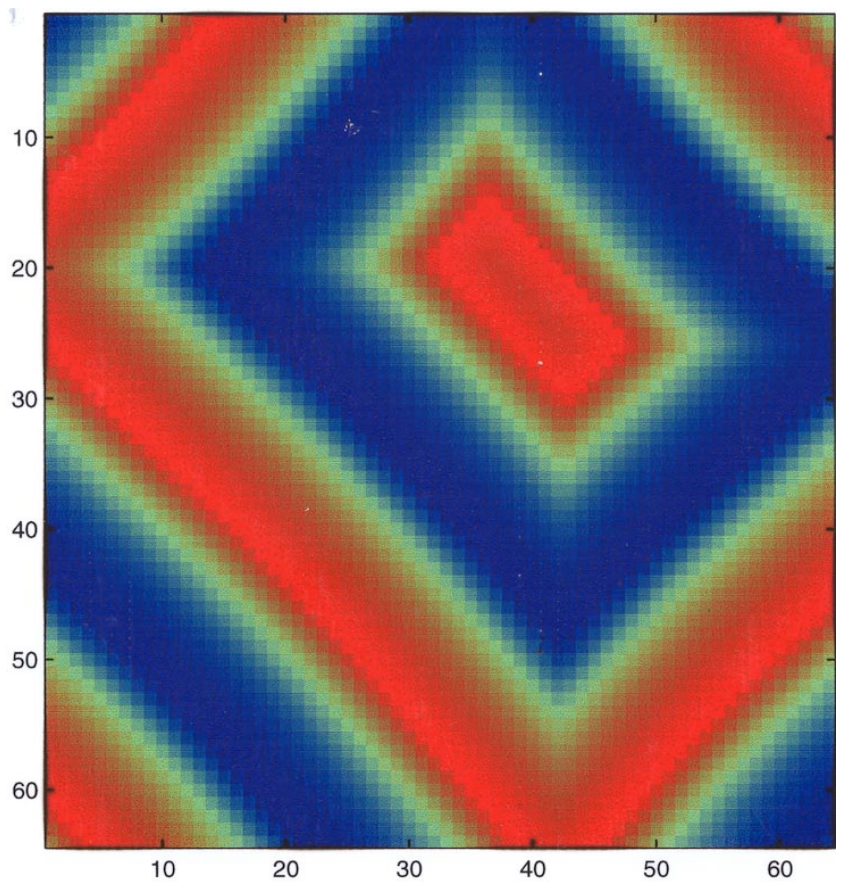

$(\mathrm{r})$

Fig. 15. (Continued) 


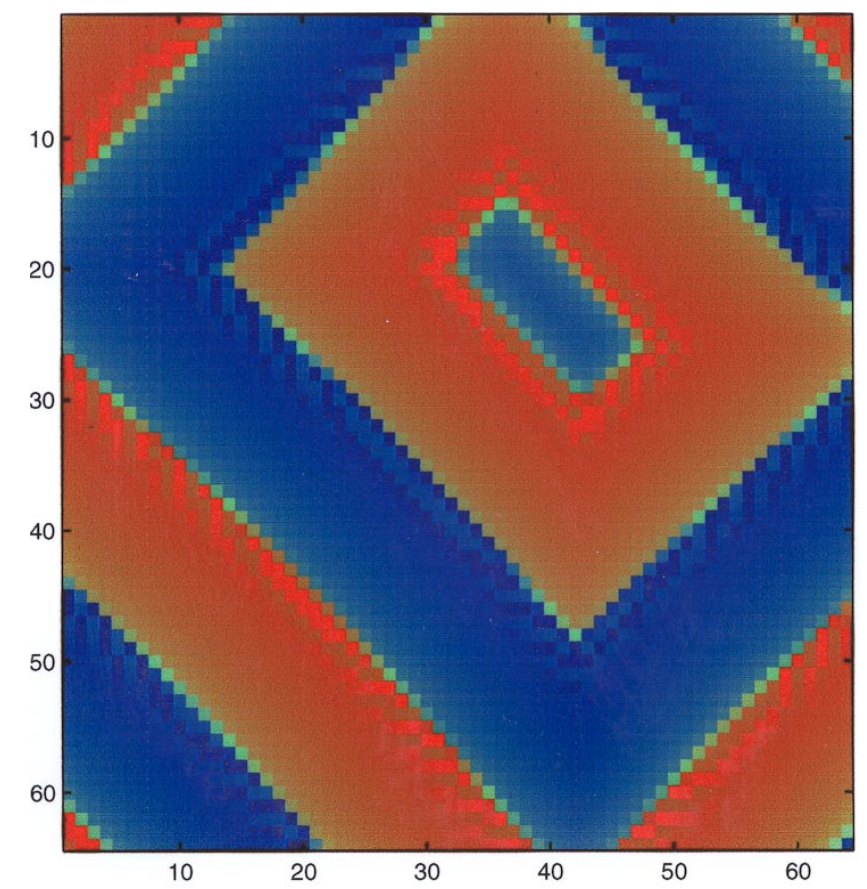

(s)

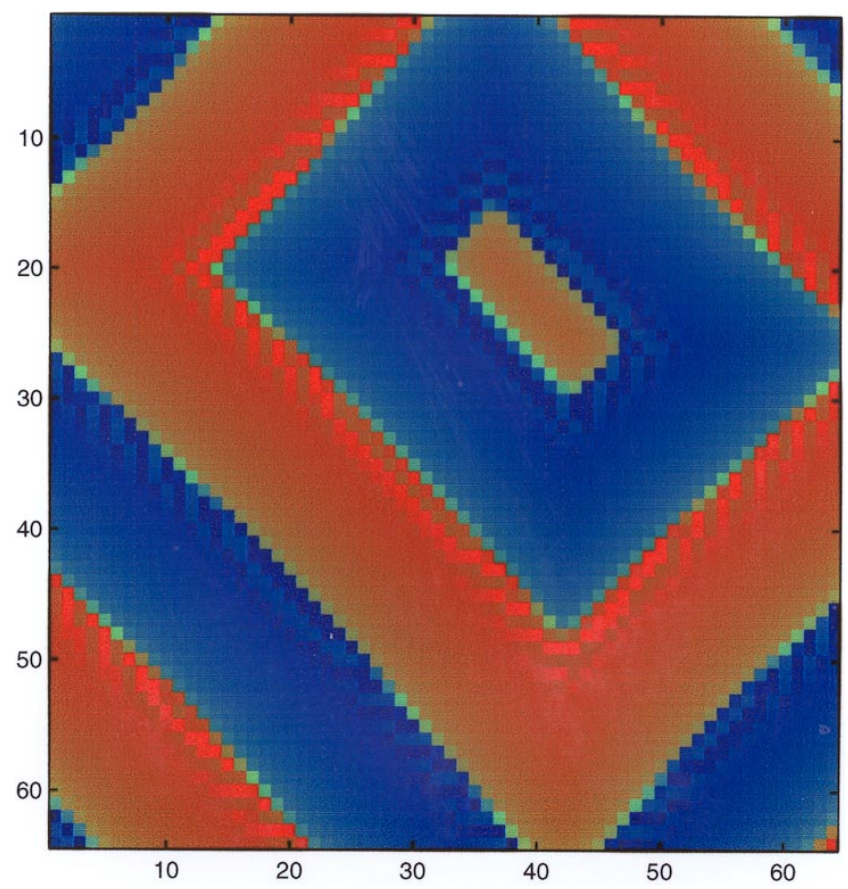

(u)

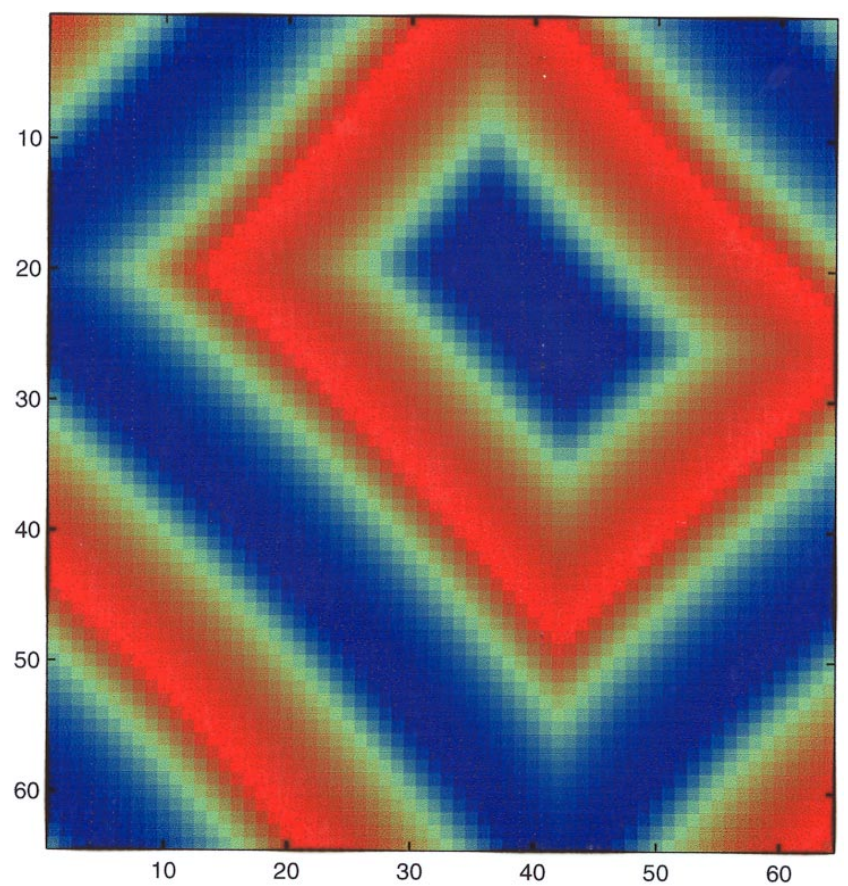

$(\mathrm{t})$

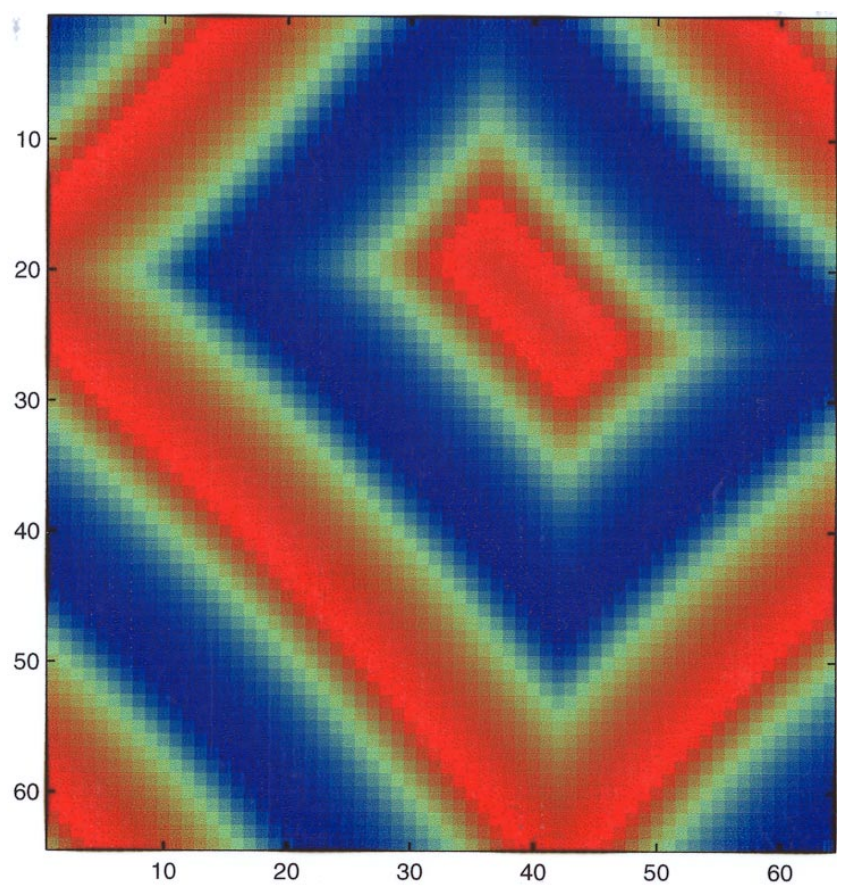

(v)

Fig. 15. (Continued) 


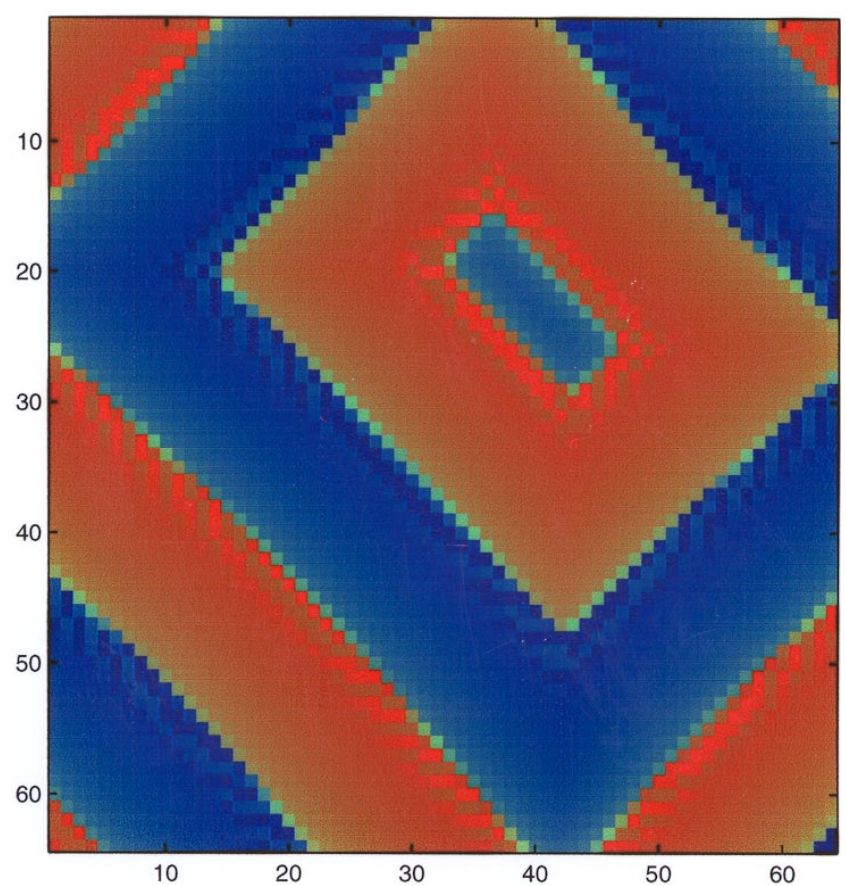

(w)

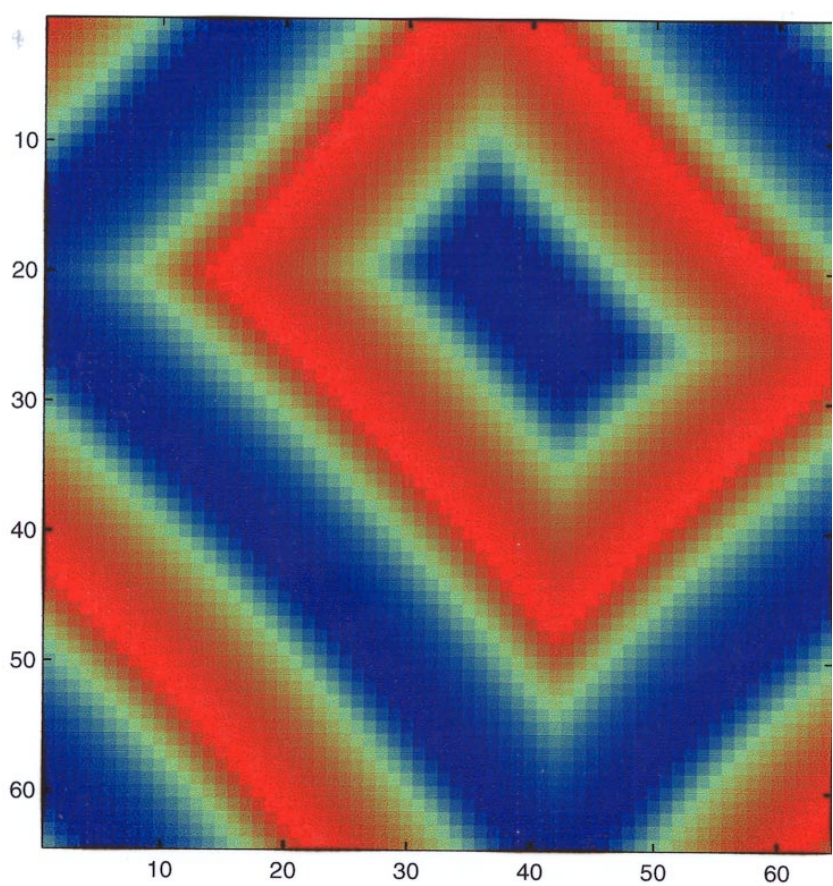

(x)

Fig. 15. (Continued)

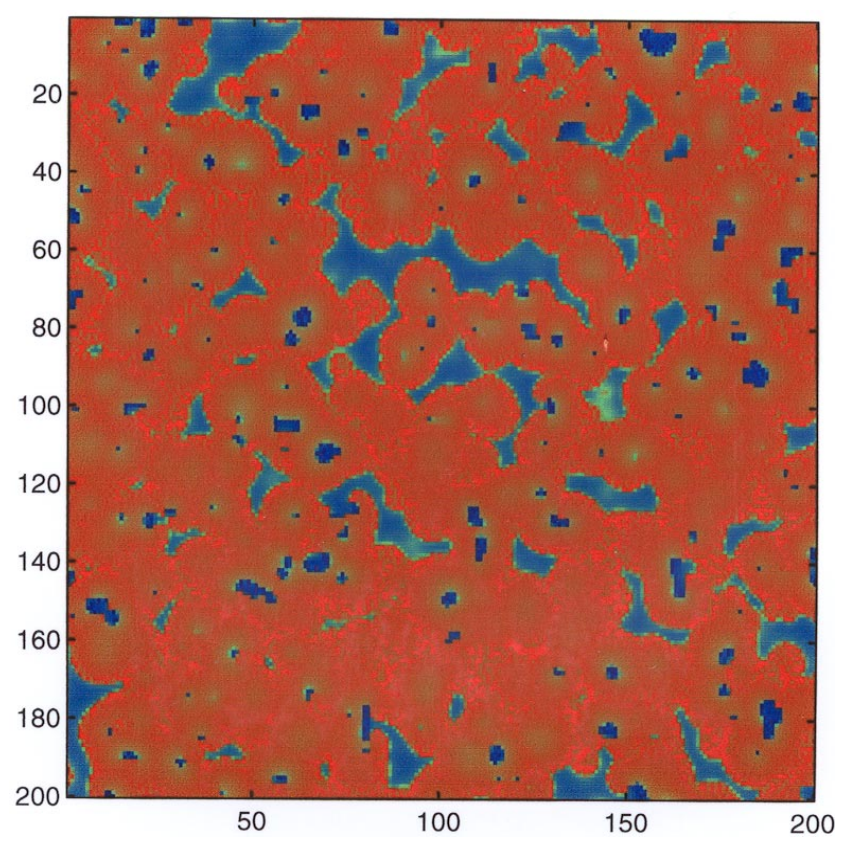

(a)

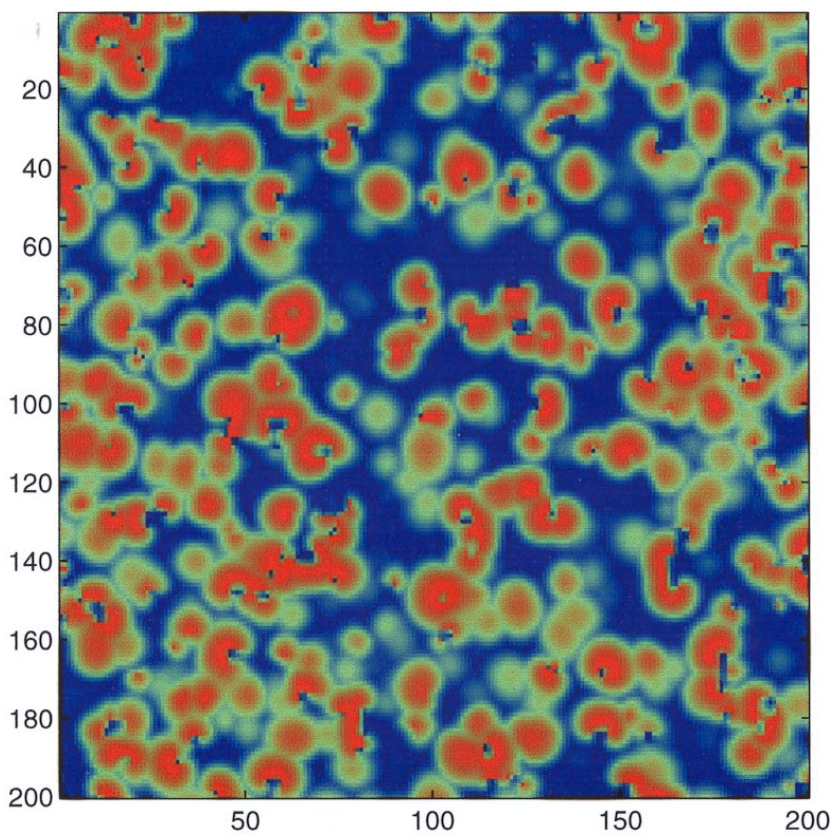

(b)

Fig. 16. The simulation results of a $200 \times 200 \mathrm{CNN}$ made of two-port second-order cells, where $\alpha=-1, b=10, D_{1}=1$ and $D_{2}=1$ are fixed. This CNN is locally active. (a) $V_{1}(i, j)$ at $t=30$. (b) $V_{2}(i, j)$ at $t=30$. (c) $V_{1}(i, j)$ at $t=60$. (d) $V_{2}(i, j)$ at $t=60$. (e) $V_{1}(i, j)$ at $t=1000$. (f) $V_{2}(i, j)$ at $t=1000$. (g) $V_{1}(i, j)$ at $t=2000$. (h) $V_{2}(i, j)$ at $t=2000$. 


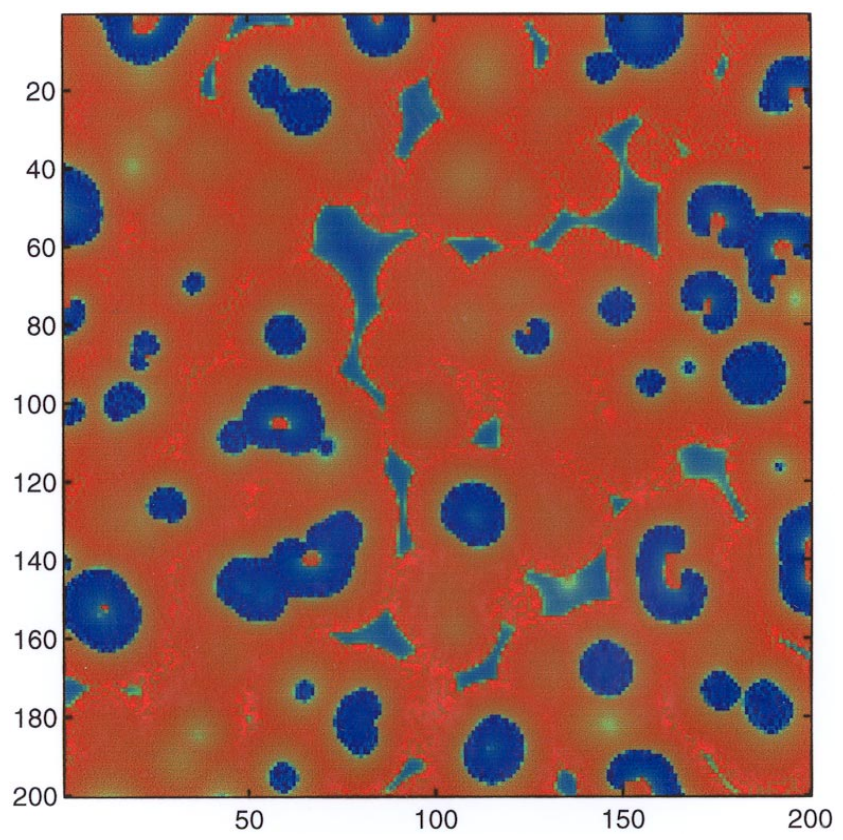

(c)

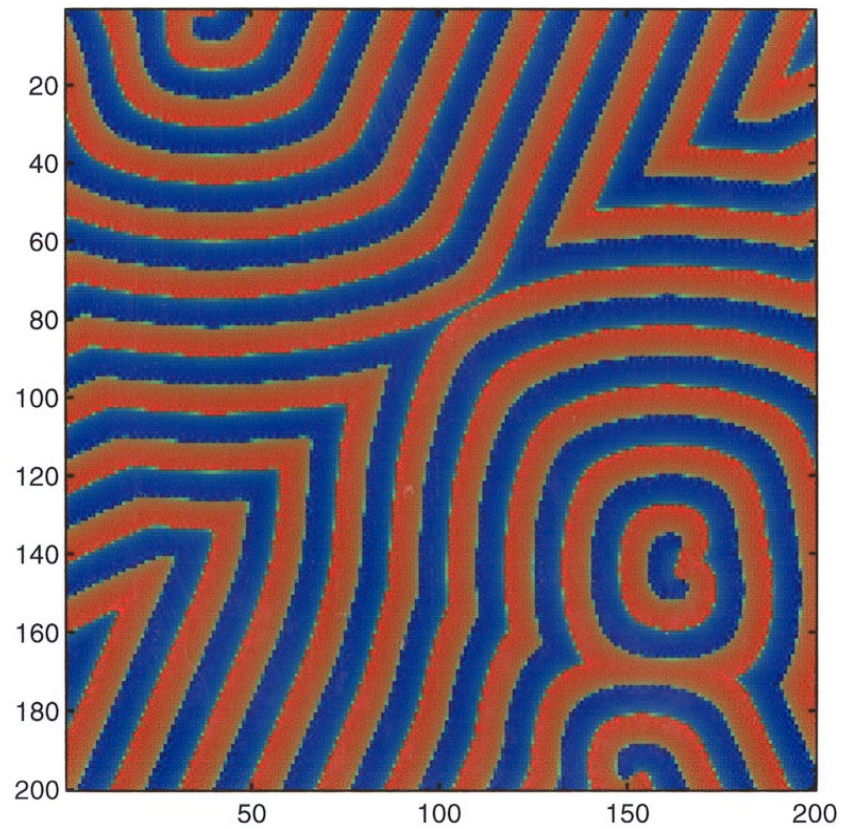

(e)

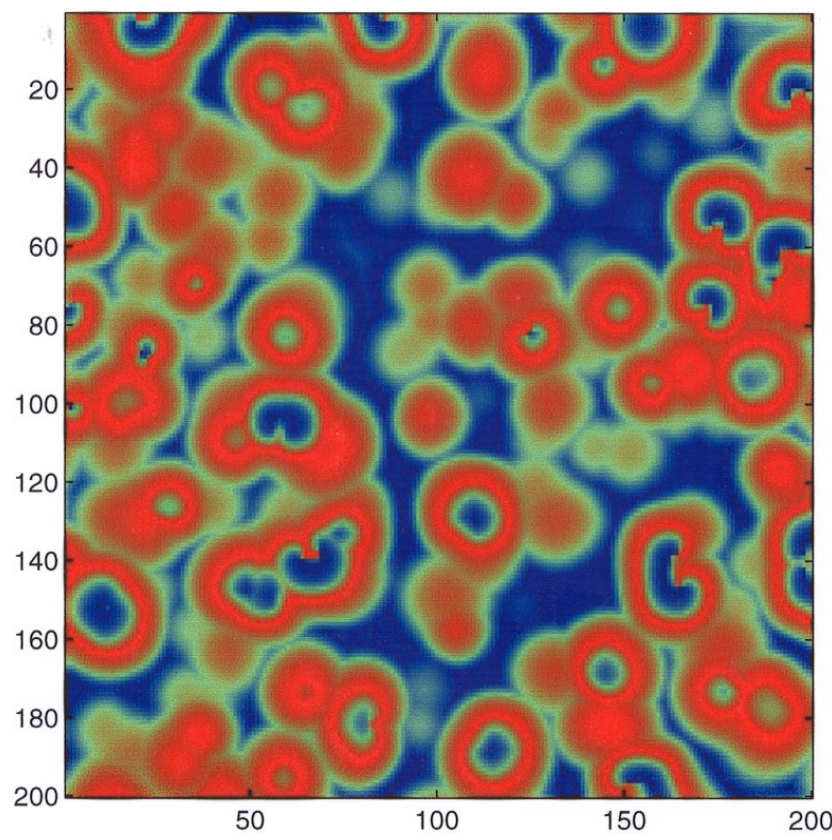

(d)

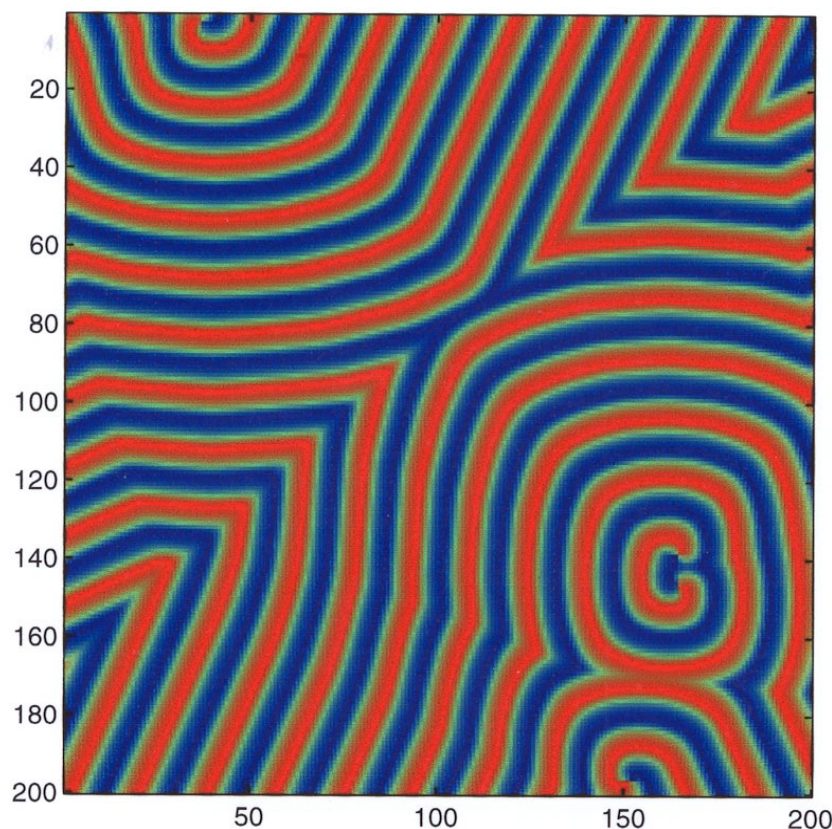

(f)

Fig. 16. (Continued) 


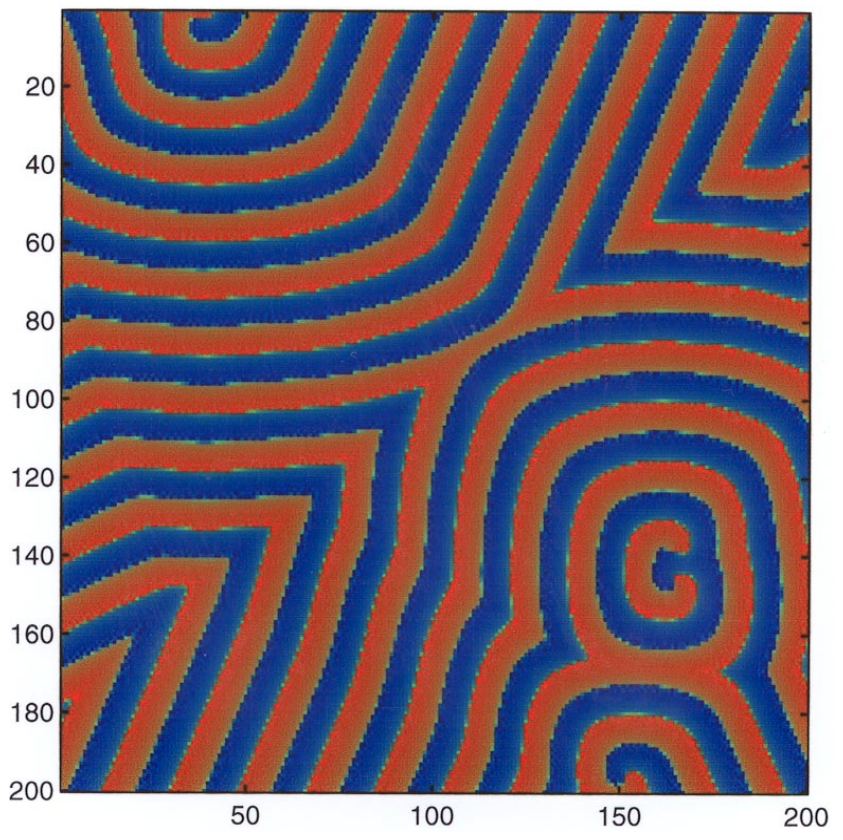

(g)

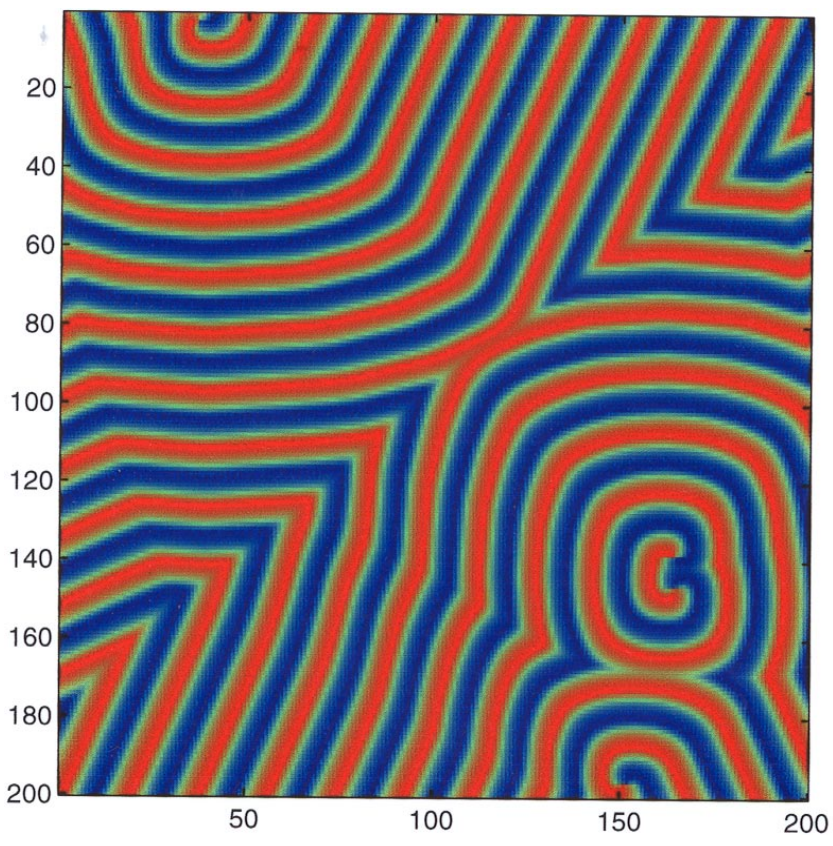

(h)

Fig. 16. (Continued)

1. Calculate $\mathbf{Z}_{Q}(s)$ and find the poles of $\mathbf{Z}_{Q}(s)$;

2. Calculate $\mathbf{Y}_{Q}(s)$ and find the local passive region by applying criteria $\mathrm{P} 1\left(\mathbf{Y}_{Q}(s)\right)$ to $\mathrm{P} 3\left(\mathbf{Y}_{Q}(s)\right)$. The local active region is then simply obtained by taking the set complement of the local passive region.

3. Find edge-of-chaos regions by using the following criterion:

[edge-of-chaos regions]

$=[$ local active region $]$

$\cap$ [parameter region where all poles of

$\mathbf{Z}_{Q}(s)$ are in the left-half $s$-plane]

The linearized state equation of a two-port third-order CNN cell at an equilibrium point $Q$ is given by

$$
\begin{aligned}
& \dot{v}_{1}=a_{11} v_{1}+a_{12} v_{2}+a_{13} v_{3}+i_{1}, \\
& \dot{v}_{2}=a_{21} v_{1}+a_{22} v_{2}+a_{23} v_{3}+i_{2}, \\
& \dot{v}_{3}=a_{31} v_{1}+a_{32} v_{2}+a_{33} v_{3} .
\end{aligned}
$$

\subsection{Poles of $\mathrm{Z}_{Q}(s)$}

Let us find the poles of $\mathbf{Z}_{Q}(s)$. Let $\hat{v}_{1}, \hat{v}_{2}$ and $\hat{v}_{3}$ be the Laplace transforms of $v_{1}(t), v_{2}(t)$ and $v_{3}(t)$, respectively. Applying Laplace transform to the third equation in Eq. (170) and solving for $\hat{v}_{3}$ in terms of $\hat{v}_{1}$ and $\hat{v}_{2}$, we obtain

$$
\hat{v}_{3}=\frac{1}{s-a_{33}}\left(a_{31} \hat{v}_{1}+a_{32} \hat{v}_{2}\right) .
$$

Substituting Eq. (171) for $\hat{v}_{3}$ in the Laplace transform of the first two equations in Eq. (170), we obtain

$$
\begin{aligned}
s \hat{v}_{1}= & a_{11} \hat{v}_{1}+a_{12} \hat{v}_{2} \\
& +\frac{a_{13}}{s-a_{33}}\left(a_{31} \hat{v}_{1}+a_{32} \hat{v}_{2}\right)+\hat{i}_{1}, \\
s \hat{v}_{2}= & a_{21} \hat{v}_{1}+a_{22} \hat{v}_{2} \\
& +\frac{a_{23}}{s-a_{33}}\left(a_{31} \hat{v}_{1}+a_{32} \hat{v}_{2}\right)+\hat{i}_{2} .
\end{aligned}
$$

Solving for $\hat{v}_{1}$ and $\hat{v}_{2}$ in terms of $\hat{i}_{1}$ and $\hat{i}_{2}$, we obtain the $2 \times 2$ cell impedance matrix 


$$
\begin{aligned}
& \mathbf{Z}_{Q}(s)=\left[\begin{array}{cc}
s-a_{11}-\frac{a_{13} a_{31}}{s-a_{33}} & -a_{12}-\frac{a_{13} a_{32}}{s-a_{33}} \\
-a_{21}-\frac{a_{23} a_{31}}{s-a_{33}} & s-a_{22}-\frac{a_{23} a_{32}}{s-a_{33}}
\end{array}\right]^{-1} \\
& =\left(\frac{1}{\left(s-a_{11}-\frac{a_{13} a_{31}}{s-a_{33}}\right)\left(s-a_{22}-\frac{a_{23} a_{32}}{s-a_{33}}\right)-\left(a_{12}+\frac{a_{13} a_{32}}{s-a_{33}}\right)\left(a_{21}+\frac{a_{23} a_{31}}{s-a_{33}}\right)}\right) \\
& \times\left[\begin{array}{cc}
s-a_{22}-\frac{a_{23} a_{32}}{s-a_{33}} & a_{12}+\frac{a_{13} a_{32}}{s-a_{33}} \\
a_{21}+\frac{a_{23} a_{31}}{s-a_{33}} & s-a_{11}-\frac{a_{13} a_{31}}{s-a_{33}}
\end{array}\right] \\
& =\left(s-a_{33}\right)\left\{\left[s^{2}-\left(a_{11}+a_{33}\right) s+a_{11} a_{33}-a_{13} a_{31}\right]\left[s^{2}-\left(a_{22}+a_{33}\right) s+a_{22} a_{33}-a_{23} a_{32}\right]\right. \\
& \left.-\left(a_{12} s-a_{12} a_{33}+a_{13} a_{32}\right)\left(a_{21} s-a_{21} a_{33}+a_{23} a_{31}\right)\right\}^{-1} \\
& \times\left[\begin{array}{cc}
s^{2}-\left(a_{22}+a_{33}\right) s+a_{22} a_{33}-a_{23} a_{32} & a_{12} s-a_{12} a_{33}+a_{13} a_{32} \\
a_{21} s-a_{21} a_{33}+a_{23} a_{31} & s^{2}-\left(a_{11}+a_{33}\right) s+a_{11} a_{33}-a_{13} a_{31}
\end{array}\right] \\
& =\left(s-a_{33}\right)\left\{s^{4}-\left[a_{11}+a_{22}+2 a_{33}\right] s^{3}+\left(a_{11} a_{33}-a_{13} a_{31}+a_{22} a_{33}-a_{23} a_{32}\right.\right. \\
& \left.+\left(a_{11}+a_{33}\right)\left(a_{22}+a_{33}\right)-a_{12} a_{21}\right) s^{2}-\left(a_{11}+a_{33}\right)\left(a_{22} a_{33}-a_{23} a_{32}\right) \\
& \left.+\left(a_{22}+a_{33}\right)\left(a_{11} a_{33}-a_{13} a_{31}\right)+a_{12}\left(-a_{21} a_{33}+a_{23} a_{31}\right)+a_{21}\left(-a_{12} a_{33}+a_{13} a_{32}\right)\right) s \\
& \left.+\left(\left(a_{11} a_{33}-a_{13} a_{31}\right)\left(a_{22} a_{33}-a_{23} a_{32}\right)-\left(-a_{12} a_{33}+a_{13} a_{32}\right)\left(-a_{21} a_{33}+a_{23} a_{31}\right)\right) s^{0}\right\}^{-1} \\
& \times\left[\begin{array}{cc}
s^{2}-\left(a_{22}+a_{33}\right) s+a_{22} a_{33}-a_{23} a_{32} & a_{12} s-a_{12} a_{33}+a_{13} a_{32} \\
a_{21} s-a_{21} a_{33}+a_{23} a_{31} & s^{2}-\left(a_{11}+a_{33}\right) s+a_{11} a_{33}-a_{13} a_{31}
\end{array}\right] .
\end{aligned}
$$

Therefore, the poles of $\mathbf{Z}_{Q}(s)$ are the roots of the following polynomial:

$$
\begin{aligned}
P(s) \triangleq & s^{4}-\left[a_{11}+a_{22}+2 a_{33}\right] s^{3}+\left[a_{11} a_{33}-a_{13} a_{31}+a_{22} a_{33}-a_{23} a_{32}+\left(a_{11}+a_{33}\right)\left(a_{22}+a_{33}\right)-a_{12} a_{21}\right] s^{2} \\
& -\left[\left(a_{11}+a_{33}\right)\left(a_{22} a_{33}-a_{23} a_{32}\right)+\left(a_{22}+a_{33}\right)\left(a_{11} a_{33}-a_{13} a_{31}\right)+a_{12}\left(-a_{21} a_{33}+a_{23} a_{31}\right)\right. \\
& \left.+a_{21}\left(-a_{12} a_{33}+a_{13} a_{32}\right)\right] s+\left[\left(a_{11} a_{33}-a_{13} a_{31}\right)\left(a_{22} a_{33}-a_{23} a_{32}\right)\right. \\
& \left.-\left(-a_{12} a_{33}+a_{13} a_{32}\right)\left(-a_{21} a_{33}+a_{23} a_{31}\right)\right] \\
= & s^{4}+p_{3} s^{3}+p_{2} s^{2}+p_{1} s+p_{0}
\end{aligned}
$$

where

$$
\begin{aligned}
p_{3} \triangleq & -\left[a_{11}+a_{22}+2 a_{33}\right] \\
p_{2} \triangleq & a_{11} a_{33}-a_{13} a_{31}+a_{22} a_{33}-a_{23} a_{32}+\left(a_{11}+a_{33}\right)\left(a_{22}+a_{33}\right)-a_{12} a_{21} \\
p_{1} \triangleq & -\left[\left(a_{11}+a_{33}\right)\left(a_{22} a_{33}-a_{23} a_{32}\right)+\left(a_{22}+a_{33}\right)\left(a_{11} a_{33}-a_{13} a_{31}\right)+a_{12}\left(-a_{21} a_{33}+a_{23} a_{31}\right)\right. \\
& \left.\quad+a_{21}\left(-a_{12} a_{33}+a_{13} a_{32}\right)\right] \\
p_{0} \triangleq & \left(a_{11} a_{33}-a_{13} a_{31}\right)\left(a_{22} a_{33}-a_{23} a_{32}\right)-\left(-a_{12} a_{33}+a_{13} a_{32}\right)\left(-a_{21} a_{33}+a_{23} a_{31}\right) .
\end{aligned}
$$


Let us rewrite Eq. (173) into the more compact form

$$
\mathbf{Z}_{Q}(s)=\underbrace{\frac{s-a_{33}}{s^{4}+p_{3} s^{3}+p_{2} s^{2}+p_{1} s+p_{0}}}_{H(s)}\left[\begin{array}{cc}
Z_{11}(s) & Z_{12}(s) \\
Z_{21}(s) & Z_{22}(s)
\end{array}\right]
$$

where

$$
\begin{aligned}
& Z_{11}(s) \triangleq s^{2}-\left(a_{22}+a_{33}\right) s+a_{22} a_{33}-a_{23} a_{32}, \\
& Z_{12}(s) \triangleq a_{12} s-a_{12} a_{33}+a_{13} a_{32}, \\
& Z_{21}(s) \triangleq a_{21} s-a_{21} a_{33}+a_{23} a_{31}, \\
& Z_{22}(s) \triangleq s^{2}-\left(a_{11}+a_{33}\right) s+a_{11} a_{33}-a_{13} a_{31} .
\end{aligned}
$$

It follows from Eq. (176) that any zero of $H(s)$ which is not also a zero of the numerator terms in Eq. (176) is a pole of $\mathbf{Z}_{Q}(s)$. Since Eq. (170) is a third-order system, we expect $\mathbf{Z}_{Q}(s)$ to have three poles. This implies that one zero of $H(s)$ in Eq. (176) must also be present in the numerator in order that they cancel each other out. Substituting $s=i \omega$ in Eq. (176) and calculating the Hermitian matrix $\mathbf{Z}_{Q}^{H}(i \omega)$, we obtain

$$
\begin{aligned}
\mathbf{Z}_{Q}(i \omega)+\mathbf{Z}_{Q}^{\dagger}(i \omega) & =H(i \omega)\left[\begin{array}{ll}
Z_{11}(i \omega) & Z_{12}(i \omega) \\
Z_{21}(i \omega) & Z_{22}(i \omega)
\end{array}\right]+H(-i \omega)\left[\begin{array}{cc}
Z_{11}(-i \omega) & Z_{21}(-i \omega) \\
Z_{12}(-i \omega) & Z_{22}(-i \omega)
\end{array}\right] \\
& =\left[\begin{array}{cc}
H(i \omega) Z_{11}(i \omega)+H(-i \omega) Z_{11}(-i \omega) & H(i \omega) Z_{12}(i \omega)+H(-i \omega) Z_{21}(-i \omega) \\
H(-i \omega) Z_{12}(-i \omega)+H(i \omega) Z_{21}(i \omega) & H(i \omega) Z_{22}(i \omega)+H(-i \omega) Z_{22}(-i \omega)
\end{array}\right] \\
& =\left[\begin{array}{cc}
2 \operatorname{Re}\left[H(i \omega) Z_{11}(i \omega)\right] & H(i \omega) Z_{12}(i \omega)+H(-i \omega) Z_{21}(-i \omega) \\
H(-i \omega) Z_{12}(-i \omega)+H(i \omega) Z_{21}(i \omega) & 2 \operatorname{Re}\left[H(i \omega) Z_{22}(i \omega)\right]
\end{array}\right]
\end{aligned}
$$

where $H(i \omega)$ is the scalar polynomial defined in Eq. (176). Let us calculate next

$$
\begin{aligned}
\operatorname{Re}\left[H(i \omega) Z_{11}(i \omega)\right]= & \frac{-a_{33}\left(\omega^{4}-p_{2} \omega^{2}+p_{0}\right)+\omega\left(p_{3} \omega^{3}+p_{1} \omega\right)}{\left(\omega^{4}-p_{2} \omega^{2}+p_{0}\right)^{2}+\left(p_{3} \omega^{3}+p_{1} \omega\right)^{2}} \operatorname{Re}\left[Z_{11}(i \omega)\right] \\
& +\frac{\omega\left(\omega^{4}-p_{2} \omega^{2}+p_{0}\right)+a_{33}\left(p_{3} \omega^{3}+p_{1} \omega\right)}{\left(\omega^{4}-p_{2} \omega^{2}+p_{0}\right)^{2}+\left(p_{3} \omega^{3}+p_{1} \omega\right)^{2}} \operatorname{Im}\left[Z_{11}(i \omega)\right] .
\end{aligned}
$$

\subsection{Local passive regions obtained from $\mathrm{Y}_{Q}(s)$}

Let us derive the local passive regions directly from $\mathbf{Y}_{Q}(s) \triangleq \mathbf{Z}_{Q}^{-1}(s)$; namely, from the $2 \times 2$ matrix in the top row of Eq. (173):

$$
\begin{aligned}
\mathbf{Y}_{Q}(s) & =\left[\begin{array}{cc}
s-a_{11}-\frac{a_{13} a_{31}}{s-a_{33}} & -a_{12}-\frac{a_{13} a_{32}}{s-a_{33}} \\
-a_{21}-\frac{a_{23} a_{31}}{s-a_{33}} & s-a_{22}-\frac{a_{23} a_{32}}{s-a_{33}}
\end{array}\right] \\
& =\frac{1}{s-a_{33}}\left[\begin{array}{cc}
\left(s-a_{11}\right)\left(s-a_{33}\right)-a_{13} a_{31} & -a_{12}\left(s-a_{33}\right)-a_{13} a_{32} \\
-a_{21}\left(s-a_{33}\right)-a_{23} a_{31} & \left(s-a_{22}\right)\left(s-a_{33}\right)-a_{23} a_{32}
\end{array}\right] .
\end{aligned}
$$




\subsection{1. $\mathrm{P} 1\left(\mathbf{Y}_{Q}(s)\right)$}

Since $\mathbf{Y}_{Q}(s)$ has only one finite pole $s=a_{33}$, the condition for $\mathrm{P} 1\left(\mathbf{Y}_{Q}(s)\right)$ is given simply by $a_{33} \leq 0$. For the pole at infinity we have the residue matrix

$$
\lim _{\omega \rightarrow \infty} \frac{\mathbf{Y}_{Q}(i \omega)}{i \omega}=\left[\begin{array}{ll}
1 & 0 \\
0 & 1
\end{array}\right]
$$

which is Hermitian and positive definite. Therefore the parameter region which satisfies $\mathrm{P} 1\left(\mathbf{Y}_{Q}(s)\right)$ is given by

$$
a_{33} \leq 0
$$

\subsection{2. $\mathrm{P} 2\left(\mathbf{Y}_{Q}(s)\right)$}

$$
\begin{aligned}
& \mathbf{Y}_{Q}^{H}(i \omega)=\mathbf{Y}_{Q}^{\dagger}(i \omega)+\mathbf{Y}_{Q}(i \omega) \\
& =\left[\begin{array}{cc}
-i \omega-a_{11}-\frac{a_{13} a_{31}}{-i \omega-a_{33}} & -a_{21}-\frac{a_{23} a_{31}}{-i \omega-a_{33}} \\
-a_{12}-\frac{a_{13} a_{32}}{-i \omega-a_{33}} & -i \omega-a_{22}-\frac{a_{23} a_{32}}{-i \omega-a_{33}}
\end{array}\right]+\left[\begin{array}{cc}
i \omega-a_{11}-\frac{a_{13} a_{31}}{i \omega-a_{33}} & -a_{12}-\frac{a_{13} a_{32}}{i \omega-a_{33}} \\
-a_{21}-\frac{a_{23} a_{31}}{i \omega-a_{33}} & i \omega-a_{22}-\frac{a_{23} a_{32}}{i \omega-a_{33}}
\end{array}\right] \\
& =\left[\begin{array}{cc}
-2 a_{11}+\frac{2 a_{13} a_{31} a_{33}}{\omega^{2}+a_{33}^{2}} & -a_{21}-a_{12}-\frac{a_{23} a_{31}}{-i \omega-a_{33}}-\frac{a_{13} a_{32}}{i \omega-a_{33}} \\
-a_{21}-a_{12}-\frac{a_{23} a_{31}}{i \omega-a_{33}}-\frac{a_{13} a_{32}}{-i \omega-a_{33}} & -2 a_{22}+\frac{2 a_{23} a_{32} a_{33}}{\omega^{2}+a_{33}^{2}}
\end{array}\right] \\
& =\left[\begin{array}{cc}
-2 a_{11}+\frac{2 a_{13} a_{31} a_{33}}{\omega^{2}+a_{33}^{2}} & -a_{21}-a_{12}-\frac{a_{23} a_{31}\left(i \omega-a_{33}\right)+a_{13} a_{32}\left(-i \omega-a_{33}\right)}{\omega^{2}+a_{33}^{2}} \\
-a_{21}-a_{12}-\frac{a_{23} a_{31}\left(-i \omega-a_{33}\right)+a_{13} a_{32}\left(i \omega-a_{33}\right)}{\omega^{2}+a_{33}^{2}} & -2 a_{22}+\frac{2 a_{23} a_{32} a_{33}}{\omega^{2}+a_{33}^{2}}
\end{array}\right] .
\end{aligned}
$$

Since $\mathbf{Y}_{Q}^{H}(i \omega)$ is a Hermitian matrix, we know that all its eigenvalues are real. Since a Hermitian matrix is positive semi-definite if, and only if, all its eigenvalues are non-negative (Theorem 1), it follows that the condition $\mathrm{P} 2\left(\mathbf{Y}_{Q}(s)\right)$ is equivalent to deriving conditions such that all eigenvalues of $\mathbf{Y}_{Q}^{H}(i \omega)$ are non-negative for all $\omega \in \mathbb{R}$. The eigenvalues of $\mathbf{Y}_{Q}^{H}(i \omega)$ are the solutions of

$$
\left|\begin{array}{cc}
\lambda+2 a_{11}-\frac{2 a_{13} a_{31} a_{33}}{\omega^{2}+a_{33}^{2}} & a_{21}+a_{12}+\frac{a_{23} a_{31}\left(i \omega-a_{33}\right)+a_{13} a_{32}\left(-i \omega-a_{33}\right)}{\omega^{2}+a_{33}^{2}} \\
a_{21}+a_{12}+\frac{a_{23} a_{31}\left(-i \omega-a_{33}\right)+a_{13} a_{32}\left(i \omega-a_{33}\right)}{\omega^{2}+a_{33}^{2}} & \lambda+2 a_{22}-\frac{2 a_{23} a_{32} a_{33}}{\omega^{2}+a_{33}^{2}}
\end{array}\right|=0
$$

from which we have

$$
\left|\begin{array}{cc}
\lambda+2 a_{11}-\frac{2 a_{13} a_{31} a_{33}}{\omega^{2}+a_{33}^{2}} & a_{21}+a_{12}+\frac{-a_{33}\left(a_{23} a_{31}+a_{13} a_{32}\right)+i \omega\left(a_{23} a_{31}-a_{13} a_{32}\right)}{\omega^{2}+a_{33}^{2}} \\
a_{21}+a_{12}+\frac{-a_{33}\left(a_{23} a_{31}+a_{13} a_{32}\right)-i \omega\left(a_{23} a_{31}-a_{13} a_{32}\right)}{\omega^{2}+a_{33}^{2}} & \lambda+2 a_{22}-\frac{2 a_{23} a_{32} a_{33}}{\omega^{2}+a_{33}^{2}}
\end{array}\right|=0
$$


and

$$
\begin{aligned}
P(\lambda) \triangleq & \lambda^{2}+2\left(a_{11}+a_{22}-\frac{\left(a_{13} a_{31}+a_{23} a_{32}\right) a_{33}}{\omega^{2}+a_{33}^{2}}\right) \lambda+4 a_{11} a_{22}+\frac{-4 a_{33}}{\omega^{2}+a_{33}^{2}}\left(a_{11} a_{23} a_{32}+a_{22} a_{13} a_{31}\right) \\
& +\frac{4 a_{13} a_{31} a_{23} a_{32} a_{33}^{2}}{\left(\omega^{2}+a_{33}^{2}\right)^{2}}-\left(a_{21}+a_{12}-\frac{\left(a_{23} a_{31}+a_{13} a_{32}\right) a_{33}}{\omega^{2}+a_{33}^{2}}\right)^{2}-\frac{\omega^{2}\left(a_{23} a_{31}-a_{13} a_{32}\right)^{2}}{\left(\omega^{2}+a_{33}^{2}\right)^{2}}=0 .
\end{aligned}
$$

Let $P\left(\lambda_{\min }\right)$ be the minimum value of $P(\lambda)$, then the condition "both solutions $\lambda_{1,2}$ of $P(\lambda)$ are nonnegative" is equivalent to the condition

$$
\lambda_{\min } \geq 0 \quad \mathbb{A N D} \quad P(0) \geq 0
$$

where $\lambda_{\min }$ denotes the solution of the equation

$$
\frac{d P(\lambda)}{d \lambda}=2 \lambda+2\left(a_{11}+a_{22}-\frac{\left(a_{13} a_{31}+a_{23} a_{32}\right) a_{33}}{\omega^{2}+a_{33}^{2}}\right)=0
$$

namely,

$$
\lambda_{\min }=-\left(a_{11}+a_{22}-\frac{\left(a_{13} a_{31}+a_{23} a_{32}\right) a_{33}}{\omega^{2}+a_{33}^{2}}\right) .
$$

Substituting $\lambda=0$ in Eq. (185), we obtain

$$
\begin{aligned}
P(0)= & 4 a_{11} a_{22}+\frac{-4 a_{33}}{\omega^{2}+a_{33}^{2}}\left(a_{11} a_{23} a_{32}+a_{22} a_{13} a_{31}\right)+\frac{4 a_{13} a_{31} a_{23} a_{32} a_{33}^{2}}{\left(\omega^{2}+a_{33}^{2}\right)^{2}} \\
& -\left(a_{21}+a_{12}-\frac{\left(a_{23} a_{31}+a_{13} a_{32}\right) a_{33}}{\omega^{2}+a_{33}^{2}}\right)^{2}-\frac{\omega^{2}\left(a_{23} a_{31}-a_{13} a_{32}\right)^{2}}{\left(\omega^{2}+a_{33}^{2}\right)^{2}} .
\end{aligned}
$$

\section{Parameter ranges}

$\mathrm{P} 2\left(Y_{Q}(s)\right)$ is equivalent to the condition

$$
\begin{gathered}
\left(a_{11}+a_{22}-\frac{\left(a_{13} a_{31}+a_{23} a_{32}\right) a_{33}}{\omega^{2}+a_{33}^{2}}\right) \leq 0 \quad \mathbb{A N D} \quad 4 a_{11} a_{22}+\frac{-4 a_{33}}{\omega^{2}+a_{33}^{2}}\left(a_{11} a_{23} a_{32}+a_{22} a_{13} a_{31}\right) \\
+\frac{4 a_{13} a_{31} a_{23} a_{32} a_{33}^{2}}{\left(\omega^{2}+a_{33}^{2}\right)^{2}}-\left(a_{21}+a_{12}-\frac{\left(a_{23} a_{31}+a_{13} a_{32}\right) a_{33}}{\omega^{2}+a_{33}^{2}}\right)^{2}-\frac{\omega^{2}\left(a_{23} a_{31}-a_{13} a_{32}\right)^{2}}{\left(\omega^{2}+a_{33}^{2}\right)^{2}} \geq 0 .
\end{gathered}
$$

Observe that Eq. (190) contains two sets of conditions. From the first condition in Eq. (190) we have

$$
\left(a_{11}+a_{22}\right)\left(\omega^{2}+a_{33}^{2}\right) \leq\left(a_{13} a_{31}+a_{23} a_{32}\right) a_{33} .
$$

Since this inequality must hold for all $\omega$, therefore we have

$$
a_{11}+a_{22} \leq 0 \quad \mathbb{A N D} \quad\left(a_{13} a_{31}+a_{23} a_{32}\right) a_{33} \geq\left(a_{11}+a_{22}\right) a_{33}^{2} .
$$

From the second condition in Eq. (190) we have

$$
\begin{gathered}
4 a_{11} a_{22}\left(\omega^{2}+a_{33}^{2}\right)^{2}-4 a_{33}\left(\omega^{2}+a_{33}^{2}\right)\left(a_{11} a_{23} a_{32}+a_{22} a_{13} a_{31}\right)+4 a_{13} a_{31} a_{23} a_{32} a_{33}^{2} \\
-\left[\left(a_{21}+a_{12}\right)\left(\omega^{2}+a_{33}^{2}\right)-\left(a_{23} a_{31}+a_{13} a_{32}\right) a_{33}\right]^{2}-\omega^{2}\left(a_{23} a_{31}-a_{13} a_{32}\right)^{2} \geq 0
\end{gathered}
$$


which is equivalent to

$$
\begin{gathered}
4 a_{11} a_{22}\left(\omega^{4}+2 \omega^{2} a_{33}^{2}+a_{33}^{4}\right)-4 a_{33}\left(\omega^{2}+a_{33}^{2}\right)\left(a_{11} a_{23} a_{32}+a_{22} a_{13} a_{31}\right)+4 a_{13} a_{31} a_{23} a_{32} a_{33}^{2} \\
-\left[\left(a_{21}+a_{12}\right) \omega^{2}+a_{33}^{2}\left(a_{21}+a_{12}\right)-\left(a_{23} a_{31}+a_{13} a_{32}\right) a_{33}\right]^{2}-\omega^{2}\left(a_{23} a_{31}-a_{13} a_{32}\right)^{2} \geq 0 .
\end{gathered}
$$

Let us rearrange the items in Eq. (194) in the decreasing order of $\omega$ as follow:

$$
\begin{aligned}
A(\omega) \triangleq & {\left[4 a_{11} a_{22}-\left(a_{21}+a_{12}\right)^{2}\right] \omega^{4}+\left\{8 a_{11} a_{22} a_{33}^{2}-4 a_{33}\left(a_{11} a_{23} a_{32}+a_{22} a_{13} a_{31}\right)-\left(a_{23} a_{31}-a_{13} a_{32}\right)^{2}\right.} \\
& \left.-2\left(a_{21}+a_{12}\right)\left[a_{33}^{2}\left(a_{21}+a_{12}\right)-\left(a_{23} a_{31}+a_{13} a_{32}\right) a_{33}\right]\right\} \omega^{2}+\left[4 a_{11} a_{22} a_{33}^{4}\right. \\
& \left.-4 a_{33}^{3}\left(a_{11} a_{23} a_{32}+a_{22} a_{13} a_{31}\right)+4 a_{13} a_{31} a_{23} a_{32} a_{33}^{2}\right]-\left[a_{33}^{2}\left(a_{21}+a_{12}\right)\right. \\
& \left.-\left(a_{23} a_{31}+a_{13} a_{32}\right) a_{33}\right]^{2} \geq 0 .
\end{aligned}
$$

Let us recast $A(\omega)$ as follow:

$$
A(\omega)=A_{4} \omega^{4}+A_{2} \omega^{2}+A_{0}
$$

where

$$
\begin{aligned}
A_{4} \triangleq & 4 a_{11} a_{22}-\left(a_{21}+a_{12}\right)^{2} \\
A_{2} \triangleq & \left\{8 a_{11} a_{22} a_{33}^{2}-4 a_{33}\left(a_{11} a_{23} a_{32}+a_{22} a_{13} a_{31}\right)-\left(a_{23} a_{31}-a_{13} a_{32}\right)^{2}-2\left(a_{21}+a_{12}\right)\left[a_{33}^{2}\left(a_{21}+a_{12}\right)\right.\right. \\
& \left.\left.-\left(a_{23} a_{31}+a_{13} a_{32}\right) a_{33}\right]\right\} \\
A_{0} \triangleq & {\left[4 a_{11} a_{22} a_{33}^{4}-4 a_{33}^{3}\left(a_{11} a_{23} a_{32}+a_{22} a_{13} a_{31}\right)+4 a_{13} a_{31} a_{23} a_{32} a_{33}^{2}\right]-\left[a_{33}^{2}\left(a_{21}+a_{12}\right)\right.} \\
& \left.-\left(a_{23} a_{31}+a_{13} a_{32}\right) a_{33}\right]^{2} .
\end{aligned}
$$

Observe that $A(\omega) \geq 0$ for any $\omega \in \mathbb{R}$ is equivalent to the condition

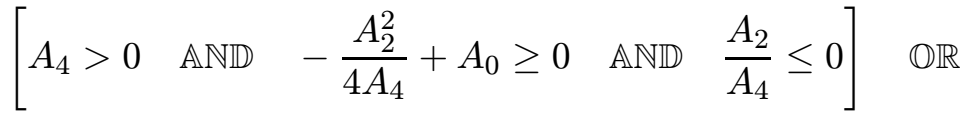

$$
\begin{aligned}
& {\left[\begin{array}{lllll}
A_{4}>0 & \mathbb{A N D} & A_{0} \geq 0 & \mathbb{A N D} & \frac{A_{2}}{A_{4}}>0
\end{array}\right] \quad \mathbb{O} \mathbb{R}} \\
& {\left[A_{4}=0 \quad \mathbb{A N D} \quad A_{2} \geq 0 \quad \mathbb{A N D} \quad A_{0} \geq 0\right]}
\end{aligned}
$$

which is equivalent to

$$
\begin{aligned}
& {\left[\begin{array}{lllll}
A_{4}>0 & \mathbb{A N D} & -\frac{A_{2}^{2}}{4 A_{4}}+A_{0} \geq 0 & \mathbb{A N D} & A_{2} \leq 0
\end{array}\right] \quad \mathbb{O} \mathbb{R} \quad\left[\begin{array}{lllll}
A_{4}>0 & \mathbb{A N D} & A_{0} \geq 0 & \mathbb{A N D} & A_{2}>0
\end{array}\right]} \\
& \mathbb{O} R \quad\left[\begin{array}{lllll}
A_{4}=0 & \mathbb{A N D} & A_{2} \geq 0 & \mathbb{A N D} & A_{0} \geq 0
\end{array}\right] .
\end{aligned}
$$

Equation (199) can be simplified to

$$
\begin{aligned}
& \left\{\begin{array}{lll}
A_{4}>0 & \mathbb{A N D}
\end{array}\left[\left(-\frac{A_{2}^{2}}{4 A_{4}}+A_{0} \geq 0 \quad \mathbb{A N D} \quad A_{2} \leq 0\right) \quad \mathbb{O} \mathbb{R} \quad\left(\begin{array}{lll}
A_{0} \geq 0 & \mathbb{A N D} & A_{2}>0
\end{array}\right)\right]\right\} \quad \mathbb{O R} \\
& {\left[A_{4}=0 \quad \mathbb{A N D} \quad A_{2} \geq 0 \quad \mathbb{A N D} \quad A_{0} \geq 0\right] .}
\end{aligned}
$$

Let us apply Theorem 2. The first condition in Theorem 2 leads to

$$
-2 a_{11}+\frac{2 a_{13} a_{31} a_{33}}{\omega^{2}+a_{33}^{2}} \geq 0
$$


from which we have

$$
a_{13} a_{31} a_{33} \geq a_{11}\left(\omega^{2}+a_{33}^{2}\right), \quad \forall \omega \in \mathbb{R}
$$

which leads to

$$
a_{11} \leq 0 \quad \mathbb{A N D} \quad a_{13} a_{31} a_{33} \geq a_{11} a_{33}^{2}
$$

From the second condition of Theorem 2, we get the same set of results as the second condition in Eq. (190). Therefore no new information is obtained.

It follows from the above analysis that $P 2\left(\mathbf{Y}_{Q}(s)\right)$ is equivalent to the following condition:

$$
\begin{aligned}
& a_{11}+a_{22} \leq 0 \quad \mathbb{A N D} \quad\left(a_{13} a_{31}+a_{23} a_{32}\right) a_{33} \geq\left(a_{11}+a_{22}\right) a_{33}^{2} \quad \mathbb{A N D} \\
& \left\{\left\{A_{4}>0 \quad \mathbb{A N D}\left[\left(-\frac{A_{2}^{2}}{4 A_{4}}+A_{0} \geq 0 \quad \mathbb{A N D} \quad A_{2} \leq 0\right) \quad \mathbb{O} \mathbb{R} \quad\left(\begin{array}{lll}
A_{0} \geq 0 & \mathbb{A N D} & A_{2}>0
\end{array}\right)\right]\right\} \quad \mathbb{O R}\right. \\
& \left.\left[A_{4}=0 \quad \mathbb{A N D} \quad A_{2} \geq 0 \quad \mathbb{A N D} \quad A_{0} \geq 0\right]\right\} \quad \mathbb{A N D} \quad a_{11} \leq 0 \quad \mathbb{A N D} \quad a_{13} a_{31} a_{33} \geq a_{11} a_{33}^{2} .
\end{aligned}
$$

\subsection{3. $\mathrm{P} 3\left(\mathbf{Y}_{Q}(s)\right)$}

There is only a simple pole when $a_{33}=0$. When $a_{33}=0$ the residue matrix is given by

$$
\mathbf{K}_{-1}=\lim _{s \rightarrow 0} s \mathbf{Y}_{Q}(s)=\left[\begin{array}{ll}
-a_{13} a_{31} & -a_{13} a_{32} \\
-a_{23} a_{31} & -a_{23} a_{32}
\end{array}\right] \text {. }
$$

The above matrix is a Hermitian matrix if

$$
a_{13} a_{32}=a_{23} a_{31} .
$$

\section{Parameter ranges}

It follows that $\mathrm{P} 3\left(\mathbf{Y}_{Q}(s)\right)$ is satisfied in the following parameter regions:

$$
a_{33}=0 \quad \mathbb{A N D} \quad a_{13} a_{32}=a_{23} a_{31}
$$

and $\mathbf{K}$ is positive semi-definite.

\section{Parameter Regions for Local Passivity}

The parameter region for local passivity is given by:

$$
\mathrm{P} 1\left(\mathbf{Y}_{Q}(s)\right) \cap \mathrm{P} 2\left(\mathbf{Y}_{Q}(s)\right) \cap \mathrm{P} 3\left(\mathbf{Y}_{Q}(s)\right)
$$

\section{Parameter Regions for Local Activity}

The parameter region for local passivity is given by:

$$
\mathbb{N O T}\left[\mathrm{P} 1\left(\mathbf{Y}_{Q}(s)\right) \cap \mathrm{P} 2\left(\mathbf{Y}_{Q}(s)\right) \cap \mathrm{P} 3\left(\mathbf{Y}_{Q}(s)\right)\right]
$$

\section{Parameter Regions for Edge of Chaos}

The parameter region for edge of chaos is given by:

$$
\mathbb{N O T}\left[\mathrm{P} 1\left(\mathbf{Y}_{Q}(s)\right) \cap \mathrm{P} 2\left(\mathbf{Y}_{Q}(s)\right) \cap \mathrm{P} 3\left(\mathbf{Y}_{Q}(s)\right)\right] \cap
$$

[Poles of $\mathbf{Z}_{Q}(s)$ in the left-half $s$-plane]

\section{Three-Port and Third-Order CNN Cells}

Let us consider next the case of three-port thirdorder CNN cells. The linearized cell state equation at an equilibrium point is given by

$$
\begin{aligned}
& \dot{v}_{1}=a_{11} v_{1}+a_{12} v_{2}+a_{13} v_{3}+i_{1}, \\
& \dot{v}_{2}=a_{21} v_{1}+a_{22} v_{2}+a_{23} v_{3}+i_{2}, \\
& \dot{v}_{3}=a_{31} v_{1}+a_{32} v_{2}+a_{33} v_{3}+i_{3} .
\end{aligned}
$$

Applying Laplace transform to Eq. (208), we obtain the admittance matrix

$$
\mathbf{Y}_{Q}(s)=\left[\begin{array}{ccc}
s-a_{11} & -a_{12} & -a_{13} \\
-a_{21} & s-a_{22} & -a_{23} \\
-a_{31} & -a_{32} & s-a_{33}
\end{array}\right]
$$


The corresponding impedance matrix is, by definition,

$$
\begin{aligned}
\mathbf{Z}_{Q}(s) \triangleq \mathbf{Y}_{Q}^{-1}(s) & =\left[\begin{array}{ccc}
s-a_{11} & -a_{12} & -a_{13} \\
-a_{21} & s-a_{22} & -a_{23} \\
-a_{31} & -a_{32} & s-a_{33}
\end{array}\right]^{-1} \\
& =\frac{1}{P(s)}\left[\begin{array}{ccc}
Z_{11} & Z_{12} & Z_{13} \\
Z_{21} & Z_{22} & Z_{23} \\
Z_{31} & Z_{32} & Z_{33}
\end{array}\right] \quad(210)
\end{aligned}
$$

where

$$
\begin{aligned}
P(s) \triangleq & \left(s-a_{11}\right)\left[\left(s-a_{22}\right)\left(s-a_{33}\right)\right. \\
& \left.-a_{23} a_{32}\right]+a_{21}\left[-a_{12}\left(s-a_{33}\right)\right. \\
& \left.-a_{13} a_{32}\right]-a_{31}\left[a_{12} a_{23}+a_{13}\left(s-a_{22}\right)\right] \\
= & s^{3}+\left(-a_{22}-a_{33}-a_{11}\right) s^{2}+\left[a_{22} a_{33}\right. \\
& -a_{23} a_{32}+a_{11}\left(a_{22}+a_{33}\right)-a_{12} a_{21} \\
& \left.-a_{13} a_{31}\right] s+\left(-a_{11} a_{22} a_{33}-a_{11} a_{23} a_{32}\right. \\
& +a_{12} a_{21} a_{33}-a_{13} a_{21} a_{32}-a_{12} a_{23} a_{31} \\
& \left.+a_{13} a_{22} a_{31}\right) .
\end{aligned}
$$

The roots of $P(s)$ in Eq. (211) are the poles of $\mathbf{Z}_{Q}(s)$. It is not necessary to find the values of $Z_{i j}$ in Eq. (210), $i, j=1,2,3$, because only poles of $\mathbf{Z}_{Q}(s)$ are needed and they do not depend on $Z_{i j}$.

\subsection{Local passive regions from $\mathrm{Y}_{Q}(s)$}

\subsection{1. $\mathrm{P} 1\left(\mathbf{Y}_{Q}(s)\right)$}

Since $\mathbf{Y}_{Q}(s)$ has no finite poles, it suffices to consider only the pole at infinity, whose residue-matrix

$$
\lim _{\omega \rightarrow \infty} \frac{\mathbf{Y}_{Q}(i \omega)}{i \omega}=\left[\begin{array}{lll}
1 & 0 & 0 \\
0 & 1 & 0 \\
0 & 0 & 1
\end{array}\right]
$$

is Hermitian and positive definite.

\subsection{2. $\mathrm{P} 2\left(\mathbf{Y}_{Q}(s)\right)$}

$$
\begin{aligned}
\mathbf{Y}_{Q}^{H}(i \omega)=\mathbf{Y}_{Q}^{\dagger}(i \omega)+\mathbf{Y}_{Q}(i \omega) & =\left[\begin{array}{ccc}
-i \omega-a_{11} & -a_{21} & -a_{31} \\
-a_{12} & -i \omega-a_{22} & -a_{32} \\
-a_{13} & -a_{23} & -i \omega-a_{33}
\end{array}\right] \\
& +\left[\begin{array}{ccc}
i \omega-a_{11} & -a_{12} & -a_{13} \\
-a_{21} & i \omega-a_{22} & -a_{23} \\
-a_{31} & -a_{32} & i \omega-a_{33}
\end{array}\right] \\
= & {\left[\begin{array}{ccc}
-2 a_{11} & -\left(a_{12}+a_{21}\right) & -\left(a_{13}+a_{31}\right) \\
-\left(a_{21}+a_{12}\right) & -2 a_{22} & -\left(a_{23}+a_{32}\right) \\
-\left(a_{31}+a_{13}\right) & -\left(a_{32}+a_{23}\right) & -2 a_{33}
\end{array}\right] . }
\end{aligned}
$$

$\mathbf{Y}_{Q}^{H}(i \omega)$ is positive semi-definite if, and only if, the following conditions are satisfied:

1. $-2 a_{11} \geq 0, \quad \mathbb{A N D}$

2. $\left|\begin{array}{cc}-2 a_{11} & -\left(a_{12}+a_{21}\right) \\ -\left(a_{21}+a_{12}\right) & -2 a_{22}\end{array}\right| \geq 0, \quad \mathbb{A N D}$

3. $-2 a_{11}\left|\begin{array}{cc}-2 a_{22} & -\left(a_{23}+a_{32}\right) \\ -\left(a_{32}+a_{23}\right) & -2 a_{33}\end{array}\right|$

$$
\begin{gathered}
+\left(a_{21}+a_{12}\right)\left|\begin{array}{cc}
-\left(a_{12}+a_{21}\right) & -\left(a_{13}+a_{31}\right) \\
-\left(a_{32}+a_{23}\right) & -2 a_{33}
\end{array}\right| \\
-\left(a_{31}+a_{13}\right)\left|\begin{array}{cc}
-\left(a_{12}+a_{21}\right) & -\left(a_{13}+a_{31}\right) \\
-2 a_{22} & -\left(a_{23}+a_{32}\right)
\end{array}\right| \geq 0 .
\end{gathered}
$$

From condition 2 of Eq. (214) we have

$$
4 a_{11} a_{22}-\left(a_{12}+a_{21}\right)^{2} \geq 0 .
$$

From condition 3 of Eq. (214) we have

$$
\begin{aligned}
-2 a_{11} & {\left[4 a_{22} a_{33}-\left(a_{23}+a_{32}\right)^{2}\right] } \\
& +\left(a_{12}+a_{21}\right)\left[2 a_{33}\left(a_{12}+a_{21}\right)\right. \\
& \left.-\left(a_{13}+a_{31}\right)\left(a_{32}+a_{23}\right)\right] \\
& -\left(a_{13}+a_{31}\right)\left[\left(a_{12}+a_{21}\right)\left(a_{23}+a_{32}\right)\right. \\
& \left.-2 a_{22}\left(a_{13}+a_{31}\right)\right] \geq 0 .
\end{aligned}
$$


Therefore $P 2\left(\mathbf{Y}_{Q}(s)\right)$ is equivalent to the condition

$$
\begin{aligned}
a_{11} \leq & 0 \quad \mathbb{A N D} \quad 4 a_{11} a_{22}-\left(a_{12}+a_{21}\right)^{2} \geq 0 \\
& \mathbb{A} \mathbb{N D} \\
& -2 a_{11}\left[4 a_{22} a_{33}-\left(a_{23}+a_{32}\right)^{2}\right] \\
& +\left(a_{12}+a_{21}\right)\left[2 a_{33}\left(a_{12}+a_{21}\right)\right. \\
& \left.-\left(a_{13}+a_{31}\right)\left(a_{32}+a_{23}\right)\right] \\
& -\left(a_{13}+a_{31}\right)\left[\left(a_{12}+a_{21}\right)\left(a_{23}+a_{32}\right)\right. \\
& \left.-2 a_{22}\left(a_{13}+a_{31}\right)\right] \geq 0
\end{aligned}
$$

\subsection{3. $\mathrm{P} 3\left(\mathbf{Y}_{Q}(s)\right)$}

$\mathbf{Y}_{Q}(s)$ has an infinite pole (i.e. a simple pole at infinity) on the imaginary axis. In this case, the associated residue matrix is given by

$$
\mathbf{K}_{-1}=\lim _{\omega \rightarrow \infty} \frac{\mathbf{Y}_{Q}(i \omega)}{i \omega}=\left[\begin{array}{lll}
1 & 0 & 0 \\
0 & 1 & 0 \\
0 & 0 & 1
\end{array}\right]
$$

which is Hermitian and positive definite.

\section{Remarks}

Similarly, we can find the locally passive and active regions by using $\mathrm{P} 3\left(\mathbf{Y}_{Q}(s)\right), \mathrm{P} 2\left(\mathbf{Y}_{Q}(s)\right)$ and $\mathrm{P} 3\left(\mathbf{Y}_{Q}(s)\right)$. The edge-of-chaos regions can be found from the local active region and the poles of $\mathbf{Z}_{Q}(s)$.

\section{CNN Made of Smooth Chua's Circuits}

In general, it is difficult to derive analytical criteria for two-port third-order CNN cells. However, the special structure of the Chua's circuit equation with a cubic nonlinearity makes it possible to investigate the local passive and the local active parameter regions analytically. A cubic Chua's circuit two-port CNN cell is defined by

$$
\begin{aligned}
& \dot{V}_{1}=\alpha\left[V_{2}-a V_{1}^{3}-c V_{1}\right]+\alpha I_{1}^{*}, \\
& \dot{V}_{2}=V_{1}-V_{2}+V_{3}+I_{2}^{*}, \\
& \dot{V}_{3}=-\beta V_{2}-\gamma V_{3} .
\end{aligned}
$$

The cell equilibrium points for a given pair of port currents $\left(I_{1}^{*}, I_{2}^{*}\right)$ representing the external cell couplings are given by

$$
\begin{gathered}
-a V_{1}^{* 3}+\left(-c+\frac{\gamma}{\beta+\gamma}\right) V_{1}^{*}+I_{1}^{*} \\
+\frac{\gamma}{\beta+\gamma} I_{2}^{*}=0, \\
V_{2}^{*}=\frac{\gamma}{\beta+\gamma}\left(V_{1}^{*}+I_{2}^{*}\right), \\
V_{3}^{*}=-\frac{\beta}{\gamma} V_{2}^{*} .
\end{gathered}
$$

Defining

$$
\begin{gathered}
I^{*} \triangleq\left(I_{1}^{*}+\frac{\gamma}{\beta+\gamma} I_{2}^{*}\right) / a, \\
\Lambda \triangleq\left(-c+\frac{\gamma}{\beta+\gamma}\right) / a
\end{gathered}
$$

we can rewrite Eq. (220) as follow:

$$
\begin{aligned}
V_{1}^{* 3}-\Lambda V_{1}^{*}-I^{*} & =0 \\
V_{2}^{*} & =\frac{\gamma}{\beta+\gamma}\left(V_{1}^{*}+I_{2}^{*}\right), \\
V_{3}^{*} & =-\frac{\beta}{\gamma} V_{2}^{*} .
\end{aligned}
$$

Depending on the value of

$$
\mathfrak{D} \triangleq \frac{I^{* 2}}{4}-\frac{\Lambda^{3}}{27}
$$

a Chua's circuit CNN cell can have one, two or three equilibrium points:

1. $\mathfrak{D}>0$, Chua's circuit has only one equilibrium point given by

$$
v_{1}^{*}=\left(\frac{I^{*}}{2}+\sqrt{\mathfrak{D}}\right)^{1 / 3}+\left(\frac{I^{*}}{2}-\sqrt{\mathfrak{D}}\right)^{1 / 3} .
$$

2. $\mathfrak{D}=0$, Chua's circuit has two equilibrium points given by

$$
v_{1 a}^{*}=2\left(\frac{I^{*}}{2}\right)^{1 / 3}, v_{1 b}^{*}=\left(\frac{I^{*}}{2}\right)^{1 / 3} .
$$

3. $\mathfrak{D}<0$, Chua's circuit has three equilibrium points given by

$$
\begin{aligned}
& v_{1 a}^{*}=2 \sqrt{\frac{|\Lambda|}{3}} \cos \frac{\psi}{3}, \\
& v_{1 b}^{*}=-2 \sqrt{\frac{|\Lambda|}{3}} \cos (\psi / 3-\pi / 3), \\
& v_{1 c}^{*}=-2 \sqrt{\frac{|\Lambda|}{3}} \cos (\psi / 3+\pi / 3)
\end{aligned}
$$


where

$$
\psi=\cos ^{-1}\left(\frac{I^{*} / 2}{\sqrt{|\Lambda|^{3} / 27}}\right) .
$$

The Jacobian matrix of the linearized cell state equation at each equilibrium point is given by

$$
\begin{aligned}
J_{Q} & \triangleq\left[\begin{array}{lll}
a_{11} & a_{12} & a_{13} \\
a_{21} & a_{22} & a_{23} \\
a_{31} & a_{32} & a_{33}
\end{array}\right] \\
& =\left[\begin{array}{ccc}
-\alpha\left(3 a V_{1}^{* 2}+c\right) & \alpha & 0 \\
1 & -1 & 1 \\
0 & -\beta & -\gamma
\end{array}\right] .
\end{aligned}
$$

\subsection{Local passivity}

Let us apply the results in Sec. 7.2 to find the local passive parameter range for $\mathrm{CNNs}$ made of cubic
Chua's circuits. Defining

$$
\begin{aligned}
A_{4} \triangleq & 4 \alpha\left(3 a V_{1}^{* 2}+c\right)-(\alpha+1)^{2} \\
A_{2} \triangleq & 8 \alpha\left(3 a V_{1}^{* 2}+c\right) \gamma^{2}+4 \gamma \alpha\left(3 a V_{1}^{* 2}+c\right) \beta \\
& -2(1+\alpha)^{2} \gamma^{2} \\
A_{0} \triangleq & 4 \alpha\left(3 a V_{1}^{* 2}+c\right) \gamma^{4}+4 \gamma^{3} \alpha\left(3 a V_{1}^{* 2}+c\right) \beta \\
& -\gamma^{4}(1+\alpha)^{2}
\end{aligned}
$$

and simplifying we obtain

$$
\begin{aligned}
A_{4}= & 4 \alpha\left(3 a V_{1}^{* 2}+c\right)-(1+\alpha)^{2} \\
A_{2}= & 4 \alpha \gamma\left(3 a V_{1}^{* 2}+c\right)(2 \gamma+\beta) \\
& -2(1+\alpha)^{2} \gamma^{2} \\
A_{0}= & 4 \gamma^{3} \alpha\left(3 a V_{1}^{* 2}+c\right)(\gamma+\beta) \\
& -\gamma^{4}(1+\alpha)^{2} .
\end{aligned}
$$

The local passive regions are given by

$$
\begin{aligned}
& \alpha\left(3 a V_{1}^{* 2}+c\right)+1 \geq 0 \quad \mathbb{A N D} \quad \beta \gamma \geq-\left[\alpha\left(3 a V_{1}^{* 2}+c\right)+1\right] \gamma^{2} \quad \mathbb{A N D} \\
& \left\{\left\{A_{4}>0 \quad \mathbb{A N D} \quad\left[\left(-\frac{A_{2}^{2}}{4 A_{4}}+A_{0} \geq 0 \quad \mathbb{A N D} \quad A_{2} \leq 0\right) \quad \mathbb{O R} \quad\left(\begin{array}{llll}
A_{0} \geq 0 & \mathbb{A N D} & A_{2}>0
\end{array}\right)\right]\right\} \quad \mathbb{O R}\right. \\
& \left.\left[A_{4}=0 \quad \mathbb{A N D} \quad A_{2} \geq 0 \quad \mathbb{A N D} \quad A_{0} \geq 0\right]\right\} \quad \mathbb{A N D} \quad \alpha\left(3 a V_{1}^{* 2}+c\right) \geq 0 \quad \mathbb{A N D} \quad 0 \leq \alpha\left(3 a V_{1}^{* 2}+c\right) \gamma^{2}
\end{aligned}
$$

Simplifying Eq. (231) we obtain

$$
\begin{aligned}
& \beta \gamma \geq-\left[\alpha\left(3 a V_{1}^{* 2}+c\right)+1\right] \gamma^{2} \quad \mathbb{A N D} \\
& \left\{\left\{A_{4}>0 \quad \mathbb{A N D} \quad\left[\left(-\frac{A_{2}^{2}}{4 A_{4}}+A_{0} \geq 0 \quad \mathbb{A N D} \quad A_{2} \leq 0\right) \quad \mathbb{O R} \quad\left(\begin{array}{llll}
A_{0} \geq 0 & \mathbb{A N D} & A_{2}>0
\end{array}\right)\right]\right\} \quad \mathbb{O R}\right. \\
& \left.\left[A_{4}=0 \quad \mathbb{A N D} \quad A_{2} \geq 0 \quad \mathbb{A N D} \quad A_{0} \geq 0\right]\right\} \quad \mathbb{A N D} \quad \alpha\left(3 a V_{1}^{* 2}+c\right) \geq 0
\end{aligned}
$$

from which we have

$$
\begin{gathered}
\beta \gamma \geq-\left[\alpha\left(3 a V_{1}^{* 2}+c\right)+1\right] \gamma^{2} \quad \mathbb{A N D} \quad\left\{\left\{\left[4 \alpha\left(3 a V_{1}^{* 2}+c\right)-(\alpha+1)^{2}\right]>0 \quad \mathbb{A N D}\right.\right. \\
{\left[\left(-\frac{\left[4 \alpha \gamma\left(3 a V_{1}^{* 2}+c\right)(2 \gamma+\beta)-2(1+\alpha)^{2} \gamma^{2}\right]^{2}}{4\left[4 \alpha\left(3 a V_{1}^{* 2}+c\right)-(\alpha+1)^{2}\right]}+\left[4 \gamma^{3} \alpha\left(3 a V_{1}^{* 2}+c\right)(\gamma+\beta)-\gamma^{4}(1+\alpha)^{2}\right] \geq 0 \quad \mathbb{A N D}\right.\right.} \\
\left.\left[4 \alpha \gamma\left(3 a V_{1}^{* 2}+c\right)(2 \gamma+\beta)-2(1+\alpha)^{2} \gamma^{2}\right] \leq 0\right) \quad \mathbb{O} \mathbb{R} \quad\left(\left[4 \gamma^{3} \alpha\left(3 a V_{1}^{* 2}+c\right)(\gamma+\beta)-\gamma^{4}(1+\alpha)^{2}\right] \geq 0 \quad \mathbb{A N D}\right.
\end{gathered}
$$




$$
\begin{gathered}
\left.\left.\left.\left[4 \alpha \gamma\left(3 a V_{1}^{* 2}+c\right)(2 \gamma+\beta)-2(1+\alpha)^{2} \gamma^{2}\right]>0\right)\right]\right\} \quad \mathbb{O} \quad\left[\left[4 \alpha\left(3 a V_{1}^{* 2}+c\right)-(\alpha+1)^{2}\right]=0 \quad \mathbb{A N D}\right. \\
\left.\left.\left[4 \alpha \gamma\left(3 a V_{1}^{* 2}+c\right)(2 \gamma+\beta)-2(1+\alpha)^{2} \gamma^{2}\right] \geq 0 \quad \mathbb{A N D} \quad\left[4 \gamma^{3} \alpha\left(3 a V_{1}^{* 2}+c\right)(\gamma+\beta)-\gamma^{4}(1+\alpha)^{2}\right] \geq 0\right]\right\} \\
\mathbb{A N D} \quad \alpha\left(3 a V_{1}^{* 2}+c\right) \geq 0 .
\end{gathered}
$$

In general it would be very difficult to find an explicit expression for the local passive parameter regions defined in Eq. (233). Much insights can be gained, however, by assuming $\gamma=0$, which greatly simplifies the algebra.

\subsubsection{Local passivity regions when $\gamma=0$}

When $\gamma=0$ we have

$$
\begin{aligned}
& A_{4}=4 \alpha\left(3 a v_{1}^{* 2}+c\right)-(1+\alpha)^{2} \\
& A_{2}=0, A_{0}=0 .
\end{aligned}
$$

In this special case, the local passive parameter region is given by

$$
A_{4} \geq 0 \quad \mathbb{A N D} \quad \alpha\left(3 a V_{1}^{* 2}+c\right) \geq 0
$$

from which we have

$$
\begin{gathered}
4 \alpha\left(3 a V_{1}^{* 2}+c\right)-(1+\alpha)^{2} \geq 0 \quad \mathbb{A N D} \\
\alpha\left(3 a V_{1}^{* 2}+c\right) \geq 0 .
\end{gathered}
$$

Since the second inequality is redundant, the local passive parameter region is simply given by

$$
4 \alpha\left(3 a V_{1}^{* 2}+c\right)-(1+\alpha)^{2} \geq 0 .
$$

Observe that the local passive regions of the Chua's circuit cell depends on the position of the equilibrium points. Therefore, in order to pin down the exact local passive regions, we must specify the explicit expressions of $V_{1}^{*}$. Defining

$$
\mathfrak{D}=\frac{I^{* 2}}{4}+\frac{c^{3}}{27 a^{3}}
$$

we can derive the following analytical expressions for the local passive parameter regions in Chua's circuit.
Case 1. $\mathfrak{D}>0$.

In this case we have only one equilibrium point

$$
\begin{aligned}
V_{1}^{*}= & \left(\frac{I^{*}}{2}+\sqrt{\frac{I^{* 2}}{4}+\frac{c^{3}}{27 a^{3}}}\right)^{1 / 3} \\
& +\left(\frac{I^{*}}{2}-\sqrt{\frac{I^{* 2}}{4}+\frac{c^{3}}{27 a^{3}}}\right)^{1 / 3} .
\end{aligned}
$$

It follows from Eqs. (237) and (238) that the local passive parameter region is given by

$$
\begin{aligned}
\mathfrak{D}= & \frac{I^{* 2}}{4}+\frac{c^{3}}{27 a^{3}}>0, \\
& 4 \alpha\left\{3 a \left[\left(\frac{I^{*}}{2}+\sqrt{\frac{I^{* 2}}{4}+\frac{c^{3}}{27 a^{3}}}\right)^{1 / 3}\right.\right. \\
& \left.\left.+\left(\frac{I^{*}}{2}-\sqrt{\frac{I^{* 2}}{4}+\frac{c^{3}}{27 a^{3}}}\right)^{1 / 3}\right]^{2}+c\right\} \\
& -(1+\alpha)^{2} \geq 0 .
\end{aligned}
$$

Case 2. $\mathfrak{D}=0$

In this case we have two equilibrium points at

$$
V_{1 a}^{*}=2\left(\frac{I^{*}}{2}\right)^{1 / 3}, V_{1 b}^{*}=\left(\frac{I^{*}}{2}\right)^{1 / 3} .
$$

It follows from Eqs. (237) and (238) that the local passive parameter region is given by

$$
\begin{aligned}
\mathfrak{D}= & \frac{I^{* 2}}{4}+\frac{c^{3}}{27 a^{3}}=0, \\
& 4 \alpha\left\{3 a\left[2\left(\frac{I^{*}}{2}\right)^{1 / 3}\right]^{2}+c\right\}-(1+\alpha)^{2} \geq 0, \\
& 4 \alpha\left\{3 a\left[\left(\frac{I^{*}}{2}\right)^{1 / 3}\right]^{2}+c\right\}-(1+\alpha)^{2} \geq 0 .
\end{aligned}
$$


Remark. The region given in Eq. (242) usually consists of intersection of curves. Therefore, it usually consists of isolated points.

Case 3. $\mathfrak{D}<0$

In this case we have three equilibrium points at

$$
\begin{aligned}
& V_{1 a}^{*}=2 \sqrt{\frac{|\Lambda|}{3}} \cos \frac{\psi}{3}, \\
& V_{1 b}^{*}=-2 \sqrt{\frac{|\Lambda|}{3}} \cos (\psi / 3-\pi / 3), \\
& V_{1 c}^{*}=-2 \sqrt{\frac{|\Lambda|}{3}} \cos (\psi / 3+\pi / 3)
\end{aligned}
$$

where

$$
\Lambda=-\frac{c}{a} \text {. }
$$

The local passive parameter region in this case is given by:

$$
\begin{aligned}
\mathfrak{D}= & \frac{I^{* 2}}{4}+\frac{c^{3}}{27 a^{3}}<0, \\
& 4 \alpha\left\{3 a\left[2 \sqrt{\frac{|\Lambda|}{3}} \cos \frac{\psi}{3}\right]^{2}+c\right\}-(1+\alpha)^{2} \geq 0 \\
& 4 \alpha\left\{3 a\left[-2 \sqrt{\frac{|\Lambda|}{3}} \cos (\psi / 3-\pi / 3)\right]^{2}+c\right\} \\
& -(1+\alpha)^{2} \geq 0, \\
& 4 \alpha\left\{3 a\left[-2 \sqrt{\frac{|\Lambda|}{3}} \cos (\psi / 3+\pi / 3)\right]^{2}+c\right\} \\
& -(1+\alpha)^{2} \geq 0, \\
\psi= & \cos ^{-1}\left(\frac{I^{*} / 2}{\sqrt{|\Lambda|^{3} / 27}}\right) .
\end{aligned}
$$

Remark. Since the Chua's circuit is a realistic model of a physical circuit, we can expect that if there are three equilibrium points, then at least one of them will be locally active. This observation can be exploited to simplify the task of finding the local passive regions.

Once the equilibrium points are found, the boundaries of the local passive regions can be easily computed by a simple program as illustrated by examples in the next section.

\subsection{Edge-of-chaos regions when $\gamma=0$}

Once the local passive region is found, the local active region is simply its set complement. By definition, the edge-of-chaos region is a subset of the local active regions where all poles of the cell impedance matrix $\mathbf{Z}_{Q}(s)$ are in the left-half $s$-plane. Thus, to find the edge-of-chaos region, we must find the locations of the poles of $\mathbf{Z}_{Q}(s)$, which are the roots of the polynomial

$$
\begin{aligned}
P(s) \triangleq & s^{4}-\left[a_{11}+a_{22}+2 a_{33}\right] s^{3}+\left[a_{11} a_{33}-a_{13} a_{31}\right. \\
& +a_{22} a_{33}-a_{23} a_{32}+\left(a_{11}+a_{33}\right)\left(a_{22}+a_{33}\right) \\
& \left.-a_{12} a_{21}\right] s^{2}-\left[\left(a_{11}+a_{33}\right)\left(a_{22} a_{33}-a_{23} a_{32}\right)\right. \\
& +\left(a_{22}+a_{33}\right)\left(a_{11} a_{33}-a_{13} a_{31}\right)+a_{12}\left(-a_{21} a_{33}\right. \\
& \left.\left.+a_{23} a_{31}\right)+a_{21}\left(-a_{12} a_{33}+a_{13} a_{32}\right)\right] s \\
& +\left[\left(a_{11} a_{33}-a_{13} a_{31}\right)\left(a_{22} a_{33}-a_{23} a_{32}\right)\right. \\
& \left.-\left(-a_{12} a_{33}+a_{13} a_{32}\right)\left(-a_{21} a_{33}+a_{23} a_{31}\right)\right] . \\
= & s^{4}+p_{3} s^{3}+p_{2} s^{2}+p_{1} s+p_{0}
\end{aligned}
$$

where

$$
\begin{aligned}
p_{3} \triangleq & -\left[a_{11}+a_{22}+2 a_{33}\right] \\
p_{2} \triangleq & a_{11} a_{33}-a_{13} a_{31}+a_{22} a_{33}-a_{23} a_{32} \\
& +\left(a_{11}+a_{33}\right)\left(a_{22}+a_{33}\right)-a_{12} a_{21} \\
p_{1} \triangleq & -\left[\left(a_{11}+a_{33}\right)\left(a_{22} a_{33}-a_{23} a_{32}\right)\right. \\
& +\left(a_{22}+a_{33}\right)\left(a_{11} a_{33}-a_{13} a_{31}\right) \\
& +a_{12}\left(-a_{21} a_{33}+a_{23} a_{31}\right) \\
& \left.+a_{21}\left(-a_{12} a_{33}+a_{13} a_{32}\right)\right] \\
p_{0} \triangleq & \left(a_{11} a_{33}-a_{13} a_{31}\right)\left(a_{22} a_{33}-a_{23} a_{32}\right) \\
& -\left(-a_{12} a_{33}+a_{13} a_{32}\right)\left(-a_{21} a_{33}+a_{23} a_{31}\right) .
\end{aligned}
$$

Substituting the parameters in Eq. (228) into Eq. (246) and letting $\gamma=0$ we have

$$
\begin{aligned}
& p_{3}=-\left(a_{11}+a_{22}\right) \\
& p_{2}=-a_{23} a_{32}+a_{11} a_{22}-a_{12} a_{21} \\
& p_{1}=a_{11} a_{23} a_{32} \\
& p_{0}=0 .
\end{aligned}
$$


Combining Eqs. (247) and (245), we obtain

$$
P(s)=s^{4}-\left(a_{11}+a_{22}\right) s^{3}-\left(a_{23} a_{32}-a_{11} a_{22}+a_{12} a_{21}\right) s^{2}+a_{11} a_{23} a_{32} s .
$$

From Eq. (176) we obtain

$$
\mathbf{Z}_{Q}(s)=\frac{1}{s^{3}-\left(a_{11}+a_{22}\right) s^{2}-\left(a_{23} a_{32}-a_{11} a_{22}+a_{12} a_{21}\right) s+a_{11} a_{23} a_{32}}\left[\begin{array}{cc}
Z_{11}(s) & Z_{12}(s) \\
Z_{21}(s) & Z_{22}(s)
\end{array}\right]
$$

where

$$
\begin{aligned}
& Z_{11}(s)=s^{2}-a_{22} s-a_{23} a_{32}, \\
& Z_{12}(s)=a_{12} s, \\
& Z_{21}(s)=a_{21} s, \\
& Z_{22}(s)=s^{2}-a_{11} s .
\end{aligned}
$$

It follows from Eqs. (228) and (249) that the poles are given by the roots of

$$
s^{3}+\left[\alpha\left(3 a v_{1}^{* 2}+c\right)+1\right] s^{2}-\left[-\beta-\alpha\left(3 a v_{1}^{* 2}+c\right)+\alpha\right] s+\alpha\left(3 a v_{1}^{* 2}+c\right) \beta=0 .
$$

Defining $B \triangleq 3 a v_{1}^{* 2}$ we can rewrite Eq. (251) as follows

$$
s^{3}+[\alpha(B+c)+1] s^{2}+[\beta+\alpha(B+c-1)] s+\alpha \beta(B+c)=0 .
$$

Let us consider the case $\mathfrak{D}>0$, where we have only one equilibrium point:

$$
V_{1}^{*}=\left(\frac{I^{*}}{2}+\sqrt{\frac{I^{* 2}}{4}+\frac{c^{3}}{27 a^{3}}}\right)^{1 / 3}+\left(\frac{I^{*}}{2}-\sqrt{\frac{I^{* 2}}{4}+\frac{c^{3}}{27 a^{3}}}\right)^{1 / 3} .
$$

From Eqs. (240) and (252) we obtain the following edge-of-chaos parameter region:

$$
\begin{aligned}
\mathfrak{D}= & \frac{I^{* 2}}{4}+\frac{c^{3}}{27 a^{3}}>0, \quad 4 \alpha\left\{3 a\left[\left(\frac{I^{*}}{2}+\sqrt{\frac{I^{* 2}}{4}+\frac{c^{3}}{27 a^{3}}}\right)^{1 / 3}+\left(\frac{I^{*}}{2}-\sqrt{\frac{I^{* 2}}{4}+\frac{c^{3}}{27 a^{3}}}\right)^{1 / 3}\right]^{2}+c\right\} \\
& -(1+\alpha)^{2} \geq 0, \quad \text { all roots of } s^{3}+[\alpha(B+c)+1] s^{2}+[\beta+\alpha(B+c-1)] s+\alpha \beta(B+c)=0 \\
& \text { are in the left-half } s \text {-plane }
\end{aligned}
$$

where

$$
B=3 a\left[\left(\frac{I^{*}}{2}+\sqrt{\frac{I^{* 2}}{4}+\frac{c^{3}}{27 a^{3}}}\right)^{1 / 3}+\left(\frac{I^{*}}{2}-\sqrt{\frac{I^{* 2}}{4}+\frac{c^{3}}{27 a^{3}}}\right)^{1 / 3}\right]^{2}
$$

Remark. It suffices to consider only the case $\mathfrak{D}>0$ because when $\mathfrak{D}<0$, there will be at least one pole on the right-half $s$-plane. The case $\mathfrak{D}=0$ corresponds to the boundary of the edge-of-chaos region because there is at least one pole on the imaginary axis of the $s$-plane.

\section{Examples}

We now present some examples of CNNs made of Chua's circuit cells. The state equation of an $N \times N$ cubic Chua's circuit CNN (with two diffusion coefficients $D_{1}$ and $D_{2}$ ) is given by 


$$
\begin{aligned}
\dot{V}_{1}(i, j)= & \alpha\left[V_{2}(i, j)-a V_{1}^{3}(i, j)-c V_{1}(i, j)\right]+D_{1}\left[V_{1}(i-1, j)+V_{1}(i, j-1)+V_{1}(i, j+1)+V_{1}(i+1, j)\right. \\
& \left.-4 V_{1}(i, j)\right] \\
\dot{V}_{2}(i, j)= & V_{1}(i, j)-V_{2}(i, j)+V_{3}(i, j)+D_{2}\left[V_{2}(i-1, j)+V_{2}(i, j-1)+V_{2}(i, j+1)+V_{2}(i+1, j)\right. \\
& \left.-4 V_{2}(i, j)\right] \\
\dot{V}_{3}(i, j)= & -\beta V_{2}(i, j)-\gamma V_{3}(i, j), \quad 1 \leq i \leq N, \quad 1 \leq i \leq N
\end{aligned}
$$

In all simulations to be presented in this section, the initial conditions of the CNNs are given by pseudorandom variables uniformly distributed over the interval $(-1,1)$. A toroidal boundary condition is used for all examples.

\subsection{Case 1: $c / a>0$}

In this case we know that the first condition in Eq. (240) is always satisfied for any $I^{*} \in(-\infty, \infty)$. Hence, the local passive parameter regions for case $\mathfrak{D}>0$ are solely determined by the second condition

$$
\begin{gathered}
4 \alpha\left\{3 a \left[\left(\frac{I^{*}}{2}+\sqrt{\frac{I^{* 2}}{4}+\frac{c^{3}}{27 a^{3}}}\right)^{1 / 3}\right.\right. \\
\left.\left.+\left(\frac{I^{*}}{2}-\sqrt{\frac{I^{* 2}}{4}+\frac{c^{3}}{27 a^{3}}}\right)^{1 / 3}\right]^{2}+c\right\} \\
-(1+\alpha)^{2} \geq 0
\end{gathered}
$$

10.1.1. $\quad a=1$ and $c=0.143$

Figure 17 shows the $I^{*}-\alpha$ parameter plane for the local active region and the restricted local passive region. In this case there are no (unrestricted) local passive parameter regions. This means that for a CNN in this category, any homogeneous pattern can be destabilized by choosing large coupling current biases. One easy way to generate large coupling current biases is to make the coupling coefficients sufficiently large and to choose a nonhomogeneous initial condition. There is no edge-of-chaos region in this case.

Our simulation results show that for this kind of CNN, whenever $\alpha \leq 0$ only unstable patterns are observed. Some chaotic patterns are observed when we choose $\alpha>0$.

Figure 18 shows our simulation results with pa- rameters $\alpha=4, \beta=14, \gamma=0, a=1, c=0.143$, $D_{1}=0.5$ and $D_{2}=0.5$. The size of CNN is $60 \times 60$.

\subsection{Case 2: $c / a \leq 0$}

In this case, the first condition in Eq. (240) reduces to

$$
I^{*}>\sqrt{-\frac{4 c^{3}}{27 a^{2}}} \quad \mathbb{O R} \quad I^{*}<-\sqrt{-\frac{4 c^{3}}{27 a^{2}}} .
$$

The second condition in Eq. (240) remains unchanged.

Consider next the case when $a=-1$ and $c=2$. Figure 19 shows the $I^{*}-\alpha$ parameter plane for the local active, restricted passive and edge-of-chaos regions. In this case we again see that there are no (unrestricted) local passive parameter regions. This means that for any CNN in this category, any homogeneous pattern can be destabilized by choosing large coupling current biases. There are two outstanding features in the behaviors of this $\mathrm{CNN}$ :

1. When $\alpha<0$ and $I^{*}$ is small enough, the CNN cell is locally active independent of $\alpha$. This means that when $\alpha<0$ the trivial homogeneous pattern is unstable.

2. When $\alpha>0$, the CNN cell is restricted locally passive if $I^{*}$ is small enough. This means that when $\alpha>0$ the trivial homogeneous pattern is stable. However, as $I^{*}$ increases, it will eventually become unstable. This theoretical prediction had been verified by carrying out the following two simulation experiments:

(a) Fix $\alpha$ and initial condition, and with small coupling strength $D_{1}$ and $D_{2}$, we observe the CNN settles down to a trivial homogeneous pattern. However, upon increasing $D_{1}$ and $D_{2}$ beyond some critical value, we find the CNN becomes unstable. 


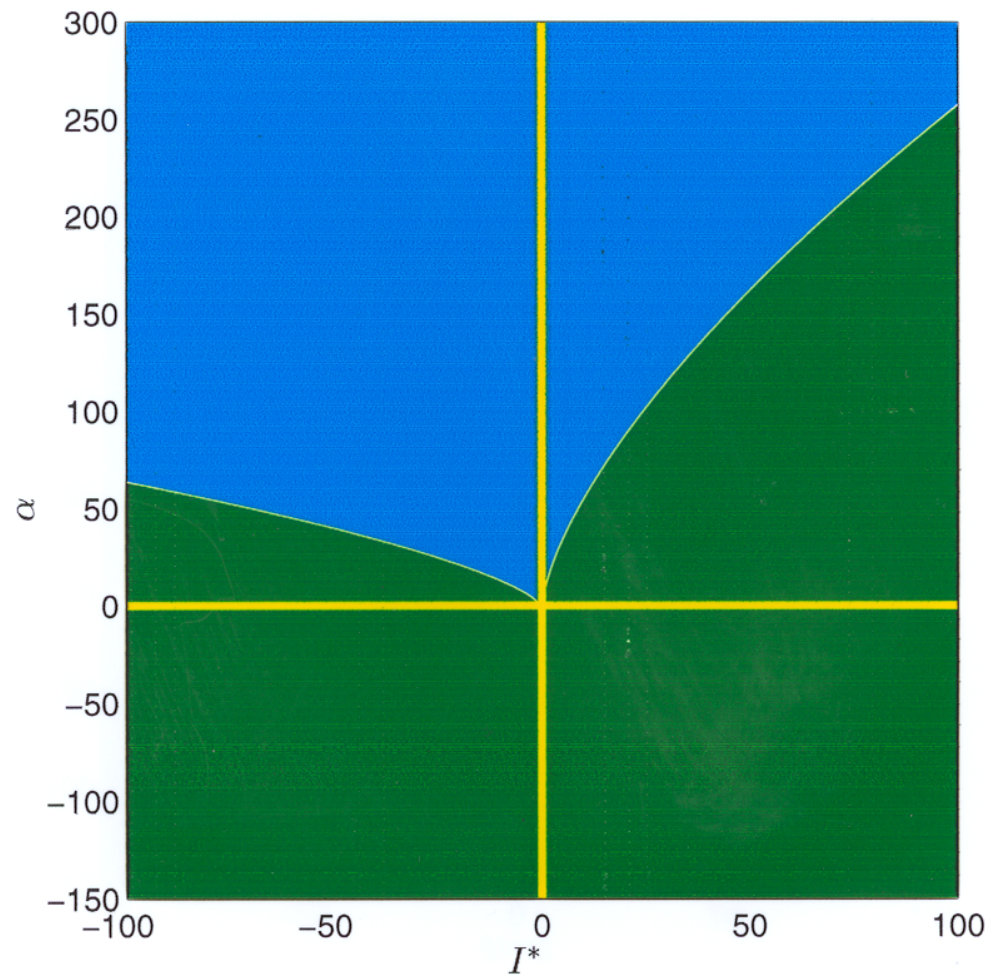

Fig. 17. Parameter space in the $I^{*}-\alpha$ plane. The fixed parameters are given by: $\gamma=0, a=1$ and $c=0.143$. The local active regions and restricted local passive regions are colored in green and cyan, respectively.

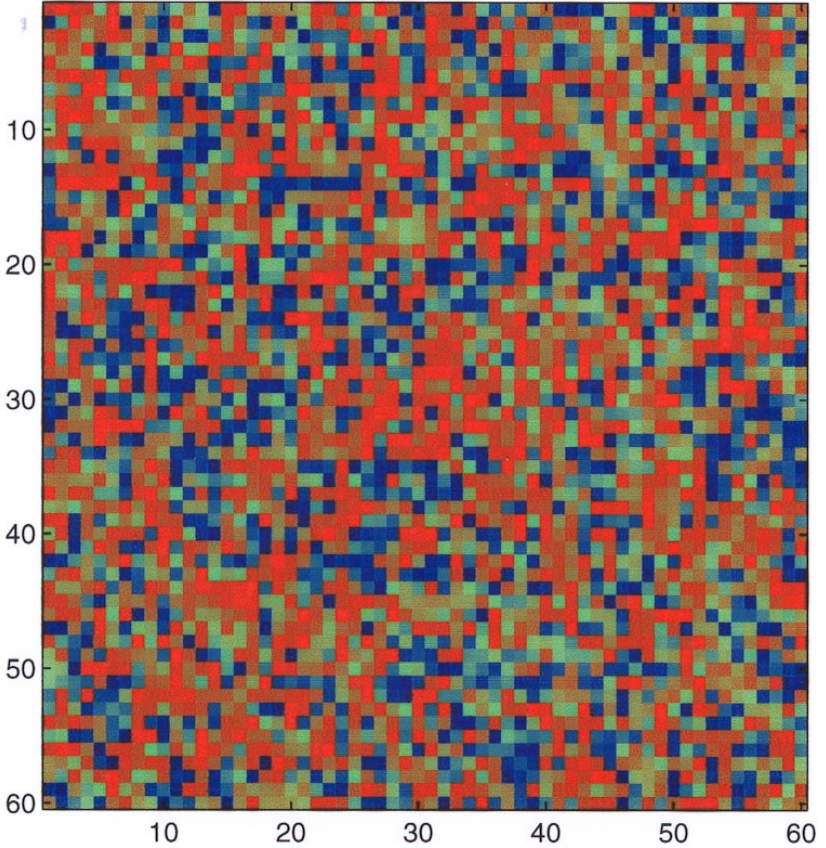

(a)

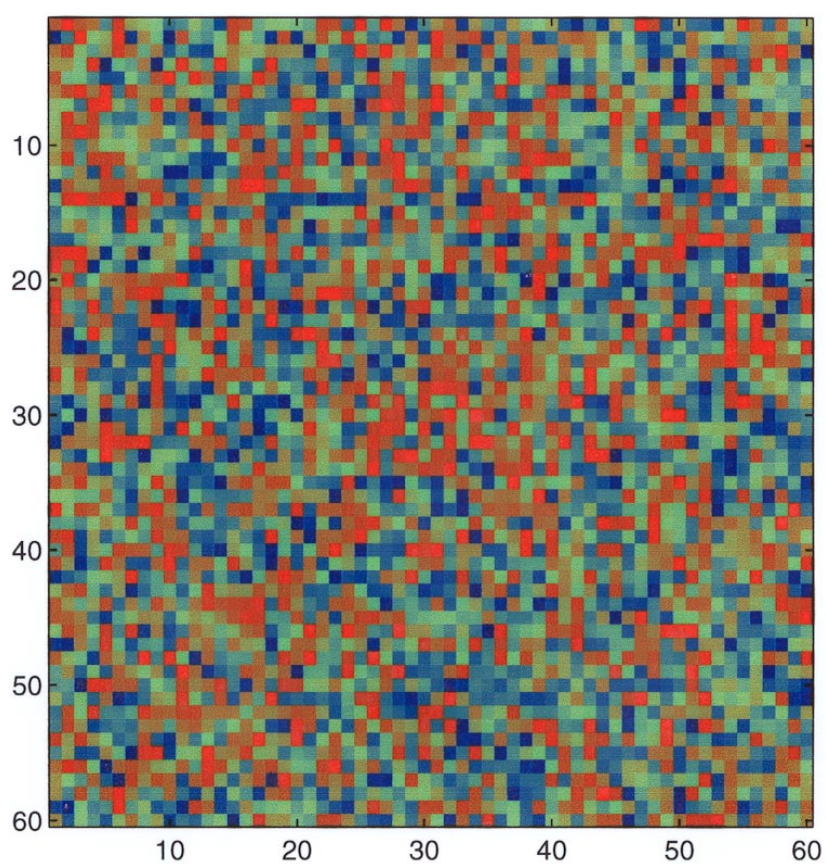

(b)

Fig. 18. Simulation results of Chua's circuit CNN with parameters: $\alpha=4, \beta=14, \gamma=0, a=1, c=0.143, D_{1}=0.5$ and $D_{2}=0.5$. (a) $V_{1}(10)$. (b) $V_{2}(10)$. (c) $V_{1}(20)$. (d) $V_{2}(20)$. (e) $V_{1}(50)$. (f) $V_{2}(50)$. (g) $V_{1}(100)$. (h) $V_{2}(100)$. (i) $V_{1}(200)$. (j) $V_{2}(200)$. (k) $V_{1}(400)$. (l) $V_{2}(400)$. (m) $V_{1}(450)$. (n) $V_{2}(450)$. (o) $V_{1}(500)$. (p) $V_{2}(500)$. (q) $V_{1}(750)$. (r) $V_{2}(750)$. (s) $V_{1}(900)$. (t) $V_{2}(900)$. (u) $V_{1}(1000)$. (v) $V_{2}(1000)$. (w) $V_{1}(1350)$. (x) $V_{2}(1350)$. (y) $V_{1}(1500)$. (z) $V_{2}(1500)$. (aa) $V_{1}(1800)$. (ab) $V_{2}(1800)$. (ac) $V_{1}(2250)$. (ad) $V_{2}(2250)$. (ae) $V_{1}(2700)$. (af) $V_{2}(2700)$. 


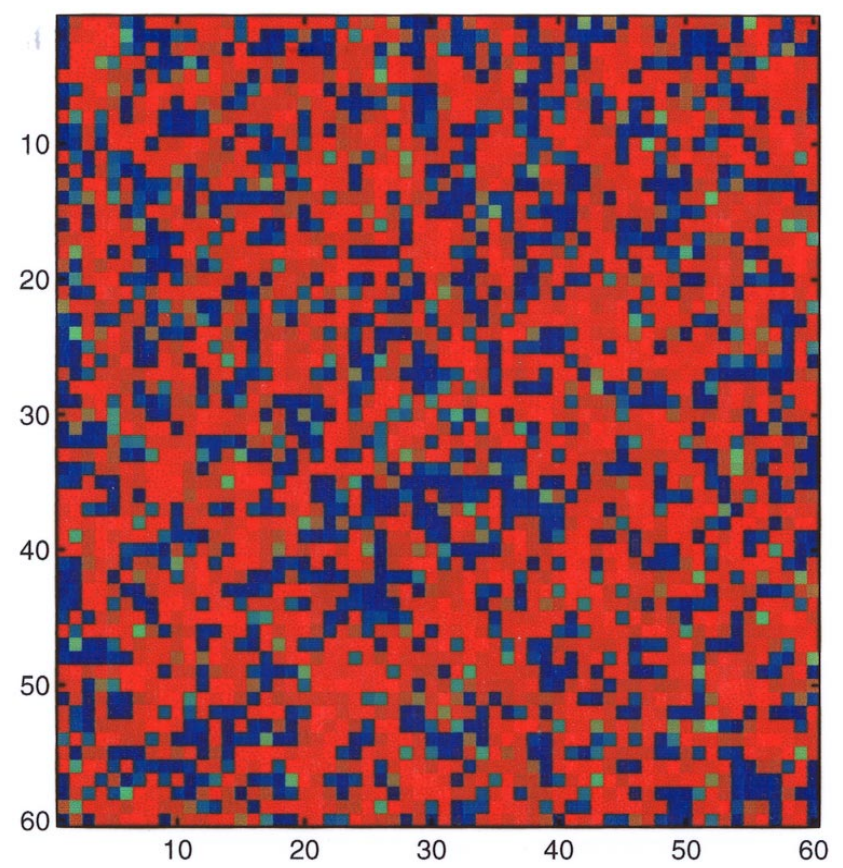

(c)

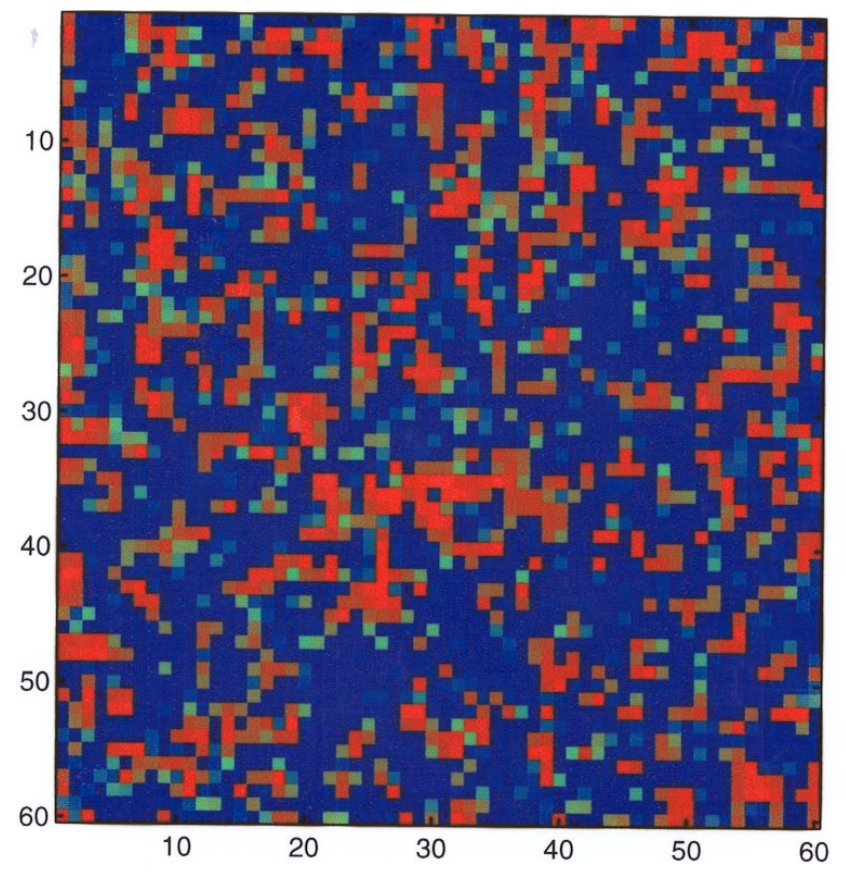

(e)

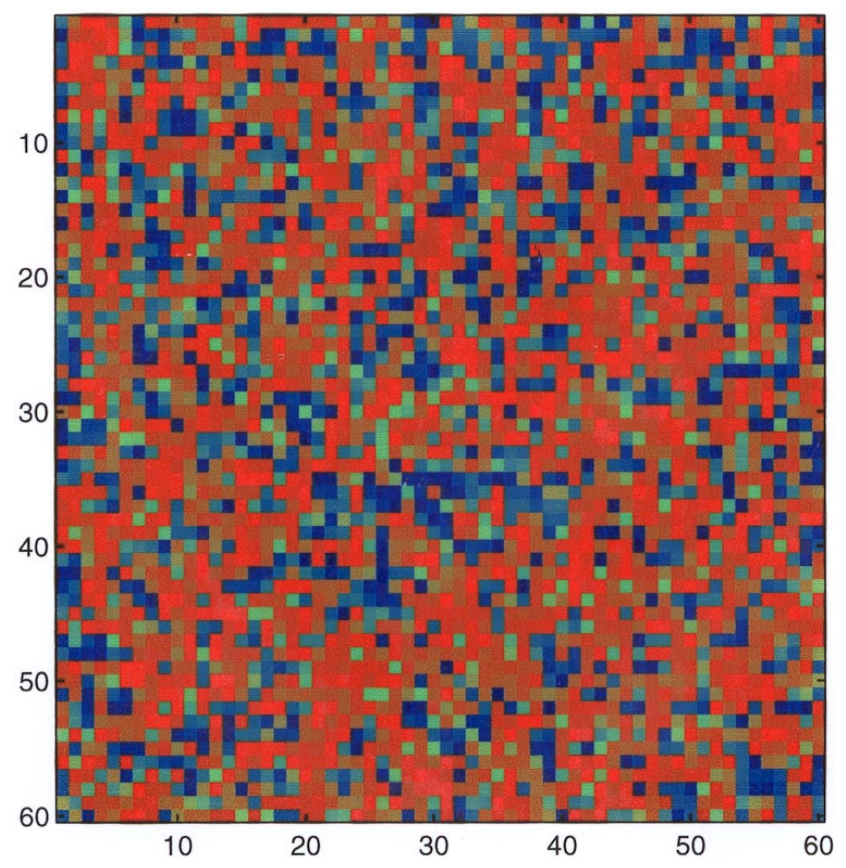

(d)

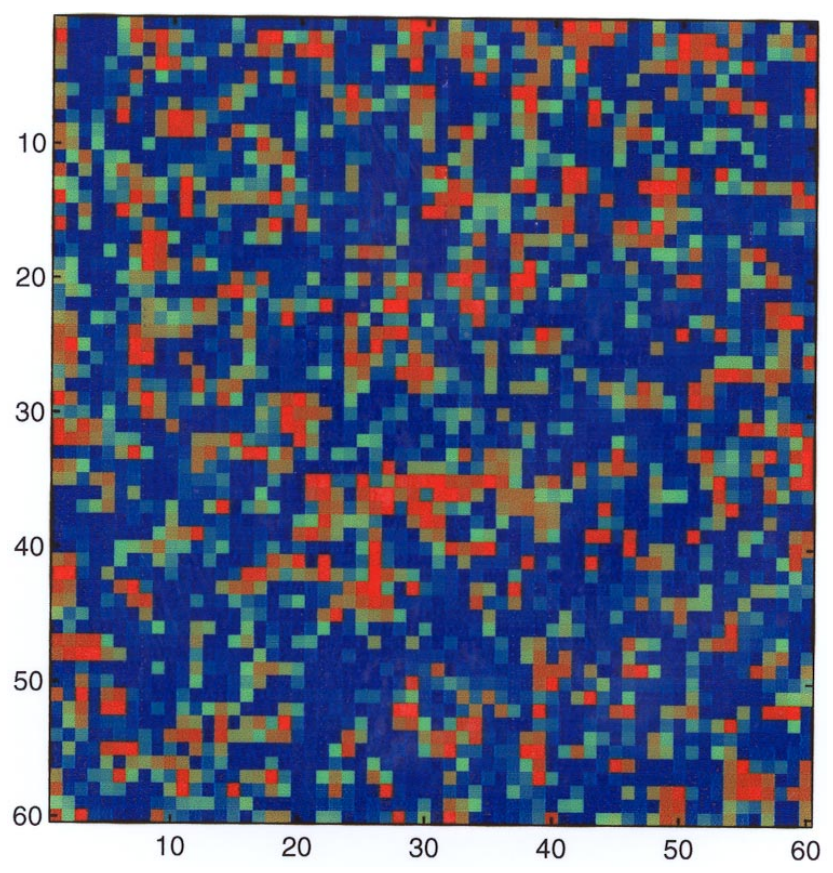

(f)

Fig. 18. (Continued) 


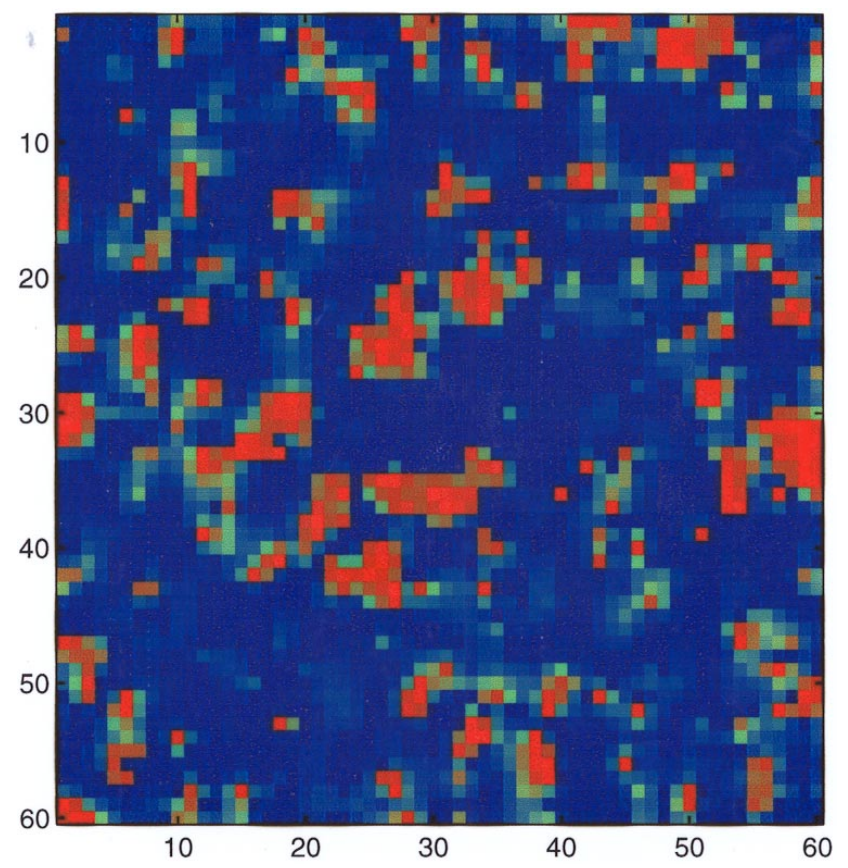

(g)

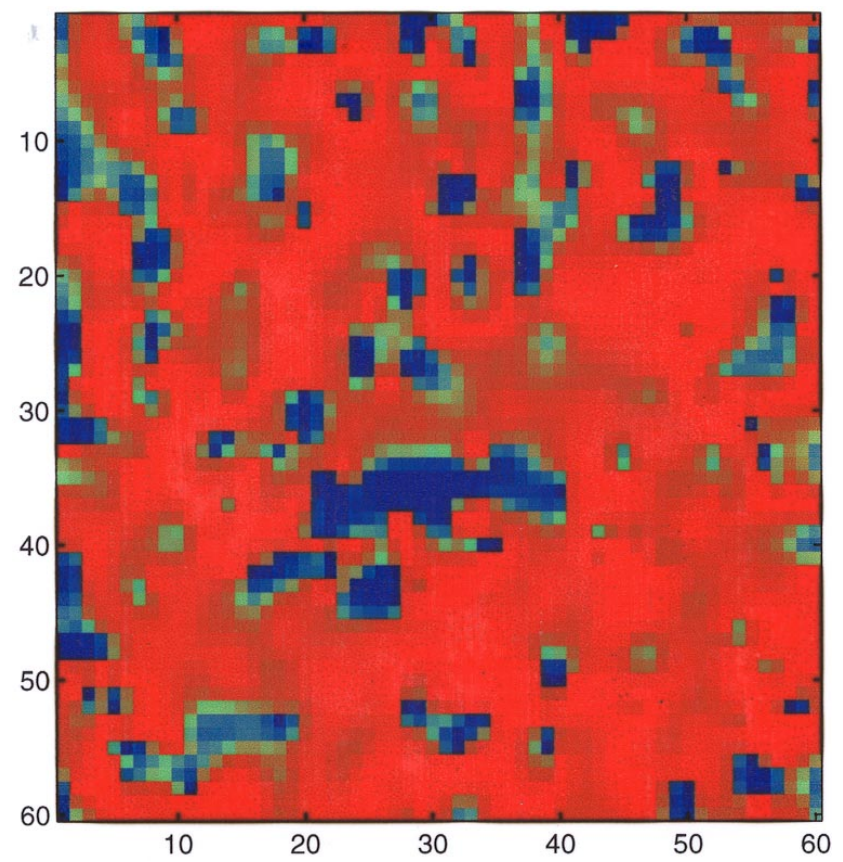

(i)

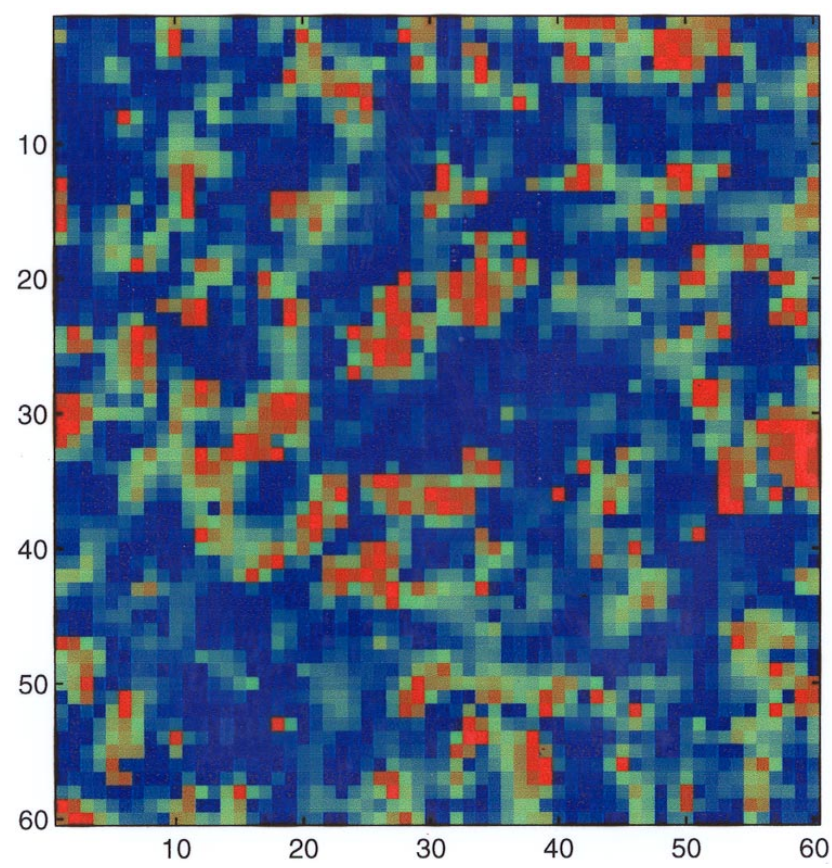

(h)

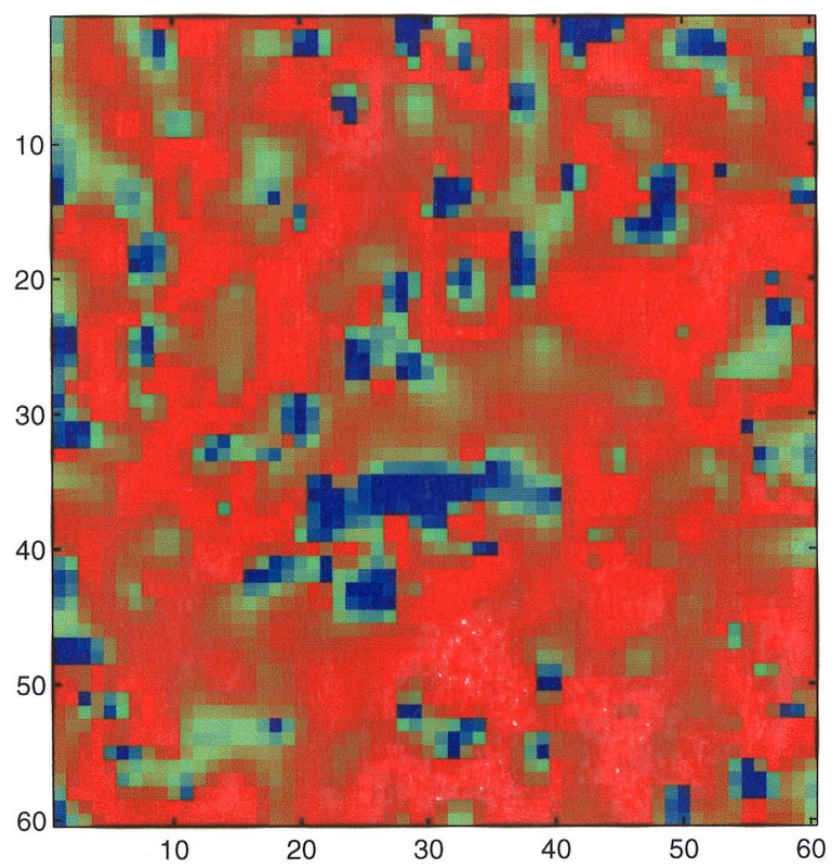

(j)

Fig. 18. (Continued) 


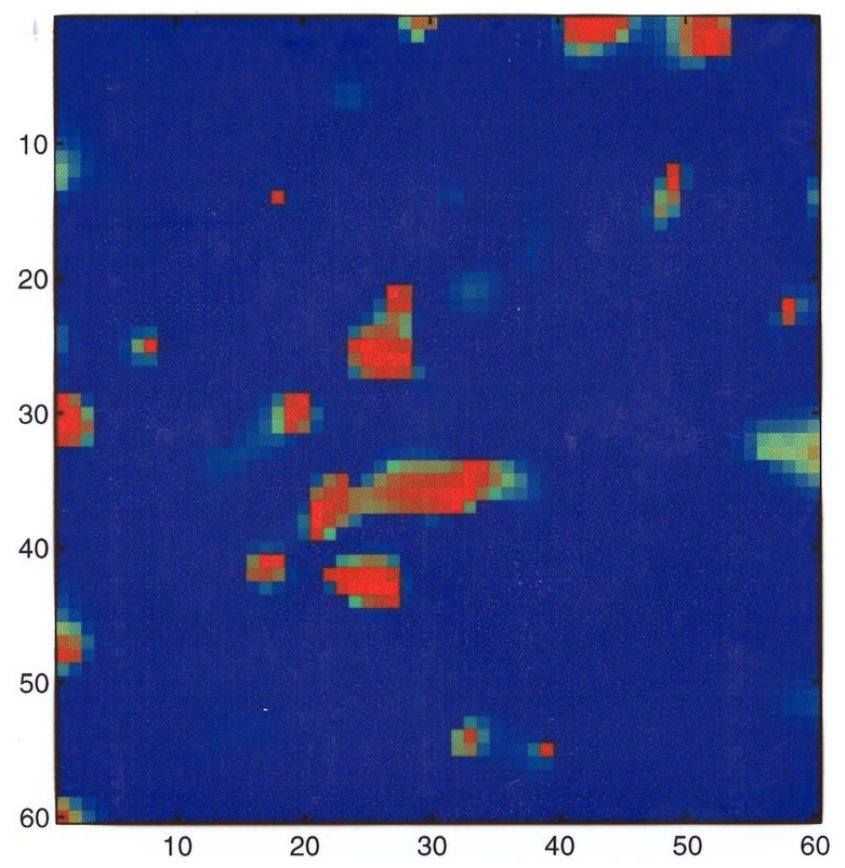

(k)

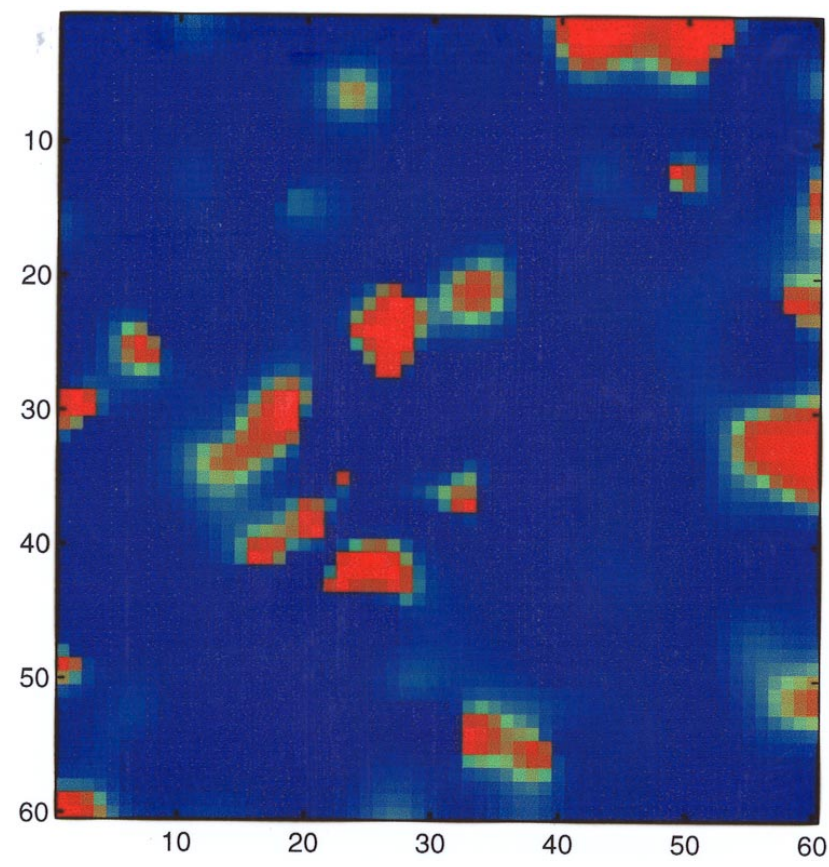

$(\mathrm{m})$

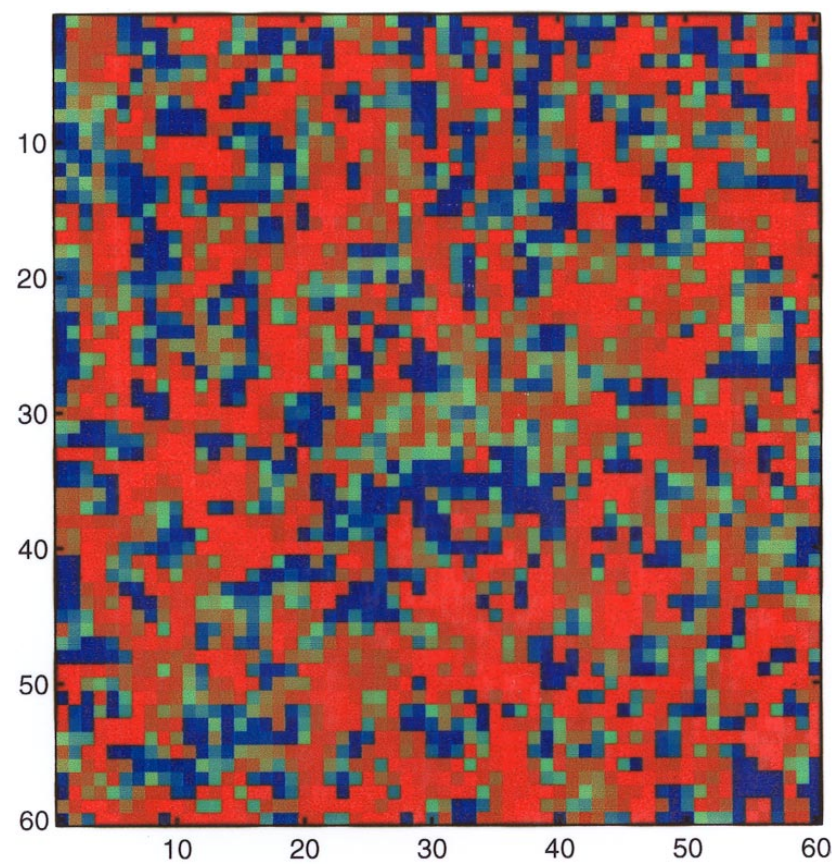

(l)

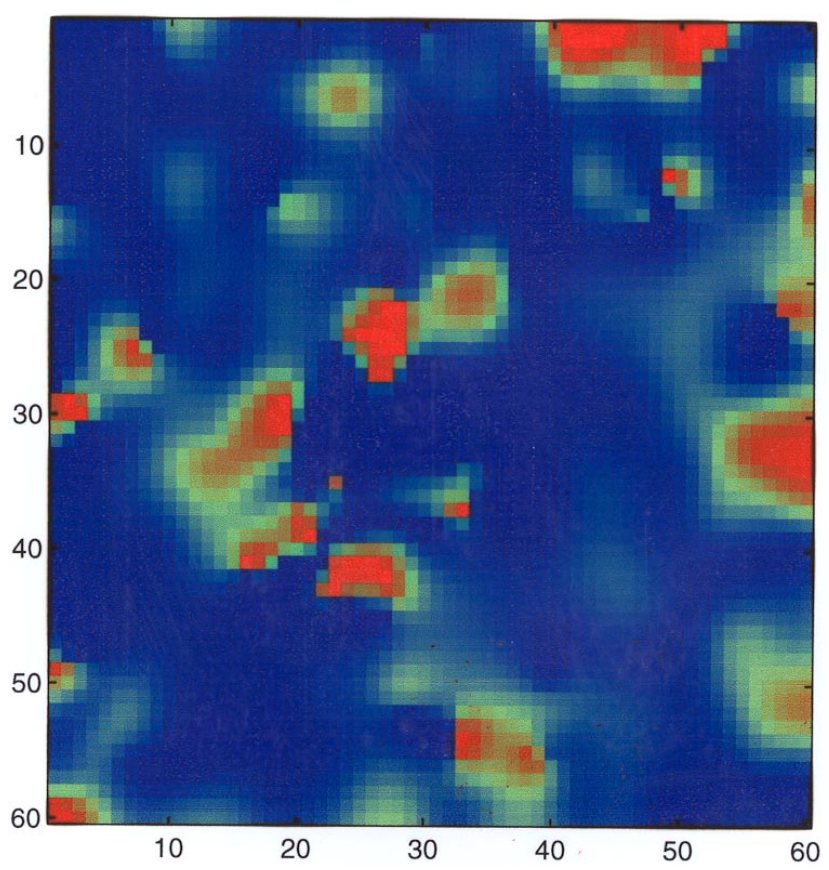

(n)

Fig. 18. (Continued) 


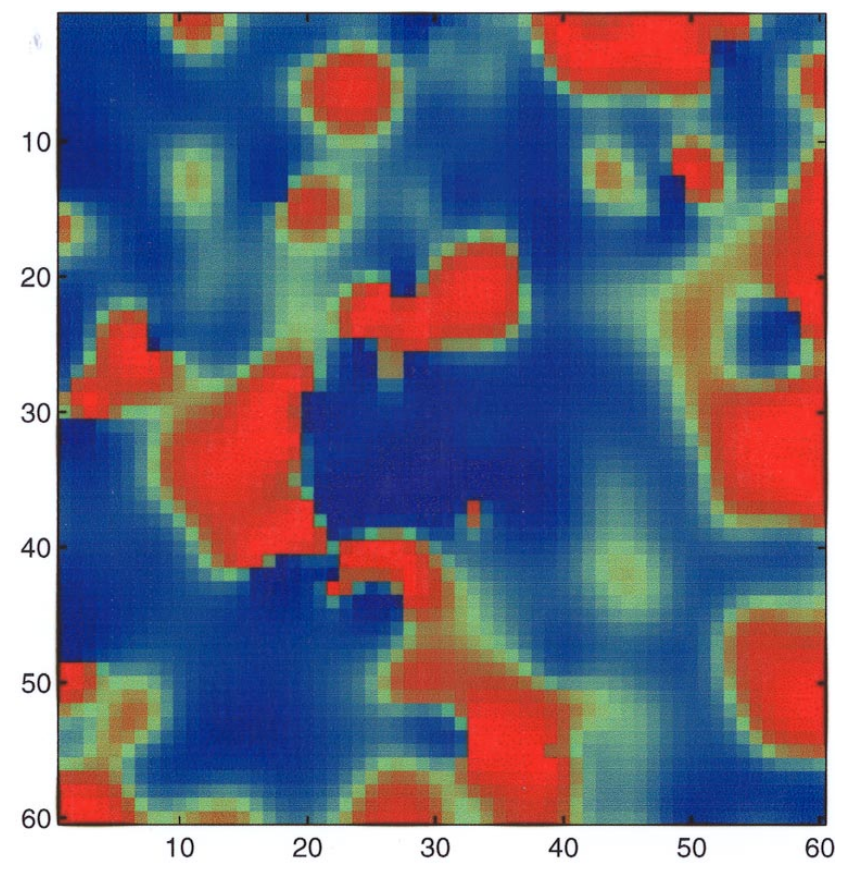

(o)

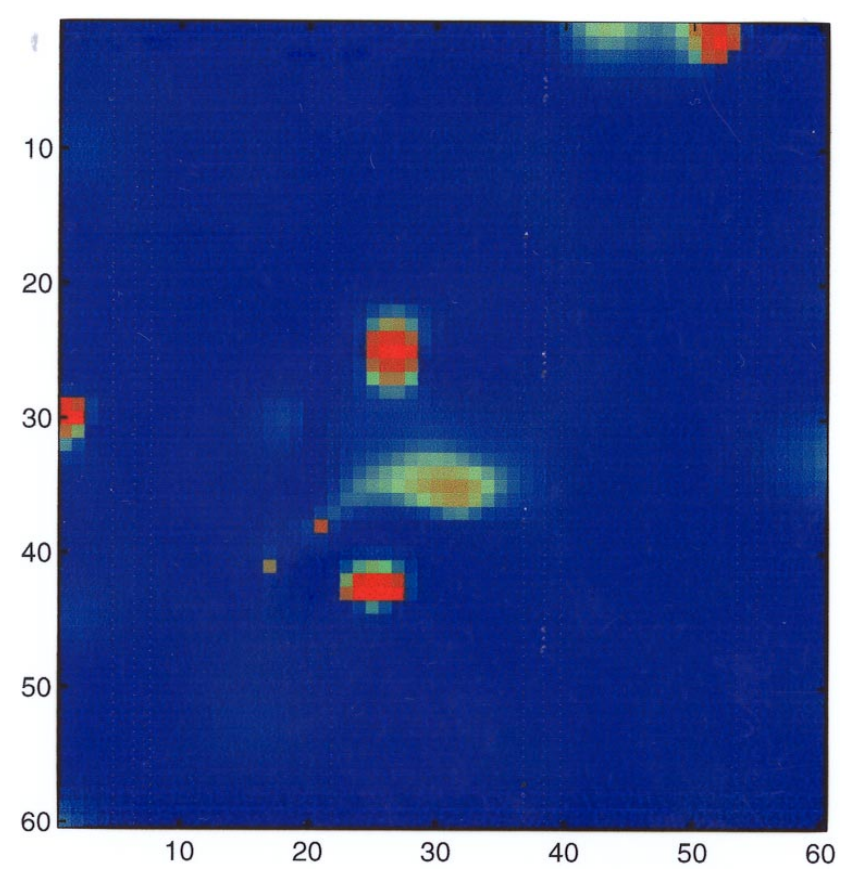

(q)

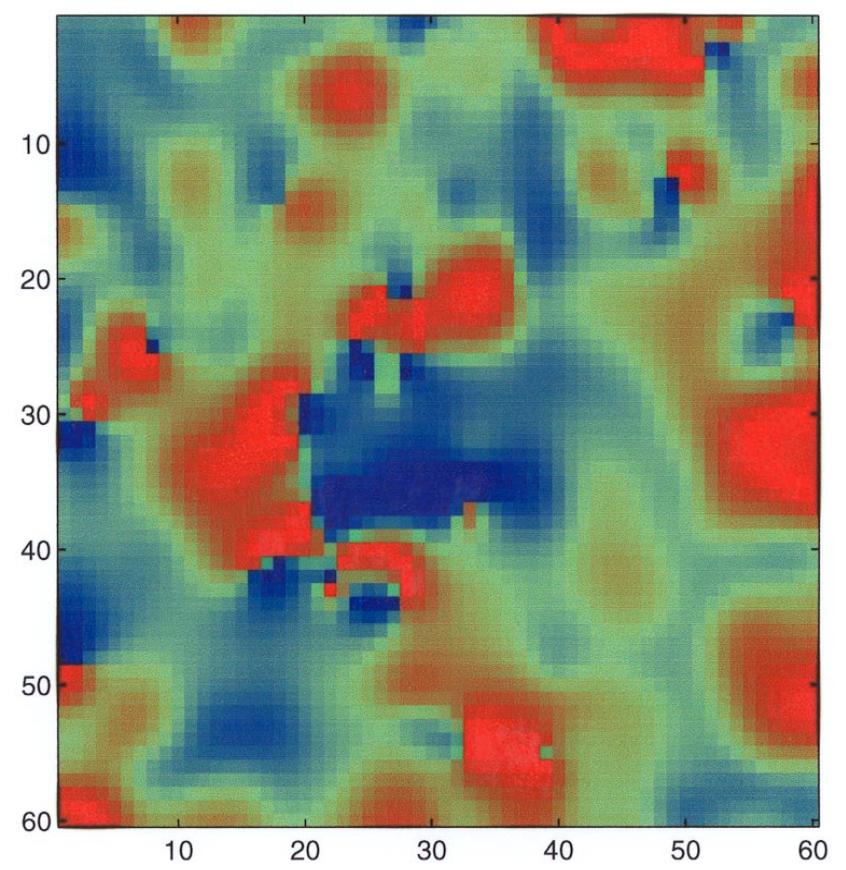

(p)

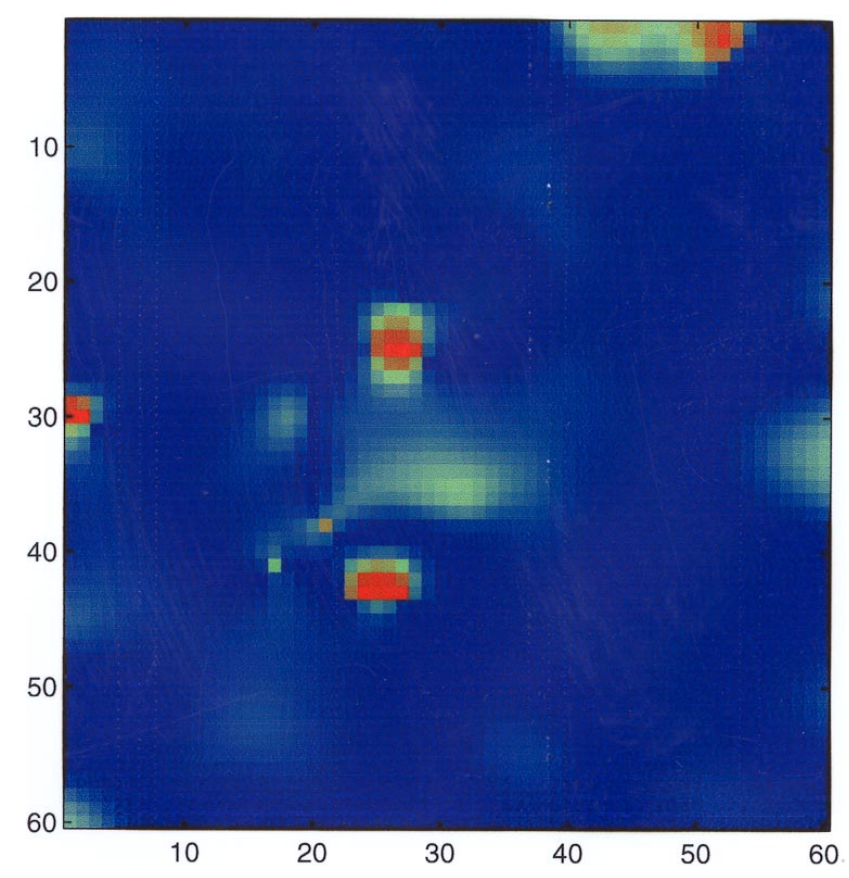

(r)

Fig. 18. (Continued) 


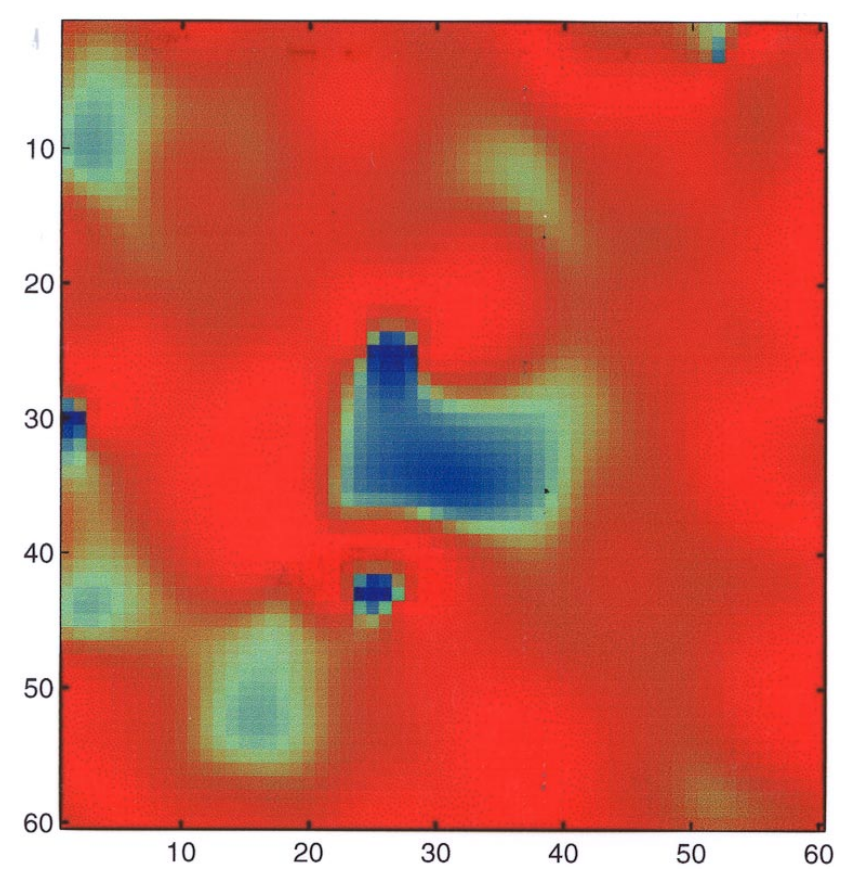

(s)

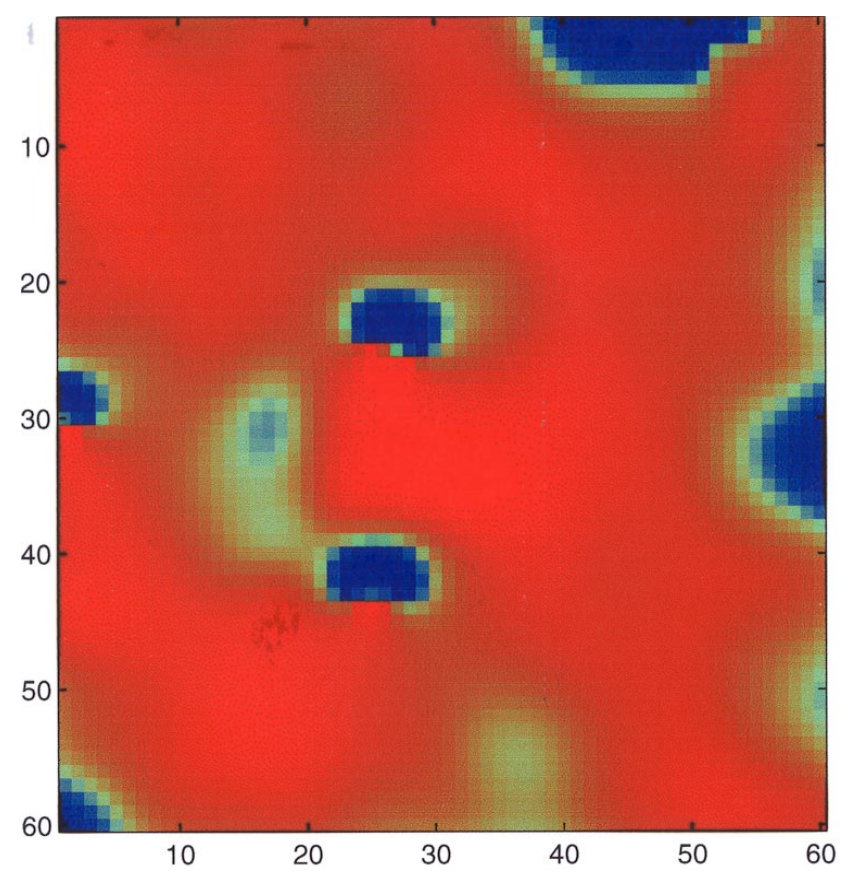

(u)

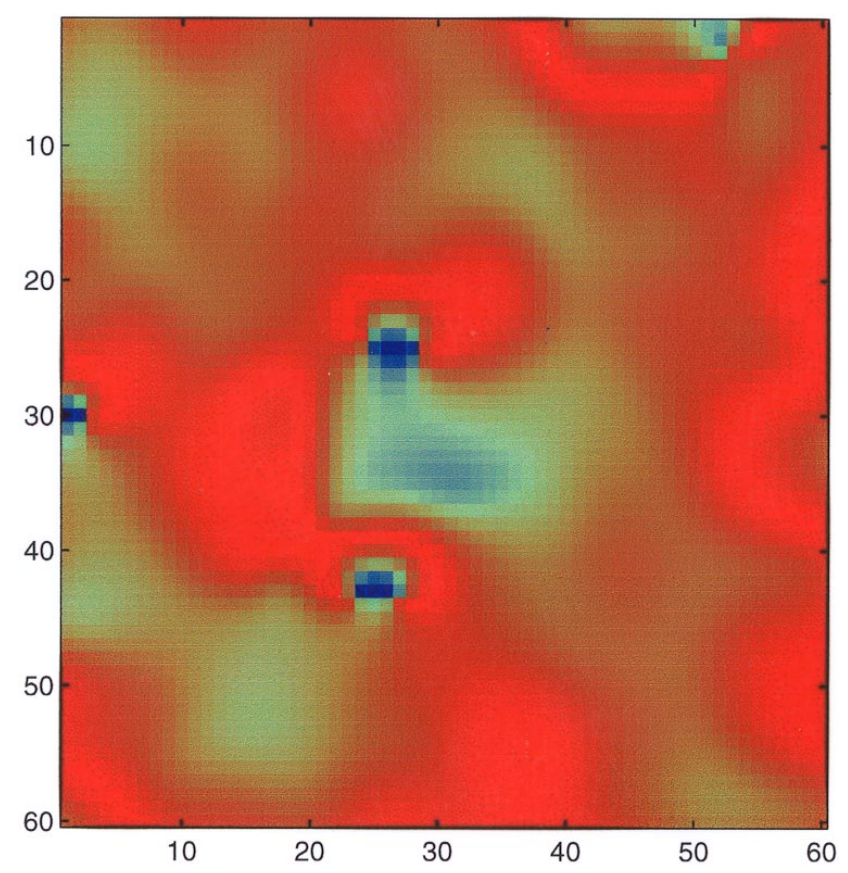

(t)

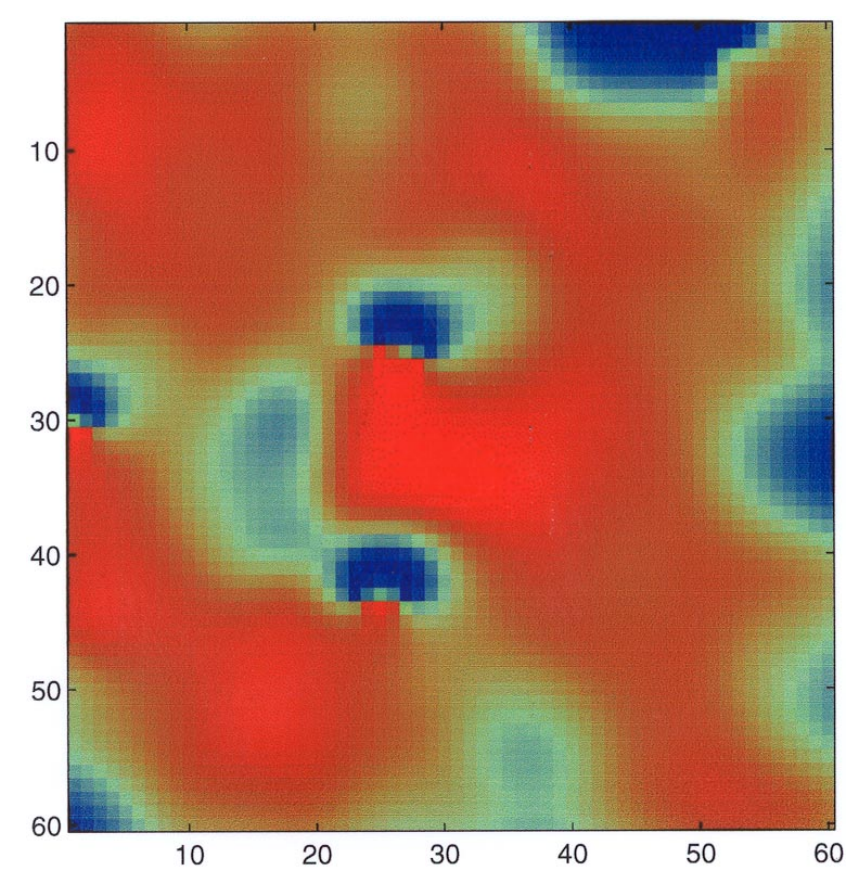

(v)

Fig. 18. (Continued) 


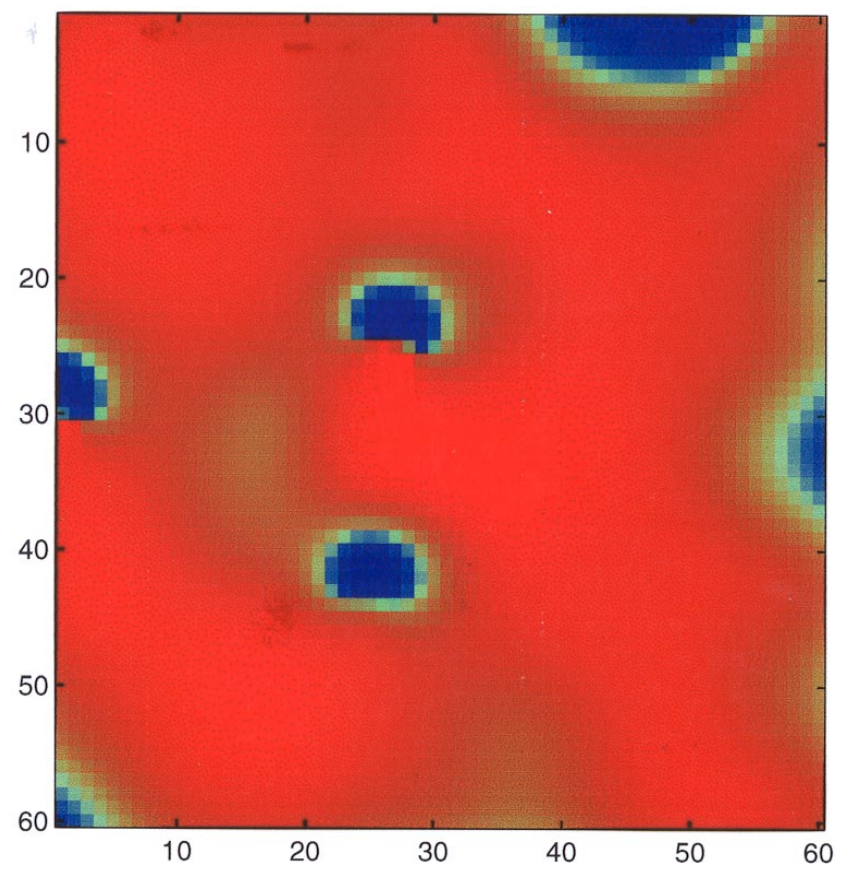

(w)

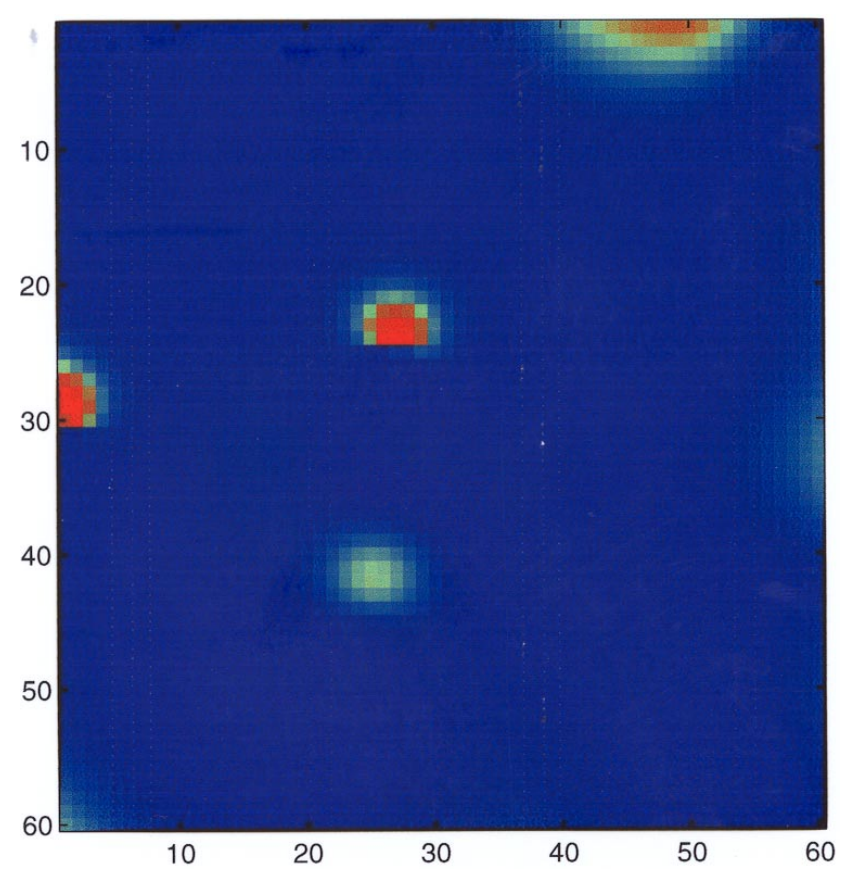

(y)

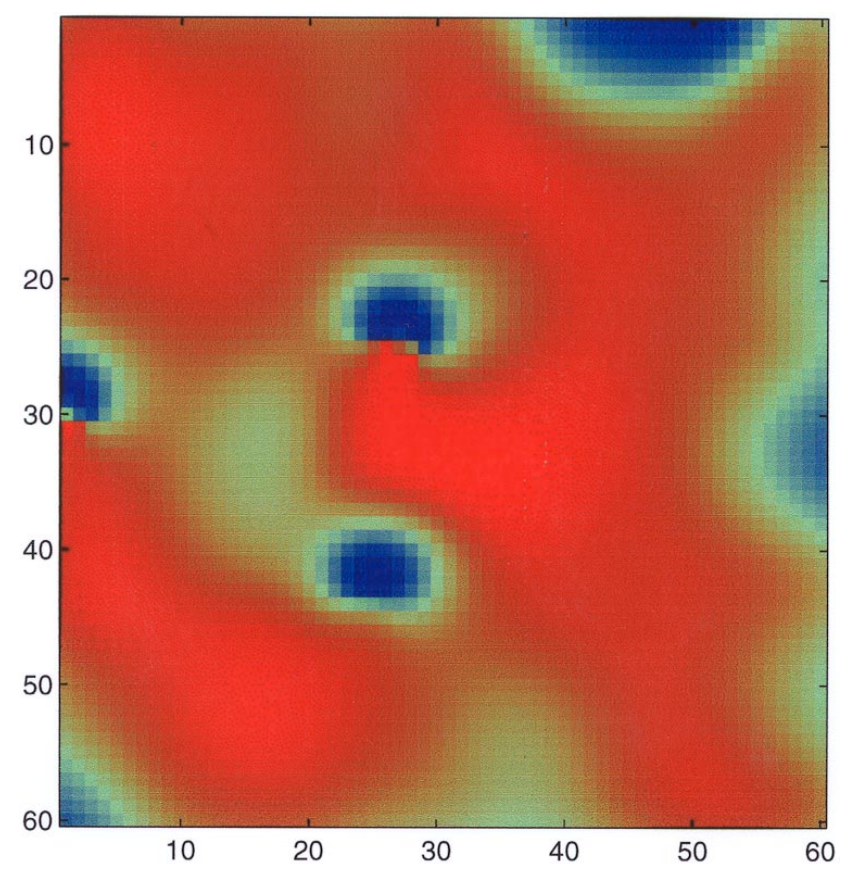

(x)

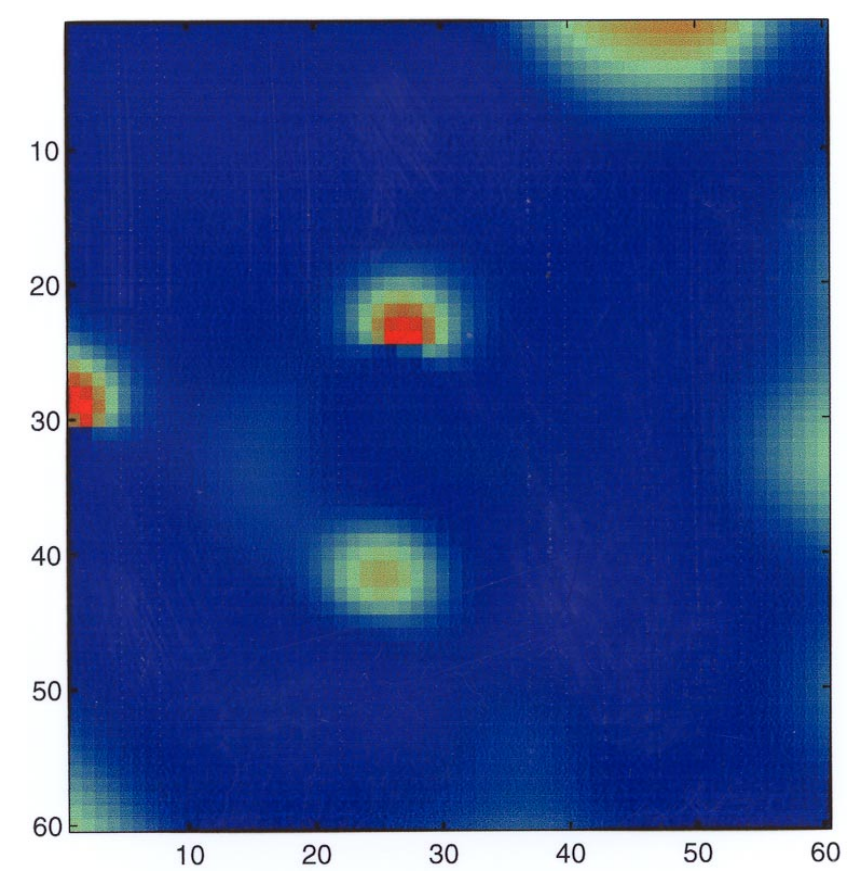

(z)

Fig. 18. (Continued) 


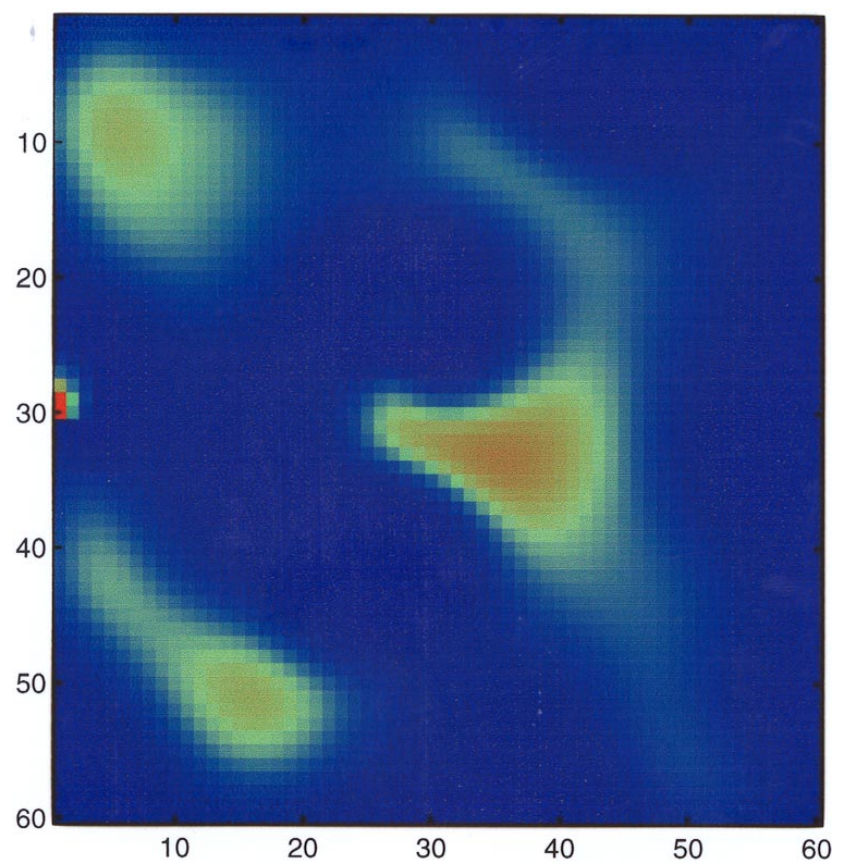

(aa)

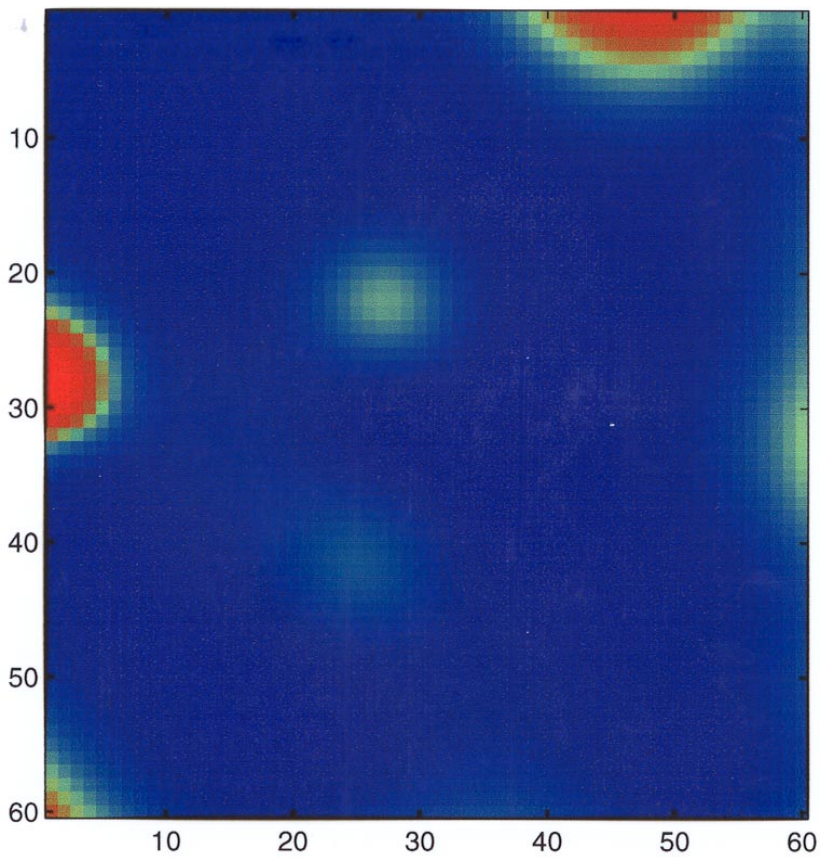

(ac)

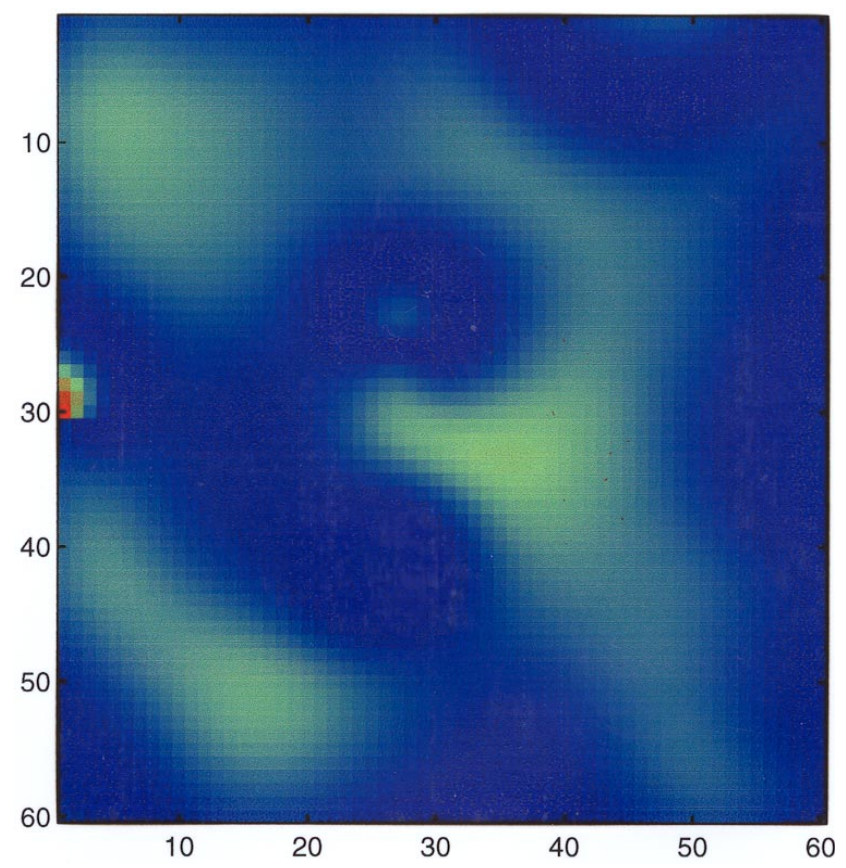

(ab)

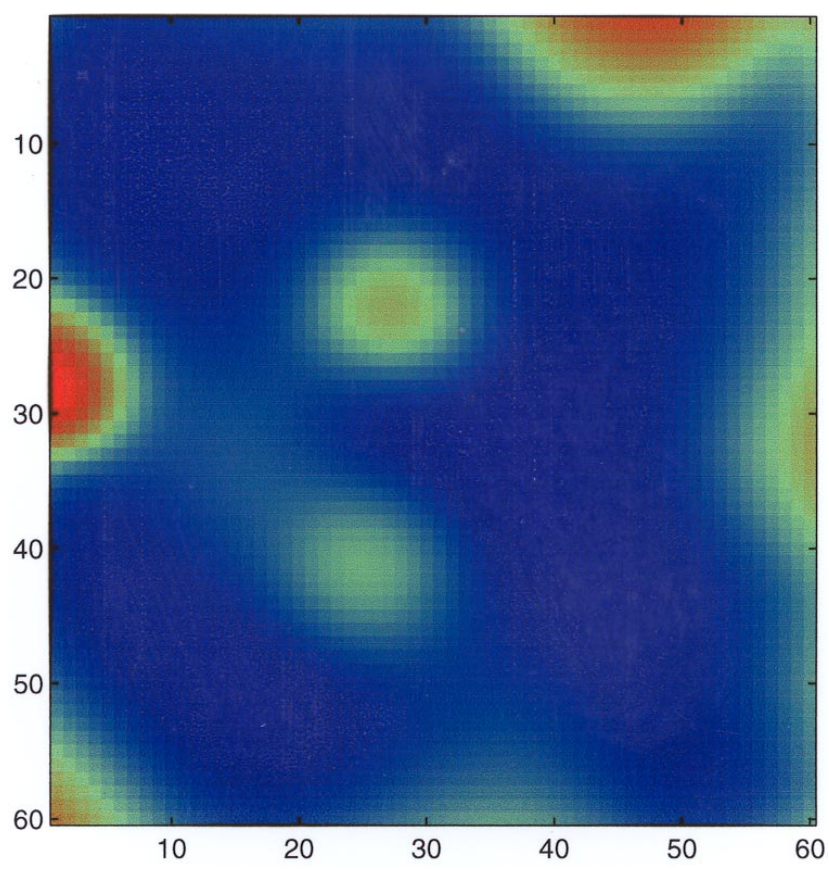

(ad)

Fig. 18. (Continued) 


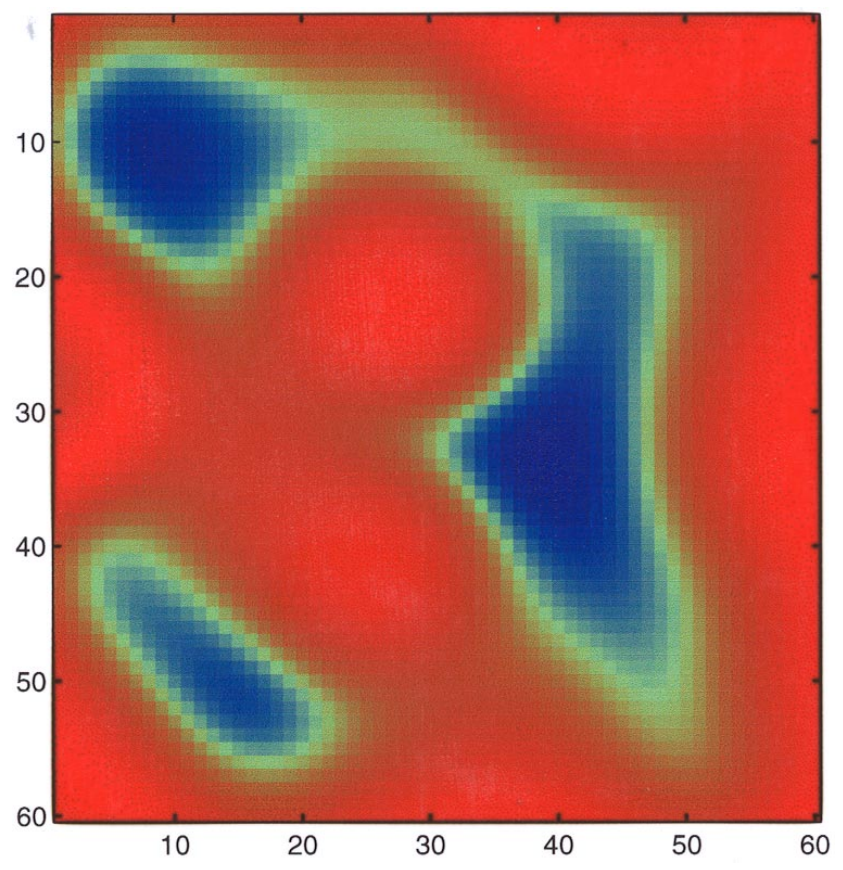

(ae)

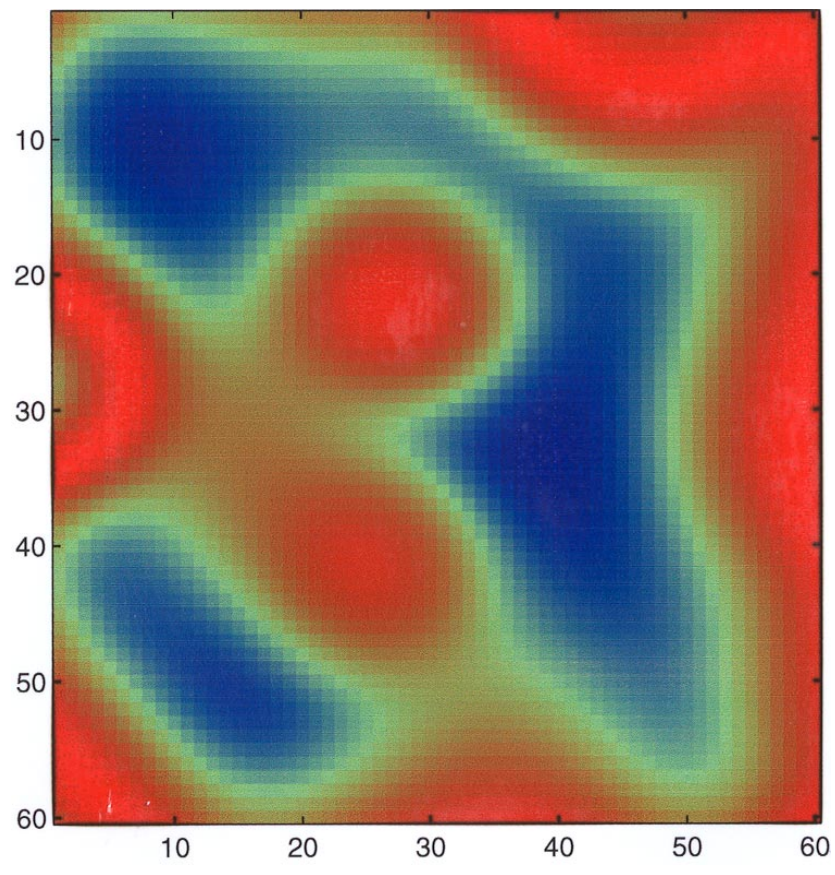

(af)

Fig. 18. (Continued)

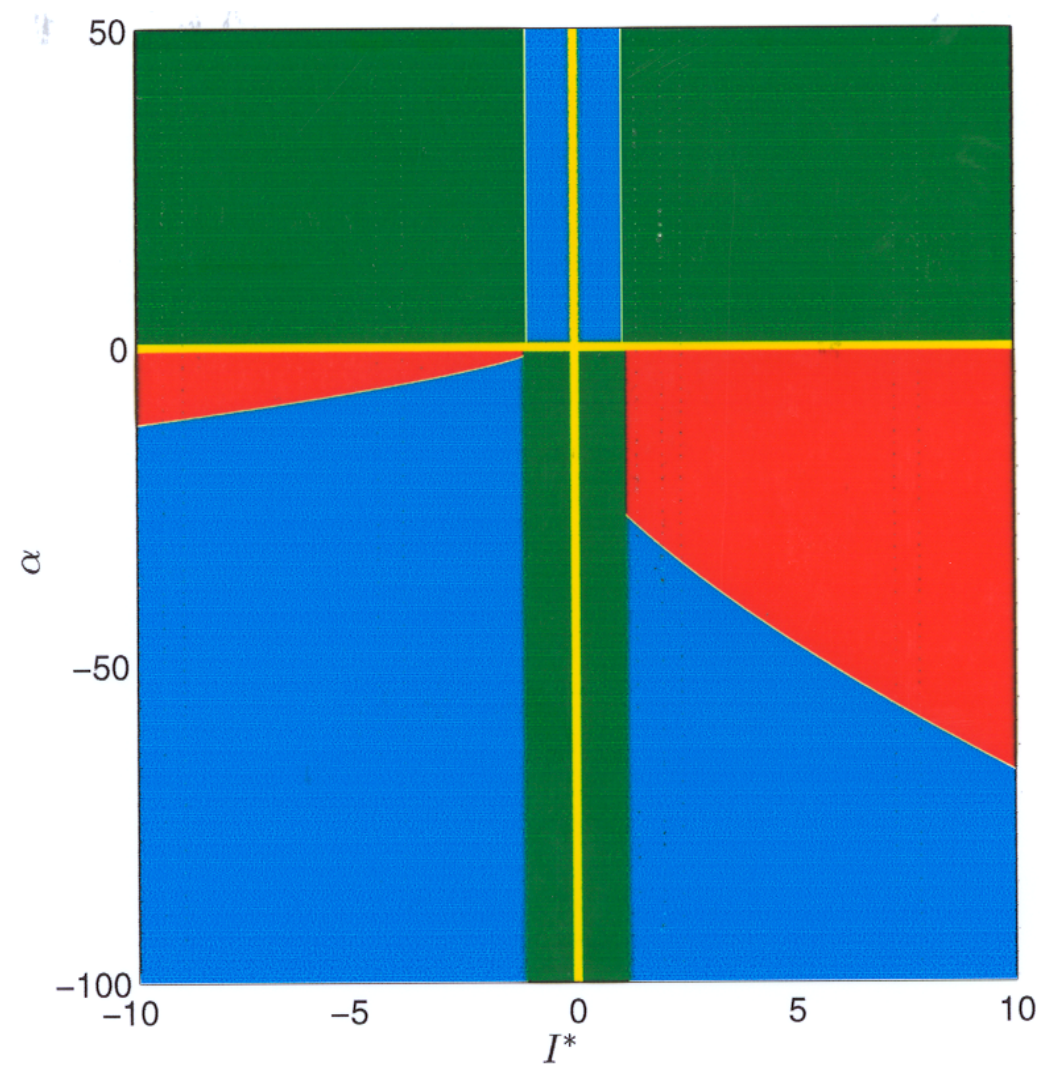

Fig. 19. Parameter space in the $I^{*}-\alpha$ plane. The fixed parameters are $\gamma=0, a=-1$ and $c=2$. The local active regions and the restricted local passive regions are colored in green and cyan, respectively. The edge-of-chaos regions are shown in red. 


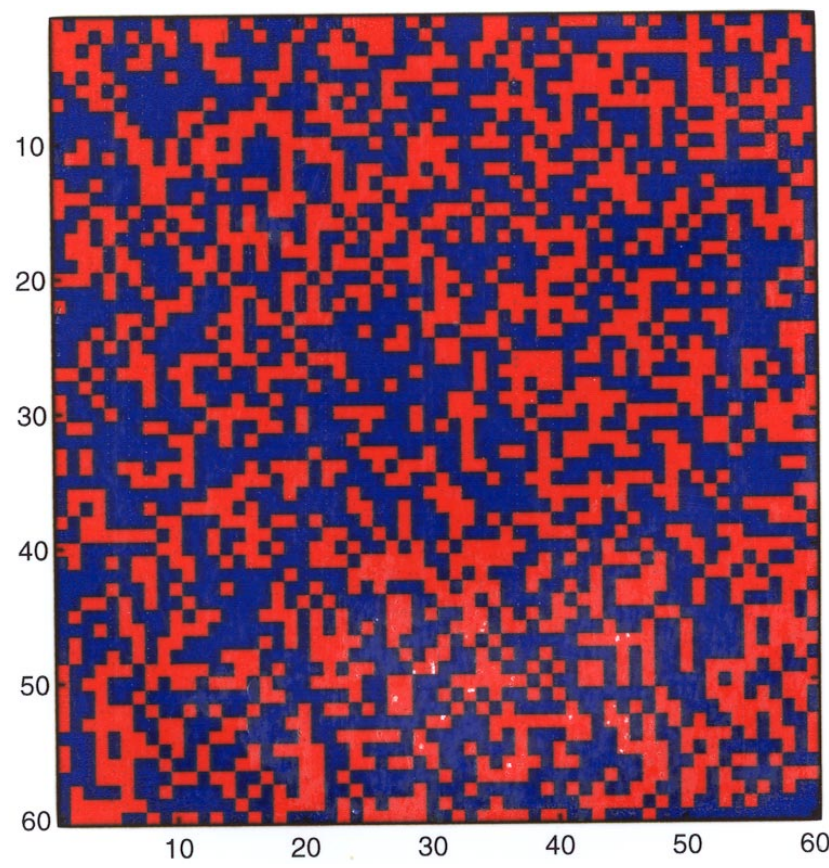

(a)

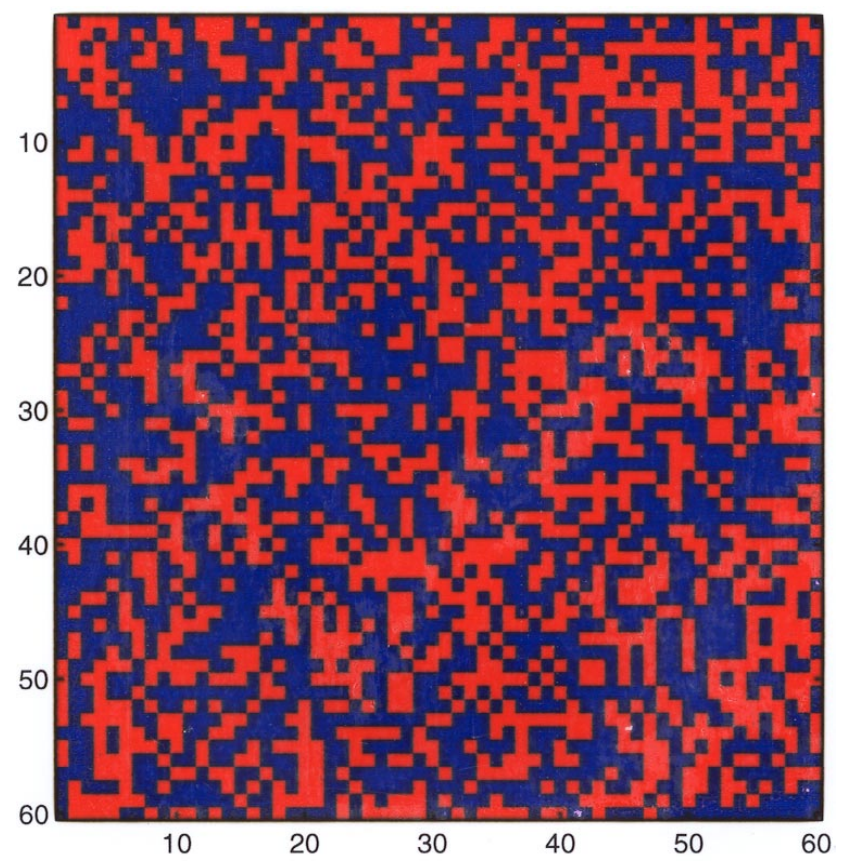

(c)

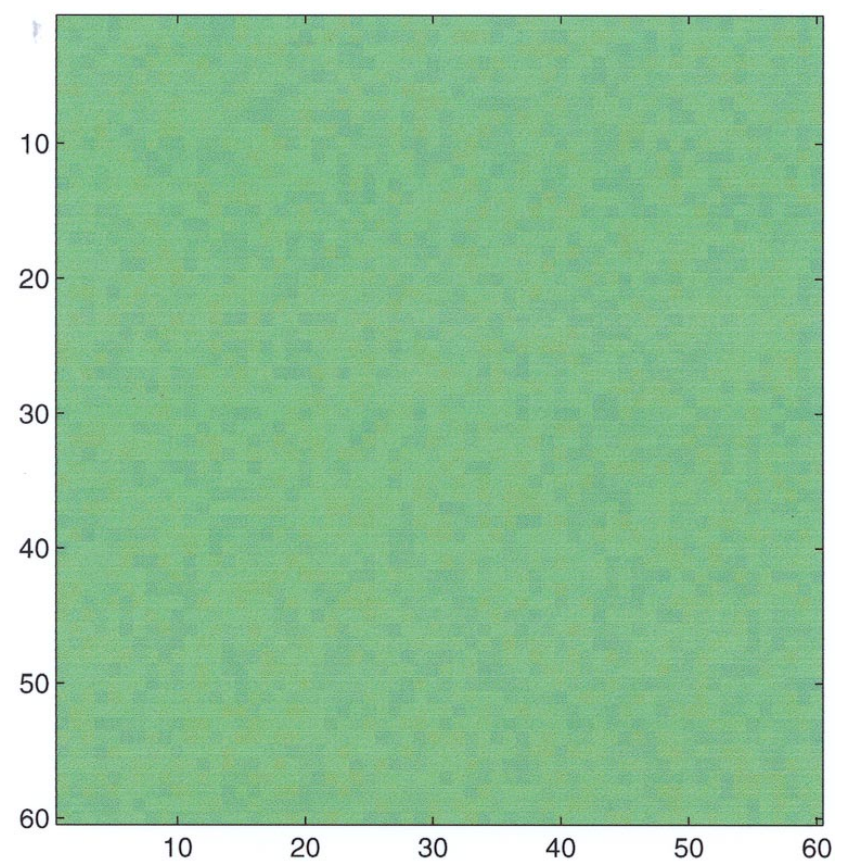

(b)

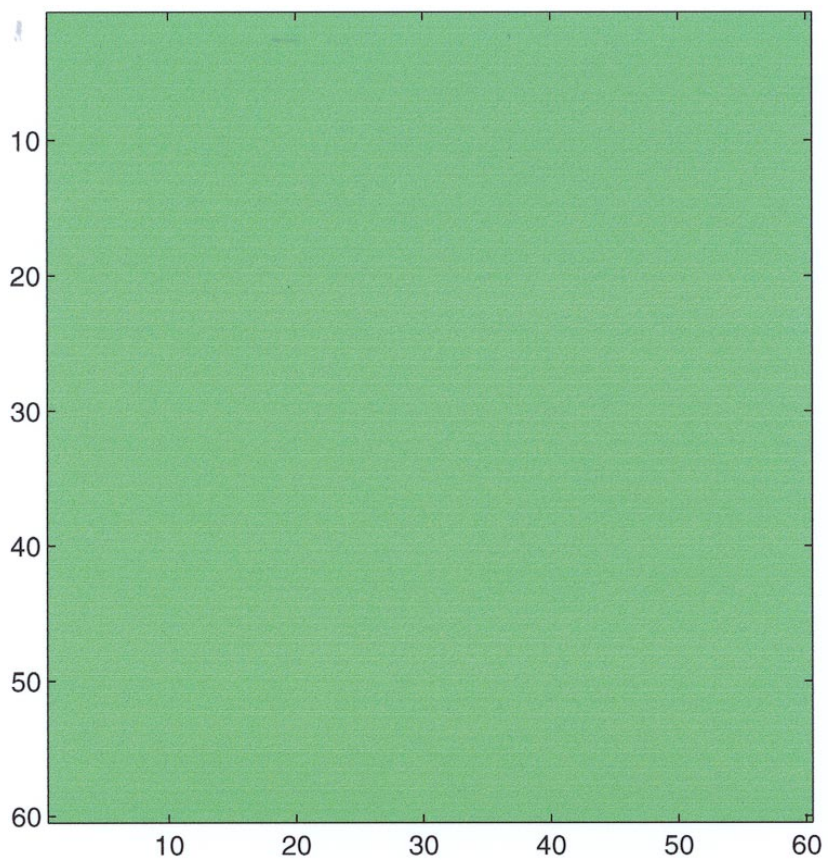

(d)

Fig. 20. Simulation results of Chua's circuit CNN with parameter $\alpha=-50, \beta=14, \gamma=0, a=-1, c=2, D_{1}=0.5$ and $D_{2}=0.5$. (a) $V_{1}(5)$. (b) $V_{2}(5)$. (c) $V_{1}(10)$. (d) $V_{2}(10)$. 
(b) Fix $D_{1}$ and $D_{2}$ and initial condition, and with small values of $\alpha$, we observe the CNN settles down to a trivial homogeneous pattern. However, upon increasing $\alpha$ beyond some critical value, we find the CNN becomes unstable.

The edge-of-chaos region (shown in red) in Fig. 19 implies some possibilities of pattern formation by this CNN.

10.2.1. $\alpha=-50, \beta=14, \gamma=0$, $a=-1, c=2$

$D_{1}=0.5$ and $D_{2}=0.5$

Figure 20 shows our simulation results with parameter $\alpha=-50, \beta=14, \gamma=0, a=-1, c=2$, $D_{1}=0.5$ and $D_{2}=0.5$. The array size of this $\mathrm{CNN}$ is $60 \times 60$. Observe that $V_{1}(t)$ converges to a static binary pattern while $V_{2}(t)$ converges to a homogeneous pattern.

$$
\text { 10.2.2. } \quad \begin{aligned}
& \alpha=-0.1, \beta=14, \gamma=0, \\
& \\
& a=-1, c=2
\end{aligned}
$$

$D_{1}=0.5$ and $D_{2}=0.5$

Figure 21 shows our simulation results with param-

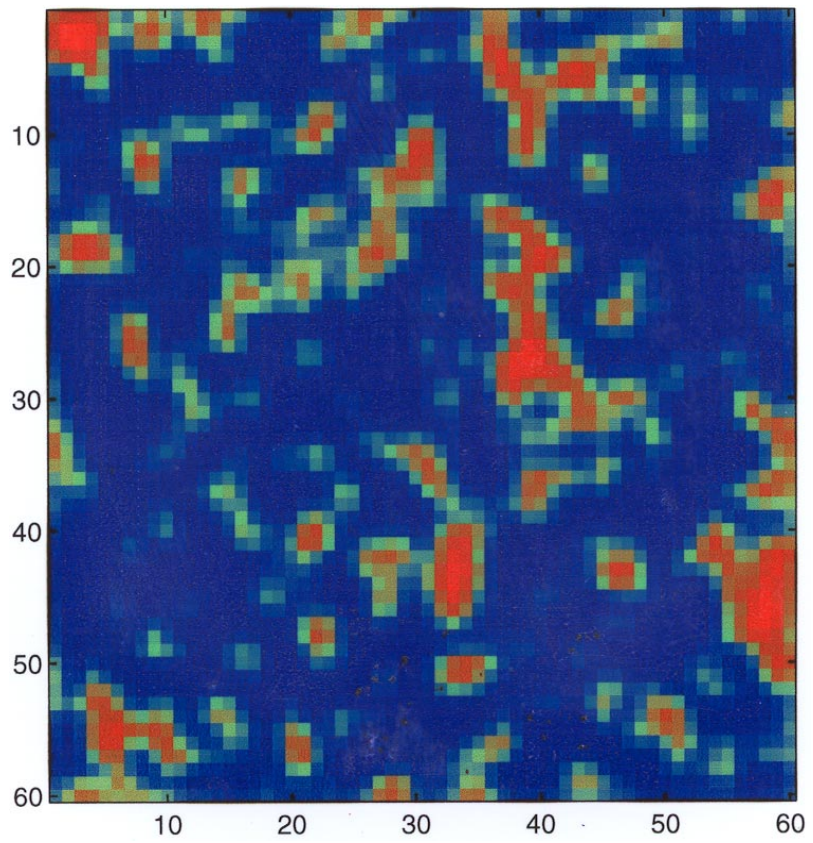

(a) eter $\alpha=-0.1, \beta=14, \gamma=0, a=-1, c=2$, $D_{1}=0.5$ and $D_{2}=0.5$. The array size of this CNN is $60 \times 60$. Observe that $V_{1}(t)$ generates a long series of transient patch patterns but it eventually converges to a homogeneous pattern, while $V_{2}(t)$ converges rapidly to a homogeneous pattern almost from the beginning.

$D_{1}=5$ and $D_{2}=5$

When the coupling becomes stronger we find that many cells converge to homogeneous patterns except the boundary cells. The simulation results are shown in Fig. 22.

$D_{1}=0.1$ and $D_{2}=0.1$

The simulation results corresponding to weak couplings are shown in Fig. 23. Observe that although the CNN settled down to a binary pattern rapidly, the pattern is "random-like" because the couplings between cells are too small to achieve any local synchronization. Hence, big patches cannot last for a relatively long period of time.

$D_{1}=100$ and $D_{2}=100$

The simulation in Fig. 24 shows the effect of strong couplings. Observe that $V_{1}(t)$ converges to a static pattern consisting of a blue region and a red region

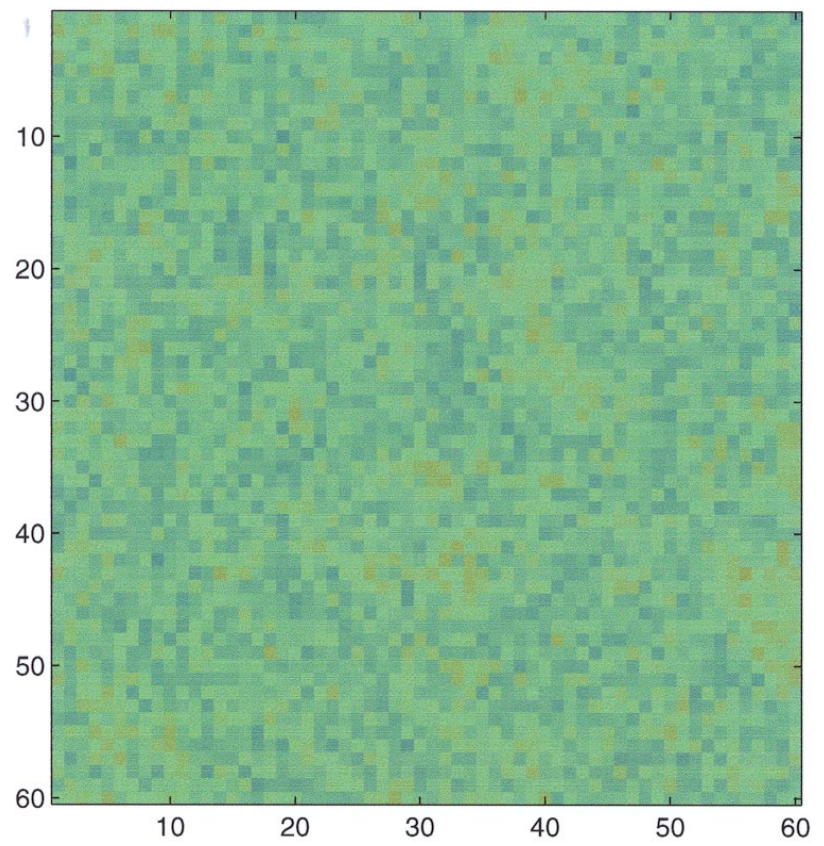

(b)

Fig. 21. Simulation results of Chua's circuit CNN with parameter $\alpha=-0.1, \beta=14, \gamma=0, a=-1, c=2, D_{1}=0.5$ and $D_{2}=0.5$. (a) $V_{1}(2)$. (b) $V_{2}(2)$. (c) $V_{1}(4)$. (d) $V_{2}(4)$. (e) $V_{1}(5)$. (f) $V_{2}(5)$. (g) $V_{1}(10)$. (h) $V_{2}(10)$. (i) $V_{1}(20) . \quad\left(\right.$ j) $V_{2}(20)$. (k) $V_{1}(30)$. (l) $V_{2}(30)$. (m) $V_{1}(40)$. (n) $V_{2}(40)$. (o) $V_{1}(60)$. (p) $V_{2}(60)$. 


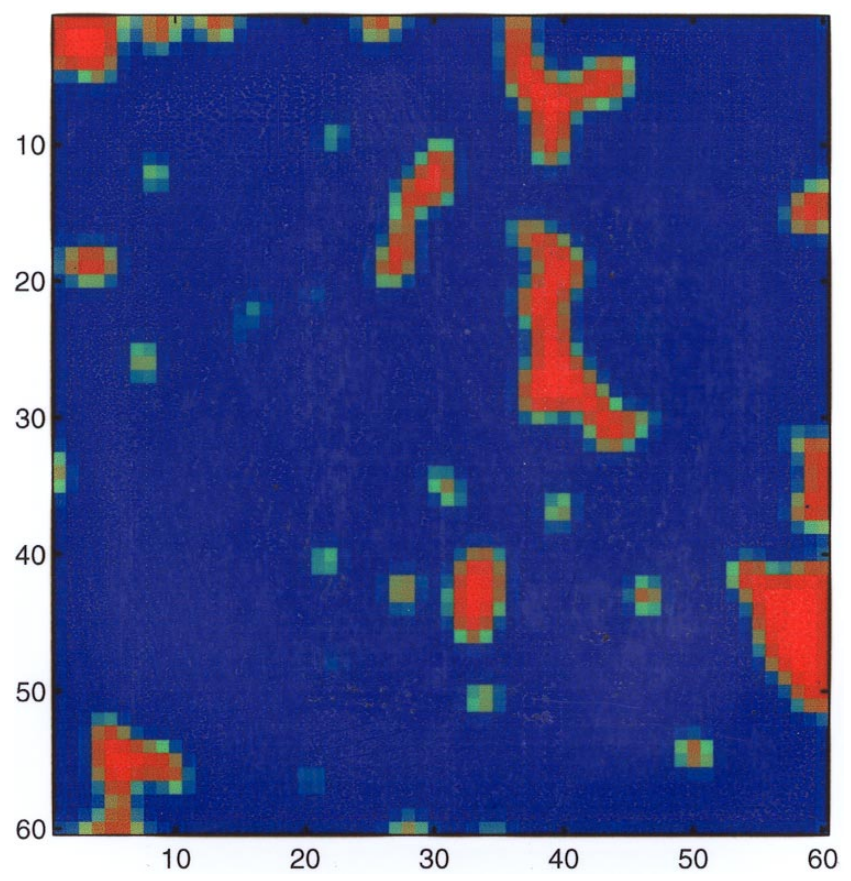

(c)

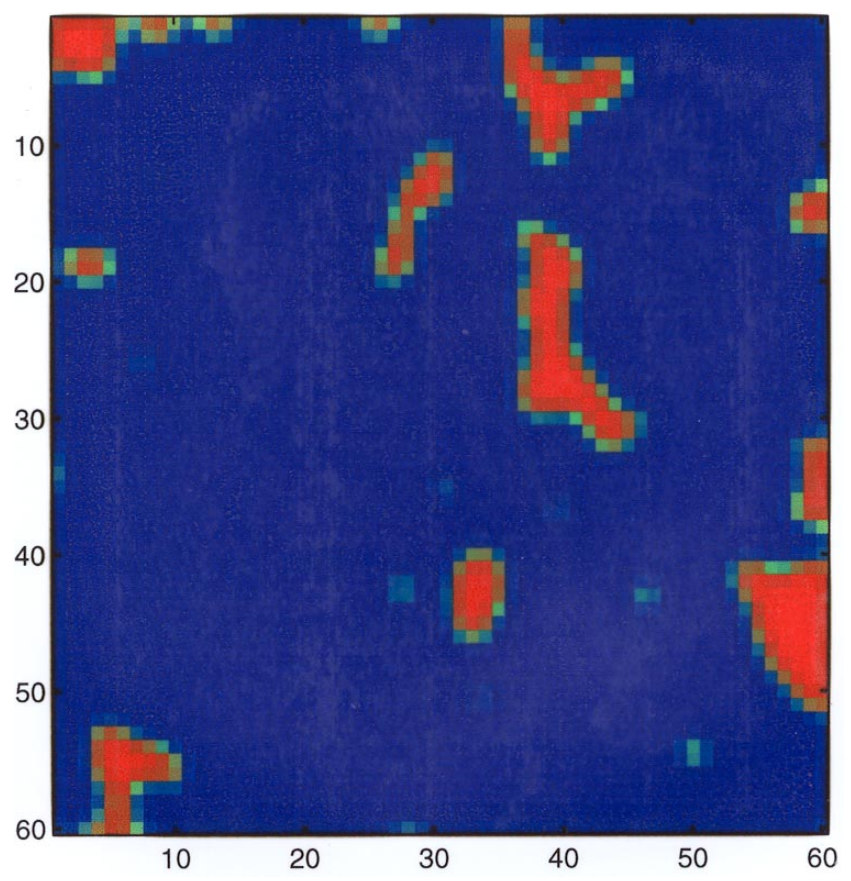

(e)

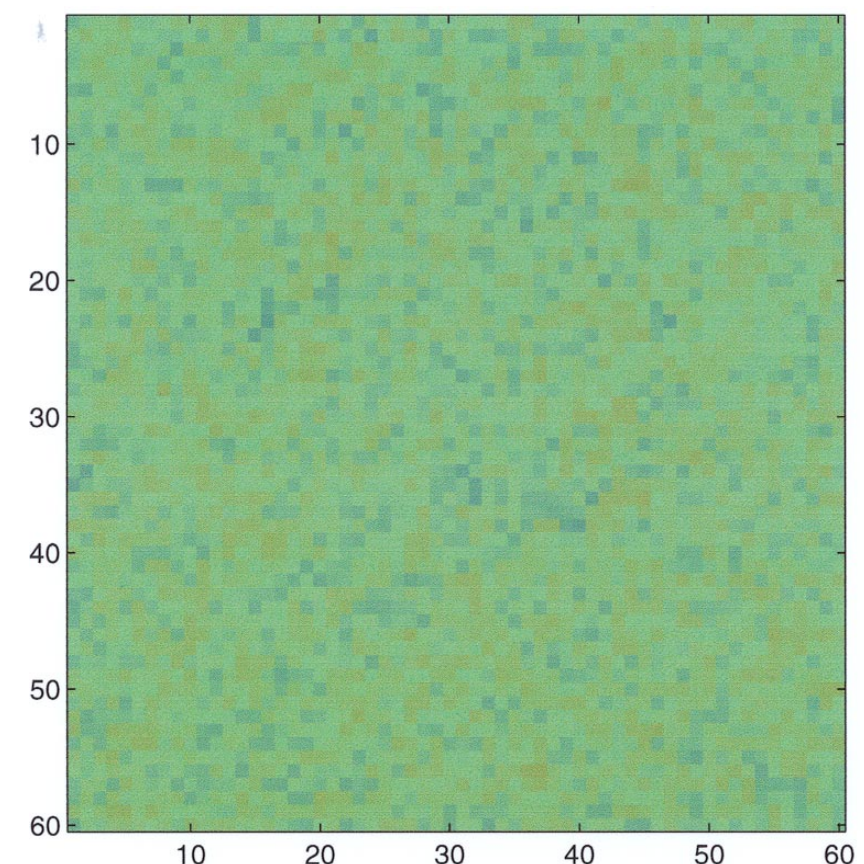

(d)

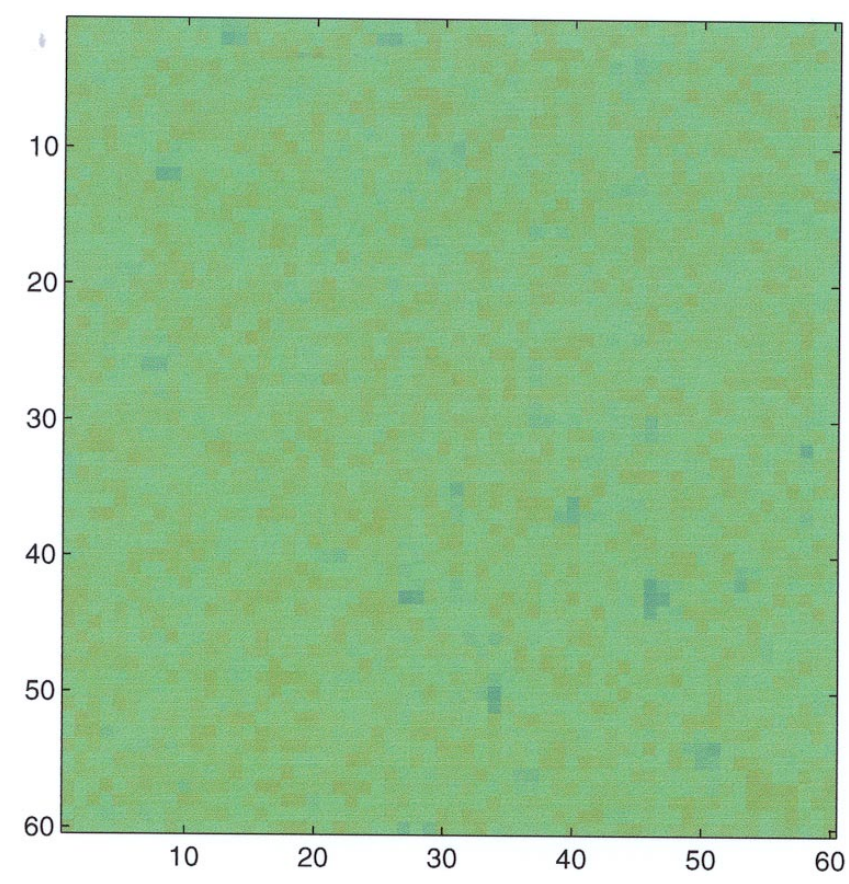

(f)

Fig. 21. (Continued) 


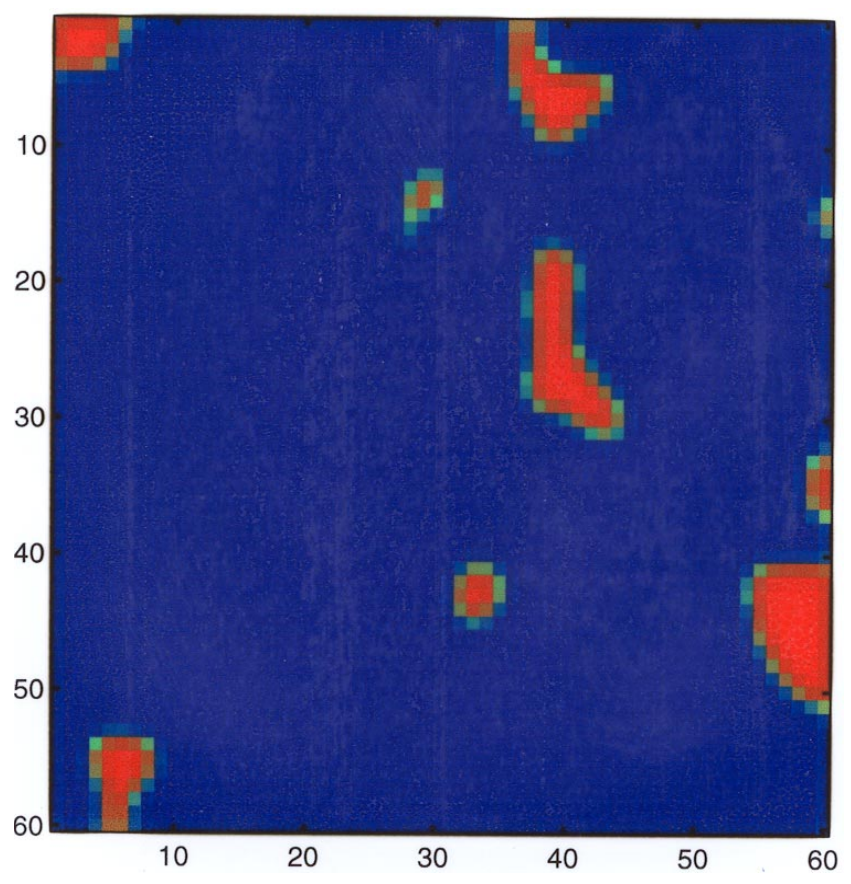

(g)

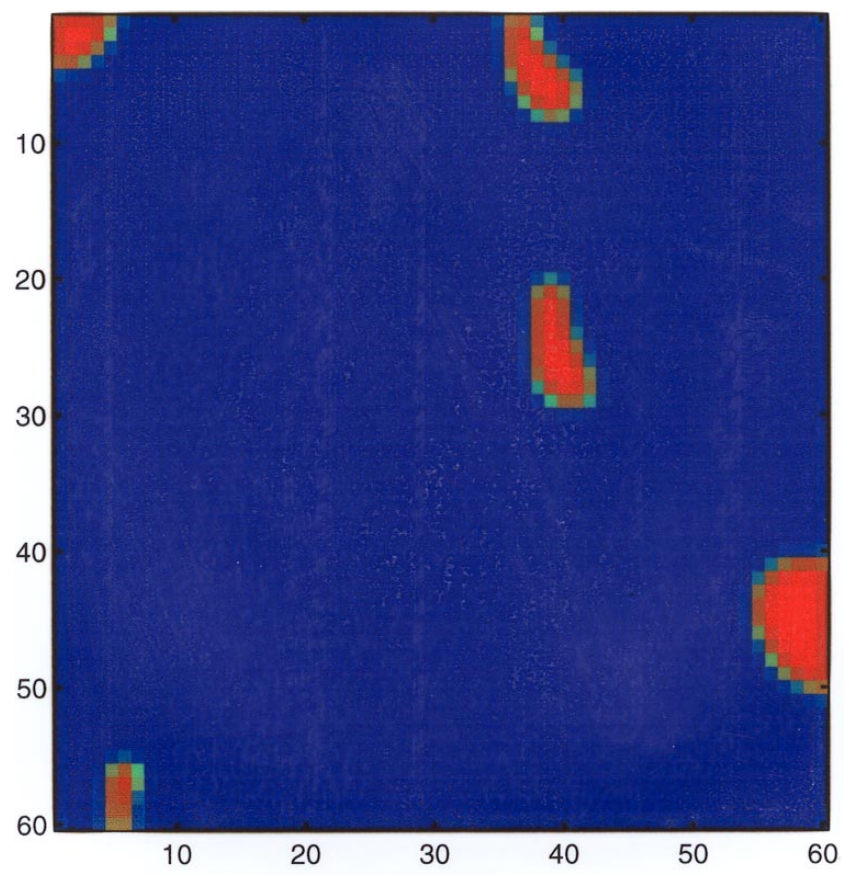

(i)

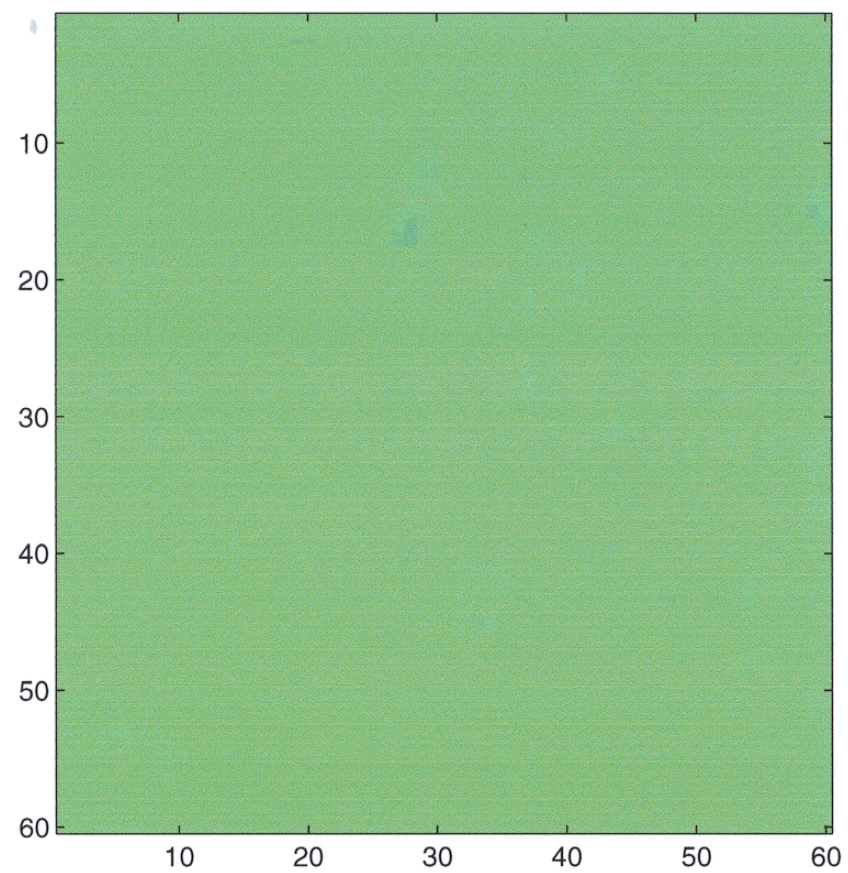

(h)

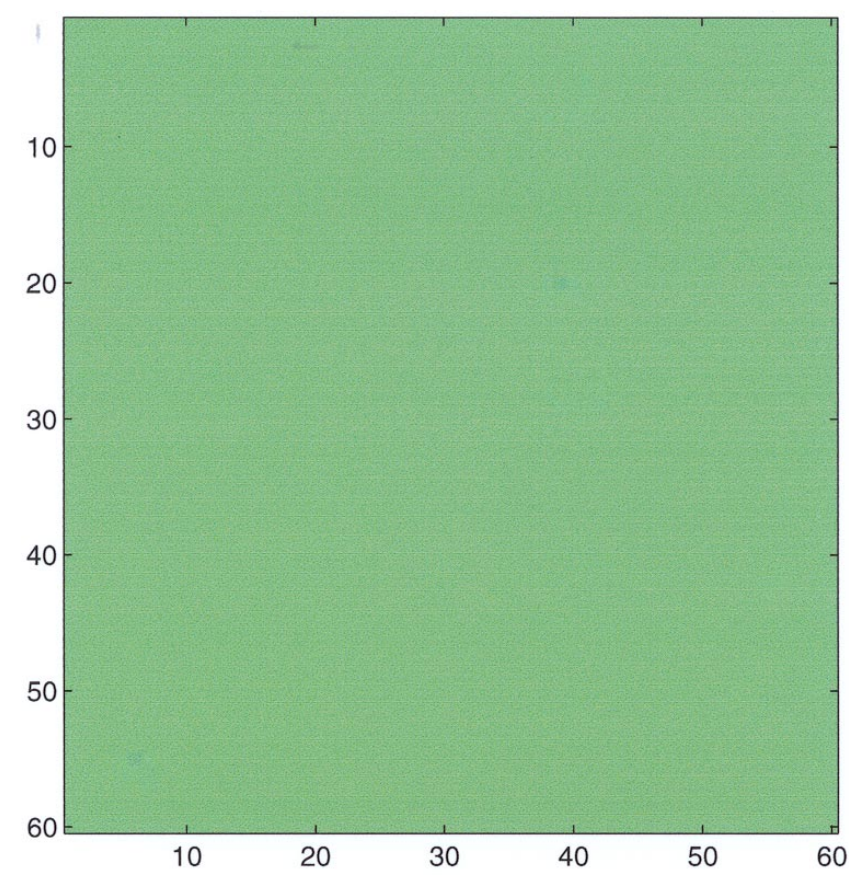

(j)

Fig. 21. (Continued) 


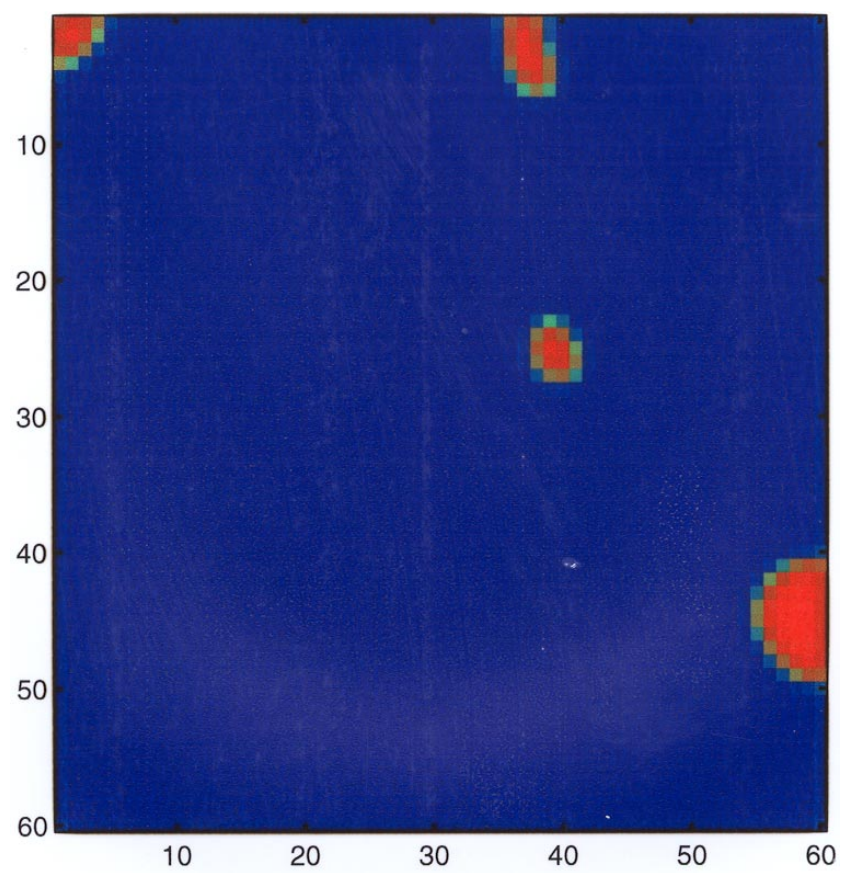

(k)

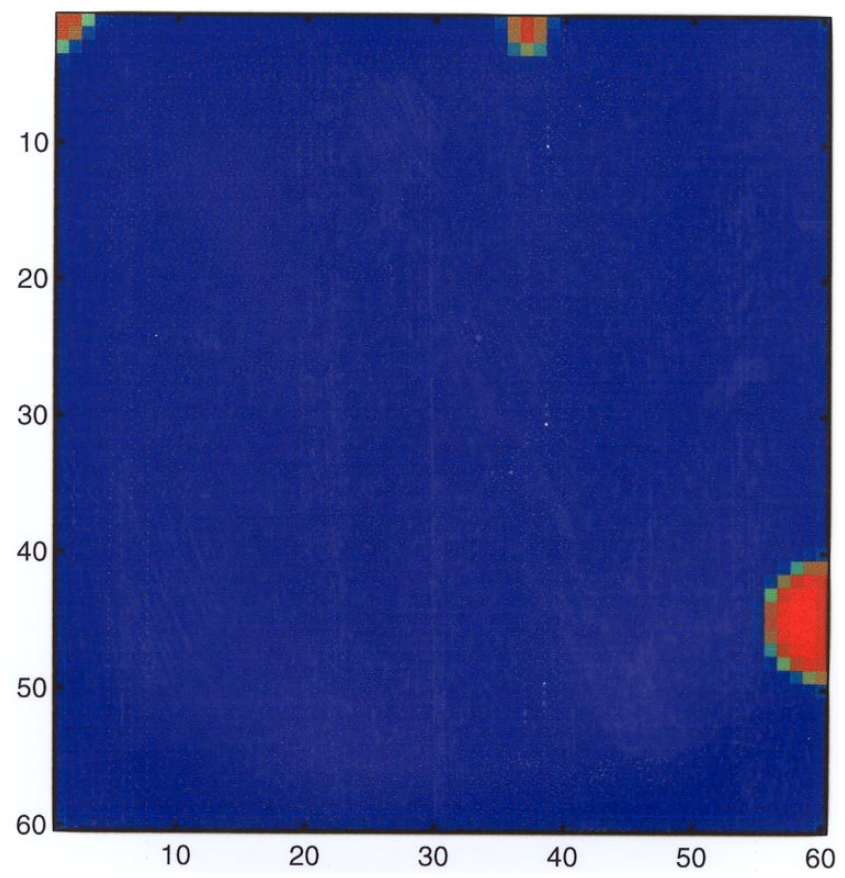

$(\mathrm{m})$

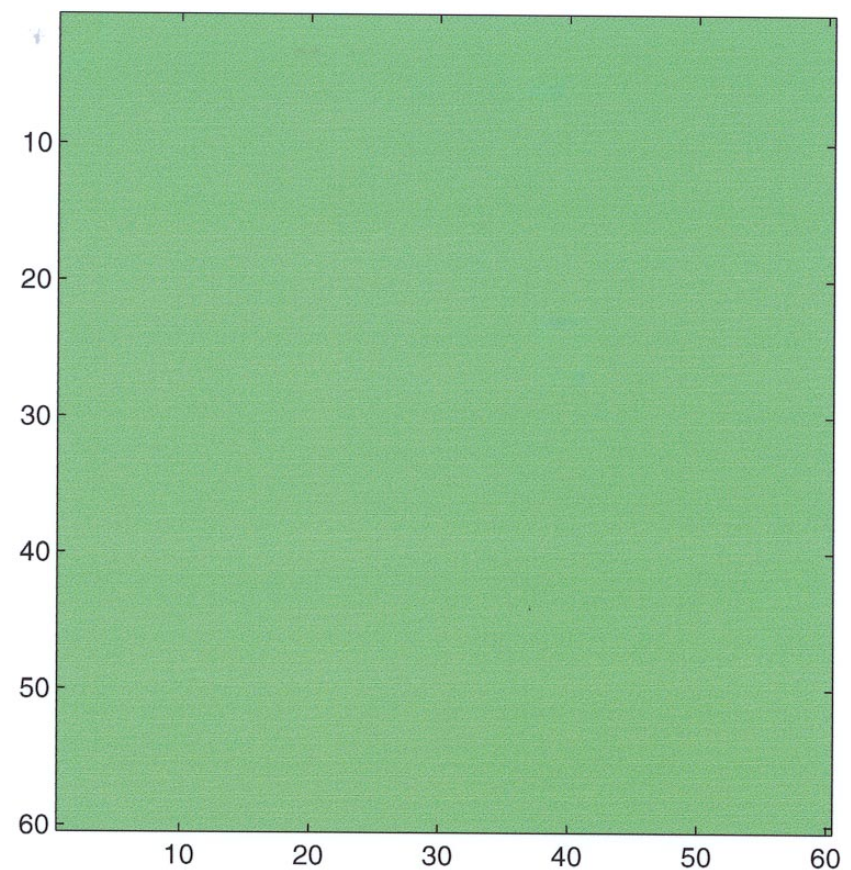

(1)

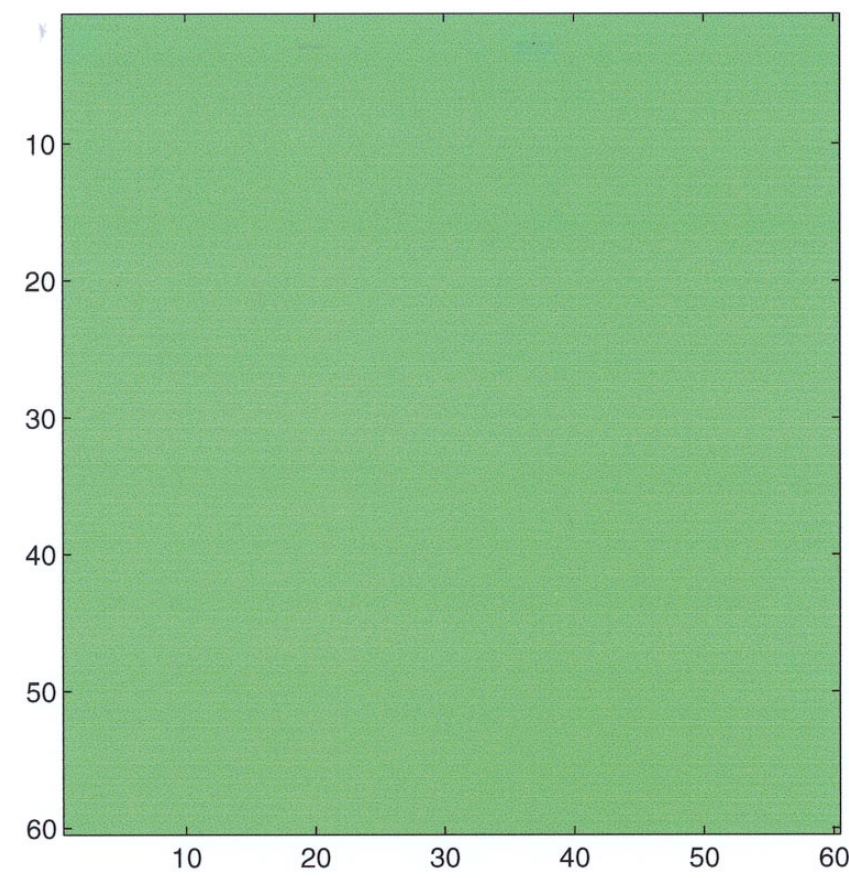

(n)

Fig. 21. (Continued) 


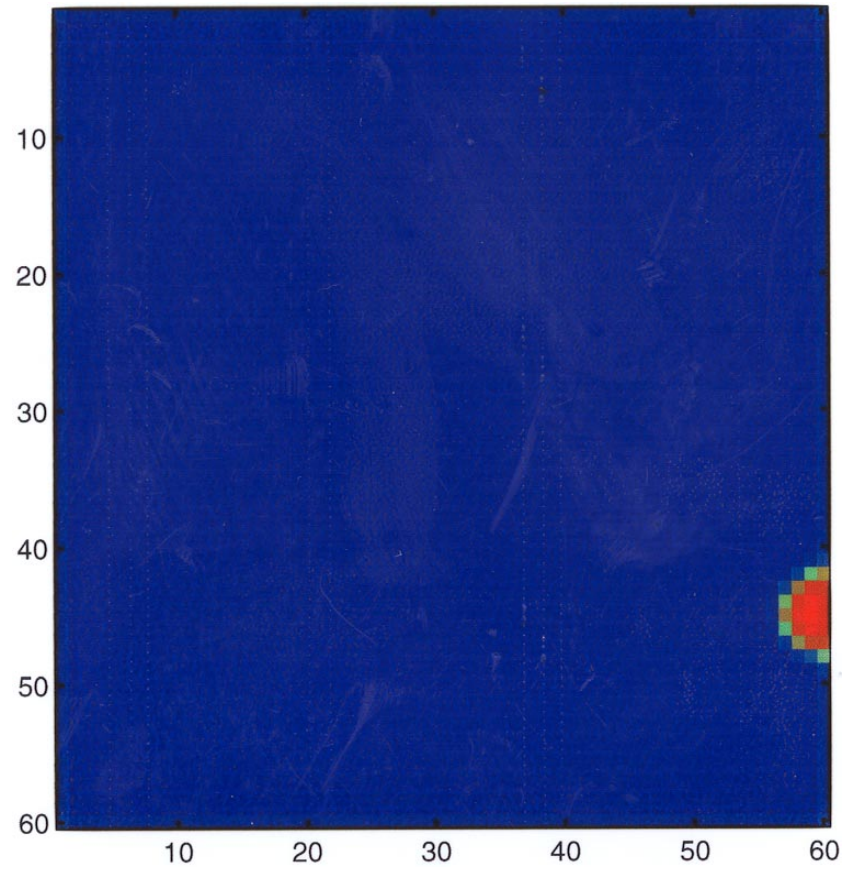

(o)

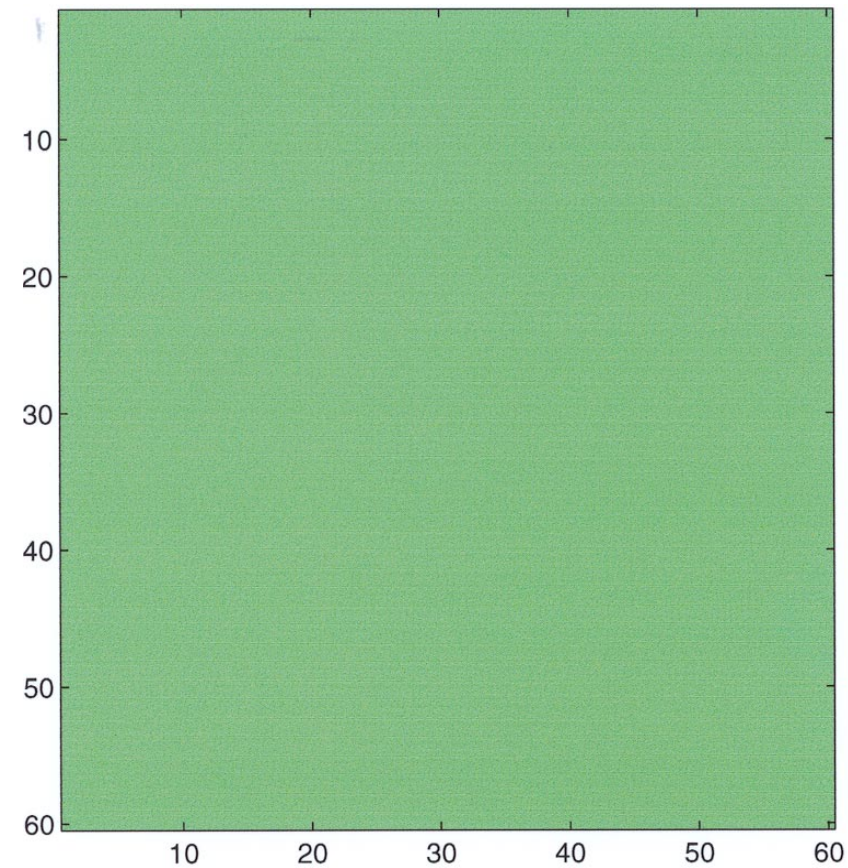

(p)

Fig. 21. (Continued)

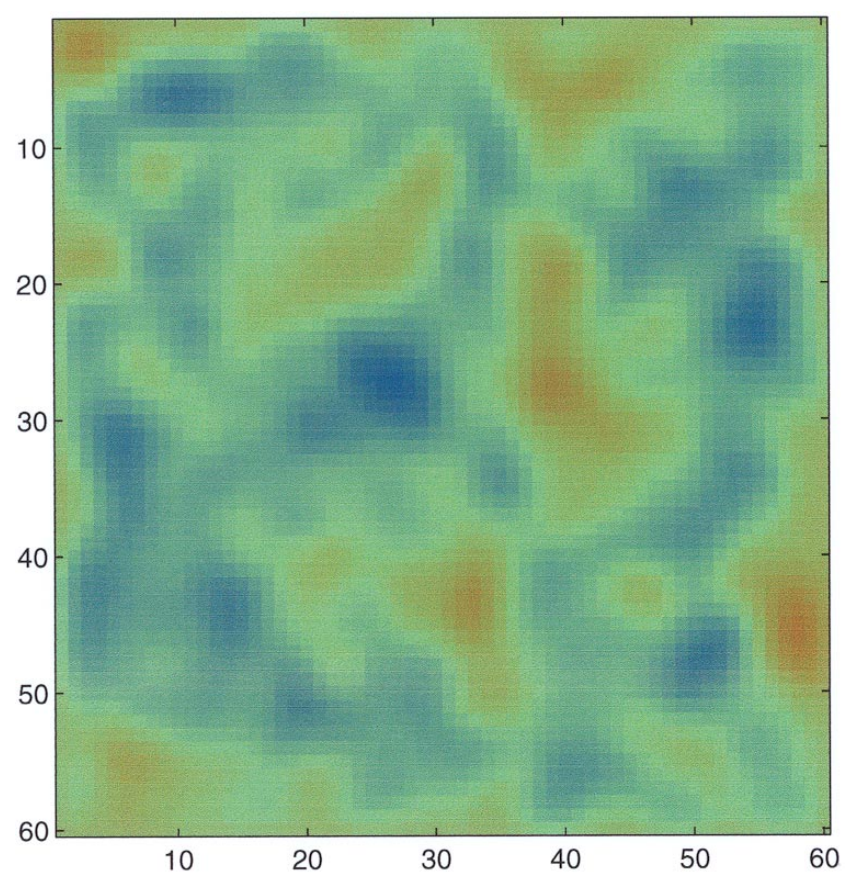

(a)

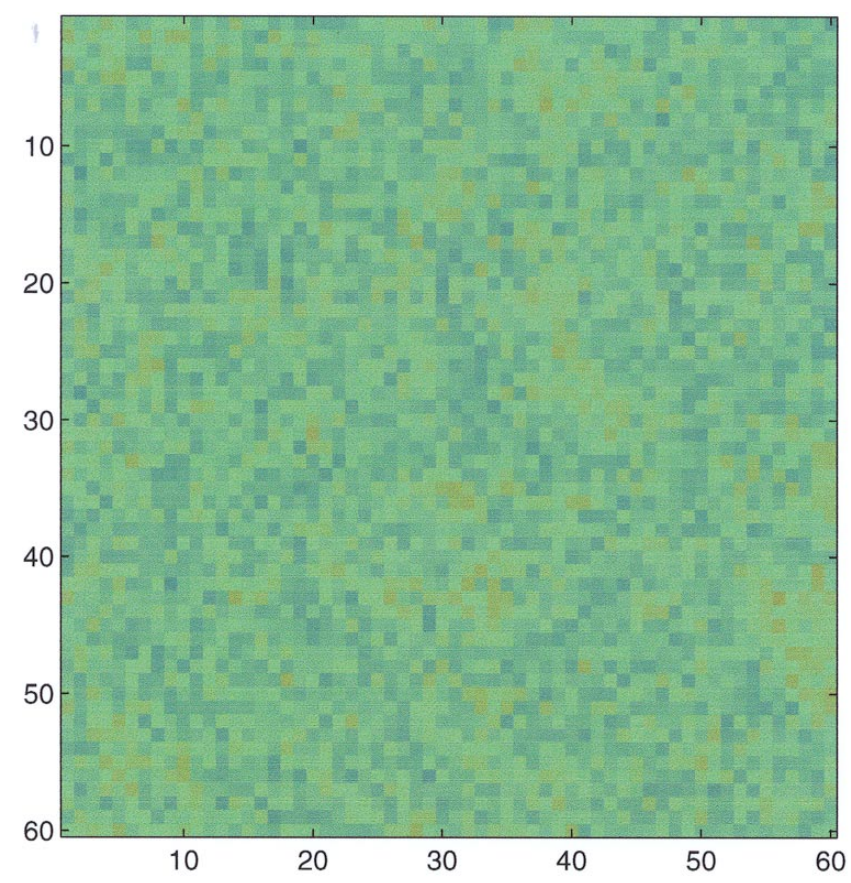

(b)

Fig. 22. Simulation results of Chua's circuit CNN with parameter $\alpha=-0.1, \beta=14, \gamma=0, a=-1, c=2, D_{1}=5$ and $D_{2}=5$. (a) $V_{1}(0.5)$. (b) $V_{2}(0.5)$. (c) $V_{1}(1)$. (d) $V_{2}(1)$. (e) $V_{1}(2)$. (f) $V_{2}(2)$. (g) $V_{1}(4)$. (h) $V_{2}(4) . \quad$ (i) $V_{1}(5) . \quad$ (j) $V_{2}(5)$. (k) $V_{1}(10)$. (l) $V_{2}(10)$. 


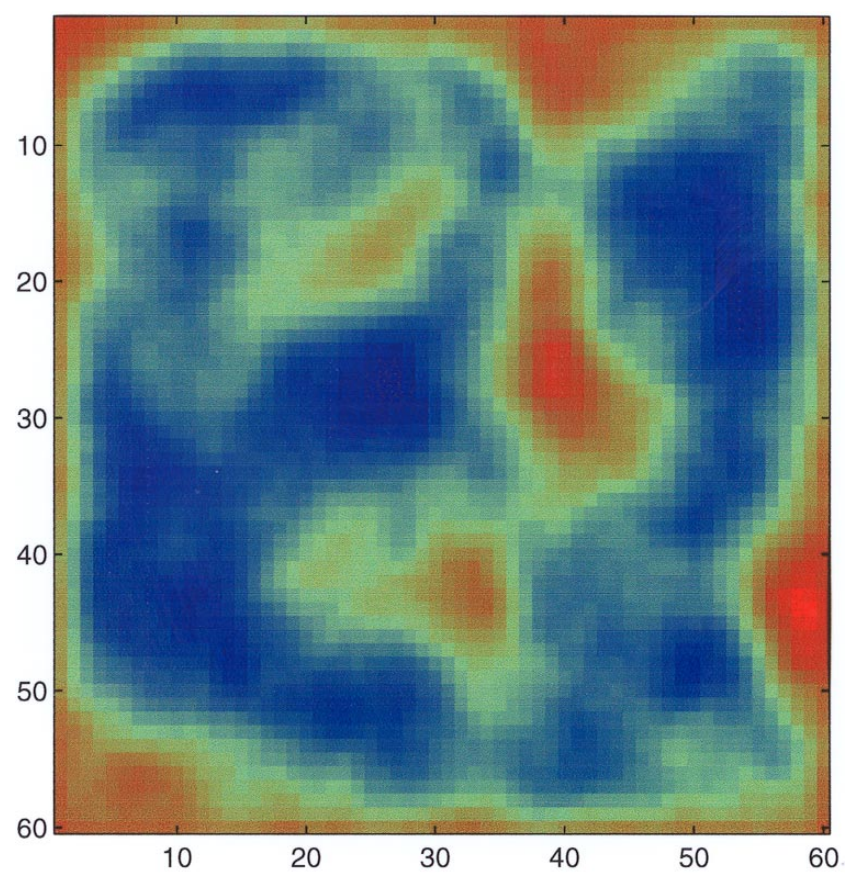

(c)

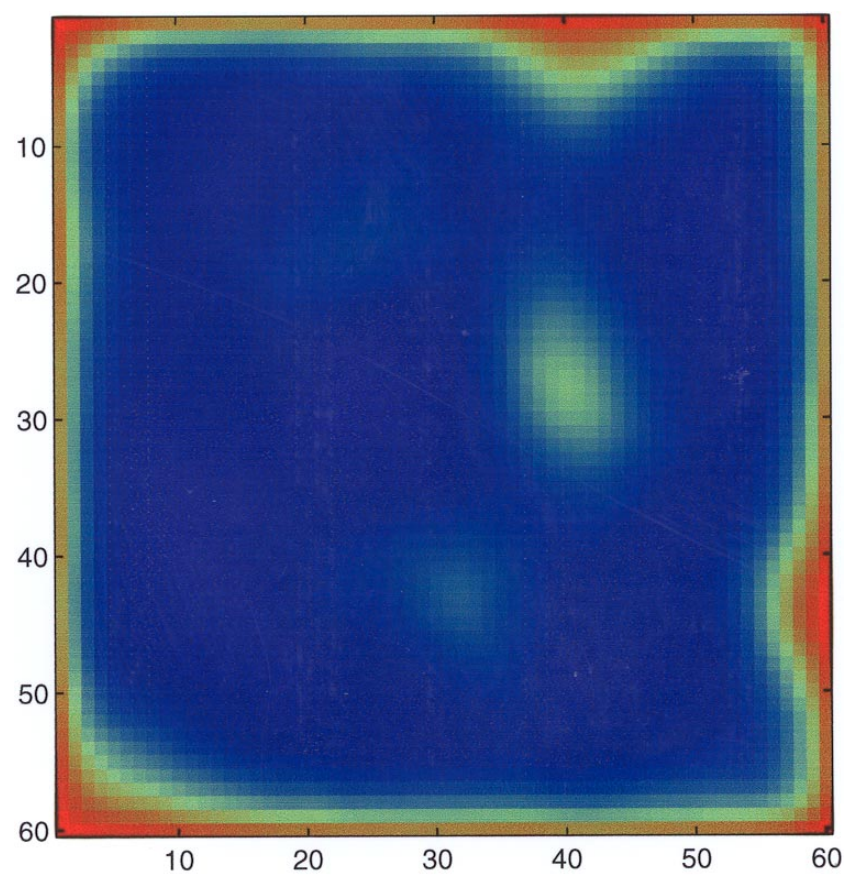

(e)

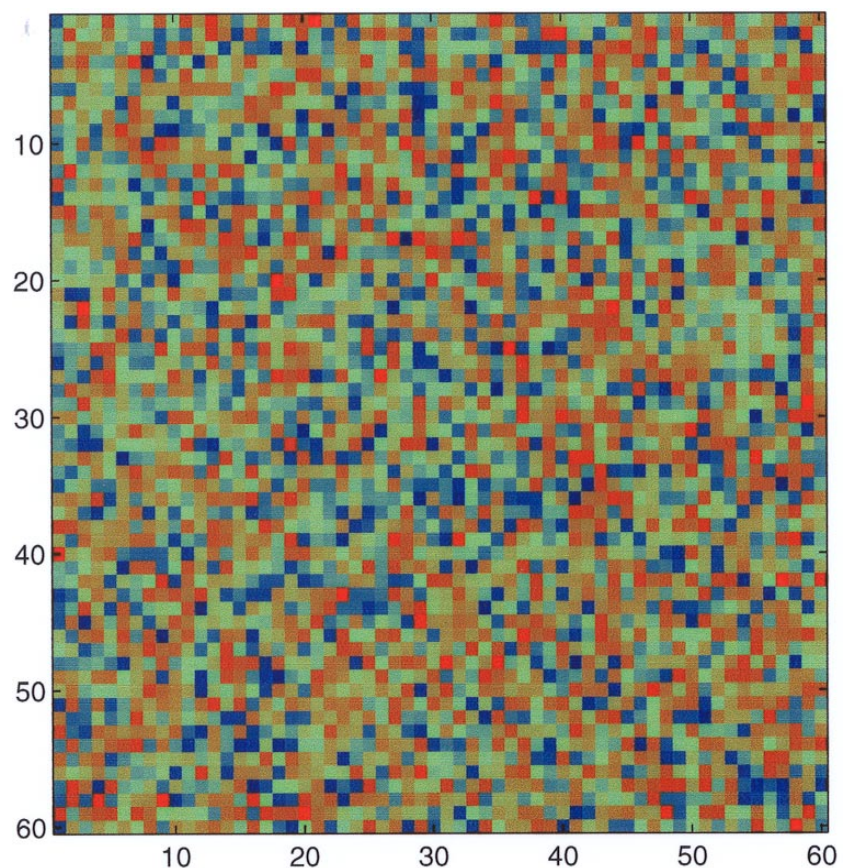

(d)

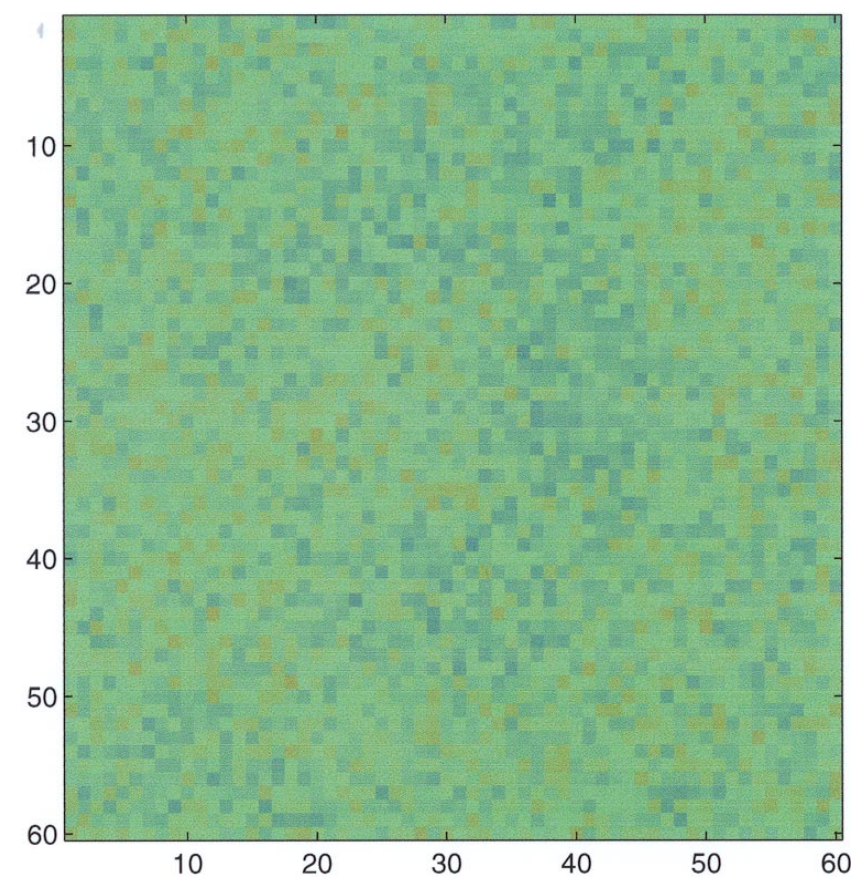

(f)

Fig. 22. (Continued) 


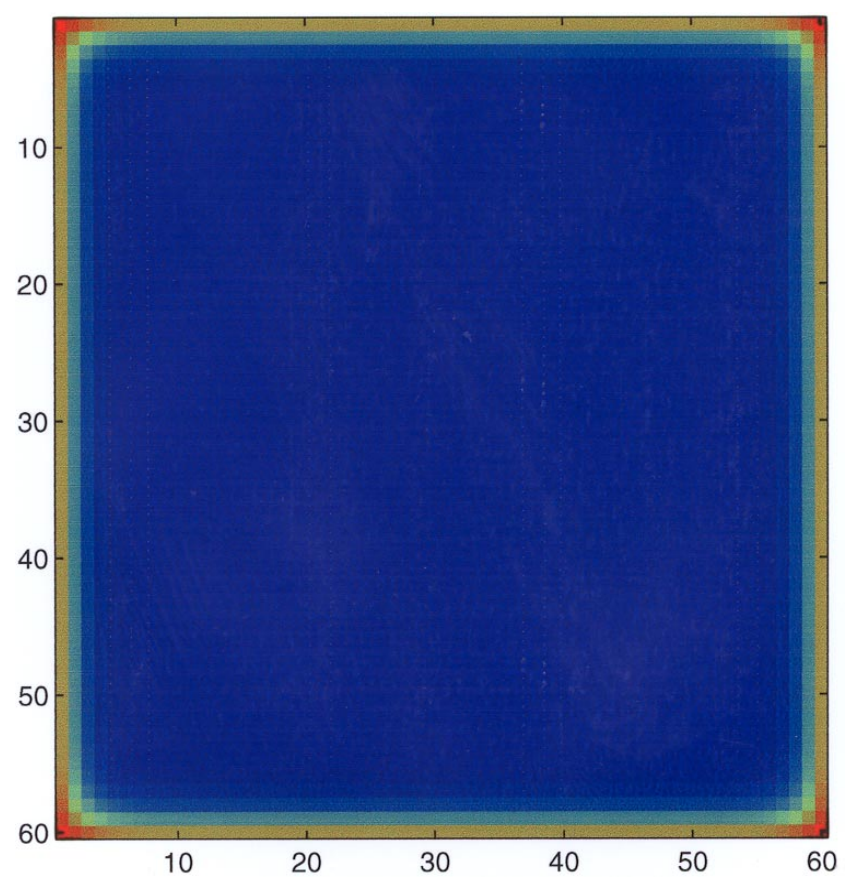

(g)

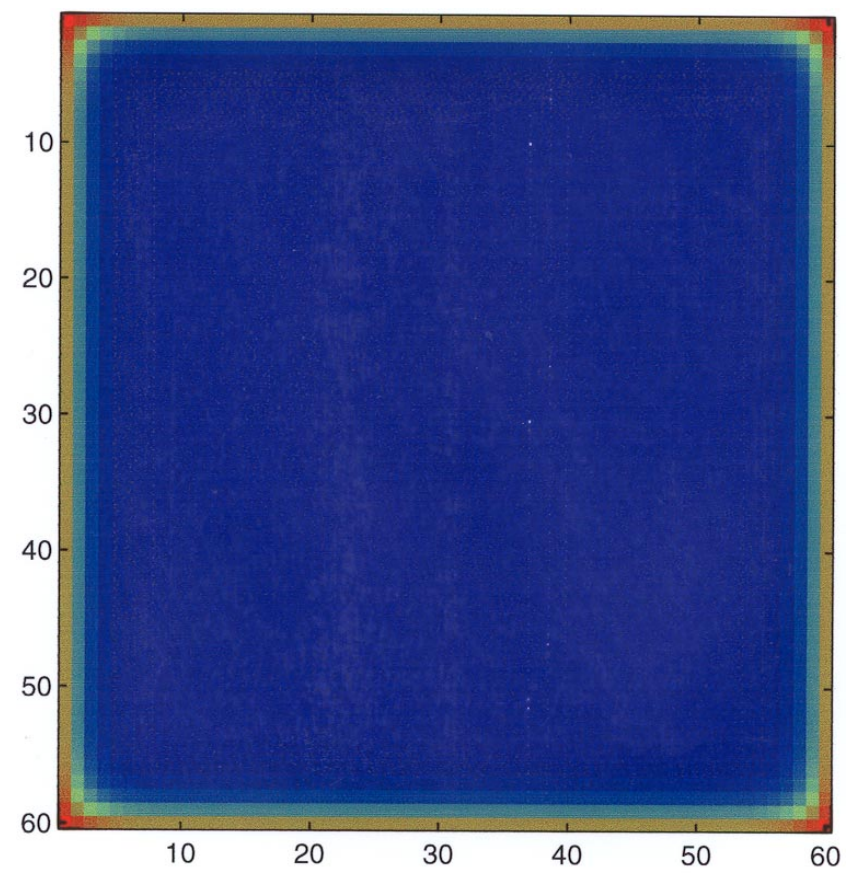

(i)

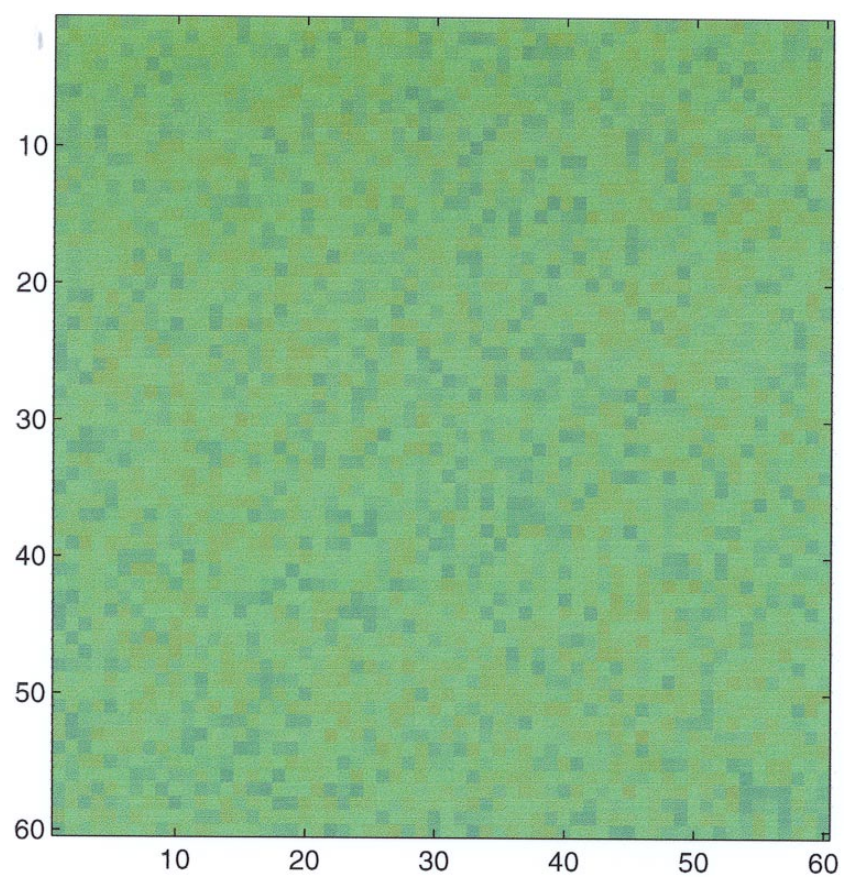

(h)

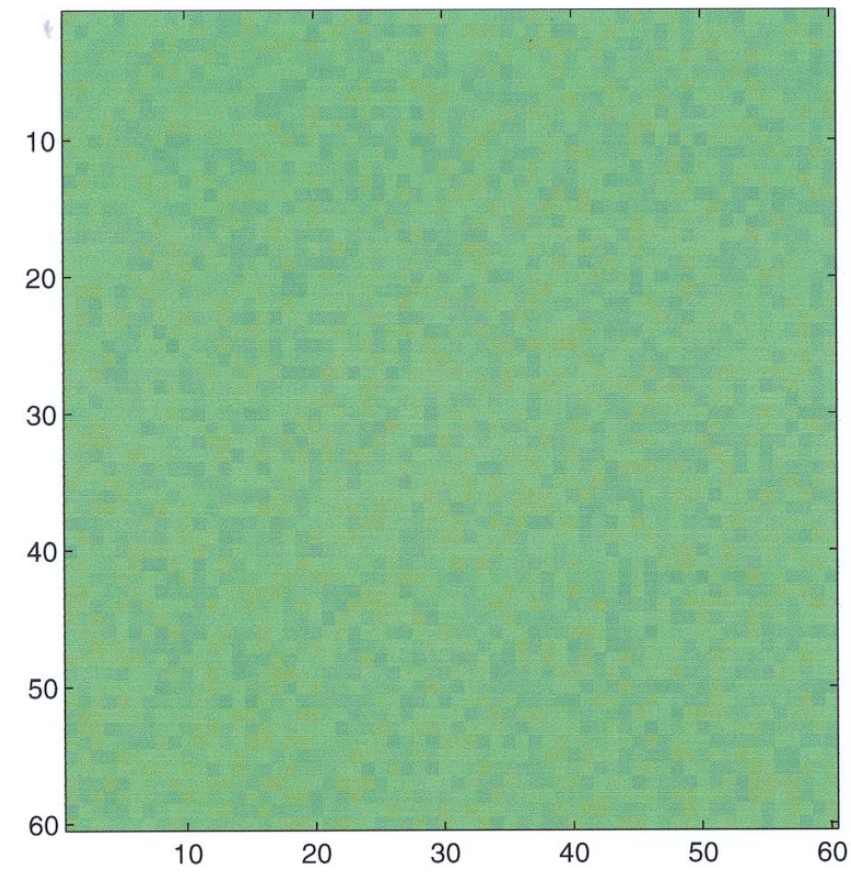

(j)

Fig. 22. (Continued) 


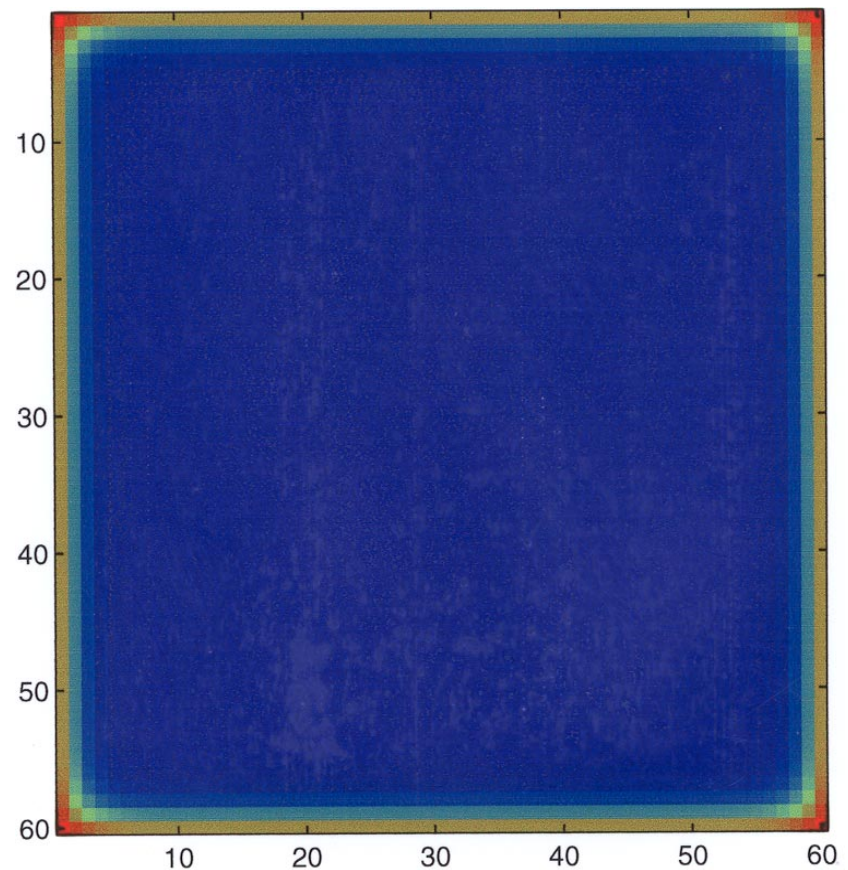

$(\mathrm{k})$

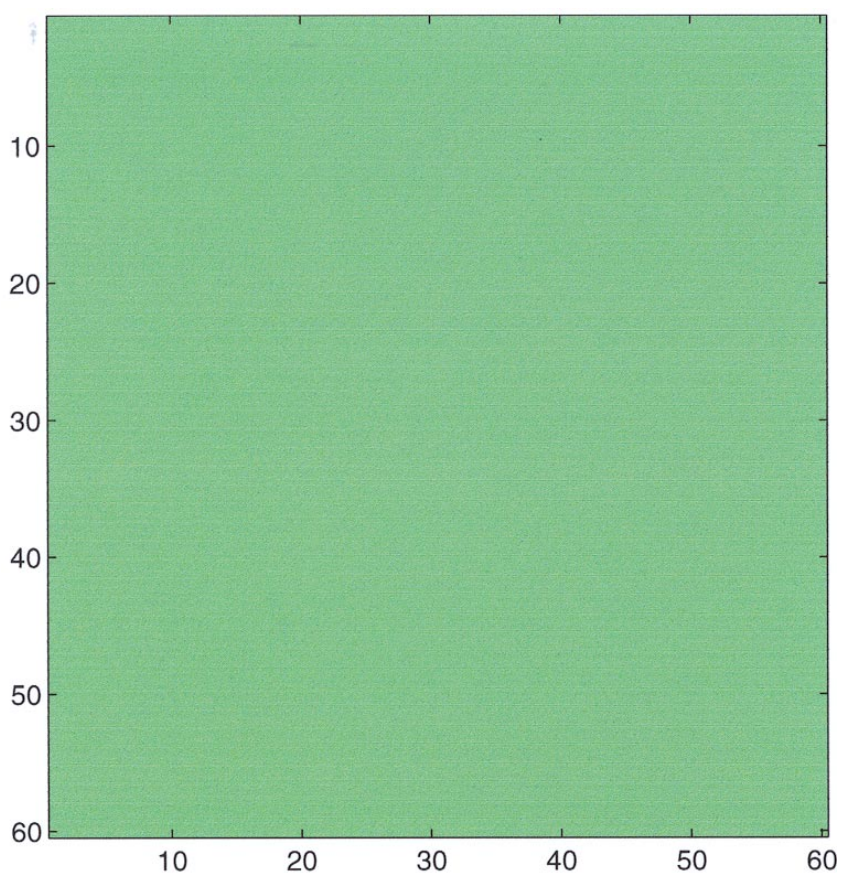

(1)

Fig. 22. (Continued)

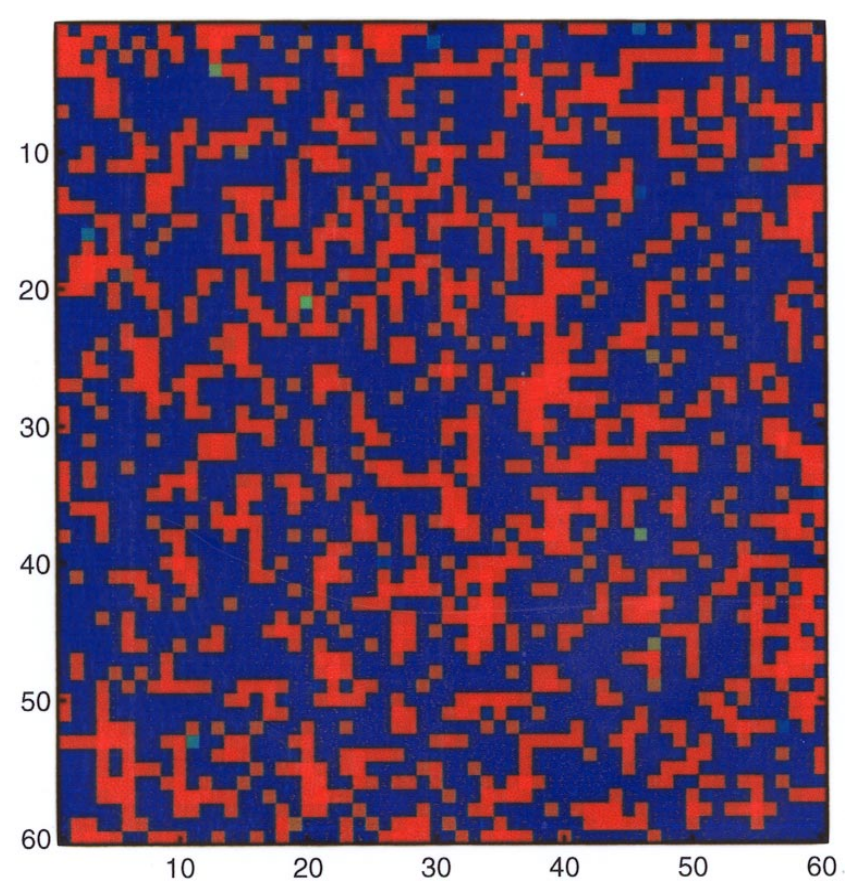

(a)

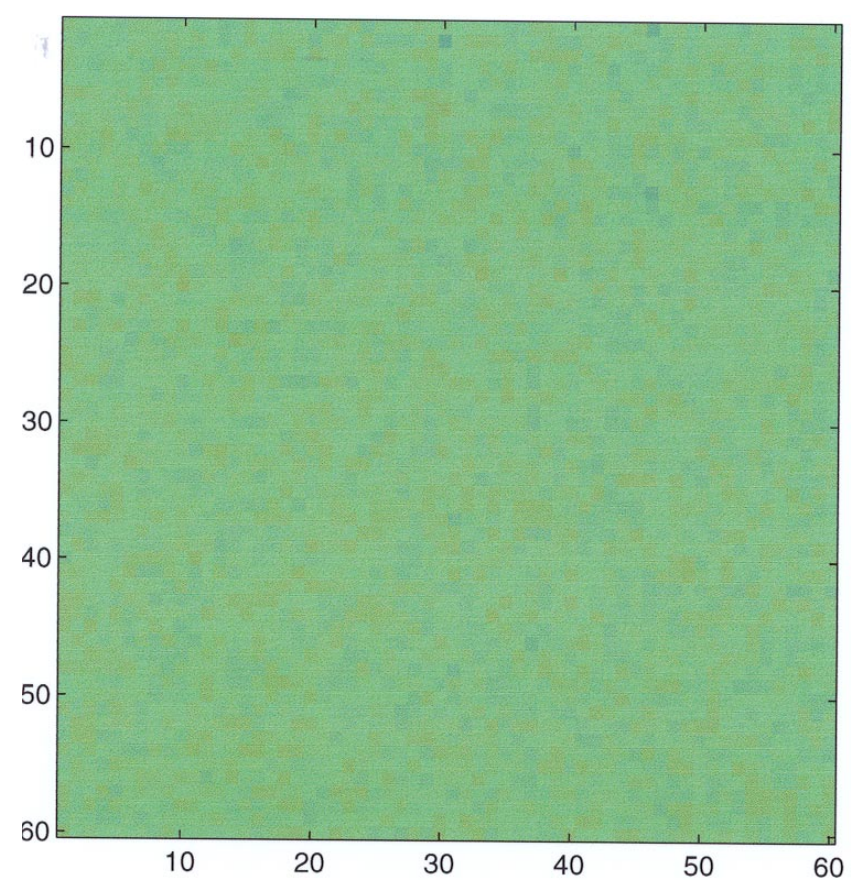

(b)

Fig. 23. Simulation results of Chua's circuit CNN with parameter $\alpha=-0.1, \beta=14, \gamma=0, a=-1, c=2, D_{1}=0.1$ and $D_{2}=0.1$. (a) $V_{1}(5)$. (b) $V_{2}(5)$. (c) $V_{1}(10)$. (d) $V_{2}(10)$. (e) $V_{1}(20)$. (f) $V_{2}(20)$. (g) $V_{1}(40)$. (h) $V_{2}(40)$. 


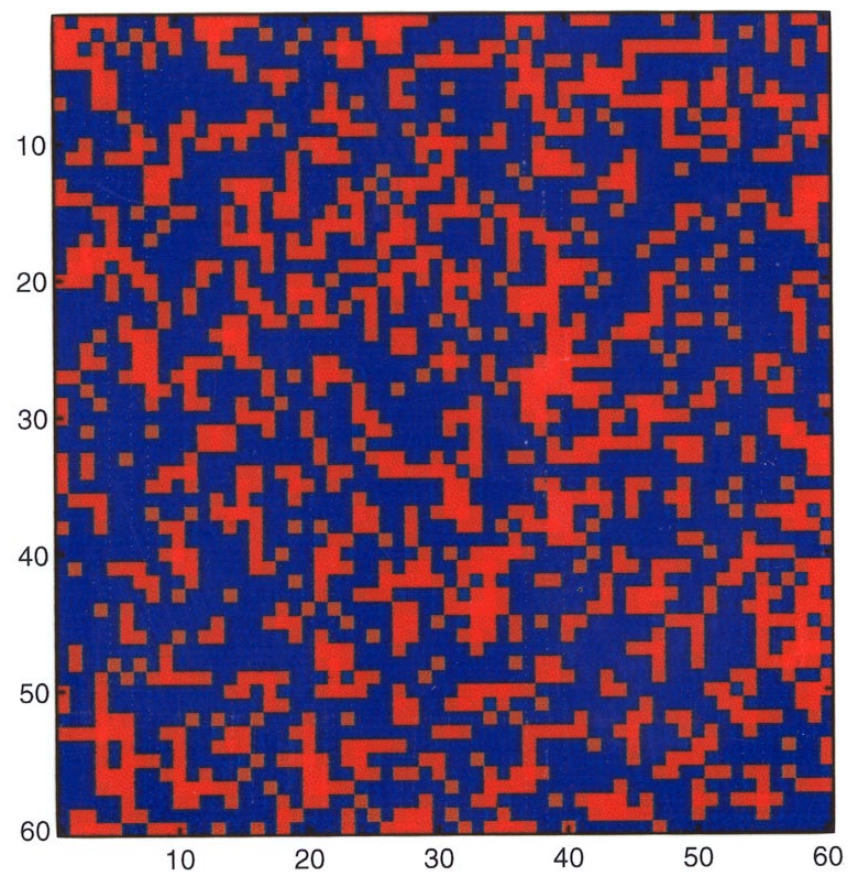

(c)

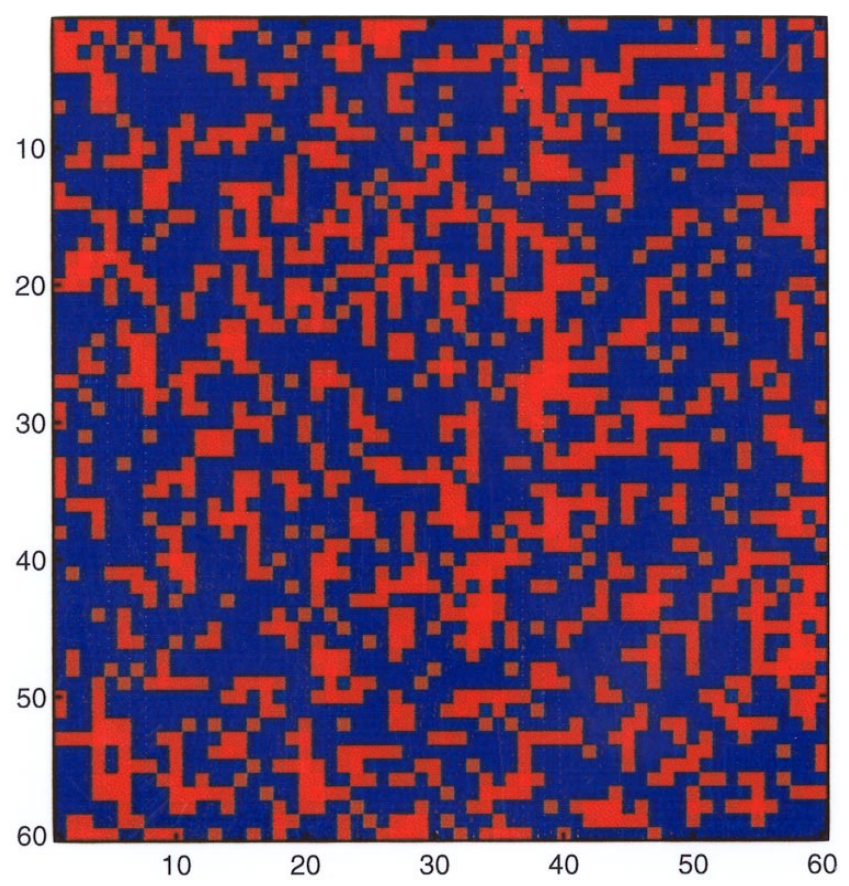

(e)

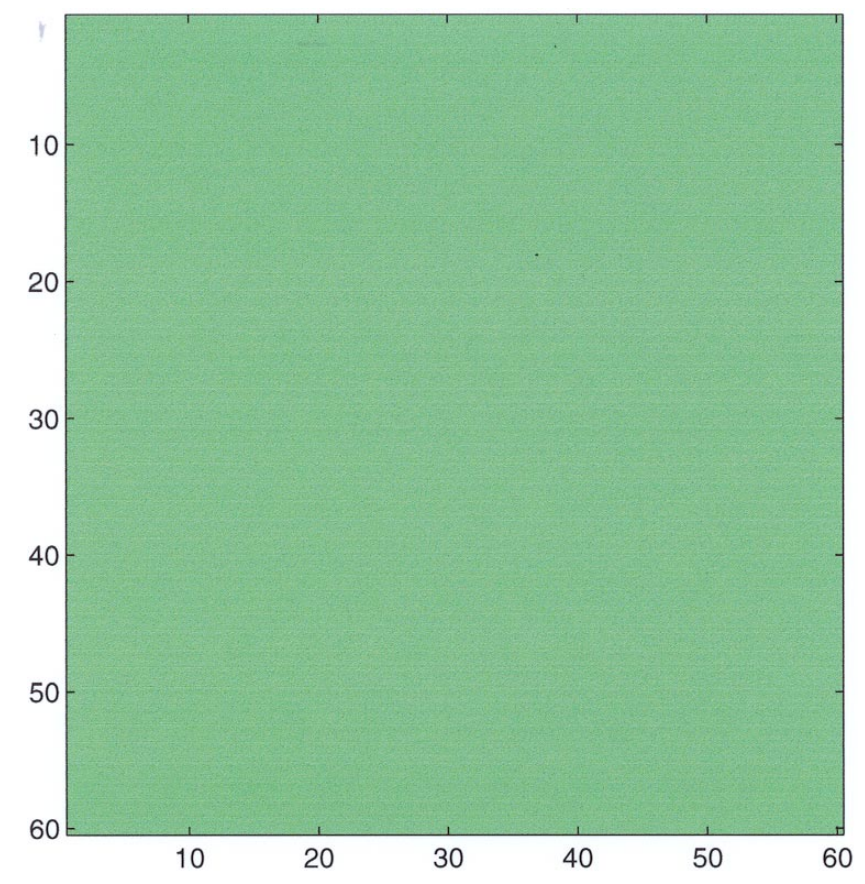

(d)

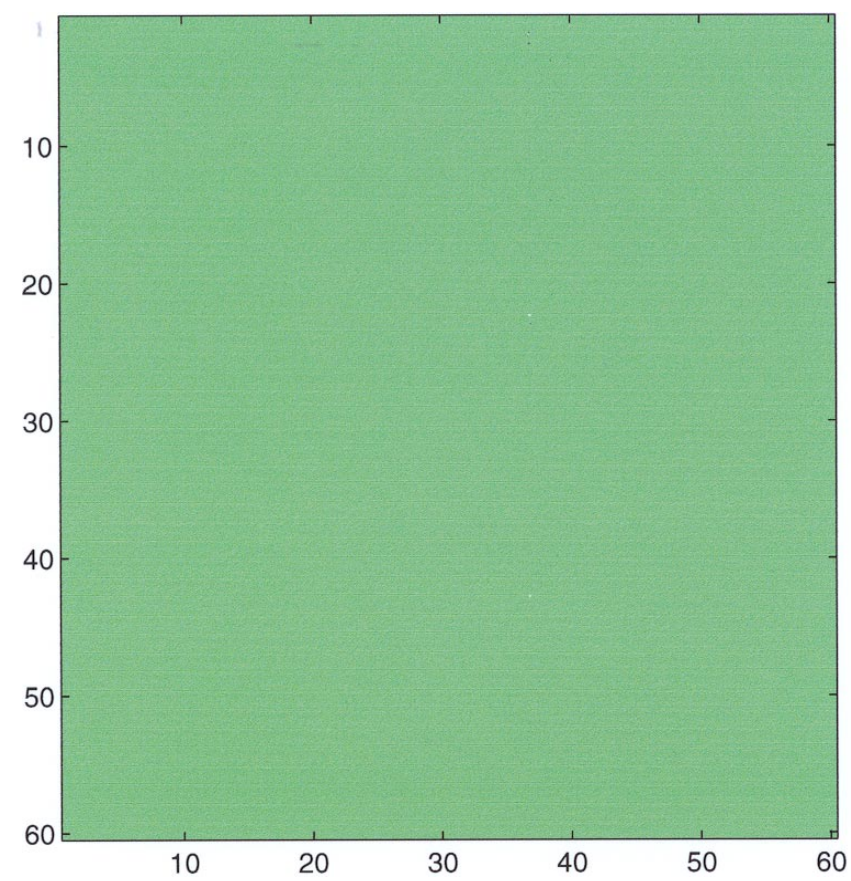

(f)

Fig. 23. (Continued) 


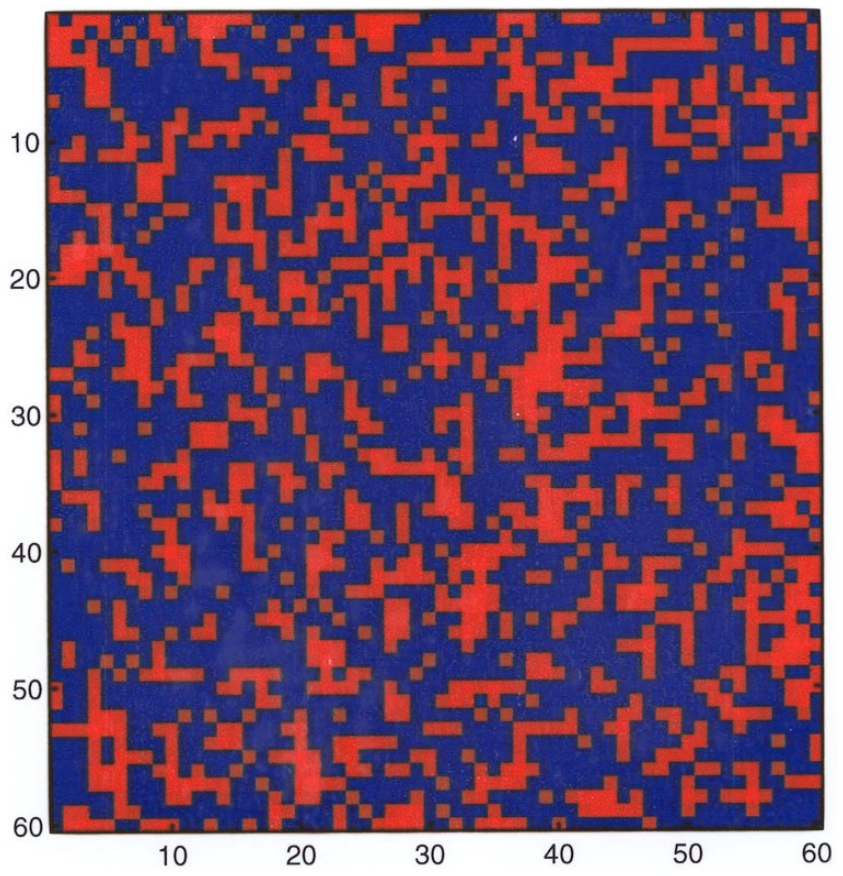

(g)

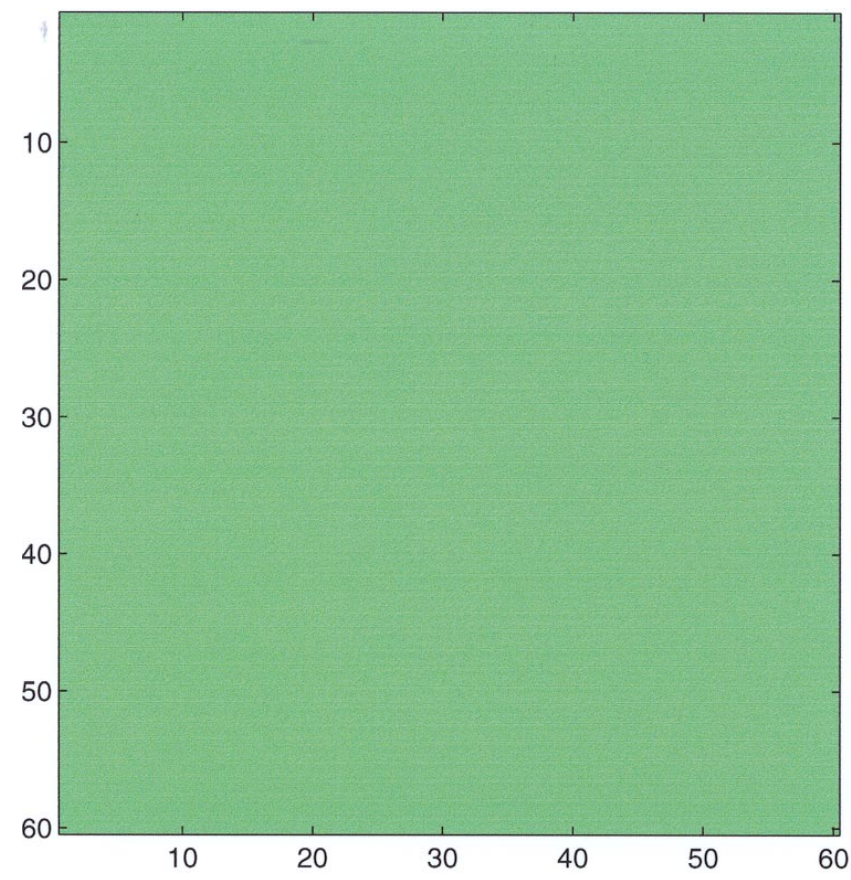

(h)

Fig. 23. (Continued)

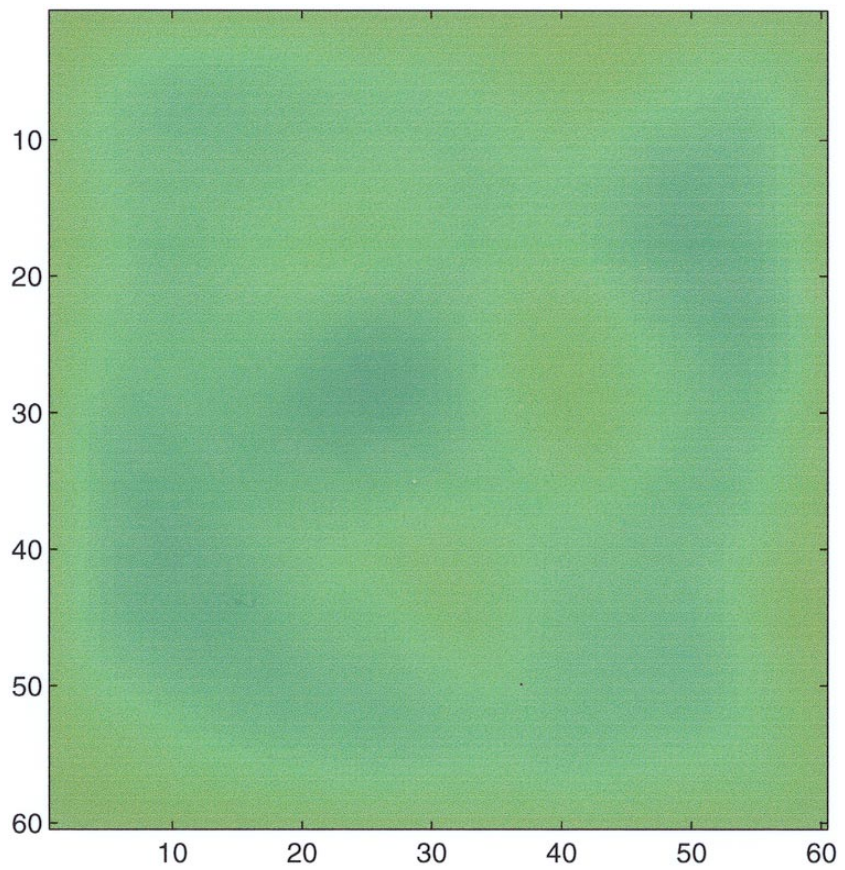

(a)

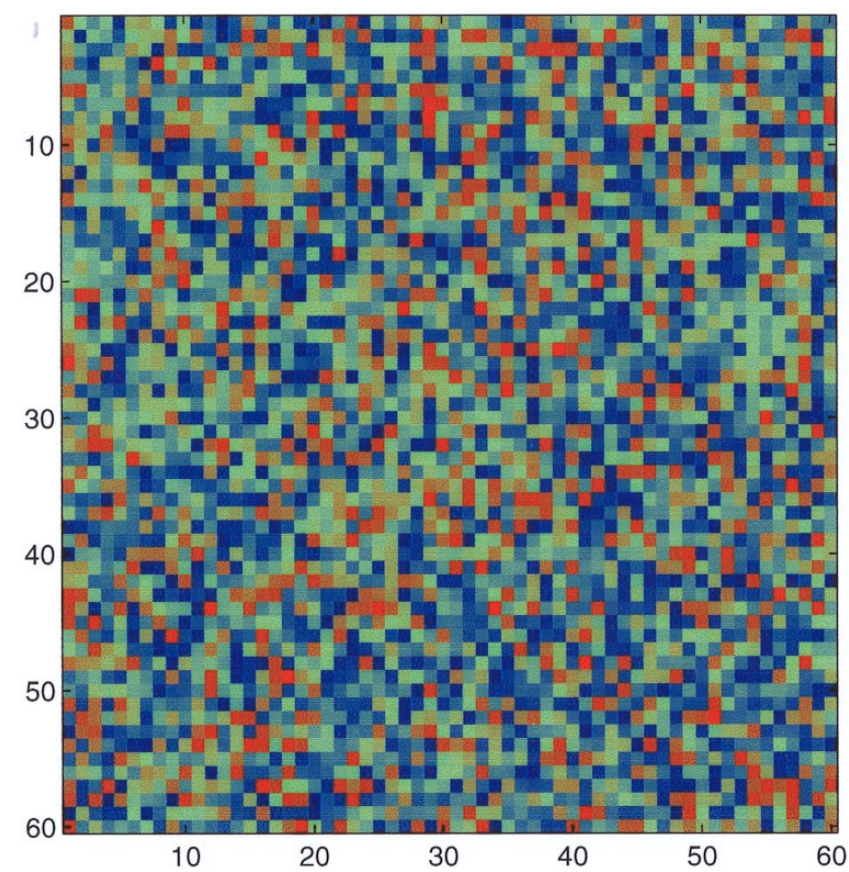

(b)

Fig. 24. Simulation results of Chua's circuit CNN with parameter $\alpha=-0.1, \beta=14, \gamma=0, a=-1, c=2, D_{1}=100$ and $D_{2}=100$. (a) $V_{1}(0.1)$. (b) $V_{2}(0.1)$. (c) $V_{1}(0.2)$. (d) $V_{2}(0.2)$. (e) $V_{1}(1)$. (f) $V_{2}(1)$. (g) $V_{1}(2)$. (h) $V_{2}(2)$. (i) $V_{1}(10)$. (j) $V_{2}(10)$. (k) $V_{1}(20)$. (l) $V_{2}(20)$. 


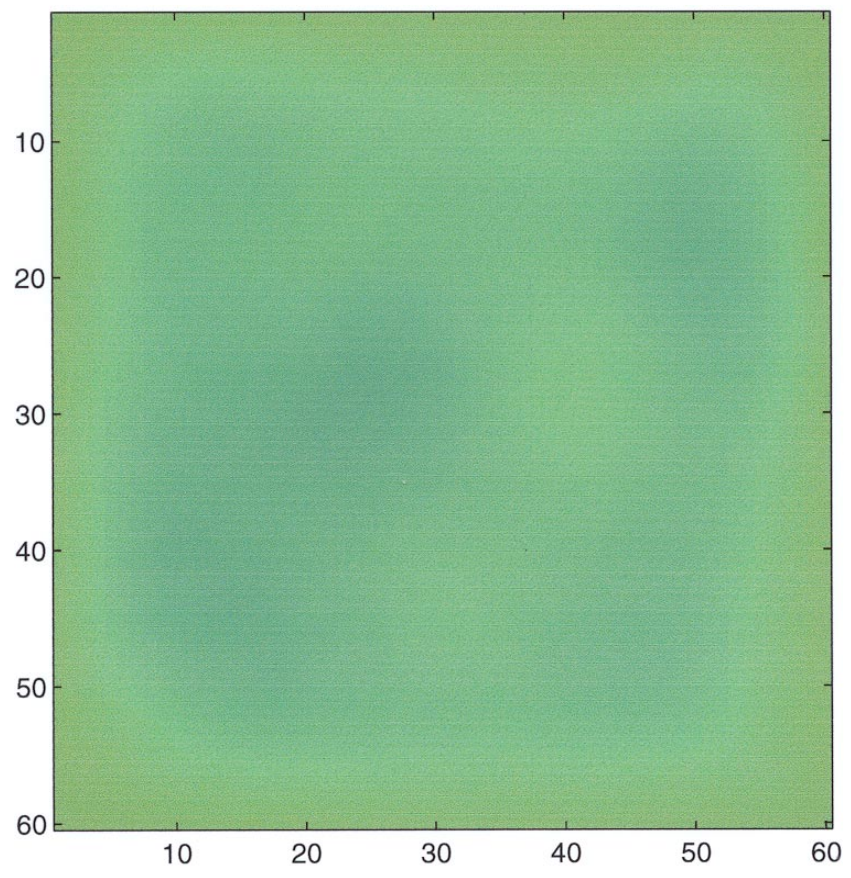

(c)

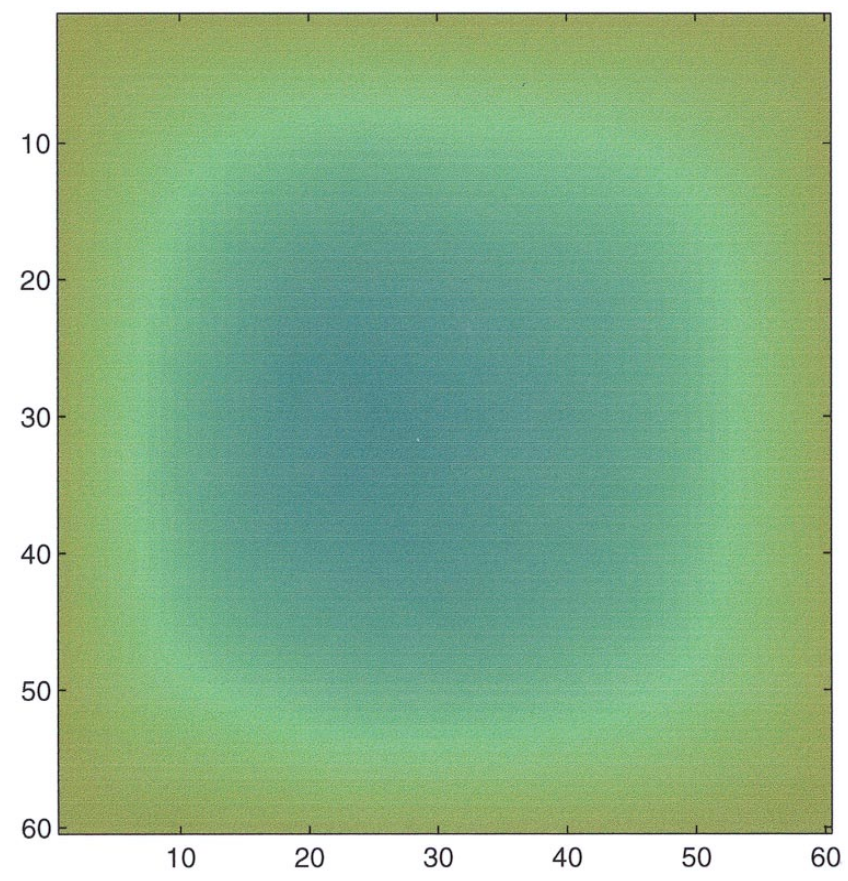

(e)

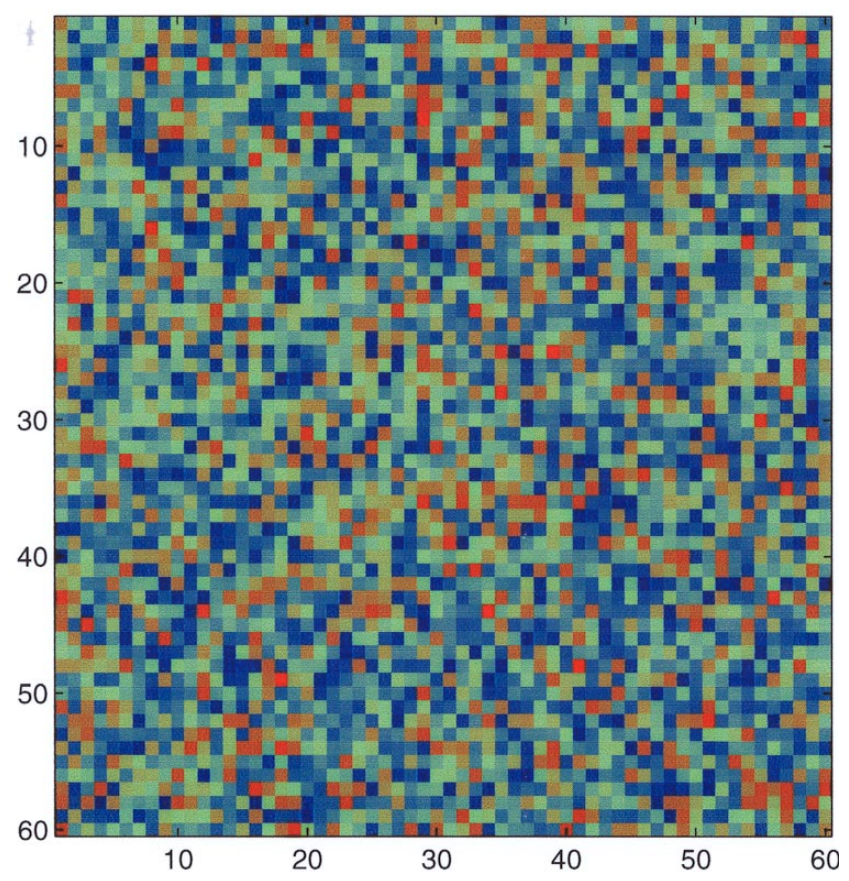

(d)

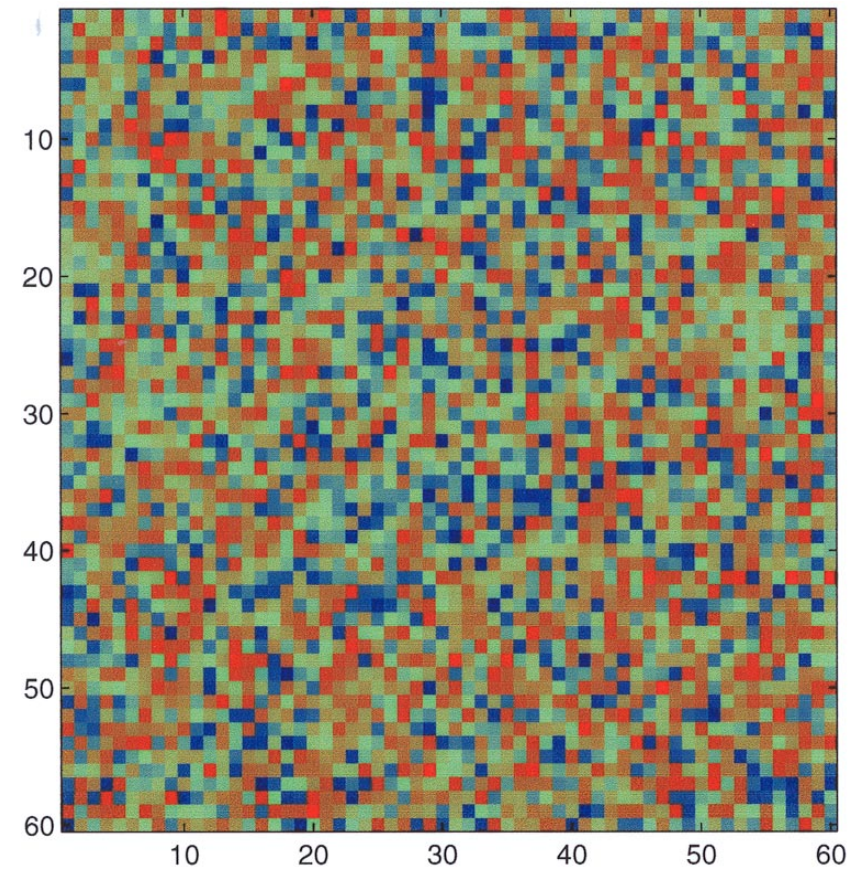

(f)

Fig. 24. (Continued) 


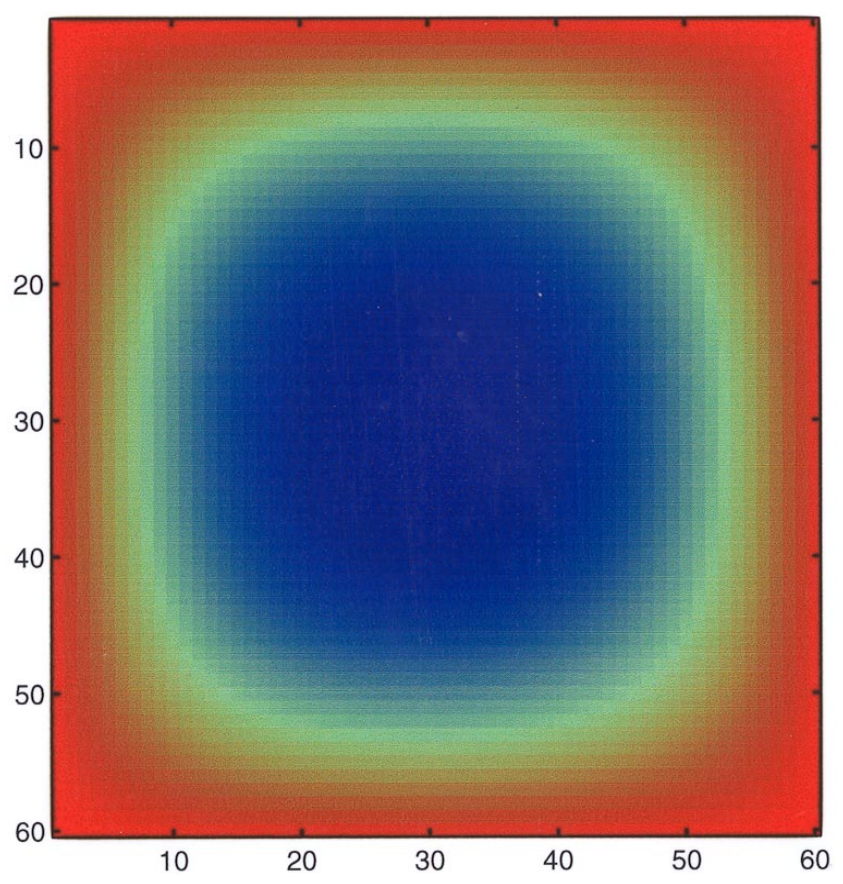

(g)

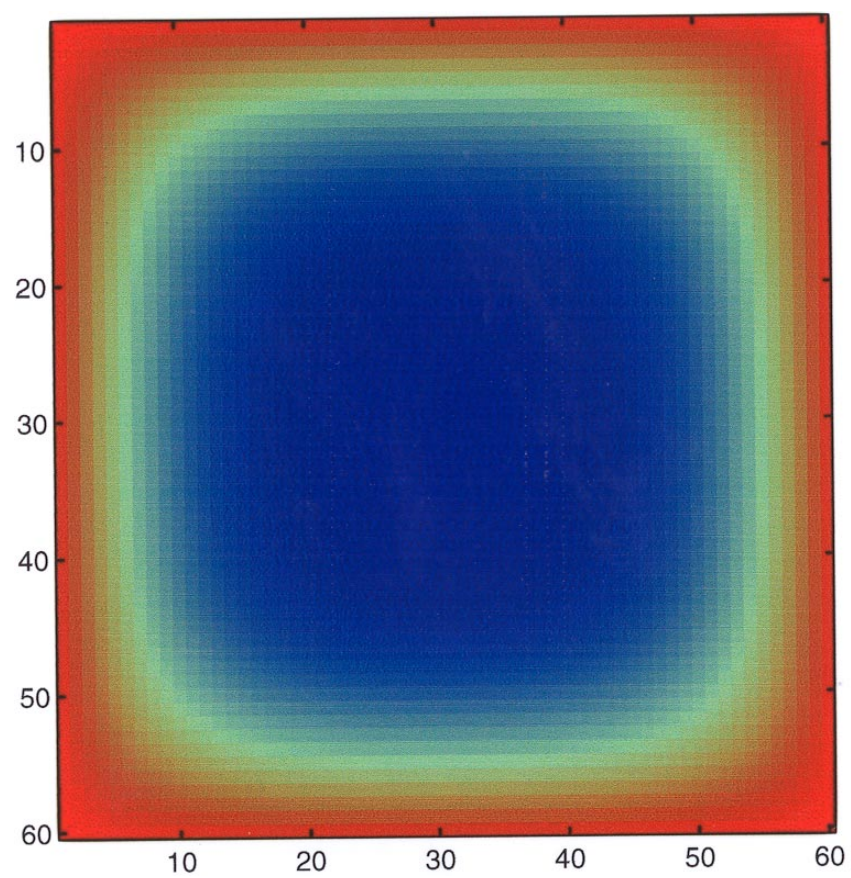

(i)

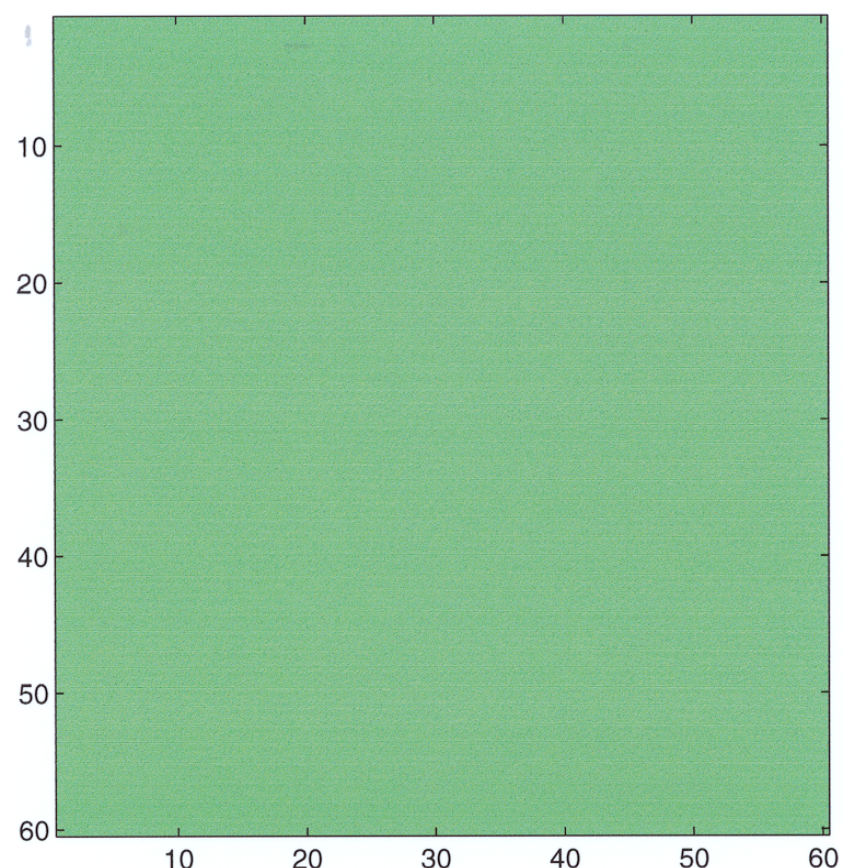

(h)

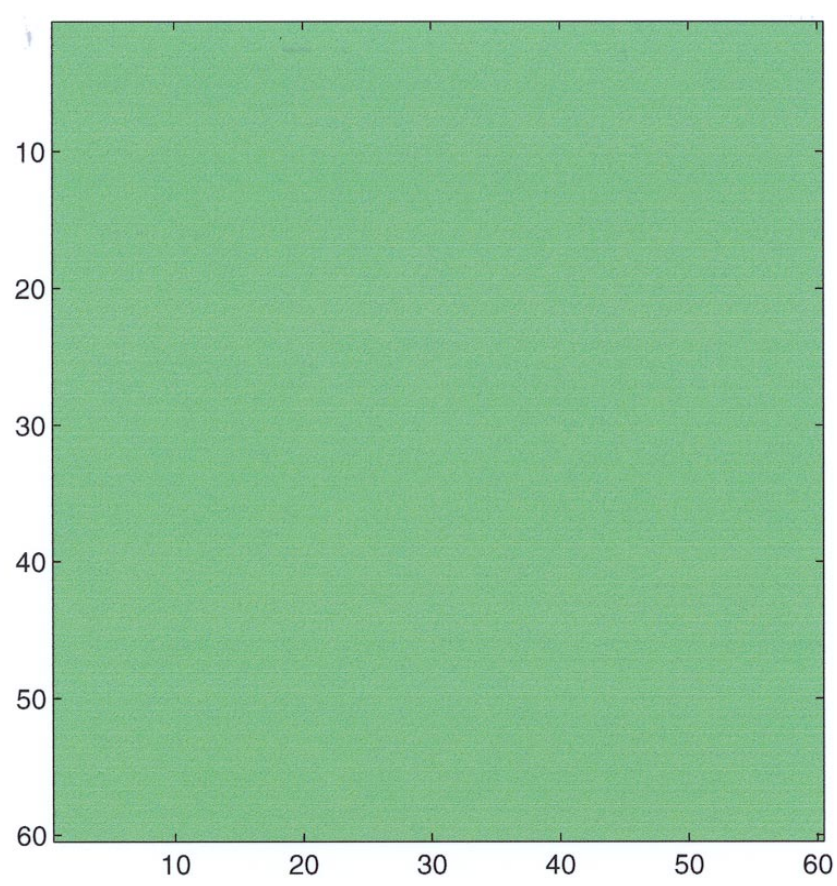

(j)

Fig. 24. (Continued) 


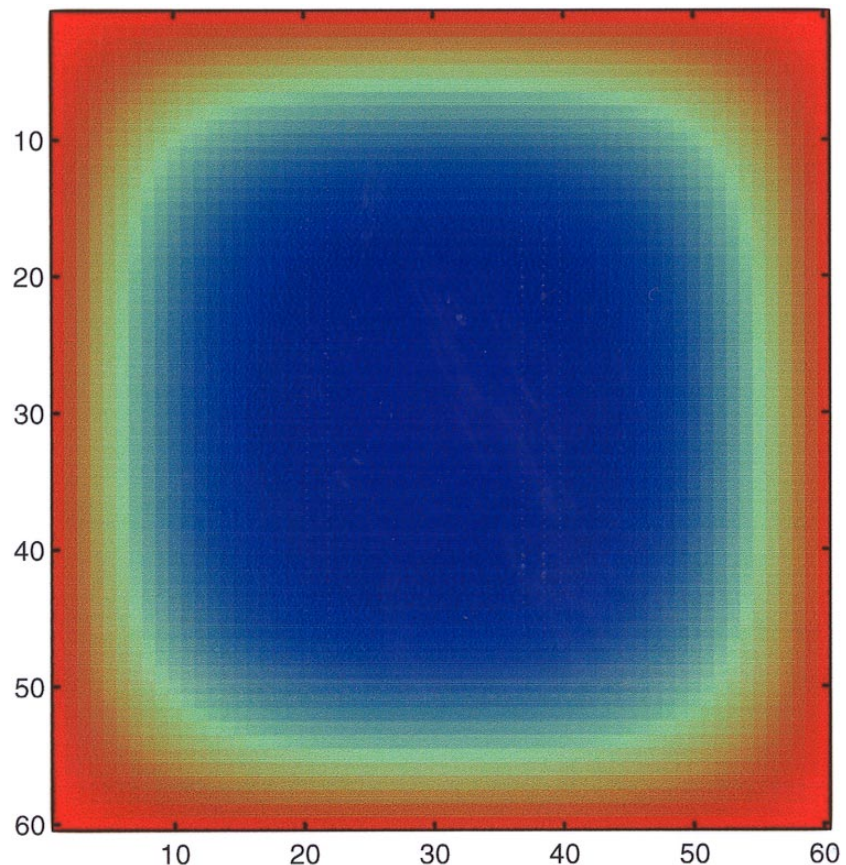

$(\mathrm{k})$

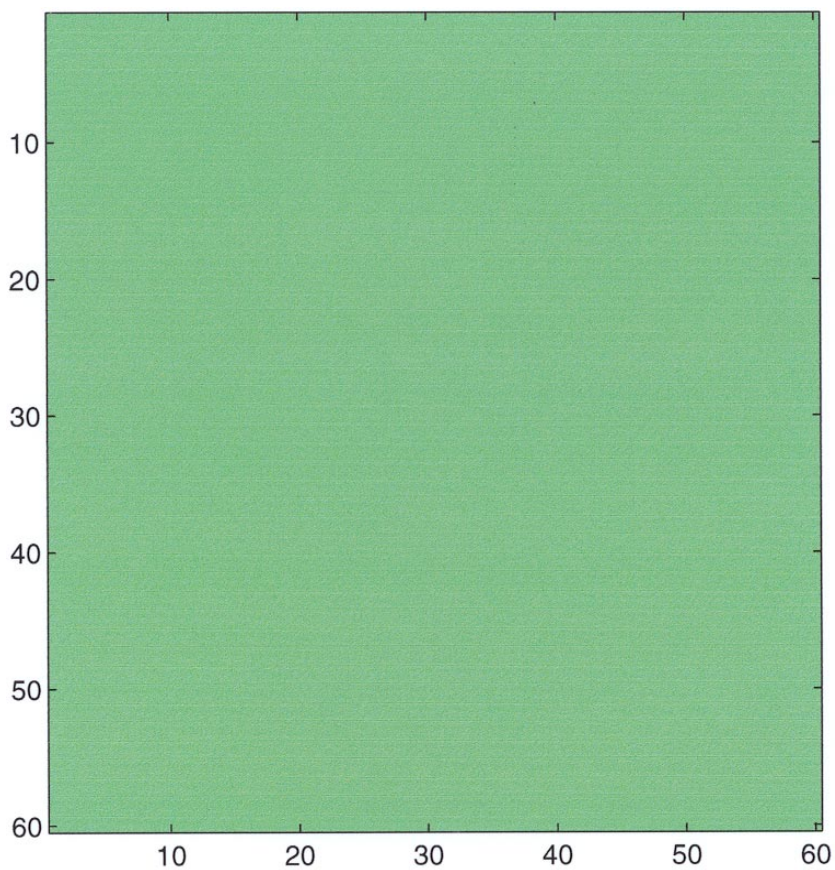

(l)

Fig. 24. (Continued)

with a smooth transient boundary. Observe that $V_{2}(t)$ converges to a homogeneous pattern.

\section{Remarks}

If the coupling is too weak, we observe a "randomlike" static pattern from a pseudo-random initial condition. If the coupling is not too weak, we observe some transient patch patterns. If the coupling is sufficiently strong we observe the CNN converges to a homogeneous pattern rapidly. If we increase the coupling further, we observe some kind of standing target waves. However, if the coupling becomes too strong (for example $D_{1}=D_{2}=1000$ ), we observe unstable patterns. These results are consistent with the behaviors predicted from the parameter plane depicted in Fig 19.

\section{Concluding Remarks}

In this paper we have presented closed from analytical results on locally passive, locally active and edge-of-chaos parameter regions for continuoustime CNNs. Our results shed some light on the connections between pattern formation in a CNN and the shapes of the local active and local pas- sive regions in CNN cell parameter and coupling current spaces. When a CNN cell has more than one port, the criteria for testing local activity and local passivity must be expressed in matrix form. In this paper, we present closed-form conditions for two-port second-order cells, two-port third-order cells and three-port third-order cells. When the order of the cell is greater than 4, in general it will be very difficult to find closed-form expressions for the local passivity criteria because there exist no closed-form expressions for the poles. For one-port cells, since its impedance $Z_{Q}(s)$ can be written as a scalar function of $s$, it is possible to find the edgeof-chaos regions directly from $Z_{Q}(s)$. However, for two-port or three-port cells, the conditions become much more complicated. In many cases it is very difficult, if not impossible, to derive the edge-ofchaos regions analytically from the impedance matrix. To overcome this problem, we devise a method which combines the information obtained from both the impedance and the admittance matrices. We have also verified the classic circuit-theoretic result cited in [Chua, 1998] and [Chua, 1999] that "the local passive regions obtained from the impedance and the admittance matrices are equivalent." Since this fundamental result is not obvious intuitively, 
we have provided self-contained proofs for the oneport first-order, one-port second-order and two-port second-order cases.

\section{Acknowledgments}

This work is supported by the DOD Office of Naval Research (ONR) grant N000-14-98-1-0594.

\section{References}

Brayton, R. K., Chua, L. O., Rhodes, J. D. \& Spence, R. [1978] Nonlinear Network Theory - An Introduction (Georgi, St. Saphorin).

Chua, L. O. [1998] CNN: A Vision of Complexity (World Scientific, Singapore).

Chua, L. O. [1999] "Passivity and complexity," IEEE
Trans. Circuits Syst. I: Fundamental Th. Appl. 46(1), $71-82$.

Dogaru, R. \& Chua, L. O.[1998a] "Edge of chaos and local activity domain of FitzHugh-Nagumo equation," Int. J. Bifurcation and Chaos 8(2), 211-257.

Dogaru, R. \& Chua, L. O.[1998b] "Edge of chaos and local activity domain of the Brusselator CNN," Int. J. Bifurcation and Chaos 8(6), 1107-1130.

Dogaru, R. \& Chua, L. O.[1998c] "Edge of chaos and local activity domain of the Gierer-Meinhardt CNN," Int. J. Bifurcation and Chaos 8(12), 2321-2340.

Franklin, J. N. [1968] Matrix Theory, Prentice-Hall Series in Applied Mathematics (Prentice-Hall, Englewood Cliffs, NJ).

Min, L. Q., Crounse, K. R. \& Chua, L. O. [2000] "Analytical criteria for local activity and applications to the Oregonator CNN," Int. J. Bifurcation and Chaos 10(1), 25-71. 